\title{
Aktualisierte S3-Leitlinie Colitis ulcerosa der Deutschen Gesellschaft für Gastroenterologie, Verdauungs- und Stoffwechselkrankheiten (DGVS)
}

\author{
AWMF-Register-Nr. 021/009
Updated S3-Guideline Colitis ulcerosa. German Society for Digestive and Metabolic Diseases (DGVS)

AWMF Registry 021/009

\begin{abstract}
Authors
Torsten Kucharzik ${ }^{*}$, Axel U. Dignass ${ }^{2 *}$, Raja Atreya ${ }^{3}$, Bernd Bokemeyer ${ }^{4}$, Philip Esters², Klaus Herrlinger ${ }^{5}$, Klaus Kannengießer ${ }^{1}$, Peter Kienle ${ }^{6}$, Jost Langhorst ${ }^{7}$, Andreas Lügering ${ }^{8}$, Stefan Schreiber ${ }^{9}$, Andreas Stallmach ${ }^{10}$, Jürgen Stein ${ }^{11}$, Andreas Sturm ${ }^{12}$, Niels Teich ${ }^{13}$, Britta Siegmund ${ }^{14}$

Collaborators:

T. Andus, F. Autschbach, O. Bachmann, G. Baretton, D. C. Baumgart, D. Bettenworth, M. Bläker, S. Buderus, J. Büning, R. Ehehalt, K. Fellermann, S. Fichtner-Feigl, M. Götz, C. Gross, F. Hartmann, P. Hartmann, S. In der Smitten, W. Häuser, U. Helwig, B. Kaltz, I. Kanbach, K. M. Keller, J. Klaus, S. Koletzko, A. Kroesen, W. Kruis, T. Kühbacher, L. Leifeld, C. Maaser, H. Matthes, G. Moog, J. Ockenga, A. Pace, M. Reinshagen, E. Rijcken, G. Rogler, E. Stange, C. Veltkamp, J. Zemke
\end{abstract}

Institute

1 Klinik für Allgemeine Innere Medizin und Gastroenterologie, Klinikum Lüneburg, Lüneburg, Deutschland

2 Medizinische Klinik I, Agaplesion Markus Krankenhaus, Frankfurt am Main, Deutschland

3 Medizinische Klinik 1, Universitätsklinikum Erlangen, Deutschland

4 Gastroenterologische Gemeinschaftspraxis Minden, Deutschland

5 Innere Medizin I, Asklepios Klinik Nord Hamburg, Deutschland

6 Allgemein- und Viszeralchirurgie, Theresienkrankenhaus und Sankt Hedwig-Klinik GmbH, Mannheim, Deutschland

7 Innere Medizin V, Naturheilkunde und Integrative Medizin, Kliniken Essen-Mitte, University of Duisburg-Essen, Deutschland

8 Medizinisches Versorgungszentrum Portal 10, Münster, Deutschland

9 Klinik für Innere Medizin I, Kiel, Deutschland

10 Gastroenterologie, Hepatologie und Infektiologie, Friedrich Schiller Universität, Jena, Deutschland

11 Interdisziplinäres Crohn und Colitis Centrum Rhein-Main, Frankfurt/Main, Deutschland

12 Klinik für Innere Medizin mit Schwerpunkt Gastroenterologie, DRK Kliniken Berlin Westend, Berlin, Deutschland

Beide Autoren haben in gleicher Weise zur Erstellung des Manuskripts beigetragen.
13 Internistische Gemeinschaftspraxis für Verdauungs- und Stoffwechselkrankheiten, Leipzig, Deutschland

14 Medizinische Klinik I, Charité Universitätsmedizin Berlin, Campus Benjamin Franklin, Berlin, Deutschland

Schlüsselwörter

Colitis ulcerosa, Chronisch-entzündliche Darmerkrankung, Diagnose, Therapie, Remission, Remissionserhaltung

Key words ulcerative colitis, IBD, diagnosis, treatment, remission, maintenance

eingereicht 04.06 .2018

akzeptiert 01.07.2018

Bibliografie

DOI https://doi.org/10.1055/a-0651-8174

Online-Publikation: 11.9.2018

Z Gastroenterol 2018; 56: 1087-1169

(c) Georg Thieme Verlag KG, Stuttgart · New York

ISSN 0044-2771

Korrespondenzadresse

Prof. Dr. med. Torsten Kucharzik

Klinik für Allgemeine Innere Medizin und Gastroenterologie, Städtisches Klinikum Lüneburg, Bögelstr. 1, 21339 Lüneburg torsten.kucharzik@klinikum-lueneburg.de

Prof. Dr. med. Axel Dignass

Medizinische Klinik I, Agaplesion Markus Krankenhaus,

Wilhelm-Epstein-Str. 4, 60431 Frankfurt

axel.dignass@fdk.info 


\section{ZUSAMMENFASSUNG}

Die neue S3-Leitlinie Colitis stellt aktuelle und evidenzbasierte Empfehlungen zur Behandlung der Colitis ulcerosa zur Verfügung. Sie ersetzt damit die Vorläuferversion von 2011. Neben den neuesten Erkenntnissen zu Diagnostik und Therapie werden insbesondere infektiologische Probleme, chirurgische und Ernährungsmaßnahmen aufgegriffen. Unter der Federführung der DGVS wurde die Leitlinie gemeinsam mit 10 weiteren Fachgesellschaften und Patientenvertretern erarbeitet mit dem Ziel, eine optimale interdisziplinäre Versorgung der Patienten zu gewährleisten.

\section{ABSTRACT}

This guideline provides evidence-based key recommendations for diagnosis and treatment of ulcerative colitis and upgrades the 2011 version. The guideline was developed by an interdisciplinary team of gastroenterologists, surgeons, pathologists, nutrition experts, and patient support groups under the auspice of the German Society for Gastroenterology and Metabolic Diseases. The guideline used structural S3 consensus-based methodology and includes statements on clinical practice, prevention, infectiological problems, surgery and nutrition.

\begin{tabular}{|c|c|c|}
\hline \multicolumn{2}{|c|}{ Inhaltsverzeichnis } & \multirow{2}{*}{$\begin{array}{l}\text { Seite } \\
1088\end{array}$} \\
\hline 1. & Informationen zu dieser Leitlinie & \\
\hline & Herausgeber & 1088 \\
\hline & Methodologische Exaktheit & 1089 \\
\hline & Externe Begutachtung und Verabschiedung & 1092 \\
\hline & Verbreitung und Implementierung & 1092 \\
\hline & Redaktioneller Hinweis & 1092 \\
\hline & Besonderer Hinweis & 1093 \\
\hline \multirow[t]{4}{*}{2.} & Diagnostik & 1093 \\
\hline & Klassifikation & 1093 \\
\hline & Krankengeschichte & 1093 \\
\hline & Diagnosestellung & 1095 \\
\hline \multirow[t]{8}{*}{3.} & $\begin{array}{l}\text { Behandlung der aktiven Erkrankung } \\
\text { und remissionserhaltende Therapie }\end{array}$ & 1105 \\
\hline & Allgemeine Therapieziele & 1105 \\
\hline & Therapie der unkomplizierten Colitis ulcerosa & 1106 \\
\hline & $\begin{array}{l}\text { Remissionserhaltung bei primär unkomplizierter } \\
\text { Colitis ulcerosa }\end{array}$ & 1108 \\
\hline & $\begin{array}{l}\text { Komplizierte Verlaufsformen der Colitis ulcerosa/ } \\
\text { Schwere Colitis ulcerosa }\end{array}$ & 1111 \\
\hline & Colitis ulcerosa mit steroidabhängigem Verlauf & 1116 \\
\hline & Anwendung von Biosimilars bei Colitis ulcerosa & 1119 \\
\hline & $\begin{array}{l}\text { Remissionserhaltung bei kompliziertem Verlauf } \\
\text { einer Colitis ulcerosa }\end{array}$ & 1120 \\
\hline 4. & Infektiologische Probleme & 1124 \\
\hline 5. & Chirurgie & 1131 \\
\hline 5.1 & Operative Verfahren & 1131 \\
\hline 5.2 & Pouchitis & 1137 \\
\hline 6. & Ernährung und komplementäre Verfahren & 1140 \\
\hline 6.1 & $\begin{array}{l}\text { Ernährung in der Ätiologie und Prävention } \\
\text { der Colitis ulcerosa }\end{array}$ & 1140 \\
\hline 6.2 & Komplementäre Therapieverfahren & 1143 \\
\hline \multicolumn{2}{|c|}{ Abkürzungen } & 1148 \\
\hline \multicolumn{2}{|c|}{ Literatur } & 1149 \\
\hline
\end{tabular}

\section{Informationen zu dieser Leitlinie}

\section{Herausgeber}

\section{Federführende Fachgesellschaft}

Deutsche Gesellschaft für Gastroenterologie, Verdauungs- und Stoffwechselkrankrankheiten (DGVS)

\section{Geltungsbereich und Zweck}

In Deutschland sind etwa 150000 Einwohner an einer Colitis ulcerosa erkrankt. Für die meisten Patienten beginnt die Erkrankung während der Schulzeit oder der Berufsausbildung und dauert während des gesamten Lebens an. Neben dem persönlichen Leiden verursacht die Erkrankung beträchtliche Kosten für die Gesellschaft. Viele Patienten erhalten jedoch nach wie vor keine adäquate Therapie. Die Aktualisierung der zuletzt 2011 aktualisierten Leitlinie wird von den beteiligten Fachgesellschaften daher als besonders wichtig erachtet.

\section{Zielorientierung der Leitlinie}

Ziel der Leitlinie soll es sein, in der hausärztlichen, internistischen, chirurgischen, pädiatrischen und gastroenterologischen Praxis einfach anwendbar zu sein. Die Behandlung besonders schwerer oder komplizierter Fälle, wie sie in Spezialambulanzen und spezialisierten Praxen erfolgt, kann durch diese Leitlinie nicht vollständig abgebildet werden.

Die Themen „Extraintestinale Manifestationen“ und „CEDassoziierte Erkrankungen“ sowie „Schmerzen“ wurden in der letzten Morbus-Crohn-Leitlinie von 2014 abgehandelt und sollten daher in der Colitis-ulcerosa-Leitlinie nicht erneut aufgegriffen werden. „Infektiologische Probleme“ sowie das Thema „Ernährung“ wurden in der letzten Morbus-Crohn-Leitlinie nicht fokussiert und sollten daher in dieser Leitlinie gezielt aufgearbeitet werden.

Patientenzielgruppe sind Patienten jeden Alters mit Colitis ulcerosa.

\section{Versorgungsbereich}

Ambulant und stationär, hausärztlich, pädiatrisch, internistisch, chirurgisch und gastroenterologisch. 
- Tab. 1 Steuergruppe.

\begin{tabular}{|l|l|l|}
\hline Name & Ort & Zuständigkeit \\
\hline B. Bokemeyer & Minden & Vertreter der Niedergelassenen Gastroenterologen, Kompetenznetz KN-CED \\
\hline A. Dignass & Frankfurt & Koordinator, ALGK, DGVS \\
\hline B. Kaltz & Berlin & DCCV \\
\hline T. Kucharzik & Lüneburg & Koordinator, ALGK, ECCO \\
\hline S. Schreiber & Kiel & Kompetenznetz KN-CED, universitäre Gastroenterologie \\
\hline B. Siegmund & Berlin & ECCO, universitäre Gastroenterologie, DGVS \\
\hline & & \\
\hline
\end{tabular}

\section{Anwenderzielgruppe/Adressaten}

Die Leitlinie richtet sich an alle an der Diagnostik und Therapie beteiligten Berufsgruppen (Allgemeinmediziner, Internisten, Kinder- und Jugendmediziner, Chirurgen, Gastroenterologen, Pathologen, Fachassistenz CED) sowie Betroffene und Angehörige und Leistungserbringer (Krankenkassen, Rentenversicherungsträger).

\section{Zusammensetzung der Leitliniengruppe:}

\section{Beteiligung von Interessengruppen}

Die Leitung der Leitlinienüberarbeitung erfolgte durch zwei Hauptkoordinatoren (Axel Dignass, Frankfurt, und Torsten Kucharzik, Lüneburg) in enger Abstimmung mit einer Steuergruppe $(\triangleright$ Tab. 1).

Neben diesen Leitungsgremien wurden fünf Arbeitsgruppen (AGs) gebildet, die jeweils von zwei Leitern geleitet wurden ( $\vee$ Tab. 2). Aufgrund des Umfangs der zu bearbeitenden Themen wurde die AG Diagnostik von drei AG-Leitern geleitet. In den AGs wurden universitäre und nichtuniversitäre Ärzte, Klinikärzte und niedergelassene Ärzte in einem ausgewogenen Verhältnis eingesetzt. In den AGs haben neben Gastroenterologen und Chirurgen Pädiater, Pathologen, Komplementärmediziner, Ernährungsmediziner, Fachassistenzen CED (FACED) und Patienten (DCCV) mitgearbeitet.

Aus jeder AG haben alle Mitglieder an der Onlinebefragung und fast alle Mitglieder an der Konsensuskonferenz teilgenommen.

Repräsentativität der Leitliniengruppe:

Beteiligte Fachgesellschaften

- DGVS (Deutsche Gesellschaft für Gastroenterologie, Verdauungs- und Stoffwechselkrankheiten)

- DGAV (Deutsche Gesellschaft für Allgemein- und Viszeralchirurgie)

- DGCH (Deutsche Gesellschaft für Chirurgie)

- GPGE (Gesellschaft für pädiatrische Gastroenterologie und Ernährungsmedizin)

- KN-CED (Kompetenznetz Darmerkrankungen)

- DCCV e. V. (Deutsche Morbus Crohn/Colitis ulcerosa Vereinigung)

- FACED (Fachangestellte für chronisch entzündliche Darmerkrankungen)

- DGEM (Deutsche Gesellschaft für Ernährungsmedizin)
- DGP (Deutsche Gesellschaft für Pathologie)

- DGK (Deutsche Gesellschaft für Koloproktologie)

Die Deutsche Gesellschaft für Allgemeinmedizin und Familienmedizin (DEGAM) wurde zur Mitarbeit an der Leitlinie eingeladen, konnte das Leitlinienvorhaben aber aufgrund personeller Engpässe nicht unterstützen. Auch die Deutsche Gesellschaft für Innere Medizin e. V. DGIM) wurde zur Mitarbeit an der Leitlinie eingeladen, hat sich aber nicht beteiligen können.

Repräsentativität der Leitliniengruppe:

Beteiligung von Patienten

Direkte Mitarbeit von mehreren Vertretern der Deutschen Morbus Crohn/Colitis ulcerosa Vereinigung (DCCV) e. V.

\section{Methodologische Exaktheit}

\section{Recherche, Auswahl und Bewertung wissenschaftlicher Belege (Evidenzbasierung)}

Vorgängerversionen dieser Leitlinie sind die S3-Leitlinien Colitis ulcerosa von 2011 und 2008. Aufgrund neuer methodischer Anforderungen wurde vor Beginn der Überarbeitung die anzuwendende Methodik am 05.07.2016 im Rahmen einer Telefonkonferenz innerhalb der Steuergruppe diskutiert und anschließend konsentiert.

Die Suchstrategie der letzten Colitis-Leitlinie wurde von den Koordinatoren zusammen mit den AG-Leitern überarbeitet. Mit der systematischen Literaturrecherche dieser Aktualisierung wurde die clinical guideline services usergroup (CGS) beauftragt; die Literaturrecherche wurde durchgeführt durch Frau Maria Kallenbach. Zunächst wurde eine systematische Suche nach Leitlinien und eine Bewertung nach dem Deutschen Instrument zur methodischen Leitlinienbewertung (DELBI) durchgeführt und eine Leitliniensynopse erstellt.

Für Themengebiete von besonderer klinischer Wichtigkeit, besonderer Strittigkeit oder besonders häufiger fehlerhafter Anwendung wurden von den AG-Leitern Schlüsselfragen definiert, eine systematische Literaturrecherche wurde de novo durchgeführt und Evidenztabellen wurden erstellt. Die Literatur konnte bis zum Zeitpunkt der Konsensuskonferenz ergänzt werden durch Arbeiten, die den AG-Mitgliedern bekannt waren, die aber nicht durch die systematische Suche erfasst wurden. Empfehlungen, 
- Tab.2 Mitglieder der Leitliniengruppe.

\begin{tabular}{|c|c|c|}
\hline \multirow[t]{2}{*}{ AG 1: Diagnostik } & Leiter & $\begin{array}{l}\text { R. Atreya, Erlangen (DGVS) } \\
\text { B. Bokemeyer, Minden (KN-CED, DGVS) } \\
\text { K. Herrlinger, Hamburg (DGVS) }\end{array}$ \\
\hline & KK-Teilnehmer & $\begin{array}{l}\text { D. Bettenworth, Münster (DGVS) } \\
\text { M. Götz, Tübingen (DGVS) } \\
\text { U. Helwig, Oldenburg (DGVS) } \\
\text { L. Leifeld, Hildesheim (DGVS) } \\
\text { G. Moog, Kassel (DGVS) } \\
\text { E. Rijcken, Münster (DGAV/DGK) } \\
\text { F. Autschbach, Heilbronn (DGP) } \\
\text { G. Baretton, Dresden (DGP) } \\
\text { I. Kanbach, Berlin (DCCV) } \\
\text { S. Buderus, Bonn (GPGE) } \\
\text { P. Hartmann, Minden (FACED) }\end{array}$ \\
\hline \multirow[t]{2}{*}{ AG 2: Schub } & Leiter & $\begin{array}{l}\text { T. Kucharzik, Lüneburg (DGVS, KN-CED) } \\
\text { B. Siegmund, Berlin (DGVS, KN-CED) }{ }^{1}\end{array}$ \\
\hline & KK-Teilnehmer & $\begin{array}{l}\text { J. Büning, Lübeck (DGVS) } \\
\text { R. Ehehalt, Heidelberg (DGVS) } \\
\text { W. Häuser, Saarbrücken (DGVS) } \\
\text { F. Hartmann, Frankfurt (DGVS) } \\
\text { K. Kannengießer, Lüneburg (DGVS) } \\
\text { K.-M. Keller, Wiesbaden (GPGE) } \\
\text { A. Lügering, Münster (DGVS) } \\
\text { S. In der Smitten, Berlin (DCCV) } \\
\text { J. Zemke, Herne (FACED) }\end{array}$ \\
\hline \multirow[t]{2}{*}{ AG 3: Remissionserhaltung } & Leiter & $\begin{array}{l}\text { A. Dignass, Frankfurt (DGVS) } \\
\text { S. Schreiber, Kiel (KN-CED, DGVS) }\end{array}$ \\
\hline & KK-Teilnehmer & $\begin{array}{l}\text { C. Maaser, Lüneburg (DGVS) } \\
\text { G. Rogler, Zürich (DGVS) } \\
\text { S. Koletzko, München (GPGE) } \\
\text { T. Kühbacher, Hamburg (DGVS) } \\
\text { W. Kruis, Köln (DGVS) } \\
\text { P. Esters, Frankfurt (DGVS) }\end{array}$ \\
\hline \multirow[t]{2}{*}{ AG 4: Therapie- und CED-assoziierte Infektionen } & Leiter & $\begin{array}{l}\text { A. Stallmach, Jena (DGVS)1 } \\
\text { N. Teich, Leipzig (DGVS) }\end{array}$ \\
\hline & KK-Teilnehmer & $\begin{array}{l}\text { M. Reinshagen, Braunschweig (DGVS) } \\
\text { T. Andus, Stuttgart (DGVS) } \\
\text { O. Bachmann, Hannover (DGVS) } \\
\text { M. Bläker, Hamburg (DGVS) } \\
\text { C. Veltkamp, Heidelberg (DGVS) }\end{array}$ \\
\hline \multirow[t]{2}{*}{ AG 5: Chirurgie/Pouchitis } & Leiter & $\begin{array}{l}\text { P. Kienle, Heidelberg (DGAV/DGK) } \\
\text { A. Sturm, Berlin (DGVS) }\end{array}$ \\
\hline & KK-Teilnehmer & $\begin{array}{l}\text { S. Fichtner-Feigl, Freiburg (DGAV/DGCH/DGK)ํ․ } \\
\text { K. Fellermann, Lübeck (DGVS) } \\
\text { E. Stange, Stuttgart (DGVS) } \\
\text { A. Kroesen, Köln (DGAV/DGCH/DGK) } \\
\text { A. Pace, Neumünster (DGVS) } \\
\text { B. Kaltz, Berlin (DCCV) }\end{array}$ \\
\hline \multirow[t]{2}{*}{ AG 6: Komplementärmedizin und Ernährung } & Leiter & $\begin{array}{l}\text { J. Langhorst, Essen (DGVS) } \\
\text { J. Stein, Frankfurt (DGVS) }\end{array}$ \\
\hline & KK-Teilnehmer & $\begin{array}{l}\text { H. Matthes, Berlin (DGVS) } \\
\text { D. C. Baumgart, Berlin (DGVS) } \\
\text { J. Ockenga, Bremen (DGEM, DGVS) } \\
\text { J. Klaus, Ulm (DGVS) } \\
\text { C. Gross, Berlin (DCCV) }\end{array}$ \\
\hline Koordinatoren & & $\begin{array}{l}\text { A. Dignass, Frankfurt (DGVS) } \\
\text { T. Kucharzik, Lüneburg (DGVS) }\end{array}$ \\
\hline
\end{tabular}




\begin{tabular}{|c|c|c|c|c|c|}
\hline Question & $\begin{array}{l}\text { Step } 1 \\
\text { (Level 1*) }\end{array}$ & $\begin{array}{l}\text { Step } 2 \\
\text { (Level 2*) }\end{array}$ & $\begin{array}{l}\text { Step } 3 \\
\text { (Level } 3^{*} \text { ) }\end{array}$ & $\begin{array}{l}\text { Step } 4 \\
\text { (Level } 4^{*} \text { ) }\end{array}$ & Step 5 (Level 5) \\
\hline $\begin{array}{l}\text { How common is the } \\
\text { problem? }\end{array}$ & $\begin{array}{l}\text { Local and current random sample } \\
\text { surveys (or censuses) }\end{array}$ & $\begin{array}{l}\text { Systematic review of surveys } \\
\text { that allow matching to local } \\
\text { circumstances** }\end{array}$ & Local non-random sample & Case-series". & $\mathrm{h} / \mathrm{a}$ \\
\hline $\begin{array}{l}\text { Is this diagnostic or } \\
\text { monitoring test } \\
\text { accurate? } \\
\text { (Diagnosis) }\end{array}$ & $\begin{array}{l}\text { Systematic review } \\
\text { of cross sectional studies with } \\
\text { consistently applied reference } \\
\text { standard and blinding }\end{array}$ & $\begin{array}{l}\text { Individual cross sectional } \\
\text { studies with consistently } \\
\text { applied reference standard and } \\
\text { blinding }\end{array}$ & $\begin{array}{l}\text { Non-consecutive studies, or studies without } \\
\text { consistently applied reference standards** }\end{array}$ & $\begin{array}{l}\text { Case-control studies, or } \\
\text { "poor or non-independent } \\
\text { reference standard** }\end{array}$ & $\begin{array}{l}\text { Mechanism-based } \\
\text { reasoning }\end{array}$ \\
\hline $\begin{array}{l}\text { What will happen if } \\
\text { we do not add a } \\
\text { therapy? } \\
\text { (Prognosis) }\end{array}$ & $\begin{array}{l}\text { Systematic review } \\
\text { of inception cohort studies }\end{array}$ & Inception cohort studies & Cohort study or control arm of randomized trial* & $\begin{array}{l}\text { Case-series or case- } \\
\text { control studies, or poor } \\
\text { quality prognostic cohort } \\
\text { study-* }\end{array}$ & $\mathrm{h} / \mathrm{a}$ \\
\hline $\begin{array}{l}\text { Does this } \\
\text { intervention help? } \\
\text { (Treatment Benefits) }\end{array}$ & $\begin{array}{l}\text { Systematic review } \\
\text { of randomized trials or } n \text {-of- } 1 \text { trials }\end{array}$ & $\begin{array}{l}\text { Randomized trial } \\
\text { or observational study with } \\
\text { dramatic effect }\end{array}$ & $\begin{array}{l}\text { Non-randomized controlled cohort/follow-up } \\
\text { study** }\end{array}$ & $\begin{array}{l}\text { Case-series, case-control } \\
\text { studies, or historically } \\
\text { controlled studies*** }\end{array}$ & $\begin{array}{l}\text { Mechanism-based } \\
\text { reasoning }\end{array}$ \\
\hline $\begin{array}{l}\text { What are the } \\
\text { COMMON harms? } \\
\text { (Treatment Harms) }\end{array}$ & $\begin{array}{l}\text { Systematic review of randomized } \\
\text { trials, systematic review } \\
\text { of nested case-control studies, } n \text { - } \\
\text { of-1 trial with the patient you are } \\
\text { raising the question about, or } \\
\text { observational study with dramatic } \\
\text { effect }\end{array}$ & $\begin{array}{l}\text { Individual randomized trial } \\
\text { or (exceptionally) observational } \\
\text { study with dramatic effect }\end{array}$ & $\begin{array}{l}\text { Non-randomized controlled cohort/follow-up } \\
\text { study (post-marketing surveillance) provided } \\
\text { there are sufficient numbers to rule out a } \\
\text { common harm. (For long-term harms the } \\
\text { duration of follow-up must be sufficient.).*. }\end{array}$ & $\begin{array}{l}\text { Case-series, case-control, } \\
\text { or historically controlled } \\
\text { studies** }\end{array}$ & $\begin{array}{l}\text { Mechanism-based } \\
\text { reasoning }\end{array}$ \\
\hline $\begin{array}{l}\text { What are the RARE } \\
\text { harms? } \\
\text { (Treatment Harms) }\end{array}$ & $\begin{array}{l}\text { Systematic review of randomized } \\
\text { trials or } n \text {-of-1 trial }\end{array}$ & \begin{tabular}{|l|} 
Randomized trial \\
or (exceptionally) observational \\
study with dramatic effect
\end{tabular} & & & \\
\hline $\begin{array}{l}\text { Is this (early } \\
\text { detection) test } \\
\text { worthwhile? } \\
\text { (Screening) }\end{array}$ & $\begin{array}{l}\text { Systematic review of randomized } \\
\text { trials }\end{array}$ & Randomized trial & $\begin{array}{l}\text { Non -randomized controlled cohort/follow-up } \\
\text { study** }\end{array}$ & $\begin{array}{l}\text { Case-series, case-control, } \\
\text { or historically controlled } \\
\text { studies** }\end{array}$ & $\begin{array}{l}\text { Mechanism-based } \\
\text { reasoning }\end{array}$ \\
\hline
\end{tabular}

- Abb. 1 CEBM Levels of Evidence 2011.

für die keine neue Evidenz vorlag, wurden unverändert aus der alten Leitlinie übernommen.

Die Literaturbewertung wurde nach der Evidenzklassifizierung des Oxford Centre for Evidence-based Medicine 2011 ( $\triangleright$ Abb. 1) durchgeführt.

Die Details zur Suche und zu Auswahl und Bewertung der Evidenz sind im Leitlinienreport dargestellt.

\section{Formulierung der Empfehlungen und strukturierte Konsensfindung}

Auf der Grundlage der Recherche, Auswahl und Bewertung der Evidenz wurden die Empfehlungen und Hintergrundtexte durch die AGs erarbeitet und zunächst im E-Mail-Umlaufverfahren innerhalb der einzelnen AGs abgestimmt. Bei der Überführung der Evidenzstärke in die Empfehlungsstärke konnte der Empfehlungsgrad gegenüber dem Evidenzgrad aus den in $>$ Abb. 2 angegebenen Gründen auf- oder abgewertet werden. Die Graduierung der Empfehlungen erfolgte außerdem über die Formulierung soll, sollte, kann ( $\triangleright$ Tab.3).

Alle Empfehlungen wurden in einem zweistufigen Konsensverfahren abgestimmt:

1. onlinebasiertes Delphiverfahren

2. abschließende Konsensuskonferenz unter Moderation

Die Konsensusstärke wurde gemäß $>$ Tab. 4 festgelegt. Im Anschluss an die Konsensuskonferenz erfolgte die finale Überarbeitung durch die AG-Leiter und die redaktionelle Zusammenstellung der Leitlinie durch die Koordinatoren.

\section{Statements}

Als Statements werden Darlegungen oder Erläuterungen von spezifischen Sachverhalten oder Fragestellungen ohne unmittelbare

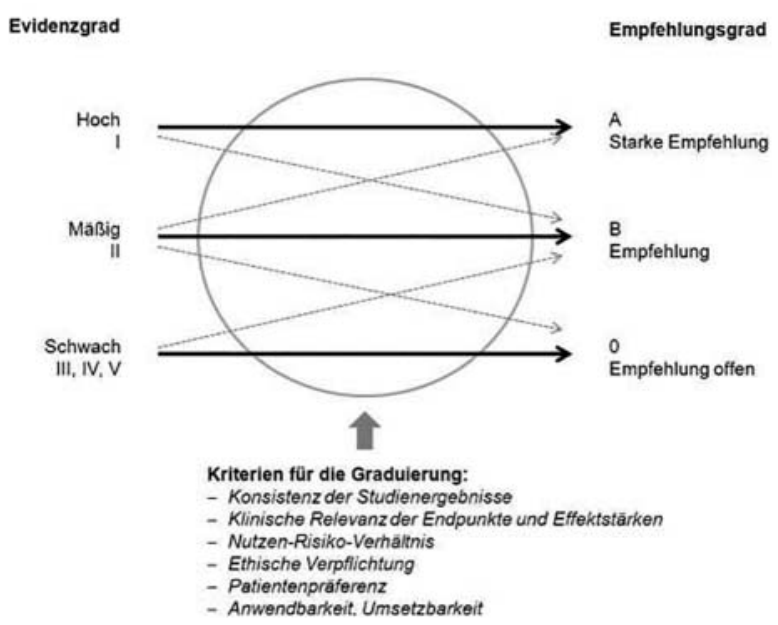

- Abb. 2 Schema der Empfehlungsgraduierung.

Handlungsaufforderung bezeichnet. Sie werden entsprechend der Vorgehensweise bei den Empfehlungen im Rahmen eines formalen Konsensusverfahrens verabschiedet und können entweder auf Studienergebnissen oder auf Expertenmeinungen beruhen.

\section{Expertenkonsens}

Als Expertenkonsens werden Empfehlungen bezeichnet, zu denen keine systematische Recherche nach Literatur durchgeführt wurde. Teilweise wurde der Expertenkonsens auch angewandt, wenn nach ausführlicher Recherche keine Literatur vorlag. Diese Empfehlungen adressieren z. T. Vorgehensweisen der guten klinischen Praxis, zu denen keine wissenschaftlichen Studien notwendig sind bzw. erwartet werden können. Für die Graduierung des Experten- 
- Tab. 3 Schema zur Graduierung von Empfehlungen.

\begin{tabular}{|l|l|l|}
\hline $\begin{array}{l}\text { Empfehlungsgrad } \\
\text { (nur S3) }\end{array}$ & Beschreibung & Syntax \\
\hline A & starke Empfehlung & soll \\
\hline B & Empfehlung & sollte \\
\hline C & offen & kann \\
\hline
\end{tabular}

- Tab.4 Einteilung der Konsensstärke.

\begin{tabular}{|l|l|}
\hline Konsens & \% Zustimmung \\
\hline Starker Konsens & $>95$ \\
\hline Konsens & $>75-95$ \\
\hline Mehrheitliche Zustimmung & $>50-75$ \\
\hline Kein Konsens & $<50$ \\
\hline
\end{tabular}

konsenses wurden keine Symbole verwendet; die Stärke der Empfehlung ergibt sich aus der verwendeten Formulierung (soll/ sollte/kann) entsprechend der Abstufung in $\mathbf{T a b} \mathbf{3}$.

Details zur Formulierung der Empfehlungen und zur strukturierten Konsensfindung sind im Leitlinienreport dargestellt.

\section{Externe Begutachtung und Verabschiedung}

Verabschiedung durch die Vorstände der herausgebenden Fachgesellschaften/Organisationen

Im Anschluss an den Peer-Review-Prozess wurde die vollständige Leitlinie von allen beteiligten Fachgesellschaften begutachtet und konsentiert.

\section{Redaktionelle Unabhängigkeit und Finanzierung der Leitlinie}

Literaturrecherche, Konferenzen und Reisekosten wurden von der DGVS finanziert. Eine finanzielle Beteiligung Dritter erfolgte nicht. Mandatsträger und Experten arbeiteten ausschließlich ehrenamtlich.

\section{Darlegung von und Umgang mit Interessenkonflikten}

Im Einklang mit dem AWMF-Regelwerk zum Umgang mit Interessenskonflikten haben alle Teilnehmer Erklärungen auf dem entsprechenden AWMF-Formular vor Beginn der Konsensuskonferenz abgegeben. Die Interessenkonflikte wurden von den Koordinatoren der Leitlinie und Frau Lynen gesichtet und der Leitliniengruppe vor Beginn der Konsensuskonferenz präsentiert. Die Mandatsträger der Leitlinie gaben eine Vielzahl von Interessenkonflikten an. Nach Einschätzung der Leitliniengruppe stellen die interdisziplinäre Besetzung der Leitliniengruppe (einschließlich stimmberechtigter Patientenvertreter) und die systematische, extern durchgeführte Literaturrecherche und Bewertung eine wichtige Maßnahme zum Ausgleich dieser Interessenkonflikte dar. Mandatsträger mit personenbezogenen Zuwendungen (Zugehörigkeit zu Advisory Boards, Gutachter- und Vortragstätigkeit) wurden daher nach kritischer Bewertung durch die Leitliniengruppe nicht von den Abstimmungen ausgeschlossen, wenn die Art der Zuwendungen nicht einseitig (z. B. Zugehörigkeit zu mehreren Advisory Boards) und die wissenschaftliche Expertise nicht verzichtbar war. Finanzielle Zuwendungen, die ausschließlich wissenschaftlichen Institutionen zugeordnet werden konnten, führten nicht zu einer Stimmenthaltung (Drittmittel, Studienbeteiligung). Mandatsträger, deren Interessenkonflikte nicht vorlagen, oder Mandatsträger mit Eigentümerinteressen (z. B. Patente, Aktienbesitz, Firmenzugehörigkeit) erhielten kein Stimmrecht. Nach Überprüfung aller Interessenkonflikte wurden keine Mandatsträger ausgeschlossen. Die Interessenkonflikte sind im Leitlinienreport veröffentlicht.

\section{Verbreitung und Implementierung}

\section{Konzept zur Verbreitung und Implementierung}

Die Leitlinie wird neben der Zeitschrift für Gastroenterologie im AWMF-Leitlinienportal (www.awmf.de) und auf der Homepage der DGVS (www.dgvs.de) veröffentlicht. Eine englische Übersetzung, ein Patientenleitfaden durch die Gastroliga sowie die DCCV (www.dccv.de) und ggfs. eine Kurzfassung sollen ebenfalls zur Verfügung gestellt werden.

\section{Gültigkeitsdauer und Aktualisierungsverfahren}

Die letzte Überarbeitung dieser Leitlinienaktualisierung erfolgte im Mai 2018. Die Gültigkeit wird auf vier Jahre geschätzt. Die Überarbeitung wird durch den Leitlinienbeauftragten der DGVS initiiert werden.

Sollte es zwischenzeitlich wichtige Neuerungen in der Diagnostik und Therapie der Colitis ulcerosa geben, die eine kurzfristige Aktualisierung notwendig erscheinen lassen, entscheiden die Leitlinienkoordinatoren (A. Dignass, T. Kucharzik) gemeinsam mit einer Aktualisierungsgruppe (B. Bokemeyer, P. Kienle, B. Siegmund, A. Stallmach) über die Notwendigkeit und die evtl. Inhalte einer Aktualisierung. Diese sollen dann online im Leitlinienportal der AWMF und auf der Homepage der DGVS veröffentlicht werden.

\section{Redaktioneller Hinweis}

\section{Geschlechtsneutrale Formulierung}

Ausschließlich zum Zweck der besseren Lesbarkeit wird auf die geschlechtsspezifische Schreibweise verzichtet. Alle personenbezogenen Bezeichnungen in diesem Dokument sind somit geschlechtsneutral zu verstehen.

\section{Partizipative Entscheidungsfindung}

Alle Empfehlungen der Leitlinie sind als Empfehlungen zu verstehen, die im Sinne einer partizipativen Entscheidungsfindung zwischen Arzt und Patient und ggf. den Angehörigen getroffen werden und umzusetzen sind. 


\section{Besonderer Hinweis}

Die Medizin unterliegt einem fortwährenden Entwicklungsprozess, sodass alle Angaben, insbesondere zu diagnostischen und therapeutischen Verfahren, immer nur dem Wissensstand zur Zeit der Drucklegung der Leitlinie entsprechen können. Hinsichtlich der angegebenen Empfehlungen zur Therapie und der Auswahl sowie Dosierung von Medikamenten wurde die größtmögliche Sorgfalt beachtet. Gleichwohl werden die Benutzer aufgefordert, die Beipackzettel und Fachinformationen der Hersteller zur Kontrolle heranzuziehen und im Zweifelsfall einen Spezialisten zu konsultieren. Fragliche Unstimmigkeiten sollen bitte im allgemeinen Interesse der OL-Redaktion mitgeteilt werden. Der Benutzer selbst bleibt verantwortlich für jede diagnostische und therapeutische Applikation, Medikation und Dosierung.

In dieser Leitlinie sind eingetragene Warenzeichen (geschützte Warennamen) nicht besonders kenntlich gemacht. Es kann also aus dem Fehlen eines entsprechenden Hinweises nicht geschlossen werden, dass es sich um einen freien Warennamen handelt.

Das Werk ist in allen seinen Teilen urheberrechtlich geschützt. Jede Verwertung außerhalb der Bestimmung des Urhebergesetzes ist ohne schriftliche Zustimmung der DGVS unzulässig und strafbar. Kein Teil des Werks darf in irgendeiner Form ohne schriftliche Genehmigung reproduziert werden. Dies gilt insbesondere für Vervielfältigungen, Übersetzungen, Mikroverfilmungen und die Einspeicherung, Nutzung und Verwertung in elektronischen Systemen, Intranets und dem Internet.

\section{Diagnostik}

\section{Klassifikation}

\section{EMPFEHLUNG 2.1}

Eine Klassifikation bezüglich der Ausdehnung der Erkrankung soll erfolgen. Es soll eine endoskopische Einteilung in die Proktitis (begrenzt auf das Rektum), die Linksseitencolitis (Ausdehnung bis zur linken Flexur) und die ausgedehnte Colitis erfolgen.

Expertenkonsens, starke Empfehlung, starker Konsens

\section{Hintergrund}

Eine Klassifikation der Colitis ulcerosa (CU) nach der Ausdehnung der Erkrankung ist aus zwei wesentlichen Gründen sinnvoll. Einerseits entscheidet die Lokalisation der Erkrankung über den Einsatz von topischer und/oder systemischer Applikation der Medikation, insbesondere der 5-ASA-Präparate. Bei einer Proktitis werden bevorzugt Suppositorien eingesetzt, bei der Linksseitencolitis können Einläufe oder Schaumpräparate zum Einsatz kommen. Bei ausgedehnter Colitis ulcerosa sollten oral verabreichte Substanzen verwendet werden, nach der Studie von Marteau allerdings idealerweise kombiniert mit einer zusätzlichen topischen 5-ASA-Therapie [1]. Weiterhin hat die Ausdehnung Einfluss auf den Beginn des Karzinomüberwachungsprogramms. Aus diesem Grund wird die Empfehlung zur Vorsorgekoloskopie
Tab. 5 Ausdehnung der Colitis ulcerosa (nach Silverberg et al. [2])

\begin{tabular}{|l|l|l|}
\hline Einteilung & Ausdehnung & Beschreibung \\
\hline E1 & Proktitis & $\begin{array}{l}\text { Limitiert auf das Rektum } \\
\text { (distal des rektosigmoidalen } \\
\text { Übergangs) }\end{array}$ \\
\hline E2 & Linksseitencolitis & Befall bis zur linken Flexur \\
\hline E3 & Ausgedehnte Colitis & $\begin{array}{l}\text { Ausdehnung über die linke } \\
\text { Flexur hinaus bis zur Pancolitis }\end{array}$ \\
\hline
\end{tabular}

entsprechend der Ausdehnung der Erkrankung differenziert ausgesprochen (siehe 2.28-2.31). Die bevorzugte Einteilung der Colitis ulcerosa unterscheidet die Proktitis, die Linksseitencolitis sowie die ausgedehnte Colitis mit Ausdehnung über die linke Flexur hinaus (Montreal-Klassifikation) [2] ( Tab. 5).

\section{EMPFEHLUNG 2.2}

Das gleichzeitige Vorliegen einer PSC soll dokumentiert werden, da dies die endoskopische Überwachungsstrategie beeinflusst.

Expertenkonsens, starke Empfehlung, starker Konsens

\section{Hintergrund}

Die Dokumentation einer primär sklerosierenden Cholangitis (PSC) ist von Bedeutung, da ihr Vorliegen mit einem deutlich erhöhten Risiko für die Entwicklung eines kolorektalen Karzinoms vergesellschaftet ist [3,4]. Diese Tatsache hat Einfluss auf die Empfehlungen für das Kolonkarzinomüberwachungsprogramm bei gleichzeitigem Vorliegen von Colitis ulcerosa und PSC (siehe 2.32).

\section{Krankengeschichte}

\section{EMPFEHLUNG 2.3}

Die Anamnese sollte eine detaillierte Erfragung über Art und Beginn der Symptome, eine kürzliche Reiseanamnese, Nahrungsmittelunverträglichkeiten, Kontakte mit infektiösen Durchfallerkrankungen, Impfstatus, Raucheranamnese, Familienanamnese und die Medikamentenanamnese (insbesondere auch bezüglich Antibiotika und nichtsteroidaler Antirheumatika) beinhalten. Weiterhin sollte die Anamnese Fragen bezüglich extraintestinaler Manifestationen (Mund, Haut, Augen und/oder Gelenke) sowie nach perianalen Abszessen, Fisteln und Analfissuren beinhalten.

Expertenkonsens, Empfehlung, Konsens

\section{Hintergrund}

Selbstverständlich gehört eine ausführliche Anamnese und körperliche Untersuchung zur täglichen Routine in der Inneren Medizin und ist nicht spezifisch für Patienten mit Colitis ulcerosa. 
Nichtsdestotrotz gibt es bestimmte Aspekte, die bei der Colitis ulcerosa (bzw. den chronisch-entzündlichen Darmerkrankungen) eine besondere Rolle spielen und eine besondere Aufmerksamkeit verdienen.

Die Unterscheidung zwischen Morbus Crohn und Colitis ulcerosa kann manchmal schwierig bzw. unmöglich sein. Das Fehlen von rektalem Blutabgang oder Symptomen bei aktiven Rauchern sollte eher an einen Morbus Crohn denken lassen. Eine infektiöse oder medikamenteninduzierte Colitis sollte anamnestisch, soweit möglich, abgegrenzt werden. Die Einnahme von nichtsteroidalen Antirheumatika (NSAR) scheint ein signifikantes Risiko zu beinhalten, eine bestehende Colitis ulcerosa zu verschlechtern [5 - 8].

Knapp die Hälfte der Patienten mit Colitis ulcerosa erlebt einen Verlauf der Erkrankung, der eine immunsuppressive Therapie mit Steroiden, Thiopurinen oder TNF-Antikörpern erfordert [9, 10]. Das Risiko für opportunistische Infektionen unter immunsuppressiver Therapie, insbesondere unter Mehrfachimmunsuppression, ist signifikant erhöht [11]. Daher wird sowohl in einem Konsensuspapier der ECCO als auch im vorliegenden Konsensuspapier der DGVS die Erhebung und ggf. Vervollständigung des Impfstatus gefordert.

Aktives Rauchen hat einen protektiven Effekt auf die Entwicklung und den Schweregrad einer Colitis ulcerosa [12, 13]. Ex-Raucher hingegen haben ein $70 \%$ höheres Risiko, eine Colitis ulcerosa zu entwickeln; diese weist häufiger einen refraktären und ausgedehnten Verlauf auf, interessanterweise auch im Vergleich zu Patienten, die nie geraucht haben. Krankenhausaufenthalte und Kolektomieraten sind bei Ex-Rauchern ebenfalls höher als bei Patienten, die nie geraucht haben $[14,15]$. Ex-Raucher, die wieder beginnen zu rauchen, scheinen einen milderen Verlauf der Erkrankung zu erleben [16, 17]. Umstritten ist die Frage, ob Rauchen auch das Auftreten einer PSC oder das Auftreten einer Pouchitis nach Kolektomie und ileoanaler Pouchanlage verhindern kann $[18,19]$. Ein weiterer Zusammenhang scheint zwischen Appendektomie und der Entwicklung einer Colitis ulcerosa zu bestehen. Kohortenstudien und eine Metaanalyse weisen darauf hin, dass eine Appendektomie in der Kindheit (wegen „echter“ Appendizitis) einen protektiven Effekt (69\% Risikoreduktion) bezüglich des Auftretens und des Schweregrads einer späteren Colitis ulcerosa hat. Auch diese Daten sind nicht in allen Folgestudien bestätigt worden [16, 20 - 25]. Dabei scheint der protektive Effekt der Appendektomie zusätzlich zum Effekt des Rauchens zu bestehen, während die Appendektomie nicht vor der Entwicklung einer PSC schützt. Eine Appendektomie nach dem Auftreten einer Colitis ulcerosa scheint keinen wesentlichen positiven Effekt mehr zu haben. Die Daten zur Appendektomie sind epidemiologisch und pathophysiologisch interessant, sie haben im klinischen Alltag allerdings keine therapeutische Konsequenz und werden daher in den Empfehlungen nicht berücksichtigt.

Die Familienanamnese hat einen besonderen Stellenwert in der Anamnese. Erstgradige Verwandte von Patienten mit Colitis ulcerosa haben ein 10- bis 15-fach erhöhtes Risiko, ebenfalls an einer Colitis ulcerosa zu erkranken [17]. Allerdings beträgt das lebenslange Risiko für erstgradige Verwandte, eine Colitis ulcerosa zu entwickeln, nur ungefähr $5 \%$ (oder umgekehrt $95 \%$, nicht an einer Colitis ulcerosa zu erkranken). Dieses ist eine wichtige Information für Patienten bei der Familienplanung. Familiäre Fälle von
Colitis ulcerosa scheinen eher das weibliche Geschlecht zu betreffen, weiterhin scheint das Erkrankungsalter niedriger zu liegen im Vergleich zu sporadischen Fällen [26].

\section{EMPFEHLUNG 2.4}

Bei Erstdiagnose und bei Auftreten spezifischer Symptome sollen eine komplette körperliche Untersuchung inklusive einer oralen und perianalen Inspektion und die Beachtung eventuell vorliegender extraintestinaler Manifestationen erfolgen. Eine rektale Untersuchung soll spätestens im Rahmen der Koloskopie durchgeführt werden. Expertenkonsens, starke Empfehlung, Konsens

\section{Hintergrund}

Die körperliche Untersuchung ist bei Patienten mit Colitis ulcerosa in Abwesenheit extraintestinaler Manifestationen wenig spezifisch. Die klinische Symptomatik insbesondere mit Durchfällen, Tenesmen und rektalem Blutabgang ist häufig wegweisend. Bei schwerem Schub können Tachykardie, Gewichtsverlust, abdominelle Abwehrspannung und/oder abgeschwächte Darmgeräusche auftreten. Viel diskutiert, sowohl in der Arbeitsgruppe als auch im Plenum, wurde die Notwendigkeit einer perianalen Inspektion sowie der rektalen Untersuchung. Selbstverständlich ist sie nicht bei jeder Vorstellung des Patienten notwendig, sie wird in der Regel im Rahmen einer Koloskopie erfolgen. Allerdings soll hier nochmals auf das erhöhte Risiko (kolo)rektaler Karzinome bei Colitis ulcerosa hingewiesen werden, sodass die Dokumentation der rektalen Untersuchung gerechtfertigt scheint. Explizit sollte nach extraintestinalen Manifestationen insbesondere an Augen, Mund, Gelenken und Haut sowie nach perianalen Manifestationen gefragt werden [27].

\section{EMPFEHLUNG 2.5}

Bei Kindern und Jugendlichen sollen zusätzlich die Entwicklung von Gewicht, Länge und das Pubertätsstadium bei Erstdiagnose und regelmäßig im Krankheitsverlauf erfasst werden.

Expertenkonsens, starke Empfehlung, starker Konsens

\section{Hintergrund}

Bei Kindern und Jugendlichen ist zur Diagnose von Wachstumsverzögerungen bei jeder Visite der z-Score oder das Längensollgewicht im Vergleich zu nationalen geschlechtsspezifischen Referenzwerten zu erheben. Bei Wachstumsretardierung ist durch eine Röntgenaufnahme der nicht dominanten Hand das Knochenalter zu bestimmen. Weiterhin sollte das Pubertätsstadium nach Tanner bestimmt werden [28]. 


\section{EMPFEHLUNG 2.6}

Die Diagnose einer Colitis ulcerosa soll auf dem Boden einer Kombination von Anamnese, klinischer Untersuchung und typischen laborchemischen, sonografischen, endoskopischen und histologischen Befunden gestellt werden.

Expertenkonsens, starke Empfehlung, Konsens

\section{EMPFEHLUNG 2.7}

Bei Zweifel bezüglich der Diagnose sollte die Endoskopie inklusive Histologiegewinnung im Intervall (z. B. nach 3 6 Monaten) wiederholt werden.

Evidenzgrad 4, Empfehlungsgrad B, Konsens

\section{Hintergrund}

Der natürliche Verlauf der Colitis ulcerosa ist charakterisiert durch Episoden von Krankheitsschüben, die sich mit Phasen der Remission abwechseln. Zu Beginn der Diagnose kann die Abgrenzung zu einer infektiösen Colitis schwierig sein und erst der Verlauf zeigt den chronischen Charakter der Erkrankung (siehe unten). Selten (nur ca. $5 \%$ der Patienten) kann der Krankheitsverlauf auch kontinuierlich ohne intermittierende Remissionsphasen verlaufen. Ebenso häufig kann sich die Colitis ulcerosa auch als einzelner Schub mit anschließender prolongierter andauernder Remission präsentieren [29]. Eine zügige Etablierung der Diagnose inklusive Ausdehnung und Schweregrad des Schubs ermöglicht eine optimale Therapiestrategie. Ein Goldstandard für die Diagnosestellung der Colitis ulcerosa existiert allerdings nicht. Die Diagnose beruht auf der Kombination typischer Befunde in der Anamnese, Endoskopie, aus sonografisch/radiologischen Techniken und der Histopathologie. Die pathomorphologischen Kriterien ergeben sich aus der Biopsieentnahme bei der Endoskopie oder aus der Aufarbeitung von Operationspräparaten. Normalbefunde in der Histopathologie der Schleimhautbiopsien schließen eine aktive Colitis ulcerosa aus. Nach Etablierung der Diagnose ändert sich bei ca. $10 \%$ der Patienten innerhalb der ersten 5 Jahre nach Diagnosestellung die Diagnose zu einem Morbus Crohn, oder die Diagnose einer chronisch entzündlichen Darmerkrankung wird insgesamt verworfen. Daher ist, insbesondere bei Zweifeln an der Diagnose, die endoskopische und histopathologische Bestätigung durch eine erneute Endoskopie mit Histologiegewinnung geboten [30]. Bei einer Minderheit der Patienten wird es auch im Verlauf nicht möglich sein, die exakte Zuordnung zu den Entitäten Colitis ulcerosa und Morbus Crohn vorzunehmen, dann spricht man von der sogenannten „indeterminierten Colitis“ (oder nach der Montreal Working Party 2005: Inflammatory Bowel Disease unclassified (IBDu) [2, 31].

\section{Diagnosestellung}

\section{EMPFEHLUNG 2.8}

Die initiale Labordiagnostik sollte neben dem Blutbild mindestens folgende Parameter enthalten: Entzündungsstatus, Eisenhaushalt, Nierenfunktion, Transaminasen und Cholestaseparameter.

Evidenzgrad 4, Empfehlungsgrad B, starker Konsens

\section{EMPFEHLUNG 2.9}

Für die begleitende laborchemische Diagnostik eines Ansprechens auf die Therapie können CRP und/oder fäkale Neutrophilenmarker als laborchemische Verlaufsparameter herangezogen werden.

Evidenzgrad 2, Empfehlungsgrad 0, mehrheitliche Zustimmung

\section{Hintergrund}

Bei jedem Patienten mit aktiver Colitis ulcerosa sollten als minimale Labordiagnostik ein Blutbild, inflammatorische Marker (CRP), Parameter des Eisenhaushalts, Nierenretentionsparameter, Transaminasen und Cholestaseparameter erhoben werden. Dabei können die Laborwerte, insbesondere bei milder bis moderater Colitis ulcerosa und/oder distalem Befallsmuster, durchaus normal ausfallen. Ausgenommen bei der Proktitis, bei der in der Regel keine auffälligen Laborwerte zu erwarten sind, korreliert das C-reaktive Protein mit der Ausdehnung der Erkrankung sowie schwach mit der klinischen Aktivität [32, 33]. Der CRP-Anstieg fällt bei Patienten mit Colitis ulcerosa in der Regel geringer aus als bei Patienten mit Morbus Crohn, kann jedoch bei CRP-positiven Patienten durchaus auch als Marker für die klinische und endoskopische Krankheitsaktivität herangezogen werden [34]. Bei Patienten mit schwerer Krankheitsaktivität ist in der Regel auch die BSG erhöht, und es besteht eine Anämie. Die Wertigkeit fäkaler Stuhlmarker und insbesondere von Calprotectin als Marker für die klinische und endoskopische Entzündungsaktivität bei Colitis ulcerosa konnte in mehreren Studien herausgestellt werden [35-38]. Allerdings können naturgemäß weder serologische Marker wie das CRP noch fäkale Entzündungsmarker die Colitis ulcerosa von einer infektiösen Ursache differenzieren. Zwei kleine Studien berichten über einen Stellenwert von Procalcitonin zur Abgrenzung selbstlimitierender Kolitiden [39, 40]. Insbesondere bei der Erstdiagnostik sind zur Abgrenzung und zur Diagnostik von selbstlimitierenden infektiösen Kolitiden Stuhlkulturen hilfreich [41, 42] (siehe Empfehlung 2.10).

Bezüglich des Eisenhaushalts ist das Ferritin nur bedingt hilfreich, da es im Schub als Akutphaseprotein erhöht sein kann und damit keinen Rückschluss auf die Eisenspeicher zulässt. Daher sollte im Zweifel die Bestimmung der Transferrinsättigung bzw. des löslichen Transferrinrezeptors hinzugezogen werden [43-46].

Die routinemäßige Bestimmung von perinukleären antineutrophilen cytoplasmatischen Antikörpern (pANCA) für die Colitis ulcerosa und Anti-Saccharomyces-cerevisiae-Antikörpern (ASCA) 
für den Morbus Crohn zur Differenzierung der beiden Krankheitsentitäten wird wegen der niedrigen Sensitivität nicht empfohlen [47], kann allerdings im Einzelfall hilfreich sein. In den meisten Publikationen werden pANCA bei maximal $65 \%$ der Patienten mit Colitis ulcerosa und in weniger als $10 \%$ der Patienten mit Morbus Crohn beschrieben [48, 49]. Eine große Metaanalyse mit Berücksichtigung von 60 Studien konnte zeigen, dass die Sensitivität für die Detektion eines Morbus Crohn bei der Konstellation ASCA+/pANCA- bei 55 \% liegt (Spezifität $93 \%$ ), während die Sensitivität für die Detektion einer Colitis ulcerosa bei der Konstellation pANCA+ bei $55 \%$ (Spezifität $89 \%$ ) und bei pädiatrischen Patienten sogar noch höher liegt (70\% bzw. $93 \%$ ) [50].

\section{EMPFEHLUNG 2.10}

Eine intestinale Infektion sollte bei Erstdiagnostik und bei einer Schubsymptomatik im Verlauf ausgeschlossen werden. Evidenzgrad 4, Empfehlungsgrad B, Konsens

Bei der Erstdiagnostik sollte eine mikrobiologische Stuhldiagnostik auf bakteriell infektiöse Erreger inklusive Clostridiumdifficile-Toxin erfolgen.

Evidenzgrad 2, Empfehlungsgrad B, Konsens

Bei Patienten mit entsprechender Reiseanamnese sollte eine ergänzende Diagnostik bezüglich landestypischer Erreger durchgeführt werden.

Evidenzgrad 4, Empfehlungsgrad B, Konsens

\section{Hintergrund}

In der initialen Diagnostik ist die Abgrenzung gegenüber infektiösen, in der Regel selbst limitierten Ursachen wichtig. Stuhlproben sollten untersucht werden auf die häufigen Erreger inklusive Campylobacter spp, Escherichia coli 0157:H7 und Clostridium difficile toxin A und B. Bei entsprechender Anamnese können spezielle Stuhluntersuchungen sinnvoll sein, wie die Stuhlmikroskopie und Stuhlantigendiagnostik auf Amöben oder andere Parasiten. Auf die spezifische Diagnostik wird ausführlich im Infektionskapitel eingegangen.

\section{EMPFEHLUNG 2.11}

Bei etablierter Colitis ulcerosa soll bei schwerem Schub und bei therapierefraktärem Verlauf bzw. vor Intensivierung einer immunsuppressiven Therapie eine mikrobiologische Diagnostik inklusive Untersuchungen auf Clostridium-difficileToxin und Cytomegalievirus erfolgen.

Evidenzgrad 2, Empfehlungsgrad A, starker Konsens

\section{Hintergrund}

Im weiteren Verlauf muss nicht bei jedem Schub die Stuhldiagnostik wiederholt werden $[42,51]$; allerdings sollte insbesondere die Diagnostik auf C. difficile [52 - 54] und Cytomegalievirus(CMV)(Re-)Infektionen [55 - 58] bei schweren oder refraktären Verläufen erfolgen oder falls anamnestisch dem Schub eine Einnahme von Antibiotika vorausgegangen ist (siehe auch Kapitel 4: Infektionen).

\section{EMPFEHLUNG 2.12}

Die quantitative Bestimmung von fäkalen Neutrophilenmarkern (z. B. Calprotectin) sollte in der klinischen Differenzialdiagnostik zur Abgrenzung der Beschwerden gegenüber einer (funktionellen) Reizdarmsymptomatik genutzt werden. Evidenzgrad 2, Empfehlungsgrad B, Konsens

\section{EMPFEHLUNG 2.13}

Zur Verlaufsdiagnostik bei etablierter Colitis ulcerosa sollte die quantitative Bestimmung von fäkalen Neutrophilenmarkern im Stuhl herangezogen werden.

Evidenzgrad 1, Empfehlungsgrad B, starker Konsens

\section{Hintergrund}

Verschiedene fäkale Entzündungsmarker wie Calprotectin, Lysozym, PMN-Elastase, Lactoferrin und S100A12 sind bei chronisch-entzündlichen Darmerkrankungen untersucht worden [59-62]. Calprotectin und Lactoferrin scheinen dabei die höchste Sensitivität für eine intestinale Entzündung aufzuweisen und korrelieren mit dem klinischen und endoskopischen Entzündungsgrad der Colitis ulcerosa [63, 64]. Fäkale Marker sind nicht in der Lage, zwischen verschiedenen Ursachen einer intestinalen Entzündung zu unterscheiden. Aus diesem Grund ist der diagnostische Nutzen in der Primärdiagnostik der Colitis ulcerosa limitiert. Allerdings können fäkale Marker zur Abgrenzung funktioneller Beschwerden und insbesondere in der pädiatrischen Diagnostik hilfreich sein. In mehreren Untersuchungen konnte belegt werden, dass sowohl pädiatrische als auch erwachsene Patienten mit chronisch-entzündlichen Darmerkrankungen signifikant höhere fäkale Calprotectinwerte aufweisen als die Normalbevölkerung und als Patienten mit Reizdarmsyndrom. Die Marker können daher sehr gut als Differenzierungsmarker zum Reizdarmsyndrom bzw. zu nichtentzündlichen Ursachen intestinaler Beschwerden eingesetzt werden, insbesondere in der Pädiatrie [65-68].

Darüber hinaus konnte in mehreren Studien gezeigt werden, dass u. a. Calprotectin als Marker für die Früherkennung von Rezidiven bei Colitis ulcerosa herangezogen werden kann, da endoskopische Rezidive mit hoher Sensitivität und Spezifität vor Auftreten einer klinischen Symptomatik erkannt werden [37, 38, 69, 70]. Im klinischen Alltag können die fäkalen Neutrophilenmarker daher hilfreich sein, um Beschwerden der Patienten besser einordnen zu können. Aus dem individuellen longitudinalen Profil der Neutrophilenmarker können Rückschlüsse auf die Aktivität der Erkrankung gezogen werden.

\section{Endoskopische Diagnostik}

\section{EMPFEHLUNG 2.14}

Bei Verdacht auf Colitis ulcerosa sollte eine lleokoloskopie mit Biopsien aus dem term. Ileum und allen Kolonsegmenten unter Einschluss des Rektums (zumindest zwei Biopsien/ 
Segment; Einsendung in getrennten Probengefäßen) erfolgen, um die Diagnose zu stellen und die Ausdehnung der Erkrankung festzustellen.

Evidenzgrad 4, Empfehlungsgrad B, Konsens

\section{Hintergrund}

Die komplette Koloskopie mit Intubation des terminalen Ileums und segmentaler Entnahme von Darmbiopsien wird bei Patienten mit Verdacht auf Colitis ulcerosa in der Erstdiagnostik einer Sigmoidoskopie vorgezogen, um einerseits das Befallsmuster klassifizieren zu können und andererseits eine lleitis terminalis Crohn weitgehend auszuschließen [71, 72]. Dieses Vorgehen erscheint kosteneffektiver zu sein als eine Indexsigmoidoskopie $[73,74]$.

Im Rahmen der Erstdiagnostik sollten zumindest jeweils 2 Stufenbiopsien aus dem Ileum und aus allen Kolonabschnitten unter Einbeziehung des Rektums gewonnen werden und die bioptischen Proben bezüglich ihrer Lokalisation getrennt gekennzeichnet sein.

Eine wiederholte endoskopische Diagnostik mit entsprechender histopathologischer Begutachtung im Intervall kann bei unklaren Fällen notwendig sein. Bei ca. 10 \% der Patienten wurde eine Rücknahme der Diagnosestellung einer Colitis ulcerosa bzw. eine Änderung in einen Morbus Crohn innerhalb der ersten fünf Jahre beschrieben [75]. Bei Patienten mit akuter, schwerer Colitis wird die Durchführung einer kompletten Koloskopie nicht empfohlen und sollte zunächst durch eine Sigmoidoskopie ersetzt werden.

\section{EMPFEHLUNG 2.15}

Eine routinemäßige Koloskopie sollte bei Patienten mit Colitis ulcerosa in der Remission bis zum Beginn der Karzinomüberwachung nicht erfolgen.

Expertenkonsens, Empfehlung, starker Konsens

Eine erneute endoskopische Diagnostik in der klinischen Remission kann zur Beurteilung des Therapieansprechens und unter Immunsuppression oder biologischer Therapie unter dem Aspekt einer Therapiedeeskalation erwogen werden. Expertenkonsens, Empfehlung offen, starker Konsens

\section{EMPFEHLUNG 2.16}

Eine endoskopische Evaluation kann bei therapierefraktären Verläufen zur Bestätigung der Aktivität der Erkrankung und zum Ausschluss von infektiösen oder anderen Komplikationen durchgeführt werden.

Expertenkonsens, Empfehlung offen, Konsens

\section{Hintergrund}

Trotz der Wichtigkeit zur Beurteilung der Krankheitsausdehnung bei der Festlegung der Prognose, der Notwendigkeit zur Überwa- chung und der Wahl der Therapie wurde bisher noch nicht die Angemessenheit regelmäßiger Wiederholungsuntersuchungen nach einer Indexkoloskopie untersucht. Eine medikamentös induzierte klinische Remission muss nicht zwingend mit einer endoskopisch oder histologisch erfassten Remission einhergehen [76]. Es gibt andererseits zahlreiche Hinweise, dass eine sogenannte Mukosaheilung mit einem günstigeren klinischen Ausgang assoziiert ist [77]. In systematischen Reviews konnte eine Assoziation zwischen der Mukosaheilung und der Vermeidung einer Kolektomie, dem Erreichen einer steroidfreien und klinischen Remission nachgewiesen werden $[78,79]$, daher kann eine endoskopische Kontrolle nach einer medikamentösen Remissionsinduktion erwogen werden.

Gleichsam kann der Mukosaaspekt auch in den Entscheidungsprozess einer Therapiedeeskalation bei stabiler klinischer Remission unter Immunsuppression/ Biologika-Therapie herangezogen werden. Auch wenn die Datenlage bei der Colitis ulcerosa diesbezüglich unzureichend ist, sind die Ergebnisse bei Morbus Crohn vielversprechend [80].

Zur Bestimmung der klinischen oder endoskopischen Entzündungsaktivität stehen zahlreiche Indizes zur Verfügung. In der täglichen Routine werden diese Indizes selten eingesetzt, finden ihre Anwendung aber im Rahmen von klinischen Studien. Zur Evaluation der endoskopischen Inflammation wird oftmals der endoskopische Mayo Score verwendet [81]. Den einzig validierten endoskopischen Aktivitätsindex stellt der Ulcerative Colitis Endoscopic Index of Severity (UCEIS) dar [82].

Klinisch wird vor allem die Klassifikation einer schweren Colitis ulcerosa nach Truelove und Witts aus dem Jahr 1955 [83] bis heute weitgehend verwendet, da sie einfach zu merken und anzuwenden ist. Diese Klassifikation wird immer noch als das Verfahren der Wahl angesehen, um Patienten zu identifizieren, die eine sofortige Krankenhauseinweisung und eine intensive Therapie benötigen [84]. Für pädiatrische Patienten hat sich der PUCAI nach Turner etabliert [85].

\section{Differenzialdiagnostische Abgrenzung zum Morbus Crohn}

\section{EMPFEHLUNG 2.17}

Bei nicht eindeutig zu klassifizierender Colitis sollte eine Diagnostik des oberen Gastrointestinaltrakts mittels Ösophagogastroduodenoskopie (mit Biopsien) und des mittleren Gastrointestinaltrakts mittels MRT des Dünndarms und/oder abdomineller Sonografie durchgeführt werden. Expertenkonsens, Empfehlung, starker Konsens

\section{Hintergrund}

Bei diagnostischen Unklarheiten (z. B. Aussparung des Rektums, ungewöhnliche Symptome, endoskopische Hinweise für eine Backwash-Ileitis) sollte differenzialdiagnostisch das Vorliegen eines Morbus Crohn bedacht werden und in Abhängigkeit vom klinischen Kontext eine entsprechende Diagnostik des oberen und mittleren Verdauungstrakts durchgeführt werden. Dabei sollte analog zu den bestehenden DGVS-Leitlinien zur Diagnostik und Therapie des Morbus Crohn verfahren werden [86]. 


\section{Ultraschall}

\section{EMPFEHLUNG 2.18}

Die hochauflösende abdominelle Sonografie sollte Bestandteil der Diagnostik bei der Erstdiagnose und in der Verlaufsdiagnostik sowie beim schweren akuten Schub zur Erfassung von Komplikationen sein.

Evidenzgrad 2, Empfehlungsgrad B, starker Konsens

\section{Hintergrund}

Der transabdominelle Ultraschall kann mit einer Sensitivität von bis zu 90 \% Entzündungen im Bereich des Dickdarms detektieren. Die Sonografie hat den Vorteil, preiswert und nicht invasiv zu sein. Wie alle anderen diagnostischen Erfahren auch ist die Genauigkeit des Verfahrens abhängig von der Erfahrung des Untersuchers. Darüber hinaus besteht eine geringe Spezifität zur Differenzierung einer Colitis ulcerosa von anderen Ursachen einer Dickdarmentzündung [87 - 89]. In der Hand des geübten Untersuchers kann sowohl die Aktivität der Erkrankung als auch die Krankheitsausdehnung zuverlässig bestimmt werden [90-96]. Es besteht eine gute Korrelation der Sonografie mit der endoskopischen Aktivität und durch die gute Korrelation mit dem Therapieansprechen kann die Methode auch als prognostisches Verfahren angewandt werden [97]. Die Dopplersonografie der Arteria mesenterica superior und inferior wurde angewandt, um die Krankheitsaktivität und das Risiko eines Rezidivs zu evaluieren. Sie sollte jedoch aufgrund der unzureichenden Datenlage und der geringen flächendeckenden Nutzung zum gegenwärtigen Zeitpunkt nicht als Standardverfahren verstanden werden [98, 99]. Für die kontrastverstärkte Ultraschalluntersuchung (CEUS) wurde eine Korrelation mit der histologischen Entzündungsaktivität nachgewiesen, aufgrund der zum gegenwärtigen Zeitpunkt unzureichenden Datenlage kann allerdings keine Empfehlung zur Anwendung als Standardverfahren gegeben werden [100].

\section{Kolonstenose bei Colitis ulcerosa}

\section{EMPFEHLUNG 2.19}

Da das Vorliegen einer Kolonstenose bei Colitis ulcerosa malignitätsverdächtig ist, sollte eine ausgiebige Biopsieentnahme aus dem Bereich der Stenose und zusätzlich eine bildgebende Diagnostik (z. B. CT, MRT) erfolgen. Bei unklarer Dignität einer Kolonstenose sollte die Entscheidung zur Operation großzügig gestellt werden.

Evidenzgrad 4, Empfehlungsgrad B, Konsens

\section{Hintergrund}

Bei langjähriger Colitis ulcerosa ist eine Kolonstriktur bzw. -stenose als ein Hinweis auf das Vorliegen eines kolorektalen Karzinoms zu interpretieren; dies erfordert daher eine histologische Abklärung [101]. Eine definitive endoskopisch-histologische Klärung ist wegen des submukösen Wachstums des mit Colitis ulcerosa assoziierten Karzinoms häufig schwierig [102, 103]. Aus dem Grund wird eine entsprechende Empfehlung zur großzügigen Stellung einer OP-Indikation als gerechtfertigt angesehen. Wenn eine Koloskopie aufgrund einer Stenose bzw. Striktur inkomplett ist, sollte eine CT- oder MR-Kolonografie durchgeführt werden. Eine CT-Kolonografie oder eine MR-Kolonografie kann die Schleimhautbeschaffenheit und Ausdehnung der Colitis proximal der Striktur identifizieren, möglicherweise jedoch nicht alle Läsionen, die im Rahmen einer Koloskopie sichtbar sind, zur Darstellung bringen [104, 105]. In einer retrospektiven Kohortenanalyse bei Patienten mit einer Kolonstenose bei Morbus Crohn und bei Colitis ulcerosa, die präoperativ keine Malignitätszeichen aufwiesen, wurde in 7,8\% der Fälle postoperativ bei den Colitis-ulcerosa-Patienten ein Kolonkarzinom diagnostiziert [106]. Bei unklaren Befunden sollte daher die chirurgische Resektion erfolgen. Trotz der reduzierten Evidenzlage wurde hier diese Empfehlung ausgesprochen, um jede Verzögerung einer Operation angesichts eines möglichen Kolonkarzinoms zu vermeiden [106].

\section{Pädiatrie}

\section{EMPFEHLUNG 2.20}

Die Diagnose einer Colitis ulcerosa sollte bei Kindern bei Vorliegen von chronischen (> 4 Wochen) oder rezidivierenden (> 2 Episoden innerhalb von 6 Monaten) blutigen Durchfällen nach Ausschluss einer infektiösen Genese in Betracht gezogen werden. Evidenzgrad 4, Empfehlungsgrad B, Konsens

\section{Hintergrund}

Kinder mit einer Colitis ulcerosa weisen in der Regel typische Symptome wie Anämie (84\%), chronische Diarrhö (74\%) und abdominelle Schmerzen, vorwiegend in Form von Tenesmen (62\%), auf [107]. Bei chronischen (>2 Wochen) oder rezidivierenden Durchfällen sollte auch ohne Blutbeimengungen eine Abklärung erfolgen. Gewichtsverlust ist weniger typisch bei der Colitis ulcerosa (35\%) als beim Morbus Crohn (58\%). Das häufigste extraintestinale Symptom stellen Arthropathien dar (10\%). Hautmanifestationen sind selten. Gegenüber erwachsenen Patienten liegt in drei Vierteln der Fälle eine ausgedehnte Colitis ulcerosa vor, während ein distaler Befall die Ausnahme darstellt. Über 50 \% der Patienten mit Colitis ulcerosa weisen jährlich mindestens ein Rezidiv auf. Die Diagnose einer chronisch-entzündlichen Darmerkrankung wird bestätigt durch klinische Evaluation und eine Kombination von biochemischen, endoskopischen, histologischen und bei Verdacht auf Morbus Crohn auch radiologischen (MRT-Enterografie oder -Enteroklysma) Untersuchungen. Die diagnostischen Kriterien entsprechen denen bei erwachsenen Patienten [108].

\section{EMPFEHLUNG 2.21}

Die initiale Diagnostik bei Kindern und Jugendlichen mit Verdacht auf eine chronisch-entzündliche Darmerkrankung soll eine Ileokoloskopie mit Entnahme von Stufenbiopsien aus dem term. Ileum und allen Kolonsegmenten beinhalten. Evidenzgrad 4, Empfehlungsgrad A, starker Konsens 
Im gleichen Untersuchungsgang sollte eine Ösophagogastroduodenoskopie mit Entnahme von Stufenbiopsien erfolgen. Evidenzgrad 4, Empfehlungsgrad B, starker Konsens

\section{Hintergrund}

Für das diagnostische Vorgehen wurden von einer Arbeitsgruppe der European Society of Pediatric Gastroenterology, Hepatology and Nutrition (ESPGHAN) Empfehlungen festgelegt [109]. Kinder mit Verdacht auf eine chronisch-entzündliche Darmerkrankung sollen eine komplette Koloskopie mit Intubation des terminalen Ileums und Entnahme von Stufenbiopsien (terminales Ileum, Coecum, Colon ascendens, Colon transversum, Colon descendens, Sigma und Rektum) sowie eine Ösophagogastroduodenoskopie mit Stufenbiopsien aus Ösophagus, Magen und Duodenum erhalten [109]. Nur bei eindeutiger Zuordnung zur Colitis ulcerosa kann auf eine Bildgebung des Dünndarms (in der Regel eine MRT-Enterografie) verzichtet werden. Im Unterschied zu erwachsenen Patienten haben drei Viertel der pädiatrischen Patienten eine ausgedehnte Colitis, sodass eine komplette Koloskopie obligat ist. Eine alleinige Sigmoidoskopie ist in der Regel nicht indiziert, mit Ausnahme einer schweren Colitis ulcerosa, bei der das Risiko einer Darmperforation erhöht ist. Die Endoskopie sollte bei pädiatrischen Patienten in Vollnarkose oder tiefer Analgosedierung durchgeführt werden. Bei Beginn der Colitis in den ersten 3 Lebensjahren, bei perianalen Läsionen, einer auffälligen Anamnese für häufige oder ungewöhnliche Infektionen unabhängig vom Alter und Konsanguinität der Eltern sind angeborene Immundefektzustände als Ursache der Colitis durch entsprechende immunologische Untersuchungen auszuschließen. Eine allergische Colitis oder eine Nahrungsmittelallergie als modulierender Faktor der entzündlichen Aktivität ist besonders bei jüngeren Kindern mit Colitis differenzialdiagnostisch zu bedenken.

\section{Histopathologische Diagnostik - Entzündungsdiagnostik}

\section{EMPFEHLUNG 2.22}

Histopathologische Kriterien, die bei der Beurteilung von Biopsien zur Diagnose einer Colitis ulcerosa herangezogen werden sollten, sind:

- diffuse panmukosale chronische Entzündung (Lymphozyten und Plasmazellen) in Kombination mit einer Störung der Kryptenarchitektur/Kryptenatrophie,

- Plasmozytose im basalen Schleimhautstroma

- Panethzell-Metaplasien distal der rechten Kolonflexur

- Reduktion der Zahl von Becherzellen bzw. des Muzingehalts der Einzelzellen, kontinuierliche Verteilung der entzündlichen und strukturellen Schleimhautveränderungen, abnehmender Gradient von distal nach proximal

Evidenzgrad 1, Empfehlungsgrad B, starker Konsens

\section{EMPFEHLUNG 2.23}

Abweichende morphologische Befundmuster können bei der Colitis ulcerosa vorkommen und sollen speziell bei pädiatrischen Patienten berücksichtigt werden. Evidenzgrad 1, Empfehlungsgrad A, Konsens

\section{EMPFEHLUNG 2.24}

Der Pathologiebefund sollte eine Aussage zur histologischen Entzündungsaktivität enthalten.

Expertenkonsens, Empfehlung, Konsens

\section{Hintergrund}

Die Diagnose einer Colitis ulcerosa basiert auf dem Nachweis einer charakteristischen Kombination von klinischen, laborchemischen, endoskopischen, radiologischen und pathomorphologischen Befunden. Einen Goldstandard für die Diagnose gibt es nicht. Auch die pathohistologische Diagnostik beruht auf der synoptischen Beurteilung einer Kombination von Charakteristika, die schwerpunktmäßig die Art und Verteilung der Entzündungsinfiltrate sowie Veränderungen der Mukosaarchitektur betreffen [110 - 123]. Die histologischen Einzelbefunde sind dabei für sich genommen nicht spezifisch und können z. T. auch bei anderen Formen entzündlicher Darmerkrankungen auftreten.

Veränderungen der Mukosaarchitektur bei Colitis ulcerosa beinhalten Irregularitäten in Form, Orientierung und Größe der Krypten (> 10 \% der Krypten; mehr als 2 verzweigte, nicht parallel orientierte Krypten in einer Biopsie) [117, 119, 121, 123]. Der Begriff Kryptenatrophie bezeichnet den Befund einer verminderten Kryptendichte (Distanz von mehr als einem Kryptenquerschnitt zwischen 2 benachbarten Krypten) und/oder einer Distanzbildung zwischen Kryptenbasis und der Lamina muscularis mucosae, zumeist verbunden mit einem basal vermehrten mononukleären, plasmazellreichen Infiltrat [115, 116, 119, 121]. Der Terminus „transmukosale Entzündung“ beinhaltet einen diffus gesteigerten Gehalt an mononukleären Zellen in der Lamina propria unter Einbeziehung der mittleren und basalen Schleimhautabschnitte $[115,119]$. Eine basale Plasmozytose ist definiert als Nachweis von Plasmazellen in den basalen Bezirken (1/5) der Lamina propria oder zwischen der Kryptenbasis und der Lamina muscularis mucosae (subkryptal) [116, 117].

Die bioptische Diagnose einer Colitis ulcerosa in Abgrenzung zum Morbus Crohn und anderen entzündlichen Darmerkrankungen stützt sich insbesondere auf die Beurteilung des Ausmaßes (ausgeprägt, diffus) und der topografischen Verteilung (kontinuierliche Verteilung, abnehmender Gradient von distal nach proximal) der in der Empfehlung 2.22 genannten histopathologischen Kriterien. Daher sollen im Rahmen der Erstdiagnostik Stufenbiopsien aus dem terminalen Ileum und aus allen Kolonabschnitten unter Einbeziehung des Rektums gewonnen werden und die bioptischen Proben bezüglich ihrer Lokalisation gekennzeichnet sein. Die diagnostische Aussagekraft kann so im Vergleich zu singulären bzw. nicht systematischen Biopsien deutlich gesteigert 
werden [111, 112, 124 - 127]. Zum Ausschluss differenzialdiagnostisch relevanter Befunde (z. B. epitheloidzellige Granulome) ist eine Aufarbeitung der Proben in Stufen- oder Serienschnitten zu empfehlen [128, 129].

Unter diesen Voraussetzungen kann die histopathologische Diagnose einer Colitis ulcerosa an Mukosabiopsien mit einer Sensitivität und Spezifität von mehr als $70 \%$ [111 - 113, 118, 123], in einigen Studien von bis über $90 \%$ gestellt werden [120 - 122], zumindest bei aktiver Erkrankung. Einige Autoren propagieren die Beurteilung anhand von standardisierten Score-Schemata mit numerischen Koeffizienten [120 - 122]. Die Diagnose erfordert den Nachweis von zwei bis drei der genannten histomorphologischen Kriterien.

Im Initialstadium der Erkrankung (Krankheitsdauer < 4- 6 Wochen) kann eine Störung der Mukosaarchitektur fehlen; eine spezifische Diagnose ist dann gegebenenfalls nicht möglich. Der Befund einer basalen Plasmozytose ist in diesem Kontext von prädiktiver Relevanz und kann als Frühzeichen einer potenziellen chronisch-entzündlichen Darmerkrankung gewertet werden [116, 117, 130, 131].

In diagnostischer Hinsicht ist zu berücksichtigen, dass das morphologische Erscheinungsbild chronisch-entzündlicher Darmerkrankungen biologischen Variationen unterliegt (phasenhafter Verlauf) und zudem durch die Therapie beeinflusst wird [132, 133]. So kann es im Verlauf einer Colitis ulcerosa durchaus zur diskontinuierlichen Ausprägung des Entzündungsbildes, auch mit Aussparung des Rektums, kommen [134]. Insbesondere bei pädiatrischen CED-Patienten (jünger als 10 Jahre) ist auch ohne vorangegangene Therapie mit abweichenden Mustern zu rechnen [135 - 138]. Informationen zum klinischen Bild (Anamnese, Erkrankungsdauer, Art und Dauer der Therapie, Endoskopiebefund) sind daher für eine effiziente Beurteilung erforderlich und verbessern die Validität der histologischen Befundeinordnung [112]. Dies gilt insbesondere auch im Hinblick auf die differenzialdiagnostische Abgrenzung der Colitis ulcerosa von anderweitigen, ätiologisch oder phänotypisch definierten Entzündungsformen (infektiöse Colitiden, medikamentös induzierte Colitiden, Diversionscolitis, mit Divertikelkrankheit assoziierte Colitis, allergieassoziierte Colitis u. a.) [139-141].

Die histopathologische Einordnung der Entzündungsaktivität bei der Colitis ulcerosa orientiert sich am Ausmaß der Gewebeinfiltration durch segmentkernige neutrophile Granulozyten und der damit assoziierten Schädigung des Darmepithels mit neutrophiler Epithelinvasion, Ausbildung von Kryptitisherden und Kryptenabszessen bis hin zu erosiven und ulzerösen Läsionen [115, 118, 119, 121]. Histologischer Befund und klinische Krankheitsaktivität korrelieren beim individuellen Patienten nur bedingt miteinander [142]. Aus Therapiestudien gibt es allerdings Hinweise auf die Assoziation zwischen klinischer Besserung und Rückgang aktiver histologischer Veränderungen [143]. Ein aktives morphologisches Bild ist mit dem Auftreten rezidivierender Erkrankungsschübe assoziiert [144 - 146]. Eine Aussage zur histologischen Entzündungsaktivität im Pathologiebefund ist daher besonders im Hinblick auf die Verlaufsbeurteilung von Interesse. Zahlreiche Graduierungsschemata sind etabliert (z. B. Riley-Score). Die einzig bisher validierten Scores sind der Nancy histological index und der Robarts histopathology index [147, 148]. Aufgrund der Heterogenität der zahlreichen verfügbaren Scores gibt es jedoch derzeit keine Empfehlung für einen spezifischen histologischen Entzündungsscore. Der potenzielle Nutzen der Histopathologie für die Vorhersage eines Rezidivs und die Beurteilung der adäquaten Kontrolle der Entzündung hat Implikationen für das therapeutische Management und die Verringerung des Neoplasierisikos.

\section{Intraepitheliale Neoplasien (IEN)}

\section{EMPFEHLUNG 2.25}

Die Diagnose von intraepithelialen Neoplasien/Dysplasien bei der Colitis ulcerosa soll nach den jeweils gültigen Kriterien der WHO erfolgen IEN/Dysplasien sollen histopathologisch graduiert werden in niedriggradig, hochgradig oder unklar (indefinite).

Expertenkonsens, starke Empfehlung, starker Konsens

\section{EMPFEHLUNG 2.26}

Bei histologischer Diagnose jeder IEN/Dysplasie sollte der Prozess einer kompetenten (dokumentierten) pathologischen Zweitmeinung im Sinne eines Vier-Augen-Prinzips sichergestellt sein.

Evidenzgrad 3, Empfehlungsgrad B, Konsens

\section{Hintergrund}

Intraepitheliale Neoplasien/Dysplasien werden definiert als eindeutig neoplastische Läsionen des Epitheliums, die auf die Basalmembran begrenzt sind und keine Invasion in die Lamina propria aufweisen [149]. Dysplasien sind der zuverlässigste Marker für ein erhöhtes intestinales Malignomrisiko [150]. Intraepitheliale Neoplasien/Dysplasien werden gemäß dem Grad ihrer neoplastischen Transformation histopathologisch graduiert in niedriggradig, hochgradig oder unklar (indefinite) [149]. Erschwert wird der Nachweis von IEN durch die hohe Untersuchervariabilität der Pathologen [151 - 153] mit einem к-Wert von 0,4 [154], wobei die Variabilität bei niedriggradigen und fraglichen IEN besonders hoch ist. Wegen der erheblichen therapeutischen Konsequenzen sollte jede histopathologische „IEN/Dysplasie“-Diagnose von einem erfahrenen weiteren Pathologen durch eine Zweitbeurteilung bestätigt werden.

\section{EMPFEHLUNG 2.27}

Im Fall einer sichtbaren Läsion mit IEN/Dysplasie sollte eine Differenzierung durch den Endoskopiker der CED-assoziierten Neoplasien in polypoide oder nicht polypoide Läsionen, jeweils mit Angabe des IEN/Dysplasiegrads (LGIEN oder HGIEN), erfolgen, da diese Aussage von therapeutischer Bedeutung ist. Evidenzgrad 3, Empfehlungsgrad B, Konsens 


\section{Hintergrund}

In Übereinstimmung mit den Empfehlungen der ECCO [155, 156] und der internationalen SCENIC-Konsensuskonferenz sollen zukünftig die makroskopischen Beschreibungen von IEN/Dysplasien als DALM (dysplasieassoziierte Läsion oder Masse), ALM (adenomassoziierte Läsion oder Masse), adenomartig, nicht adenomartig und flach nicht mehr verwendet werden, da diese makroskopischen Kriterien oftmals zur Beschreibung verschiedenartig imponierender Läsionen verwendet wurden. Sichtbare Läsionen sollen daher als polypoid oder nicht polypoid klassifiziert werden [157].

Eine polypoide Läsion beinhaltet gestielte (Paris-Klassifikation Typ 1 p) oder sessile (Paris-Klassifikation Typ $1 \mathrm{~s}$ ) Läsionen, die von der Mukosa ausgehend in das Lumen hervorragen (> 2,5 mm) [157]. Diese Läsionen sind normalerweise mittels endoskopischer Resektion entfernbar [158].

Nicht polypoide Läsionen beschreiben Läsionen der Paris-Klassifikation Typ Ila (flat-elevated), Paris-Klassifikation Typ Ilb (flatflat) und Paris-Klassifikation Typ Ilc (flat-depressed). Weiterhin umfassen diese samtartige Flecken, Plaques, irreguläre Unebenheiten und Knötchen, Verdickungen, strikturierende Läsionen und breitbasierte Massen. Diese Läsionen sind nicht immer durch eine endoskopische Resektion entfernbar [159-162]. Die Differenzierung der Läsion erfolgt aufgrund ihrer endoskopischen Erscheinung. Eine endoskopisch unsichtbare dysplastische Läsion bezeichnet eine histologisch festgestellte IEN/Dysplasie, die während der Koloskopie nicht sichtbar war.

\section{Überwachungskoloskopie}

\section{EMPFEHLUNG 2.28}

Da die colitisassoziierte Kolonkarzinommortalität durch eine endoskopische Überwachung gesenkt werden kann, sollten angepasst an eine Risikostratifizierung Überwachungskoloskopien erfolgen.

Evidenzgrad 3, Empfehlungsgrad B, Konsens

\section{Hintergrund}

Die Effektivität der Überwachungskoloskopie-Programme ist bis heute nicht in randomisiert kontrollierten Studien untersucht worden. Es gibt aber eine große Zahl von Untersuchungen als FallKontroll-Studien und auch als Fallserien, die für eine Effektivität der Vorsorgekoloskopie bei der Colitis ulcerosa sprechen [163167]. Drei Fall-Kontroll-Studien untersuchten die Effektivität der Überwachungskoloskopie bei der Colitis ulcerosa [168 - 170]. Diese drei Studien und zwei weitere Studien wurden kürzlich in einer Metaanalyse zusammengefaßt [171]. Hierbei ergibt sich eine verminderte Mortalität durch eine Überwachungskoloskopie bei Colitis ulcerosa [171]. Die Evidenzqualität der zugrunde liegenden Studien wird jedoch als gering eingestuft.

\section{EMPFEHLUNG 2.29}

Zur Festlegung der Überwachungsstrategie sollte bei allen CU-Patienten unabhängig von der Krankheitsaktivität eine Kontrollkoloskopie mit Entnahme von zumindest zwei Biopsien aus jedem Kolonsegment, zusätzlich zu gezielten Biopsien zur Erfassung des Befallsmusters, 6 - 8 Jahre nach Beginn der Symptomatik/Diagnosestellung erfolgen.

Evidenzgrad 2, Empfehlungsgrad B, starker Konsens

\section{Hintergrund}

Gegenüber der früheren Auffassung, dass kolorektale Karzinome relativ selten vor einer Krankheitsdauer von acht Jahren auftreten, gibt es auch Untersuchungen, die zeigen, dass ein signifikanter Anteil von kolorektalen Karzinomen auch schon vor dem 8. Krankheitsjahr auftreten kann [172, 173]. Eine holländische Studie konnte zeigen, dass $22 \%$ der CU-Patienten, die ein Karzinom entwickelten, dies schon vor dem Beginn der regulären Überwachungskoloskopien zeigten [174]. Aus diesen Daten folgt die Empfehlung, zwischen dem 6. und dem 8. Erkrankungsjahr eine Kontrollkoloskopie unabhängig von der Krankheitsaktivität durchzuführen, um die Krankheitsausdehnung auch histologisch zu erheben und so das Überwachungsprogramm im Hinblick auf den zeitlichen Ablauf festlegen zu können.

\section{EMPFEHLUNG 2.30}

Wenn die Krankheitsaktivität auf das Rektum ohne Nachweis einer vorherigen oder aktuellen endoskopischen und/oder mikroskopischen Entzündung proximal zum Rektum beschränkt ist, sollte die Einbeziehung in ein regelmäßiges Überwachungskoloskopie-Programm nicht erfolgen.

Expertenkonsens, Empfehlung, starker Konsens

Zur Kontrolle und um eine Ausdehnung der Colitis ulcerosa nicht zu übersehen, kann alle 5 Jahre eine Koloskopiekontrolle sinnvoll sein.

Expertenkonsens, Empfehlung offen, starker Konsens

\section{EMPFEHLUNG 2.31}

Bei Patienten mit Befall über das Rektum hinaus sollen regelmäßige Überwachungskoloskopien ab dem 8. Erkrankungsjahr durchgeführt werden.

Expertenkonsens, starke Empfehlung, Konsens

Die Überwachungsstrategie sollte individuell abgestimmt werden, und dabei sollte sich das Intervall nach einer Risikostratifizierung richten. Nach dieser Risikostratifizierung sollte bei Patienten mit einem hohen Risiko (Stenose, IEN innerhalb der letzten 5 Jahre, ausgedehnte Colitis mit ausgeprägter Entzündung oder erstgradigem Verwandten mit KRK < 50 J.) jährlich, bei Patienten mit einem intermediären Risiko (Colitis mit milder oder mäßiger Entzündung, viele Pseudopolypen, erstgradiger Verwandter mit KRK $\geq 50$ J.) alle 2 - 3 Jahre und bei 
- Tab. 6 Untersuchungsintervall der Überwachungskoloskopie nach Risikostratifizierung.

Untersuchungsintervall ab dem 8. Erkrankungsjahr zur Überwachungskoloskopie nach Risikostratifizierung bei Colitis ulcerosa (bei Erfüllung eines Kriteriums gilt das jeweils höchste Risiko) nach Statement 2.31 und 2.32

jedes Jahr (hohes Risiko)

ausgedehnte Colitis mit hochgradiger Entzündung

erstgradiger Verwandter mit KRK $<50$ Jahre

IEN in den letzten 5 Jahren

PSC (jährlich ab Diagnosestellung)

Stenose

\section{alle 2 - 3 Jahre (intermediäres Risiko)}

Colitis mit milder bis mäßiggradiger Colitis alle 4 Jahre (geringes Risiko)

Es liegt keines der Kriterien für ein hohes oder intermediäres Risiko vor.
Patienten mit einem niedrigen Risiko (es liegen keine der genannten Faktoren vor) alle 4 Jahre eine Überwachungskoloskopie durchgeführt werden.

Expertenkonsens, Empfehlung, Konsens

\section{Hintergrund}

Das Kolonkarzinomrisiko ist bei Patienten mit einer Colitis ulcerosa im Vergleich zur Normalbevölkerung erhöht. Dabei hängt das Risiko von der Ausdehnung der Erkrankung ab. Nach verschiedenen Untersuchungen ist das Risiko deutlich erhöht bei einer ausgedehnten Colitis und immer noch relevant erhöht bei der Linksseitencolitis, aber nicht eindeutig erhöht bei der Proktitis ulcerosa [175].

Patienten mit Colitis ulcerosa zeigen in zahlreichen Studien ein erhöhtes Risiko für ein kolorektales Karzinom, wobei unterschiedliche Angaben über die Höhe des Risikos zu finden sind. Während frühere Studien aus Zentren - zusammengefasst in einer Metaanalyse - deutlich höhere Zahlen (KRK-Risiko bei CU-Patienten nach 10 Jahren $2 \%$, nach 20 J. $8 \%$ und nach 30 J. $18 \%$ ) aufwiesen [175], zeigten populationsbasierte Studien der letzten Jahre nur gering erhöhte Karzinomraten (KRK-Risiko nach 10 Jahren 0,61,2\%, nach 20 Jahren 2,1 - 5,4\% und nach 30 Jahren 4,7 - 7,5\%) [176, 177]. Lediglich in Dänemark fand sich keine erhöhte Inzidenz für das KRK bei den CU-Patienten, möglicherweise wegen einer höheren Kolektomierate in Dänemark in den Jahren der Untersuchung [172]. Die Häufigkeit korreliert mit der Erkrankungsdauer, der Ausdehnung und der entzündlichen Aktivität/ Pseudopolypen [3, 175, 178-181].

Im Jahr 2001 publizierte Eaden eine Metaanalyse aus 116 Studien und berechnete daraus ein kumulatives Risiko für ein Colitiskarzinom nach 30 Jahren mit 18\% [175]. In einer weiteren Metaanalyse wurde ein 2,4-fach erhöhtes Risiko für ein rektales Karzinom bei CU-Patienten beschrieben [182]. In weiteren Arbeiten finden sich aber jetzt im Verlauf Hinweise, dass das kolorektale Karzinomrisiko eher abzunehmen scheint. Jess fand in ihrer Arbeit von 2013 aus Dänemark weiter kein erhöhtes KRK-Risiko für CUPatienten mit einem Faktor von 1,12 $(0,97$ - 1,28) und Choi beschrieb 2015 in seiner Fallserie ein nur geringer erhöhtes KRK-Risiko (10 J.: 0,1\%, 20 J.: 2,9\% und nach 30 J. 6,7\%) [183, 184].
Eine neuere australische Untersuchung [185] beschrieb ein kumulatives kolorektales Karzinomrisiko bei Colitis ulcerosa von $1 \%$ bei 10 Jahren, 3 \% bei 20 Jahren und $7 \%$ bei 30 Jahren. Möglicherweise spiegeln sich darin die ansteigenden Implementierungen der Überwachungsprogramme wider, ebenso wie eine effektivere entzündungskontrollierende medikamentöse Therapie [186].

Überwachungskoloskopien zielen auf die Detektion von Neoplasien mit hoher Sensitivität und Spezifität ab. Dazu gehört, dass der Darm keine wesentliche Entzündung aufweist, die als intraepitheliale Neoplasien histologisch fehlgedeutet werden könnte. In Analogie zu Vorsorgekoloskopien in der Normalbevölkerung ist davon auszugehen, dass die Qualität der Koloskopievorbereitung die Detektionsrate von Läsionen deutlich erhöht [187]. Ebenso besteht eine Korrelation zwischen der Rückzugszeit und der Detektionsrate von Neoplasien [188]. Als Neuerung in dieser Leitlinie soll das Überwachungsintervall der Vorsorgekoloskopien an eine Risikostratifizierung angepasst werden. Dafür wurden verschiedene evidenzbasierte Risikokonstellationen definiert ( Tab.6). Angepasst an diese Risikokonstellationen liegt das Überwachungsintervall danach bei 1 Jahr, 2 - 3 Jahren oder 4 Jahren, je nach beschriebenen Risikokonstellationen [4, 152, 159, 178, 180, 186, 189 - 198]. In manchen Studien wird auch berichtet, dass als zusätzliche Risikofaktoren ein Kolonkarzinom in der Familienanamnese, das Vorhandensein einer Backwash-Ileitis und eine Colitis-Erstmanifestation im Kindes- und Jugendalter zum Tragen kommen [175, 181, 199]. Die Datenlage diesbezüglich ist jedoch uneinheitlich.

\section{EMPFEHLUNG 2.32}

Wenn gleichzeitig eine PSC besteht, sollten die Überwachungskoloskopien unabhängig von der Krankheitsaktivität und der Ausdehnung der CU ab dem Zeitpunkt der PSCDiagnosestellung jährlich erfolgen.

Evidenzlevel 3, Empfehlungsgrad B, Konsens

\section{Hintergrund}

Eine besondere Risikogruppe sind Patienten, die gleichzeitig eine primär sklerosierende Cholangitis (PSC) haben. Eine Metaana- 
lyse ergab ein fünffach erhöhtes Karzinomrisiko [4, 191]. Deshalb sollten diese Patienten auch schon ab der Diagnosestellung jährlich in ein Überwachungskoloskopie-Programm einbezogen werden. In verschiedenen weiteren Untersuchungen zeigte sich, dass das Risiko eines kolorektalen Karzinoms bei CU-Patienten mit PSC nicht nur 5-mal höher [4] ist, sondern dass es auch früher auftritt [152] und häufiger im rechtsseitigen Kolon zu finden ist [191].

\section{EMPFEHLUNG 2.33}

Die Überwachungskoloskopie mit Biopsieentnahme sollte möglichst in der Remissionsphase durchgeführt werden, da die histomorphologische Abgrenzung von entzündlichen gegenüber neoplastischen Veränderungen sonst schwierig sein kann.

Evidenzgrad 4, Empfehlungsgrad B, Konsens

\section{Hintergrund}

Befindet sich das Colon nicht in einem weitgehend entzündungsfreien Zustand, können entzündliche Veränderungen Ähnlichkeiten zu IEN haben. Da es für den Pathologen schwierig sein kann, in entzündlichen Arealen eine IEN sicher zu beurteilen, kann es bei einer vom Pathologen als fragliche IEN eingestuften Läsion Sinn machen, innerhalb von 3 Monaten (ggf. nach einer Intensivierung der antiinflammatorischen Therapie) eine endoskopische Kontrolle durchzuführen.

\section{EMPFEHLUNG 2.34}

Gezielte Biopsien sollten aus allen endoskopisch suspekten Läsionen entnommen werden. Die Überwachungskoloskopie sollte in einem sauberen Darm mit ausreichender Rückzugszeit erfolgen.

Evidenzgrad 1, Empfehlungsgrad B, Konsens

\section{EMPFEHLUNG 2.35}

Die Überwachungskoloskopie sollte als Chromoendoskopie mit gezielten Biopsien ohne zusätzliche zufällige Biopsien als Überwachungsverfahren der Wahl durchgeführt werden. Alternativ kann eine hochauflösende Weißlicht-Endoskopie (HDWLE) mit gezielten Biopsien jeder sichtbaren Läsion ohne zusätzliche zufällige Biopsien mit besonderer Sorgfalt und entsprechender Rückzugszeit durchgeführt werden.

Evidenzgrad 1, Empfehlungsgrad B, Konsens

Falls die HDWLE Endoskopie nicht zur Verfügung steht, sollen zusätzlich ungezielte Stufenbiopsien entnommen werden. Evidenzgrad 2, Empfehlungsgrad A, Konsens

\section{Hintergrund}

Zur Durchführung der Überwachungskoloskopie gibt es seit vielen Jahren Diskussionen um die Rahmenbedingungen bezüg- lich zusätzlicher Zufallsbiopsien und/oder einer Durchführung einer Chromoendoskopie. Die Chromoendoskopie ist bei der Durchführung der Überwachungskoloskopie etabliert und zeigt gute Ergebnisse auch ohne die zusätzlichen Zufallsbiopsien. Darum wird in der Empfehlung auch die Chromoendoskopie mit gezielter Biopsie ohne Zufallsbiopsien als Überwachungsverfahren der Wahl angeführt. Dazu gibt es zahlreiche Studien [200 206]. Vergleiche der Chromoendoskopie zur Dysplasiedetektion mit hoch auflösenden Endoskopen ohne Chromoendoskopie finden sich aber erst in den neueren Untersuchungen [206 - 210]. Die Entwicklung in den letzten Jahren mit „High-Resolution“-Endoskopen hat die Detektionsrate von Dysplasien deutlich gesteigert [211].

Eine kürzlich publizierte Studie aus Japan [209] konnte zeigen, dass alleinige gezielte Biopsien bei der Durchführung einer „HighResolution“-Koloskopie der zusätzlichen Durchführung von Zufallsbiopsien nicht unterlegen sind. Es gibt weitere Untersuchungen zur Dysplasiediagnostik, die zeigen, dass die Zufallsbiopsien bei einem Setting mit einem hochauflösenden Koloskopiesystem keinen höheren Gewinn als die Untersuchungen mit nur gezielten Biopsien erbringen. In einem systematischen Review von randomisierten Studien aus Italien [207] fanden sich bei der gemeinsamen Betrachtung von drei Studien mit 190 Patienten keine Unterschiede bezüglich der Dysplasie-Erkennungsraten bei der Chromoendoskopie versus „High-Resolution“-Weißlichtendoskopie (HDWLE). Der Unterschied bezüglich der Dysplasiefindung war allerdings bei dem Vergleich zwischen Chromoendoskopie und nicht hochauflösender Weißlichtendoskopie (WLE) signifikant. Zu einem ähnlichen Ergebnis kommt eine unizentrische Studie aus Kanada [208], die 454 IBD-Patienten in einem Surveillance-Programm untersuchte. Danach war das Ergebnis zur Erkennung von Dysplasien bei gezielten Biopsien besser als bei Zufallsbiopsien in der HDWLE und in der Chromoendoskopie. Dieses Ergebnis fand sich aber nicht bei der normalen Weißlichtendoskopie (WLE). Eine randomisierte Multicenterstudie aus Japan bei 246 CU-Patienten verglich die gefundenen Dysplasieraten bei den Zufallsbiopsien und bei gezielten Biopsien, ganz überwiegend bei durchgeführten „High-Resolution“-Endoskopien (HDWLE). In beiden Gruppen, Zufallsbiopsien und gezielte Biopsien, fanden sich gleich viele intraepitheliale Neoplasien (IEN)/Dysplasien. Diese neuen Studienergebnisse bei der HDWLE begründen auch die alternative Aufnahme der HDWLE mit gezielten Biopsien ohne Zufallsbiopsien als mögliche Option bei der Überwachungskoloskopie [206-210, 212].

\section{EMPFEHLUNG 2.36}

Der Stellenwert der hochauflösenden virtuellen Chromoendoskopie (NBI, FICE, iScan) in Kombination mit gezielten Biopsien ohne zufällige Biopsien ist nicht ausreichend definiert, und sie sollte deshalb nicht als alleinige Strategie verfolgt werden.

Evidenzgrad 1, Empfehlungsgrad B, starker Konsens 


\section{Hintergrund}

Der Stellenwert der virtuellen Chromoendoskopie (NBI, FICE, iScan) als zusätzliches Tool für die Verbesserung der Effektivität der Überwachungskoloskopie bei der Colitis ulcerosa ist aufgrund der vorliegenden Studien nicht klar definiert, und sie sollte deshalb nicht als alleinige Strategie verfolgt werden [213-220].

\section{EMPFEHLUNG 2.37}

Bei Vorliegen einer fraglichen IEN/Dysplasie sollte eine endoskopische Kontrolle ggf. nach Intensivierung der antiinflammatorischen Therapie innerhalb von 3-6 Monaten durchgeführt werden.

Evidenzgrad 2, Empfehlungsgrad B, Konsens

\section{EMPFEHLUNG 2.38}

Bei dem Nachweis einer endoskopisch nicht resektablen Läsion mit IEN/Dysplasie oder eines Adenokarzinoms soll wegen der hohen Assoziation mit einem metachronen oder synchronen Karzinom eine Proktokolektomie erfolgen. Evidenzgrad 2, Empfehlungsgrad A, starker Konsens

\section{EMPFEHLUNG 2.39}

Bei endoskopisch komplett resezierten polypoiden Läsionen mit Dysplasie/IEN ohne weitere Dysplasien im übrigen Kolon kann als Überwachungsstrategie die Koloskopie in jährlichen Abständen empfohlen werden.

Expertenkonsens, Empfehlung offen, Konsens

\section{EMPFEHLUNG 2.40}

Nach der kompletten endoskopischen Resektion von nicht polypoiden Läsionen mit Dysplasie/IEN ohne weitere Dysplasien im übrigen Kolon sollte die endoskopische Überwachung in jährlichen Abständen durchgeführt werden.

Expertenkonsens, Empfehlung, Konsens

\section{EMPFEHLUNG 2.41}

Falls durch Zweitbefundung histologisch bestätigte IEN/Dysplasien aus endoskopisch unauffälligen Arealen detektiert werden, sollte eine erneute endoskopische Abklärung primär durch Chromoendoskopie mit hochauflösender Weißlichtendoskopie (HDWLE) durch einen in der Überwachungskoloskopie erfahrenen Untersucher erfolgen.

Expertenkonsens, Empfehlung, Konsens

\section{EMPFEHLUNG 2.42}

Falls IEN/Dysplasien aus endoskopisch unauffälligen Arealen detektiert werden, sollte in Abhängigkeit von der Graduierung der Dysplasie eine endoskopische und bioptische Kontrolle durchgeführt werden. Bei bestätigten niedriggradigen IEN sollte eine erneute endoskopische/bioptische Kontrolle innerhalb von 3-6 Monaten durchgeführt werden.

Expertenkonsens, Empfehlung, Konsens

Alternativ kann auch eine Proktokolektomie mit dem Patienten diskutiert werden. Bei bestätigten hochgradigen IEN soll eine Empfehlung zur Proktokolektomie gegeben werden.

Expertenkonsens, offene Empfehlung, Konsens

\section{EMPFEHLUNG 2.43}

Polypen mit Dysplasien, die sich proximal zu den Segmenten mit anamnestisch maximaler makroskopischer oder histologischer CU Beteiligung ergeben, werden als sporadische Adenome betrachtet und sollten bei endoskopischer Resektabilität entsprechend behandelt werden.

Expertenkonsens, Empfehlung, Konsens

\section{Hintergrund}

Der Nachweis einer intraepithelialen Neoplasie (IEN) sowie deren Gradeinteilung ist für die Beurteilung des Kolonkarzinomrisikos bei Patienten mit CU von entscheidender Bedeutung. Anhand von Kolektomiepräparaten mit nachgewiesenem Karzinom fanden sich in postoperativ ungezielt entnommenen Quadrantenbiopsien IEN bei $74 \%$ der Fälle, und gleichzeitig enthielten Kolektomiepräparate ohne Karzinom in $26 \%$ der Fälle IEN in ungezielten Quadrantenbiopsien [221]. Eine Metaanalyse zeigte, dass sogar bei niedriggradigen IEN das Karzinomrisiko 9-fach erhöht ist [197].

Kolonkarzinome sind bei Patienten mit Colitis ulcerosa und Nachweis von IEN gehäuft. Grundsätzlich ist zwischen flachen und polypoiden Läsionen zu unterscheiden. Befindet sich das Kolon nicht in einem weitgehend entzündungsfreien Zustand, können entzündliche Veränderungen Ähnlichkeiten zu IEN haben. Diese werden dann i. d. R. als fragliche IEN bezeichnet, und eine Intensivierung der antiinflammatorischen Therapie mit kurzfristiger Kontrolle erscheint sinnvoll. Zeigt sich dagegen eine flache, hochgradige IEN in endoskopisch unauffälligen Arealen, liegt das Risiko, dass gleichzeitig ein Karzinom besteht, zwischen 42 und $45 \%$ und es sollte deshalb eine Proktokolektomie durchgeführt werden $[180,193]$.

Aus flachen, niedriggradigen IEN können einerseits hochgradige IEN oder Karzinome hervorgehen, andererseits können niedriggradige IEN ein Indikator für synchrone Karzinome sein. Eine Metaanalyse errechnete ein 9-fach erhöhtes Risiko für die Entwicklung eines Karzinoms, wenn eine niedriggradige IEN nachgewiesen wird. Andererseits zeigen einzelne Studien, dass das Risiko für die Entwicklung eines colitisassoziierten KRK bei Patienten mit niedriggradigen IEN nicht oder nicht signifikant erhöht ist [194, 
197, 198]. Eine populationsbasierte Studie konnte auch keinen vermehrten Übergang zu Karzinomen finden [196].

Der Nachweis einer IEN unabhängig vom Grad, korreliert mit einer Sensitivität und Spezifität von 74\% mit einer KRK-Entstehung, während in der gleichen Studie eine hochgradige IEN zwar eine geringere Sensitivität (34\%), aber mit 98\% eine höhere Spezifität für einen KRK-Nachweis zeigte [221]. In einer Metaanalyse war eine niedriggradige IEN mit einem 9-fach erhöhten KRK-Risiko und mit einem 12-fach erhöhten Risiko für eine fortgeschrittene Neoplasie korreliert [197]. In einer kürzlich veröffentlichten systematischen Metaanalyse von Kohortenstudien war eine niedriggradige IEN mit einem jährlichen Risiko der Inzidenz eines KRK von $0,8 \%$ und einer fortgeschrittenen Neoplasie von 1,8\% assoziiert. Faktoren, die signifikant mit einer Progression der Dysplasie assoziiert waren, umfassten das Vorliegen einer PSC (OR 3,4), eine nicht sichtbare Dysplasie (OR 1,9), eine distale Lokalisation (OR 2,0) und multifokale Dysplasien (OR 3,5). Weiterhin wurde bei $17 \%$ der bei niedriggradiger IEN kolektomierten Patienten ein synchrones KRK nachgewiesen [222]. Einzelstudien, in denen kein erhöhtes Malignomrisiko bei niedriggradiger IEN gefunden wurde, sollten im Kontext der Metaanalysen betrachtet werden [196]. Die Diagnose einer niedriggradigen IEN ist daher mit einem substantiellen Karzinomrisiko assoziiert und hat erhebliche prognostische Implikationen [194, 198, 223].

Die Datenlage ist somit bei den niedriggradigen IEN kontrovers und eine engmaschige Überwachung mit bioptischer Kontrolle nach 3-6 Monaten wird als Alternative zur Kolektomie für vertretbar gehalten.

Erhabene Läsionen mit IEN wurden ursprünglich als dysplasieassoziierte Läsionen oder Massen (DALM) klassifiziert [160]. Das Risiko eines Kolonkarzinoms beim Vorliegen einer DALM wurde als sehr hoch eingeschätzt [193]. Die Einordnung als DALM ist aber nicht ganz einfach und wurde uneinheitlich vorgenommen. Wegen der schwierigen und uneinheitlichen Zuordnung wurde der Begriff DALM schon bei der ECCO-UC-Leitlinie 2012 verlassen und durch den Begriff „Raised Lesions with Dysplasia (RLD)“ ersetzt [189].

Solche Läsionen können auch sporadischen Adenomen ähneln und dann endoskopisch reseziert werden. Wenn die Resektion histologisch komplett ist, in der Umgebung keine IEN zu finden sind und wenn sich im Restkolon keine IEN findet, kann auf eine Kolektomie verzichtet werden. Allerdings sollten diese Patienten kurzfristig kontrolliert werden, weil sie zur Entwicklung erhabener Läsionen neigen (RLD) [224-226]. In einer Metaanalyse, in die 10 Studien mit 376 Patienten eingeschlossen wurden, zeigte sich nach Resektion der Polypen ein geringes Risiko für die Entwicklung eines kolorektalen Karzinoms. Da diese Patienten jedoch ein etwa 10-fach erhöhtes Risiko für die Entwicklung weiterer Dysplasien haben, sind engmaschige Verlaufskontrollen auch nach endoskopischer Resektion indiziert [227].

Bei dem Nachweis einer nicht resektablen Läsion mit IEN/ Dysplasie oder bei einem Adenokarzinom sollte wegen der hohen Assoziation mit einem metachronen oder synchronen Karzinom eine Proktokolektomie erfolgen. Bei endoskopisch komplett resezierbaren polypoiden Läsionen mit Dysplasie/IEN ohne weitere Dysplasien im übrigen Kolon sollte als Überwachungsstrategie die Koloskopie in jährlichen Abständen empfohlen werden [157].
Das gleiche Vorgehen gilt auch bei einer kompletten Resektion von nicht polypoiden Läsionen mit Dysplasie/IEN. Falls sich als Zufallsbefund aus endoskopisch unauffälligen Arealen eine durch Zweitbefundung histologisch bestätigte IEN/Dysplasie zeigt, sollte eine erneute endoskopische Abklärung primär durch eine Chromoendoskopie mit hoch auflösender Weißlichtendoskopie (HDWLE) durch einen in der Überwachungskoloskopie erfahrenen Untersucher erfolgen.

Finden sich aus endoskopisch unauffälligen Arealen bestätigte niedriggradige intraepitheliale Neoplasien, sollte erneut eine endoskopisch-bioptische Kontrolle innerhalb von 3-6 Monaten durchgeführt werden. Alternativ kann auch bei diesen Patienten eine Proktokolektomie diskutiert werden. Bei einer bestätigten hochgradigen intraepithelialen Neoplasie aus endoskopisch unauffälligen Arealen soll eine Empfehlung zur Proktokolektomie gegeben werden [157].

Polypen mit Dysplasien, die sich proximal zu den Segmenten mit Colitisbefall ergeben, werden als sporadische Adenome betrachtet und sollten bei endoskopischer Resektabilität entsprechend abgetragen werden.

\section{EMPFEHLUNG 2.44}

Beim zusätzlichen Nachweis einer PSC kann zur Prophylaxe eines colitisassoziierten Karzinoms Ursodesoxycholsäure eingesetzt werden.

Evidenzgrad 2, Empfehlungsgrad 0, Konsens

\section{Hintergrund}

Eine Langzeittherapie mit 5-ASA sollte den CU-Patienten unter dem Aspekt der Karzinomprävention angeboten werden (siehe dazu auch 3.18). Bei zusätzlichem PSC-Nachweis konnte in einer Follow-up-Studie einer prospektiven, plazebokontrollierten Studie (PSC-UDCA-Studie) gezeigt werden, dass Ursodesoxycholsäure das Kolonkarzinomrisiko bei Patienten mit PSC und Colitis ulcerosa um $74 \%$ vermindert [228]. Eine Querschnittsuntersuchung aus einer prospektiven Überwachungsstudie hatte zuvor eine deutliche Risikoreduktion gezeigt [229]. Einschränkend ist zu erwähnen, dass eine prospektiv-randomisierte Studie aus dem Jahr 2009 insgesamt eine leicht erhöhte Mortalität bei Patienten mit PSC unter einer Ursodesoxycholsäure-Hochdosistherapie (28 $30 \mathrm{mg} / \mathrm{kg} \mathrm{KG} / \mathrm{Tag}$ ) gefunden hat [230]. Vor diesem Hintergrund wird eine Behandlung mit 13-20 mg/kg KG pro Tag empfohlen.

\section{Behandlung der aktiven Erkrankung und remissionserhaltende Therapie}

\section{Allgemeine Therapieziele}

Der akute Schub einer Colitis ulcerosa ist gekennzeichnet durch typische Beschwerden (blutige Diarrhoen, Tenesmen, imperativer Stuhldrang). Für die Therapieentscheidung spielen neben der Intensität der Symptome das endoskopische Befallsmuster (Proktitis, Linksseitencolitis, Pancolitis), der Krankheitsverlauf, 
das Ansprechen auf die vorangehende Therapie, die Krankheitsdauer, extraintestinale Manifestationen wie auch Begleiterkrankungen und Patientenpräferenzen eine wichtige Rolle. Die möglichen Therapieoptionen sollten mit dem Patienten individuell abgestimmt werden. So sind insbesondere bei der Abwägung zwischen ambulanter und stationärer Therapie die Möglichkeiten von Arzt und Patienten besonders zu beachten.

\section{STATEMENT 3.0}

Das primäre Ziel der Colitis ulcerosa-Therapie sind das rasche Erreichen einer klinischen Remission und die Bewahrung einer langfristigen steroidfreien klinischen und endoskopischen Remission.

Expertenkonsens, starker Konsens

\section{EMPFEHLUNG 3.1}

Vor Einleitung einer antientzündlichen Therapie sollte eine Entzündungsaktivität objektiv nachgewiesen werden.

Expertenkonsens, Empfehlung, starker Konsens

\section{Hintergrund}

Alle verfügbaren antientzündlichen Therapien zielen auf die Beeinflussung entzündlicher Aktivität ab, diese stellt somit die Conditio sine qua non in der pharmakologischen Therapie der Colitis ulcerosa dar. Das Ausmaß der seitens der Patienten beklagten Beschwerden stimmt nicht selten ungenügend mit dem Ausmaß der objektiv mittels Biomarker, Endoskopie oder Sonografie überprüfbaren Inflammation überein, insbesondere bei langem Krankheitsverlauf, bei dem eine Überlappung zum Reizdarmsyndrom beschrieben ist. Eine erhöhte klinische Aktivität ist somit nicht unbedingt durch Entzündung verursacht.

\section{EMPFEHLUNG 3.2}

Bei allen Patienten sollte nach erfolgreicher Schubtherapie eine langfristige Remissionserhaltungstherapie durchgeführt werden.

Expertenkonsens, Empfehlung, starker Konsens

\section{Hintergrund}

Betrachtet man die Remissionsraten in Plazebogruppen umfangreicher Studien, die den spontanen Verlauf der Colitis ulcerosa widerspiegeln, so erfahren die meisten Patienten nach einem Schub innerhalb von 12 Monaten einen erneuten Schub, sodass die Remissionsraten nach 12 Monaten oftmals < 50\% liegen. Eine dauerhafte Therapie mit Aminosalizylaten, Thiopurinen oder Biologika erhöht die Raten anhaltender Remission [231-233]. Tritt eine tiefe klinische und endoskopische Remission ein, erhöht sich die Wahrscheinlichkeit für eine länger anhaltende Remission, wie nachträgliche Analysen der ACT-1- und ACT-2-Studiendaten bei Patienten mit Colitis ulcerosa unter Infliximab-Therapie zeigen konnten [78, 234]. Es konnte ein positiver Zusammenhang zwischen tiefer Remission in Form einer Mukosaheilung und dem klinischen Verlauf gezeigt werden [78, 79]. Nichtsdestotrotz bleibt weiterhin unklar, ob eine medikamentös induzierte Mukosaheilung im Sinne einer „Treat-to-target“-Strategie den weiteren Verlauf beeinflusst oder ob die Patienten, die eine Mukosaheilung erfahren, nicht eine Subgruppe mit milderem Verlauf darstellen.

\section{STATEMENT 3.3}

Die Wahl und die Dauer der geeigneten Schub- und Erhaltungstherapie hängen von der Erkrankungsausbreitung, dem Erkrankungsverlauf (Häufigkeit und Schweregrad der Erkrankungsschübe), Ansprechen auf und Nebenwirkungen durch vorangegangene Therapien, Schweregrad des letzten Erkrankungsschubs, der remissionsinduzierenden Medikation, der Sicherheit der remissionserhaltenden Therapie und dem Potenzial einer Dysplasie- und Krebsprävention ab.

Expertenkonsens, starker Konsens

\section{EMPFEHLUNG 3.4}

Die medikamentösen Therapiemöglichkeiten und -risiken sollten gegen eine operative Therapie abgewogen werden. Expertenkonsens, Empfehlung, starker Konsens

\section{Therapie der unkomplizierten Colitis ulcerosa}

Proktitis

\section{EMPFEHLUNG 3.5}

Eine leichte bis mäßig aktive Proktitis soll zunächst mit Mesalazin $\geq 1000 \mathrm{mg} / \mathrm{d}$ als Suppositorium einmal täglich behandelt werden.

Evidenzgrad 1, Empfehlungsgrad A, starker Konsens

Mesalazinschaum und Mesalazineinläufe stellen eine äquivalente therapeutische Alternative dar.

Evidenzgrad 2, starker Konsens

\section{Hintergrund}

Bei einer auf das Rektum beschränkten Aktivität der Colitis ulcerosa ist eine topische Therapie mit 5-ASA indiziert, deren Wirksamkeit im Hinblick auf symptomatisches, endoskopisches und histologisches Ansprechen durch zahlreiche Studien belegt ist [235 - 237]. Die Dosis sollte dabei mindestens $1 \mathrm{~g} /$ Tag betragen; ein Vorteil für höhere Dosen ist nicht belegt [238]. Dabei sollen vorzugsweise Suppositorien eingesetzt werden, da sie eine günstigere Freisetzung aufweisen und seitens der Patienten besser toleriert werden [239]. Die zusätzliche orale Gabe von 5-ASA kann erwogen werden [240], wobei keine Studien mit dem spezifischen Befallsmuster der Proktitis vorhanden sind. In einer einzel- 
nen Studie zur Therapie der Proktitis war die rektale Gabe von 5-ASA der oralen Therapie überlegen [241].

\section{EMPFEHLUNG 3.6}

Bei Versagen der Monotherapie sollte die rektale Mesalazinanwendung entweder mit topischen Steroiden oder mit einer oralen Gabe von Mesalazin freisetzenden Präparaten kombiniert werden.

Evidenzgrad 1, Empfehlungsgrad B, starker Konsens

\section{Hintergrund}

Bei fehlendem Ansprechen sollte eine Kombination mit topischen Steroiden erfolgen, wobei zwei Metaanalysen zeigen konnten, dass rektale 5-ASA-Präparationen effektiver sind als topische Steroide [242, 243]. Die Gabe von Beclomethasondipropionat $(3 \mathrm{mg})$ in Kombination mit $2 \mathrm{~g}$ 5-ASA-Einläufen war in einer Studie den jeweils einzelnen Therapien überlegen [244]. Ebenso hat eine randomisierte Studie zeigen können, dass $2 \mathrm{~g}$ Budesonid-Rektalschaum bei leicht- bis mäßiggradiger Proktosigmoiditis effektiver sind als Plazebo [245], sowohl mit als auch ohne Kombination mit einem 5-ASA-Präparat [246]. Ein direkter Vergleich zu 5-ASA liegt jedoch nicht vor. Für dieses Befallsmuster scheint die Einnahme eines 5-ASA-Granulats günstig [247].

Erzielen die o.g. Therapien nicht den gewünschten Erfolg, sollten zunächst die Therapieadhärenz sowie die endoskopische Ausprägung bestätigt werden und ggf. die Therapieprinzipien der schweren Colitis ulcerosa mit beliebiger Ausdehnung angewendet werden. Zu diesen Therapieprinzipien ergänzend war in kleinen Studien die rektale Gabe von Tacrolimus erfolgreich [248, 249]. Eine solche Therapie sollte jedoch in entsprechend erfahrenen Zentren durchgeführt werden.

\section{Linksseitencolitis}

\section{EMPFEHLUNG 3.7}

Eine leichte bis mäßig schwere linksseitige CU sollte initial mit rektalem Mesalazin in Form von Einläufen oder Schäumen $(\geq 1 \mathrm{~g} / \mathrm{d})$ in Kombination mit oralen, Mesalazin freisetzenden Präparaten ( $\geq 3 \mathrm{~g} / \mathrm{d}$ ) behandelt werden.

Evidenzgrad 1, Empfehlungsgrad B, starker Konsens

\section{EMPFEHLUNG 3.8}

Die rektale Anwendung von Mesalazineinläufen oder -schäumen $(\geq 1 \mathrm{~g} / \mathrm{d}$ ) soll der topischen Steroidtherapie vorgezogen werden.

Evidenzgrad 2, Empfehlungsgrad A, starker Konsens

\section{EMPFEHLUNG 3.9}

Bei oraler Gabe von Mesalazin freisetzenden Präparaten sollte die einmal tägliche Gabe vorgezogen werden.

Evidenzgrad 2, Empfehlungsgrad B, starker Konsens

\section{Hintergrund}

Die Erstlinientherapie einer leicht- bis mäßiggradigen Linksseitencolitis ulcerosa sollte aus einer Kombinationstherapie von oraler und rektaler Therapie mit 5-ASA bestehen, da sowohl höhere Ansprechraten als auch ein schnellerer Wirkungseintritt im Vergleich zur alleinigen oralen Therapie bestehen [250]. Sowohl die orale als auch die rektale 5-ASA-Gabe ist effektiver als Plazebo [235, 237, 250], sodass bei Intoleranz der rektalen Applikation eine alleinige orale Therapie erwogen werden kann; die Wirksamkeit wurde in verschiedenen Studien beschrieben. Die lokale Applikation erzielt höhere rektale Wirkstoffkonzentrationen [251]. Für die Gabe von Einläufen bzw. Schäumen sind keine signifikant divergenten Ansprechraten beschrieben [252]. Orales 5-ASA ist nicht effektiver als orales Sulfasalazin, ist aber mit weniger Nebenwirkungen behaftet [253]. Ein signifikanter Wirkungsunterschied der einzelnen 5-ASA-Präparationen scheint nicht zu bestehen [253, 254]. Die optimale tägliche Dosierung ist vermutlich von der Wahl des Präparats abhängig, auf eine ausreichend hohe Dosierung ( $\geq 3 \mathrm{~g} / \mathrm{d}$ ) sollte geachtet werden [253]. Die rektale Gabe von Beclomethasondipropionat scheint der lokalen Applikation von 5-ASA bei der Linksseitencolitis gleichwertig zu sein, was durch eine Metaanalyse bestätigt werden konnte [255]. Ebenso war die rektale Gabe von $2 \mathrm{~g}$ Budesonidschaum erfolgreich in der Remissionsinduktion [245]. Ein wichtiger Aspekt ist die Therapieadhärenz insbesondere der 5-ASA-Präparationen, da ein signifikanter Anteil fehlender Therapieeffekte auf eine mangelnde Einnahme zurückzuführen ist [256]. Unterschiedliche Dosierungsoptionen und Galenik (einmal tägliche Gabe; Tabletten bzw. Pellets) sollten bei der Verordnung berücksichtigt werden; die einmal hochdosierte tägliche Gabe ist der mehrfachen täglichen Einnahme nicht unterlegen [257, 258]. Die Bedeutung lokaler Therapien sollte mit dem Patienten erörtert werden.

\section{EMPFEHLUNG 3.10}

Eine systemische Steroidtherapie $(0,5-1 \mathrm{mg} / \mathrm{kg} \mathrm{KG} / \mathrm{d}$ Prednisolonäquivalent) soll begonnen werden, wenn die Symptome der CU nicht auf die unter 3.5.-3.9. genannte Therapie ansprechen.

Evidenzgrad 1, Empfehlungsgrad A, starker Konsens

Budesonid MMX $9 \mathrm{mg} / \mathrm{d}$ sollte bei leichter bis mäßig aktiver linksseitiger CU bei unzureichendem Ansprechen oder einer Unverträglichkeit von 5-Aminosalizylaten freisetzenden Präparaten eingesetzt werden.

Evidenzgrad 1, Empfehlungsgrad B, starker Konsens 


\section{Hintergrund}

Die Notwendigkeit einer oralen Steroidtherapie bei gering- bis mäßiggradig aktiver Colitis ulcerosa ist abhängig vom klinischen Ansprechen und der Toleranz einer 5-ASA-Therapie, ebenso von dem Patientenwunsch, wie auch von der Einschätzung des Arztes; eine klare Grenzlinie existiert nicht. In einer Studie war die mediane Zeit bis zum Sistieren der rektalen Blutungen 9 Tage bei $4,8 \mathrm{~g}$ 5-ASA/d, wobei eine anhaltende Remission erst nach 37-45 d auftrat [259]. Daher sollte eine Steroidtherapie bei Verschlechterung der klinischen Symptomatik unter Therapie oder bei Persistieren der Blutungen über mehr als $14 \mathrm{~d}$ erfolgen.

Orales Budesonid ohne MMX-Galenik ist in der Therapie der Colitis ulcerosa nicht wirksam [260], wohingegen Budesonid MMX sich in Studien als wirksam gezeigt hat. In der Core-I-Studie zeigte Budesonid MMX keinen Unterschied in der Effektivität zu 2,4 g Asacol [261]. In der Core-II-Studie waren $9 \mathrm{mg}$ Budesonid der Gabe von Plazebo signifikant überlegen [262]. In einer gepoolten Analyse der Core-I- und Core-II-Studie konnte gezeigt werden, dass v. a. Patienten mit einer milden und moderat aktiven Erkrankung von der Gabe von 9 mg Budesonid MMX profitieren, ebenso wie Patienten mit Linksseitencolitis [263]. Die zusätzliche Gabe von Budesonid MMX bei inadäquatem Ansprechen von 5-ASA war in einer weiteren Studie wirksam, sodass die zusätzliche Gabe in entsprechender Situation erfolgen kann; ein direkter Vergleich zu konventionellen Steroiden liegt nicht vor [264]. Das Sicherheitsprofil von Budesonid MMX hat sich in den Zulassungsstudien als günstig erwiesen mit Langzeitsicherheitsdaten, die mit den Plazebogruppen vergleichbar waren.

\section{Ausgedehnter Befall}

\section{EMPFEHLUNG 3.11}

Bei ausgedehntem Befall soll eine leichte bis mäßig schwere CU zunächst mit einem oralen Mesalazin freisetzenden Präparat in einer Dosierung $\geq 3 \mathrm{~g} / \mathrm{d}$ in Kombination mit Mesalazineinläufen oder -schäumen behandelt werden.

Evidenzgrad 1, Empfehlungsgrad A, starker Konsens

\section{EMPFEHLUNG 3.12}

Eine systemische Steroidtherapie $(0,5-1 \mathrm{mg} / \mathrm{kg} \mathrm{KG} / \mathrm{d}$ Prednisolonäquivalent) soll begonnen werden, wenn die Symptome der CU nicht auf die unter 3.5 - 3.9 und 3.11 genannten Therapien ansprechen oder bereits bei Diagnosestellung eine schwere Form der Colitis vorliegt.

Evidenzgrad 1, Empfehlungsgrad A, starker Konsens

\section{Hintergrund}

Die Therapieprinzipien bei ausgedehnter, gering- bis mäßiggradig aktiver Colitis ulcerosa entsprechen im Wesentlichen denen der Linksseitencolitis; die meisten Studien beinhalten beide Befallsmuster.
Die bessere Wirksamkeit der Kombination von oralem 5-ASA mit einer lokalen Therapie im Gegensatz zu einer oralen Monotherapie konnte in einer randomisierten Studie nachgewiesen werden [1]. Wie bei der Linksseitencolitis ist Sulfasalazin ebenso wirksam, jedoch mit mehr Nebenwirkungen assoziiert [253]. Die einmal tägliche Gabe ist unabhängig von der Formulierung genauso wirksam [254, 258]. Auf eine ausreichende Dosierung sollte geachtet werden ( $\geq 3 \mathrm{~g}$ ) [253].

Bei unzureichendem Ansprechen einer 5-ASA-Erhaltungstherapie sollte eine Steroidtherapie eingeleitet werden; eine eindeutige Indikationsstellung im Hinblick auf Ausprägung, Ausdehnung oder zeitliches Ansprechen besteht nicht. Unter bereits laufender Immunsuppression ist eine differenzierte Adaptation in Abhängigkeit von der Medikamentenhistorie notwendig. Die zusätzliche Gabe von $9 \mathrm{mg}$ Budesonid MMX war bei ausgedehntem Befall im Gegensatz zur Linksseitencolitis in zwei Studien der Plazebogabe nicht überlegen [263].

\section{Remissionserhaltung bei primär unkomplizierter Colitis ulcerosa}

\section{EMPFEHLUNG 3.13}

5-Aminosalizylate sollen primär als remissionserhaltende Therapie eingesetzt werden, wenn ein Ansprechen auf Aminosalizylate oder Steroide besteht.

Evidenzgrad 1, Empfehlungsgrad A, starker Konsens

\section{Hintergrund}

Die Evidenz für den Einsatz von 5-Aminosalizylaten zum Remissionserhalt bei Colitis ulcerosa ist gut. Gemäß einer aktuellen Metaanalyse mit Auswertung von 42 Studien und insgesamt 8928 Patienten ist orales 5-ASA Plazebo im Remissionserhalt nach klinischen und endoskopischen Kriterien signifikant überlegen [253]. Für die rektale Anwendung von 5-ASA erbrachte eine weitere Metaanalyse vergleichbare Ergebnisse, nach 12 Monaten war die Remissionsrate gegenüber Plazebo signifikant höher [265]. Insbesondere bei der distalen Colitis ulcerosa erweisen sich rektale 5-Aminosalizylate als effektiv, allerdings ist die Adhärenz bei rektaler Therapie sehr variabel [266].

Bisher gibt es keinen wissenschaftlichen Beleg für eine bessere Therapie als Aminosalizylate zum Remissionserhalt nach Ansprechen auf Steroide oder 5-Aminosalizylate [267]. Studien, die Aminosalizylate gegen Thiopurine oder Biologika im Remissionserhalt getestet haben, fehlen. Aufgrund des günstigeren Nebenwirkungsprofils empfehlen wir daher den primären Einsatz von Aminosalizylaten in oben genannter Situation.

Obwohl mittlerweile in drei kontrollierten Studien als effizient belegt, findet der primäre Einsatz von $E$. coli Nissle aufgrund der im Vergleich zu 5-ASA weniger umfangreichen Studienlage keine einheitliche Bewertung. In einer Metaanalyse über 4 randomisierte, kontrollierte und verblindete Studien zeigte sich kein Unterschied in der Remissionserhaltung zwischen Mesalazin und E. coli Nissle [268]. Aufgrund der deutlich weniger umfangreichen Studienlage im Vergleich zu 5-ASA werden weitere Studien gefordert, 
- Tab. 7 Aminosalizylat-Dosierungen mit nachgewiesener Wirkung im Remissionserhalt.

\begin{tabular}{|l|l|l|}
\hline Applikation & Dosis & Kommentar \\
\hline Orale Monotherapie & 5-ASA $\geq 2 \mathrm{~g} /$ Tag $[232]$ & $\begin{array}{l}\text { Kein klarer Dosis-Wirkungs-Effekt in mehreren Studien nachweisbar; } \\
\text { bevorzugt als Einmalgabe [258, 274, 276, 277, 279, 283] }\end{array}$ \\
\hline Rektale Monotherapie & 5-ASA Dosis $\geq 1 \mathrm{~g} /$ Tag $[280-282]$ & Keine Studien zum Dosis-Wirkungs-Effekt \\
\hline Oral-rektale Kombinationstherapie & $\begin{array}{l}\text { Intermitt. rektale 5-ASA-Gabe 1 -4 g } \\
\text { jeweils } 2 \times / \text { Woche; } \\
\text { orales 5-ASA täglich 1,5-3 g/Tag [284] }\end{array}$ & \\
\hline
\end{tabular}

um die Stellung von E. coli Nissle im Therapiealgorithmus besser zu definieren [269].

\section{EMPFEHLUNG 3.14}

Der Weg der Applikation von 5-Aminosalizylaten soll sich nach dem Befallsmuster der Erkrankung richten. Die Proktitis und die linksseitige Colitis sollten primär rektal therapiert werden.

Evidenzgrad 1, Empfehlungsgrad A, starker Konsens

\section{Hintergrund}

Die orale Anwendung wurde für alle Präparate untersucht, die rektale Anwendung nur in eingeschränkter Weise und in den verschiedenen Formulierungen (Suppositorien, Rektalschaum, Klysma) nur für 5-ASA. Bei der distalen CU ist die rektale Applikation Plazebo [236, 265] und insbesondere topischen Steroiden überlegen [242]. Die Kombination aus oraler und rektaler Therapie ist der oralen Monotherapie überlegen [270, 271]. In dieser Kombination kann die topische Therapie durchaus intermittierend angewandt werden [272]. Eine Alternative zur rektalen Applikation bei distaler Colitis und geringer Therapieadhärenz können 5-Aminosalizylat-Formulierungen der neueren Generation (bspw. Multimatrix-Formulierungen, Granula) mit gutem Freisetzungsprofil im linksseitigen Colon sein; sie sind den Aminosalizylaten älterer Generation mit ilealer Freisetzung in diesem Fall überlegen [247, $273,274]$.

\section{EMPFEHLUNG 3.15}

Eine Kombination von oralen und rektalen 5-Aminosalizylaten soll als Zweitlinien- Erhaltungstherapie verwendet werden. Evidenzgrad 1, Empfehlungsgrad A, starker Konsens

\section{Hintergrund}

In zwei kontrollierten Studien konnte eine Überlegenheit der Kombinationstherapie (orales und rektales 5-ASA) gegenüber einer alleinigen oralen 5-Aminosalizylat-Therapie hinsichtlich des Remissionserhalts dokumentiert werden [270, 271]. Kommt es unter einer oralen oder rektalen 5-ASA-Monotherapie zu einem Schub, der mittels oral-rektaler Kombinationstherapie erfolgreich behandelt wird, soll nachfolgend die Kombinationstherapie zur Remissionserhaltung fortgeführt werden. In beiden oben genannten Studien wurde die rektale Therapie jedoch lediglich zweimal wöchentlich mit einer Wochendosis von $8 \mathrm{~g}$ [270] bzw. $2 \mathrm{~g}$ [271] gegeben; somit unterscheiden sich die Dosierungen und Applikationsintervalle von den derzeit zur Remissionsinduktion empfohlenen Dosierungen.

\section{EMPFEHLUNG 3.16}

Zur Remissionserhaltung sollen Dosierungen der 5-Aminosalizylate verwendet werden, für die klinische Wirksamkeit nachgewiesen wurde ( $\triangleright$ Tab. 7).

Evidenzgrad 1, Empfehlungsgrad A, starker Konsens

Es soll bevorzugt eine tägliche Einmalgabe verabreicht werden. Evidenzgrad 2, Empfehlungsgrad A, starker Konsens Angesichts des Nebenwirkungsprofils bei vergleichbarer Effizienz sollte Mesalazin der Vorzug gegenüber Sulfasalazin gegeben werden.

Evidenzgrad 1, Empfehlungsgrad B, starker Konsens

\section{Hintergrund}

Für den Remissionserhalt mit 5-Aminosalizylaten konnte bisher keine klare Dosis-Wirkungs-Beziehung herausgearbeitet werden. In einer aktuellen Metaanalyse war die tägliche Einnahme von 1,2 g 5-ASA statistisch nicht schlechter im Remissionserhalt nach 12 Monaten als eine Tagesdosis von 2,4 g [253]. Allerdings fiel auf, dass Patienten mit der höheren Tagesdosis von $2,4 \mathrm{~g}$ deutlich länger in Remission blieben (median 175 Tage) als Patienten mit einer Tagesdosis von 1,2 g (median 129 Tage); noch deutlicher wurde dies in der Subgruppenanalyse von Patienten mit ausgedehntem Befall (2,4 g/Tag: mediane Remission 143 Tage vs. 47 Tage bei 1,2 g). Dieser Unterschied war nach 12 Monaten jedoch nicht mehr signifikant. In einer Metaanalyse aus 2011 erwies sich eine Tagesdosis 5-ASA $\geq 2 \mathrm{~g}$ einer Tagesdosis von $<2 \mathrm{~g}$ im Remissionserhalt überlegen, allerdings wurden dazu Studien mit unterschiedlichen 5-ASA Präparaten ausgewertet (Mesalazin, Olsalazin, Sulfasalazin und Balsalazid) [232]. Auch in einer Cochrane-Analyse aus 2016 zeichnete sich für eine 5-ASA-Dosis $\geq 2 \mathrm{~g} /$ Tag ein Trend zum besseren Remissionserhalt ab [253]. Zusammengefasst sollte die 5-ASA-Dosis zum Remissionserhalt $\geq 2 \mathrm{~g} /$ Tag betragen. Unklar ist jedoch weiterhin, ob Patienten, die zur Remissionsinduktion 
eine höhere Dosis benötigten bzw. häufigere Schübe in der Vergangenheit hatten, auch in der Remissionserhaltung höhere 5-ASA-Dosen benötigen. Hingegen scheinen höhere 5-ASA-Do sierungen nicht mit vermehrten Nebenwirkungen einherzugehen [275].

Eine einmal tägliche Einnahme von 5-ASA-Präparaten erwies sich in mehreren Studien der aufgeteilten Dosis als ebenbürtig hinsichtlich des Remissionserhalts [258, 274, 276 - 279] und sollte daher im Hinblick auf die Adhärenz bevorzugt werden. Bezüglich der rektalen Applikation von 5-ASA existieren keine Daten, die eine Dosis-Wirkungs-Beziehung nahelegen. Die meisten Studien, in denen 5-ASA rektal (Suppositorien, Schäume) als Monotherapie bei Erwachsenen verabreicht wurde, setzten Dosierungen von $1 \mathrm{~g} /$ Tag ein [280 - 282] und konnten eine Überlegenheit gegenüber Plazebo zeigen. Im Fall einer oral-rektalen 5-ASA-Kombinationstherapie wurde eine Wirksamkeit von rektalem 5-ASA für die zweimal wöchentliche Gabe von jeweils $4 \mathrm{~g}$ [270] bzw. jeweils $1 \mathrm{~g}$ [271] gezeigt.

Sulfasalazin erwies sich in einer Cochrane-Analyse im Vergleich zu anderen 5-ASA-Präparaten im Remissionserhalt als überlegen [253], wobei der Unterschied gering war (Odds Ratio 1,1; 95\%-KI $1,03-1,27)$. Zudem lag kein signifikanter Unterschied im Nebenwirkungsprofil zwischen Sulfasalazin und den anderen 5-ASA-Präparaten vor, was im Kontrast zur klinischen Routine steht. Da in die meisten Studien Patienten mit bestehender SulfasalazinTherapie eingeschlossen wurden, ist jedoch nicht auszuschließen, dass ein Selektionsbias vorlag.

\section{EMPFEHLUNG 3.17}

Eine remissionserhaltende Therapie mit 5-Aminosalizylaten sollte bei Effektivität mindestens 2 Jahre durchgeführt werden. Evidenzgrad 2, Empfehlungsgrad B, starker Konsens

\section{Hintergrund}

Zur Frage, ob und wann eine remissionserhaltende Dauertherapie einer nur beobachtenden Haltung überlegen ist, gibt es nur einzelne wissenschaftliche Daten. Eine kontrollierte Studie an 112 Patienten zeigte, dass bei Patienten mit einer Remissionsdauer von ein bis zwei Jahren eine Fortführung der 5-Aminosalizylat-Therapie über 12 Monate das Auftreten von Rezidiven im Vergleich zu Plazebo vermindern kann [285]. Bestand die Remission schon länger als 2 Jahre, profitierten die Patienten nicht von einer weiteren 12-monatigen 5-ASA-Therapie. Aus methodischen und statistischen Gründen (notwendige Gruppengrößen für volle statistische Aussagekraft) kann man die Ergebnisse dieser Untersuchung jedoch nicht als definitive Aussage zum Stellenwert einer remissionserhaltenden 5-ASA-Therapie bei Patienten mit über 2-jähriger Remission werten; zudem wurde mit 1,2 g 5-ASA eine Tagesdosis verwendet, die nicht der aktuellen Empfehlung entspricht.

In seltenen Fällen kann es unter 5-ASA zu Nephrotoxizität kommen. Eine Nachfrage bei 118 englischen und 45 internationalen CED-Zentren erbrachte 151 gesicherte Fälle mit einer Nierenschädigung, die im Mittel nach 3 Jahren Therapie auftrat. Es wurde eine genetische Disposition identifiziert [286]. Eine evidenzbasierte Empfehlung zu Zeitpunkt bzw. nachfolgenden Intervallen der Überprüfung der Nierenwerte unter Langzeittherapie existiert nicht; diese kann alle 6 bis 12 Monate erfolgen [287].

Besonders zu beachten ist die schlechte Therapieadhärenz mit etwa $50 \%$ bei einer Langzeittherapie [288].

\section{EMPFEHLUNG 3.18}

Eine Langzeittherapie mit 5-ASA sollte den CU-Patienten unter dem Aspekt der Karzinomprävention angeboten werden. Evidenzgrad 2, Empfehlungsgrad B, Konsens

\section{Hintergrund}

In mehreren Kohorten- sowie Fall-Kontroll-Studien konnte gezeigt werden, dass sowohl Sulfasalazin als auch 5-ASA mit einem verminderten kolorektalen Karzinomrisiko bei Patienten mit einer Colitis ulcerosa verbunden sind [181, 289]. Eine Analyse der Risikofaktoren für ein KRK bei CU von Velayos [181] zeigte eine Effektivität für 5-ASA in der Chemoprävention im Hinblick auf die Entwicklung von kolorektalen Karzinomen, wobei diese Reduktion auch statistisch signifikant war. Der Effekt von 5-ASA zur Chemoprävention bei Colitis ulcerosa ist nicht nur begrenzt auf HighRisk-Patienten [158, 192, 290], sodass die Empfehlung zu einer langjährigen Chemoprävention für alle Patienten außer jenen mit einer isolierten Proktitis gilt [101, 158, 178, 190, 192, 290 - 298].

Immunsuppressiva wie Azathioprin können theoretisch durch die Unterdrückung der Schleimhautentzündung auch karzinompräventiv wirken. Das Gleiche gilt auch für MTX und Anti-TNF. Die Datenlage dazu ist aber nicht eindeutig [178, 181, 190, 229, 291, 299 - 302], auch wenn eine Observationskohortenstudie der CESAME-Gruppe zeigte, dass Patienten mit einer langen Azathioprin-Therapie eher ein geringeres Risiko für ein kolorektales Karzinom hatten [303]. Zusammenfassend ergibt sich keine ausreichend sichere Evidenz zur Empfehlung der Thiopurine zur Chemoprävention bei Colitis-ulcerosa-Patienten.

\section{EMPFEHLUNG 3.19}

Bei erneuten Schüben soll die remissionserhaltende Therapie eskaliert werden.

Evidenzgrad 2, Empfehlungsgrad A, starker Konsens

\section{EMPFEHLUNG 3.20}

Möglichkeiten zur stufenweisen remissionserhaltenden Therapieeskalation sind eine Dosiseskalation einer oralen/rektalen Kombinationstherapie mit Aminosalizylaten (Evidenzgrad 1, Empfehlungsgrad A), eine Anti-TNF-Therapie (Evidenzgrad 1, Empfehlungsgrad A), eine Therapie mit Vedolizumab (Evidenzgrad 1, Empfehlungsgrad A) oder eine Therapie mit Thiopurinen (Evidenzgrad 2, Empfehlungsgrad B).

Starker Konsens 


\section{Hintergrund}

Kommt es unter einer remissionserhaltenden Therapie zu einem erneuten Schub, sollen die Möglichkeiten zur Therapieeskalation überprüft werden. Obwohl die Zahl verfügbarer medikamentöser Therapien bei Colitis ulcerosa gestiegen ist und die Möglichkeit der Kombinationstherapie für gewisse Präparate vorliegt, ist die Zahl der effektiven Therapien weiterhin limitiert, sodass die verfügbaren Therapien in einer optimalen Dosierung und in optimalen Dosierungsintervallen angewendet werden sollten. Neben einer Dosiseskalation einer bestehenden Therapie mit Aminosalizylaten stellen Therapien mit Thiopurinen, TNF-Antikörpern und Vedolizumab inkl. Dosis-Intervall-Anpassungen weitere Möglichkeiten der Eskalation dar. Bei der Wahl der jeweiligen Eskalationsstrategie sollten die bisherigen Therapien, eventuelle Unverträglichkeiten und Komorbiditäten berücksichtigt werden. Es gibt jedoch weder Vergleichsstudien zwischen einer Immunsuppression und einer definierten (optimierten) Basistherapie, noch zwischen den einzelnen Substanzgruppen, und es fehlen formale Eskalationsstudien. Mangels direkter Vergleichsstudien ist die wirksamste Therapiestrategie nicht klar. Bezüglich ausführlicher Informationen zum Einsatz von Thiopurinen, TNF-Antikörpern und Vedolizumab sei auf das Kapitel Remissionserhalt bei kompliziertem Verlauf der Colitis ulcerosa (Empfehlungen 3.33 - 3.34) verwiesen.

\section{EMPFEHLUNG 3.21}

Kortikosteroide sollen zur Remissionserhaltung nicht eingesetzt werden.

Evidenzgrad 1, Empfehlungsgrad A, starker Konsens

\section{Hintergrund}

Für die Wirksamkeit einer Remissionserhaltungstherapie durch Kortikosteroide gibt es sowohl in der lokalen Anwendung [304] als auch in der systemischen Applikation keine Evidenz [305]. Aufgrund des hormonellen Charakters der Steroide treten in der Langzeittherapie regelmäßig schwere unerwünschte Wirkungen wie Osteoporose und Katarakt auf. Daneben besteht unter Kortikosteroiden in der Monotherapie, besonders aber in der Kombinationstherapie das Risiko schwerer infektiöser Komplikationen [306].

\section{Komplizierte Verlaufsformen der Colitis ulcerosa/ Schwere Colitis ulcerosa}

\section{EMPFEHLUNG 3.22}

Patienten mit einem schweren akuten Schub einer Colitis ulcerosa sollten stationär behandelt werden.

Evidenzgrad 1, Empfehlungsgrad B, starker Konsens

Die Behandlung sollte in enger Zusammenarbeit in einem interdisziplinären Team inkl. eines erfahrenen Abdominalchirurgen erfolgen.

Evidenzgrad 3, Empfehlungsgrad B, starker Konsens

\section{Hintergrund}

Eine mehr oder weniger einfach anwendbare und im klinischen Alltag bewährte Definition der schweren Colitis ulcerosa kann anhand der Kriterien von Truelove und Witts erfolgen [83, 307]. Diese Kriterien beinhalten:

- schwere Diarrhöen mit 6 oder mehr makroskopisch blutigen Stühlen proTag,

- Fieber (mit einer mittleren Abendtemperatur von über $37,5^{\circ}$ Celsius oder einer Temperatur $>37,8^{\circ}$ Celsius an wenigstens 2 von 4 Tagen,

- Tachykardie mit einem Puls $>90 /$ min,

Anämie mit einem Hb-Wert $<75 \%$ der Norm und

- eine BSG > $30 \mathrm{~mm} / \mathrm{h}$.

Diese Parameter bilden im Prinzip eine systemische Krankheitsaktivität ab, die sich in der Regel auch direkt aus dem klinischen Bild ergibt. Es handelt sich dabei um schwer kranke Patienten. Die Anwendung von Score-Systemen spielt bei ihnen im klinischen Alltag eher eine bestätigende Rolle und ist nicht zwingend geboten. Allerdings ist grundsätzlich auf Zeichen einer systemischen Krankheitsmanifestation zu achten. Bei Kindern sollte der PUCAI zur Anwendung kommen [85]. In den PUCAI fließen ein: Bauchschmerzen, rektaler Blutabgang, Stuhlkonsistenz, Stuhlfrequenz, nächtlicher Stuhlgang und die Aktivitätsbeeinträchtigung (Summenbildung mit einem Wert zwischen 0 und 85). Ein PUCAI von > 65 Punkten zeigt einen schweren Colitisschub an.

Entscheidend ist, bei den Patienten frühzeitig die richtige Diagnose zu stellen und intestinale Infektionen auszuschließen [308]. Die schwere Colitis ulcerosa stellt nach wie vor ein bedrohliches Krankheitsbild dar, obwohl die Mortalitätsrate in spezialisierten Zentren $<1 \%$ liegt [84]. Daher ist die stationäre Behandlung dieser Patienten erforderlich. Eine Metaanalyse, die das Ansprechen der schweren Colitis ulcerosa auf Kortikosteroide untersuchte, zeigte eine mittlere Proktokolektomierate von $27 \%$ und eine Mortalitätsrate von $1 \%$ [309]. Dies trifft insbesondere für Patienten mit einem Lebensalter > 60 Jahre zu, da gezeigt werden konnte, dass die Mortalität in dieser Situation erhöht ist [310].

\section{Konventionelle Therapie}

\section{EMPFEHLUNG 3.23}

Ein schwer verlaufender Schub einer Colitis ulcerosa soll mit einer systemischen Steroidtherapie (z. B. 1 mg/kg KG Prednisolonäquivalent pro Tag) behandelt werden. Evidenzgrad 2, Empfehlungsgrad A, starker Konsens

\section{EMPFEHLUNG 3.24}

Patienten mit einem schweren akuten Schub sollten eine Thromboseprophylaxe erhalten.

Evidenzgrad 3, Empfehlungsgrad B, starker Konsens 


\section{EMPFEHLUNG 3.25}

Zur Beurteilung des Ansprechens der systemischen Steroidtherapie sollten das klinische Bild und objektivierbare Parameter herangezogen werden.

Evidenzgrad 4, Empfehlungsgrad B, starker Konsens

\section{Hintergrund}

Steroide stellen in dieser Situation seit Mitte des letzten Jahrhunderts die Standardtherapie dar [83, 311 - 315]. Basierend auf mehreren Studien wird eine Dosis von $1 \mathrm{mg}$ Prednisolon/kg KG/ Tag empfohlen; höhere Dosierungen bringen keinen zusätzlichen Nutzen [316]. Eine Therapiedauer unter drei Wochen ist mit einer erhöhten Rückfallquote assoziiert; weiter gelten Dosierungen unter $15 \mathrm{mg}$ Prednisolon/Tag als ineffektiv [311]. Grundsätzlich kann sowohl eine orale als auch eine intravenöse Gabe erfolgen; bei erheblicher Motilitätsstörung sollte die intravenöse Gabe bevorzugt werden. Zu den objektiven Parametern, die bezüglich des Ansprechens auf eine Therapie mit Steroiden herangezogen werden sollten, gehören u. a. die Stuhlfrequenz, Blutbeimengungen im Stuhl, Hb-Wert, Ultraschallbefund, Endoskopiebefund, CRP, Blutbild und fäkale Neutrophilenmarker.

Bei Kontraindikationen zur Steroidtherapie oder Intoleranz kommen Infliximab, Ciclosporin A oder Tacrolimus infrage. Da die Mehrheit der Studien zu diesen Substanzen in der steroidrefraktären Situation durchgeführt wurde, soll auf die Daten in diesem Abschnitt eingegangen werden.

Es ist entscheidend, dass bei einem Nichtansprechen frühzeitig (nach etwa drei Tagen) eine Therapieeskalation bzw. ein Wechsel der Therapiestrategie erwogen wird. Dies ist von Bedeutung, da gezeigt werden konnte, dass Patienten, die mit einer nicht effektiven Medikation inklusive Steroiden behandelt wurden, nicht nur eine erhöhte Morbidität aufwiesen, sondern auch verzögert operiert wurden [317 - 320].

Die notwendige Therapie geht über die pharmakologische Therapie weit hinaus und kann nur unter stationären Bedingungen gewährleistet werden, da neben der medikamentösen Therapie bei diesen Patienten vorab generelle und spezifische Maßnahmen erforderlich sein können. Dazu gehören engmaschige Laborwertkontrollen, mikrobiologische Untersuchungen (Reiseanamnese erheben), physio- und ggf. psychotherapeutische Betreuung und Bluttransfusionen [321]. Darüber hinaus gehören dazu:

1. parenteraler Flüssigkeits- und Elektrolytausgleich. Insbesondere eine Hypokaliämie sowie eine Hypomagnesiämie sollten vermieden werden, da beide Zustände eine intestinale Hypomotilität und damit ein toxisches Megakolon begünstigen [322].

2. Alle motilitätshemmenden sowie sonstige potenziell Mukosa schädigende Medikamente sollten abgesetzt werden, da sie ebenfalls ein toxisches Megakolon begünstigen [6, 323 - 325].

3. eine Sigmoidoskopie, um die Diagnose zu bestätigen und gleichzeitig Biopsien abzunehmen, die eine intestinale CMVReaktivierung ausschließen lassen (zusammen mit der systemischen CMV-Viruslast). Dies spielt insbesondere in der steroidrefraktären Situation eine Rolle; die CMV-Replikation sollte in dieser Situation behandelt werden [326-331].
4. Ausschluss einer Infektion mit Clostridium difficile, die gehäuft bei Patienten mit einer schweren Colitis ulcerosa auftritt und mit einer erhöhten Mortalität und Morbidität assoziiert ist [52, 310, 332 - 337]. Wenn ein positiver Clostridium-difficileNachweis erfolgt, sollte entsprechend der DGVS-Leitlinie behandelt werden (s. Kapitel 4) [338]. Ob die immunsuppressive Therapie in dieser Situation gestoppt werden soll, ist nicht klar, die Daten dazu sind kontrovers [339, 340].

5. eine Thromboseprophylaxe mit einem niedermolekularen Heparin, da das Thromboserisiko bei einem akuten Schub unabhängig von anderen Risikofaktoren deutlich erhöht ist [337, 341 - 344].

6. eine Ernährungstherapie bei Patienten mit einer Malnutrition. Diese sollte vorzugsweise enteral durchgeführt werden, da diese mit weniger Komplikationen (9 versus $35 \%$ ) assoziiert ist [345] und die parenterale nicht mit einem besseren Endergebnis assoziiert ist [346].

Bei Kindern liegt das Risiko, wegen eines schweren Colitisschubs stationär aufgenommen zu werden, bei $30-40 \%$ bis zum 16. Lebensjahr und damit höher als bei Erwachsenen. Bei Kindern und Jugendlichen sollte Methylprednisolon einmal täglich in einer Dosis von $1-2 \mathrm{mg} / \mathrm{kg} / \mathrm{d}$ i. v., maximal $40-60 \mathrm{mg} / \mathrm{d}$ gegeben werden [309]. Etwa 30 - $40 \%$ der pädiatrischen Patienten mit schwerer Colitis ulcerosa sprechen nicht auf die initiale Therapie an und benötigen eine Therapieeskalation mit Infliximab oder Cyclosporin. Bei einem PUCAI über 45 Punkten am Tag 3 der i. v. Steroidtherapie sollten eine weitere Diagnostik (Sigmoidoskopie mit Ausschluss einer CMV-Colitis) veranlasst und Vorbereitungen für eine erweiterte Therapie getroffen werden. Beträgt der PUCAI $>65$ Punkte am Tag 5, empfiehlt es sich, die Therapie zu erweitern, da ein Ansprechen wenig wahrscheinlich ist. Alternativ ist stets eine Kolektomie mit Eltern und Patient zu diskutieren. Für Kinder und Jugendliche wurde von der ECCO (European Crohn's and Colitis Organization) und der ESPGHAN (European Society of Pediatric Gastroenterology, Hepatology, and Nutrition) eine separate Leitlinie zur Behandlung des schweren Schubs herausgegeben [347].

\section{Colitis ulcerosa refraktär auf systemische Steroidtherapie}

\section{EMPFEHLUNG 3.26}

Patienten mit einer Colitis ulcerosa mit mittelschwerer bis schwerer Krankheitsaktivität, die unzureichend auf die Behandlung mit systemischen Steroiden ansprechen bzw. bei denen Kontraindikationen oder Intoleranzen vorliegen, sollten mit TNF-Antikörpern (Evidenzgrad 2, Empfehlungsgrad B) oder mit Ciclosporin A (Evidenzgrad 1, Empfehlungsgrad B) oder Tacrolimus (Evidenzgrad 2, Empfehlungsgrad B) behandelt werden. Im Fall von Infliximab sollte vorzugsweise eine Kombinationstherapie mit einem Thiopurin eingesetzt werden (Evidenzgrad 2, Empfehlungsgrad B). Bei der Therapieentscheidung sollte eine Proktokolektomie mit in Erwägung gezogen werden (Evidenzgrad 5, Empfehlungsgrad B).

Starker Konsens 


\section{EMPFEHLUNG 3.27}

Patienten mit einer Colitis ulcerosa mit fulminanter Krankheitsaktivität, die refraktär auf die Behandlung mit intravenösen Steroiden sind, sollten mit Infliximab (vorzugsweise als Kombinationstherapie mit einem Thiopurin) (Evidenzgrad 2, Empfehlungsgrad B) oder mit Ciclosporin A (Evidenzgrad 1, Empfehlungsgrad B) oder Tacrolimus (Evidenzgrad 2, Empfehlungsgrad B) behandelt werden. Eine Proktokolektomie sollte mit in Erwägung gezogen werden (Evidenzgrad 5, Empfehlungsgrad B).

Starker Konsens

\section{EMPFEHLUNG 3.28}

Tritt unter oben (3.27) genannter Therapie eine klinische Zustandsverschlechterung ein, sollte eine Proktokolektomie durchgeführt werden (Evidenzgrad 4, Empfehlungsgrad B). Die Proktokolektomie kann ebenso indiziert sein, wenn nach 4 - 7 Tagen keine Verbesserung des klinischen Zustands eintritt.

Starker Konsens

\section{Hintergrund}

Bei einer Colitis ulcerosa mit steroidrefraktärem Verlauf sollten infektiöse Ursachen wie $C$. difficile oder Cytomegalievirus ausgeschlossen werden (s. Kapitel 4). Eine intravenöse Steroidtherapie hat sich u. a. in einer retrospektiven Studie mit 110 Patienten als effektiver bei oral steroidrefraktärer, gesicherter Colitis ulcerosa gezeigt [348, 349]. Allerdings zeigte fast die Hälfte der Patienten in dieser Studie später einen steroidabhängigen Krankheitsverlauf. Für die TNF-Antikörper wurde ein klarer Nutzen bei jenen Patienten mit steroidabhängigem Krankheitsverlauf mit dem Erreichen einer steroidfreien Remission gezeigt, die zum Studienbeginn Steroide einnahmen (s. Kommentare zu Empfehlung 3.29) [350 - 353]. In die genannten Studien wurden auch Patienten mit steroidrefraktärem Krankheitsverlauf eingeschlossen, und es konnte eine Wirksamkeit an verschiedenen Endpunkten belegt werden. Obwohl der Anteil von Patienten mit Steroidtherapie und Grenzwerten für die Steroiddosis bei Studienbeginn jeweils erfasst wurde, sind möglicherweise die Steroiddosen vor Studienbeginn suboptimal gewesen, und es ist nicht immer möglich, steroidrefraktäre von steroidabhängigen Patienten zu differenzieren und die Studiendaten getrennt für diese Gruppen zu analysieren. Verschiedene für die CU wirksame TNF-Antikörper können daher auch in der steroidrefraktären Situation eingesetzt werden. Bei der Wahl der TNF-Antikörper bei steroidrefraktären Verlaufsformen ist in der klinischen Situation letztlich die Aktivität der Erkrankung von entscheidender Bedeutung. Während bei den weniger aktiven Erkrankungsformen mit geringerem Remissionsdruck unterschiedliche TNF-Antikörper, einschließlich Infliximab, Adalimumab oder Golimumab, eingesetzt werden können, sind für die fulminante Manifestation nur Infliximab oder die Calcineurininhibitoren kontrolliert untersucht worden. Für die Differenzie- rung der Aktivität der Erkrankung gibt es keine einheitlichen Kriterien, sodass diese letztlich der individuellen klinischen Einschätzung unterliegt. Die letzten Jahre haben zahlreiche kontrollierte Studien für die fulminante steroidrefraktäre Situation geliefert, auf die weiter unten im Detail eingegangen werden soll. Die Schwierigkeit bleibt jedoch, ein Ansprechen bzw. ein NichtAnsprechen auf eine Therapie zu beurteilen und rechtzeitig auch die Entscheidung für oder gegen ein operatives Vorgehen zu treffen. Im Folgenden soll die Bedeutung der Faktoren diskutiert werden, die uns bei der klinischen Beurteilung helfen sollen. Bei der klinischen Evaluation zeigte sich, dass bei einer Stuhlfrequenz von > 12/Tag nach 2 Tagen Behandlung mit intravenösen Steroiden eine Proktokolektomierate von 55 \% beobachtet wurde [354]. Die Oxford-Kriterien beurteilen den Tag drei; besteht dann eine Stuhlfrequenz von $>8 /$ Tag oder $3-8 /$ Tag zusammen mit einem CRP $>45 \mathrm{mg} / \mathrm{l}$, erfolgt eine Proktokolektomie in 85 \% während dieses stationären Aufenthalts [355]. Bei den Laborparametern konnte in einer prospektiven Arbeit mit 67 Patienten gezeigt werden, dass eine BSG $>75 \mathrm{~mm} / \mathrm{h}$ oder eine Körpertemperatur $>38^{\circ} \mathrm{C}$ bei Aufnahme mit einem 5- bis 9-fach erhöhten Risiko für eine Proktokolektomie assoziiert ist [356]. In der Radiologie sollte man sich auf die Daten beschränken, die in der Praxis heute noch relevant sind. Dazu zählt die Kolondilatation $>5,5 \mathrm{~cm}$, gemessen bei einer Abdomenübersichtsaufnahme, die mit einer Proktokolektomierate von $75 \%$ assoziiert wurde [354]. Damit übereinstimmend zeigt eine retrospektive Studie, dass der Nachweis eines Ileus mit einer Proktokolektomierate von $74 \%$ assoziiert war [357]. Die Daten für die endoskopische Beurteilung als prädiktiver Marker für den weiteren Verlauf beschränken sich auf kleine Fallserien [71, 358]. Die ausgeprägtesten Läsionen sind bei der Colitis ulcerosa im distalen Kolon lokalisiert, womit eine Sigmoidoskopie zur Beurteilung ausreichend ist [359]. Im klinischen Alltag ist es entscheidend, das Gesamtbild zu beurteilen. Retrospektive Daten zeigen, dass tiefe Ulzerationen, Steroidrefraktärität, Kolondilatation sowie Hypalbuminämie $(<30 \mathrm{~g} / \mathrm{dl})$ in hohem Maße $(85 \%)$ mit der Notwendigkeit einer Proktokolektomie assoziiert sind [360].

Nachfolgend werden die zur Verfügung stehenden medikamentösen Optionen erläutert.

Antibiotika Es liegen zwei Arbeiten vor, die die Frage adressieren, ob eine begleitende Antibiotikatherapie erfolgen sollte. In einer Arbeit wurde die Antibiotikatherapie bei 30 Patienten mit einer Colitis ulcerosa in einem Open-Label-Design untersucht. Die Gabe von Amoxicillin, Tetracyclin und Metronidazol über zwei Wochen schien hier einen Effekt bei der steroidrefraktären Colitis ulcerosa zu haben [361]. Im Gegensatz dazu zeigte eine frühere randomisierte plazebokontrollierte Studie an 39 Patienten mit schwerer Colitis ulcerosa keinen Effekt mit Metronidazol und Tobramycin [362].

Ciclosporin Es liegen mittlerweile mehrere Arbeiten vor, die die Wirksamkeit von Ciclosporin in dieser Situation belegen. Historisch war in einer ersten randomisierten und plazebokontrollierten Studie, die noch $4 \mathrm{mg} / \mathrm{kg} \mathrm{KG} / \mathrm{Tag}$ intravenös einsetzte, eine schnelle Wirksamkeit von Ciclosporin A in der Behandlung der steroidrefraktären Colitis ulcerosa beschrieben worden [363]. Limitierend ist, dass nur 20 Patienten in die Studie eingeschlossen wurden, wobei 9 von 11 Patienten unter Ciclosporin ansprachen und keiner der 9 Patienten in der Plazebogruppe ansprach [363]. 
Es folgte die randomisierte, doppelt verblindete Studie von d'Haens und Kollegen, die die Frage beantworten sollte, ob Ciclosporin A eine Alternative zur Kortikosteroidtherapie darstellen kann. Es wurden 30 Patienten mit schwerer Colitis ulcerosa entweder mit $40 \mathrm{mg}$ Methylprednisolon/Tag oder mit $4 \mathrm{mg} / \mathrm{kg} \mathrm{KG/}$ Tag Ciclosporin A intravenös behandelt. Nach 8 Tagen konnte bei 8/15 Patienten der Methylprednisolongruppe und bei 9/14 Patienten der Ciclosporin-A-Gruppe ein Therapieansprechen verzeichnet werden [364]. Es folgte die Arbeit, die $4 \mathrm{mg} / \mathrm{kg} \mathrm{KG/Tag}$ mit $2 \mathrm{mg} / \mathrm{kg} \mathrm{KG/Tag} \mathrm{Ciclosporin} \mathrm{verglich} \mathrm{und} \mathrm{keinen} \mathrm{Unterschied}$ in Bezug auf das Ansprechen an Tag 8 zeigen konnte [365]. Obwohl nicht alle Patienten beim Studieneinschluss einen steroidrefraktären Verlauf aufwiesen, hat sich die Dosierung 2 mg/kg KG Ciclosporin heute als Standard durchgesetzt. In der Studie wurden die Serumspiegel gemessen und die Ciclosporin-Konzentrationen ggf. angepasst. Die optimalen therapeutischen Serumspiegel sind nicht bekannt, anzustreben sind Spiegel zwischen 250 und $400 \mathrm{ng} / \mathrm{ml}$. Die Dosisanpassung erfolgt über Messungen der Talspiegel. Die beiden aktuellsten und größten Arbeiten unterstreichen diese Ergebnisse. Die Studie der französischen Studiengruppe randomisierte 115 Patienten mit steroidrefraktärer Colitis ulcerosa entweder in den Ciclosporin-Arm ( $2 \mathrm{mg} / \mathrm{kg} \mathrm{KG}$ ) oder in den Infliximab-Arm [366]. Ziel war es zu zeigen, dass Infliximab Ciclosporin nicht unterlegen ist, primärer Endpunkt war daher Therapieversagen an Tag sieben. Die Daten zeigen, dass Ciclosporin nicht wirksamer ist als Infliximab. Die CONSTRUCT-Studie hat bei 135 steroidrefraktären Patienten Ciclosporin ( 2 mg/kg KG) mit Infliximab verglichen und konnte ebenfalls für Infliximab keine Unterlegenheit nachweisen [367]. Die Proktokolektomierate in dieser Studie betrug $25 \%$ während des stationären Aufenthalts, $30 \%$ innerhalb von drei Monaten und $45 \%$ innerhalb des ersten Jahres [367]. Diese aktuellen Daten sind in der Metaanalyse von 2005 nicht enthalten, womit deren Schlussfolgerung, dass Ciclosporin der Standardtherapie, also Steroiden, nicht überlegen ist, kaum zu halten ist [368]. Limitierend sind die mit Ciclosporin assoziierten Nebenwirkungen, die den Einsatz im klinischen Alltag einschränken.

Fasst man die Daten der verschiedenen kontrollierten und unkontrollierten Studien zusammen, so kann mit dem Einsatz von Ciclosporin zumindest kurzfristig die Proktokolektomie in $76-85 \%$ der Patienten verhindert werden [363 - 365, 369, 370]. In zwei Arbeiten mit 76 und 142 Patienten, die Ciclosporin erhalten hatten, kam es über sieben Jahre in $58 \%$ und 88 \% zur Proktokolektomie [371, 372]. Mehrere Arbeiten zeigen, dass der Übergang von Ciclosporin zu einer Thiopurin-Therapie signifikant das Risiko für eine spätere Proktokolektomie senkt [370 - 373].

Tacrolimus Die Daten zu Tacrolimus beschränken sich auf wenige Arbeiten. Es liegt eine randomisierte plazebokontrollierte Studie vor, die zwei Serumkonzentrationen in der steroidrefraktären Situation verglichen hat $(5-10 \mathrm{ng} / \mathrm{ml}$ und $10-15 \mathrm{ng} / \mathrm{ml})$. Es konnte eine dosisabhängige Wirksamkeit nachgewiesen werden, jedoch war die Studie nicht aussagekräftig, um signifikante Unterschiede bei schwerer Colitis ulcerosa zu detektieren. Die Nebenwirkungen traten insbesondere in der Hochdosisgruppe auf [374]. Die zweite Studie ist ebenfalls eine randomisierte plazebokontrollierte Studie über zwei Wochen, die zeigen konnte, dass orales Tacrolimus signifikant Plazebo bei steroidrefraktären Colitis-ulce-
rosa-Patienten überlegen ist [375]. Zu diesem Ergebnis kommt auch eine Metaanalyse, die beide Studien einschließt [376]. Wie die Langzeitprognose in Bezug auf die Operation ist, ist aufgrund der eingeschränkten Patientenzahlen nur schwierig zu beurteilen. Die proktokolektomiefreien Raten nach 1, 3, 6 und 12 Monaten lagen bei $86 \%, 84 \%, 78 \%$ und 69\% [376]. Nach 44 Monaten waren $57 \%$ der Patienten nicht proktokolektomiert. Verschiedene retrospektive Studien liegen vor [377]. In einer Metaanalyse zeigten sich signifikant höhere klinische Ansprechraten unter Tacrolimus als unter Plazebo ( $R R=4,61,95 \%-K I ~ 2.09-10.17$, $\mathrm{p}=0.15 \times 10(-3))$. Der Anteil der Patienten ohne zwischenzeitlich erfolgte Proktokolektomie nach 1, 3, 6 und 12 Monaten war $86 \%$, $84 \%, 78 \%$ und $69 \%$ [376]. In einer offenen Studie an 100 Patienten mit mittelschwerer bis schwerer CU wurde die Therapie mit Tacrolimus mit einer Anti-TNF-Therapie verglichen; es zeigten sich vergleichbare Wirksamkeits- und Sicherheitsdaten [378]. Aus diesen Daten und der klinischen Praxis kann geschlossen werden, dass auch Tacrolimus als Therapiealternative zur konventionellen Steroidtherapie infrage kommt.

Infliximab In zwei randomisierten, doppelt verblindeten, plazebokontrollierten Studien (ACT 1 und 2) konnte zunächst generell die Wirksamkeit von Infliximab in der Behandlung der Colitis ulcerosa nachgewiesen werden [350]. In der ACT-1-Studie wurden 364 Patienten mit mäßig aktiver Colitis ulcerosa, die zuvor nicht auf Kortikosteroide und/oder Immunmodulatoren angesprochen hatte, behandelt. $69 \%$ der Patienten, die eine Dosis von $5 \mathrm{mg} / \mathrm{kg}$ KG erhielten, zeigten in Woche 8 ein klinisches Ansprechen, in der Gruppe der Patienten, die $10 \mathrm{mg} / \mathrm{kg}$ KG erhielten, waren es $61 \%$ und in der Plazebogruppe $37 \%$; die Remissionsraten lagen bei $38,8 \%$ (5 mg/kg KG Infliximab), $32 \%$ (10 mg/kg KG Infliximab) und $14,9 \%$ (Plazebo). Die Gabe von Infliximab kann dann in einer Dosierung von $5 \mathrm{mg} / \mathrm{kg} \mathrm{KG}$ zu den Zeitpunkten Woche 0, 2 und 6 erfolgen. Eine Fortführung in 8-wöchigen Abständen als Erhaltungstherapie sollte sich, bei entsprechender Wirksamkeit, anschließen [350].

Für die steroidrefraktäre Situation liegt zum einen die doppelblinde, randomisierte Pilotstudie von Järnerot und Kollegen vor, die 45 Patienten mit schwerer Colitis ulcerosa eingeschlossen hat, die zuvor nicht auf die konventionelle Steroidtherapie angesprochen hatten [379]. Sieben Patienten der Infliximab-Gruppe und 14 Patienten der Plazebogruppe mussten sich innerhalb von 3 Monaten nach Randomisierung einer Proktokolektomie unterziehen (statistisch signifikanter Unterschied). Die Proktokolektomierate in diesem Kollektiv betrug nach drei Jahren 12/24 (50\%) Patienten in der Infliximab-Gruppe sowie 16/21 in der PlazeboGruppe [380]. In einer retrospektiven Multicenterstudie mit 211 Patienten mit steroidrefraktärer Colitis, die Infliximab erhielten, betrugen die Proktokolektomieraten 36\%, $41 \%$ und $46 \%$ nach 1, 3 und 5 Jahren [381]. Auf die französische Studie sowie die CONSTRUCT-Studie und damit die beiden größten kontrollierten Studie für diese Fragestellung wurde oben bereits eingegangen und gezeigt, dass Infliximab dem Ciclosporin nicht unterlegen ist [366, 367]. Die Kolektomieraten für die mit Infliximab behandelten Patienten betrugen in der CONSTRUCT-Studie $21 \%$ während des initialen Krankenhausaufenthalts, $29 \%$ in 3 Monaten und $35 \%$ nach 12 Monaten [367]. 
Welche Faktoren dienen uns als Prädiktoren? Bestehen bei Aufnahme eines steroidrefraktären Patienten eine deutliche CRPErhöhung, niedrige Serumalbuminspiegel, Seropositivität für perinukleäre antineutrophile zytoplasmatische Antikörper sowie schwere endoskopische Läsionen, dann ist die Wahrscheinlichkeit für ein kurzfristiges Rezidiv oder eine Proktokolektomie hoch [382, 383]. Für eine proktokolektomiefreie Remissionserhaltung sprechen ein schnelles Ansprechen, mukosale Heilung und Infliximab-Serumspiegel $>2,5 \mu \mathrm{g} / \mathrm{ml}$ in Woche 14. Damit übereinstimmend sprechen niedrige Serumspiegel in Woche $6(<2,5 \mu \mathrm{g} / \mathrm{ml})$ für ein primäres Nichtansprechen [384]. Warum sind die Serumspiegel in den Fällen der schweren Colitis ulcerosa niedrig? Es konnte gezeigt werden, dass bei ihnen Infliximab über den Stuhl verloren geht [385]. Auch wenn die Induktion in der CONSTRUCT-Studie mit einer Gabe in den Wochen 0, 2 und 6 den Standard darstellt, weist eine kleine retrospektive Studie darauf hin, dass vielleicht ein beschleunigtes Induktionsschema mit einer Reduktion der frühen Proktokolektomierate in 50 hospitalisierten Patienten assoziiert ist [386]. Zusätzlich hatten thiopurinnaive Patienten einen besseren Verlauf in Bezug auf eine Operation [387].

\section{Abwägung Infliximab versus Calcineurininhibitor}

Die CYSIF-Studie randomisierte 111 thiopurinnaive Patienten mit schwerer Colitis ulcerosa (Lichtiger Score $>10$ ) trotz erfolgter fünftägiger intravenöser Steroidtherapie [366]. Die Patienten erhielten entweder Ciclosporin ( $2 \mathrm{mg} / \mathrm{kg}$ KG für 8 Tage, Zielspiegel: $150-250 \mu \mathrm{g} / \mathrm{ml}$; gefolgt von $4 \mathrm{mg} / \mathrm{kg} \mathrm{KG}$ Ciclosporin oral) oder Infliximab (5 mg/kg KG in den Wochen 0, 2 und 6) [366]. Alle Patienten, die ein Ansprechen an Tag 8 zeigten, erhielten orales Azathioprin und begannen die Steroide zu reduzieren. Die Studie war angelegt, um zu zeigen, dass unter Ciclosporin weniger Therapieversager auftreten. Etwa 85 \% der Patienten in beiden Gruppen zeigten jedoch an Tag 7 ein Therapieansprechen. Ein Therapieversagen an Tag 98 zeigte sich bei $60 \%$ in der Ciclosporinund bei $54 \%$ in der Infliximab-Gruppe (nicht signifikant). Die Proktokolektomierate an Tag 98 betrug $18 \%$ in der Ciclosporin- und $21 \%$ in der Infliximab-Gruppe (nicht signifikant). Ebenfalls zeigten sich keine Unterschiede bei den schweren Nebenwirkungen. Auch die oben bereits diskutierte CONSTRUCT-Studie, die als primäres Ziel hatte zu zeigen, dass Infliximab dem Ciclosporin nicht unterlegen ist, konnte keine signifikanten Unterschiede zwischen den beiden Behandlungsarmen im Hinblick auf Lebensqualität, Proktokolektomie, Mortalität oder das Auftreten schwerer Infektionen zeigen [367]. Damit übereinstimmend zeigte eine Metaanalyse, die sechs retrospektive Arbeiten einschloss, vergleichbare Remissionsraten bei Patienten mit einer schweren, steroidrefraktären Colitis ulcerosa, die entweder Ciclosporin oder Infliximab erhielten [388]. Aus ökonomischer Sicht konnten die Zeit und die Kosten des stationären Aufenthalts gesenkt werden, jedoch stiegen gleichzeitig die gesamten Behandlungskosten an [389].

Damit kann die Frage, welche der beiden Substanzen eingesetzt werden sollte, nicht eindeutig beantwortet werden. Dies stellt eher eine individuelle Entscheidung dar. Bei Patienten mit einem niedrigen Cholesterin- und Magnesiumspiegel sollte Ciclosporin aufgrund des erhöhten Risikos neurologischer Komplikationen eher vermieden werden. Bei Ansprechen auf die Infliximab-
Therapie ist der Übergang in die Erhaltungstherapie in der Praxis einfacher. Lange war das Argument, lieber Ciclosporin einzusetzen, da, sollte es zur Operation kommen, mit weniger Komplikationen zu rechnen sei [390]. Die Daten beruhen jedoch auf einer kleinen Fallserie und die Daten für Infliximab erlauben derzeit keine eindeutige Aussage, womit aus ihnen keine sichere Schlussfolgerung gezogen werden kann. Viel entscheidender für das operative Risiko ist die langfristige Einnahme von Steroiden [391].

Die weitere Frage ist, wie medikamentös vorgegangen wird, wenn es nach Steroiden entweder mit Infliximab oder einem Calcineurininhibitor zu einem Therapieversagen kommen sollte. Ist es sinnvoll, in dieser Situation die jeweils andere Option zu versuchen? Zunächst ist dies in jedem Fall eine Situation, in der man die chirurgischen Optionen ausführlich besprechen und anbieten sollte. Es liegen keine kontrollierten Daten für diese Situation vor, sondern nur Fallserien, die nicht hilfreich sind [392]. Eine Drittlinientherapie sollte daher, wenn überhaupt, spezialisierten Zentren vorbehalten sein. In diesem Fall sollte eine enge Abstimmung mit dem chirurgischen Partner erfolgen.

\section{Komplikationen}

Auf die im akuten Schub vermehrt auftretenden thromboembolischen Ereignisse wurde oben bereits eingegangen; eine prophylaktische Antikoagulation stellt für sie den Standard dar [341, 343]. Die Perforation im akuten Schub, die spontan auftreten oder auch die Folge einer endoskopischen Untersuchung sein kann, ist mit einer Mortalität von bis zu $50 \%$ assoziiert [323]. Eine weitere Komplikation stellt die massive Blutung dar.

Von herausragender Bedeutung bei der Behandlung dieser schwer kranken Patienten ist es, nicht den Zeitpunkt zu versäumen, an dem die konventionelle/medikamentöse Therapie versagt und eine chirurgische Therapie (Proktokolektomie) notwendig wird. Mit der zunehmenden Zahl an Therapiealternativen nimmt die Gefahr der Verzögerung der notwendigen chirurgischen Therapie tendenziell weiter zu. Aus diesem Dilemma ergibt sich, dass Gastroenterologen/Kindergastroenterologen und Chirurgen sehr eng miteinander kooperieren und ständig gemeinsam den Zustand der Patienten und das Ansprechen auf die Therapie beurteilen müssen. Eine intensive Überwachung der Patienten ist dabei unerlässlich.

\section{Sondersituation refraktäre Proktitis}

Die refraktäre Proktitis stellt häufig eine klinische Herausforderung dar. Zunächst müssen die Differenzialdiagnosen kritisch überprüft werden (HIV-, Chlamydieninfektion, Reizdarmsyndrom, anatomische Besonderheit des Beckenbodens (Prolaps), Morbus Crohn, Karzinom). Im zweiten Schritt muss überprüft werden, ob der Patient die verordneten Medikamente auch einnimmt bzw. appliziert, und eine verlangsamte Darmmotilität sollte ausgeschlossen werden [393]. Die Therapie erfolgt dann, wie oben für die klassische refraktäre Situation dargestellt, auch wenn kaum Studien exklusiv die Proktitis im Fokus haben. Darüber hinaus liegen kleine Studien vor, die den Einsatz von lokalen Calcineurininhibitoren (als Einlauf oder Suppositorien) favorisieren [377, 394 397]. Des Weiteren liegt eine Reihe unkontrollierter Studien vor, die keine allgemeine Schlussfolgerung erlauben. Kritisch bleibt 
festzuhalten, dass bis zu $10 \%$ der Patienten, die aufgrund einer therapierefraktären Colitis eine Koloproktektomie erhalten, nur an einer distalen Colitis leiden.

\section{EMPFEHLUNG 3.29}

Nach Ansprechen auf eine Therapie mit Calcineurininhibitoren kann eine Therapie mit Azathioprin/Mercaptopurin (Evidenzgrad 3, Empfehlungsgrad 0) oder Vedolizumab (Evidenzgrad 4, Empfehlungsgrad 0) eingeleitet werden. Bei Ansprechen auf eine Therapie mit TNF-Antikörpern soll diese Therapie zur Remissionserhaltung fortgesetzt werden (Evidenzgrad 1, Empfehlungsgrad A).

Konsens

\section{Hintergrund}

Aufgrund der hohen Kolektomierate bei Patienten, die wegen eines steroidrefraktären Verlaufs eine Therapie mit Cyclosporin oder Tacrolimus erhielten, empfiehlt sich selbst bei 5-ASA-naiven Patienten die Durchführung einer remissionserhaltenden Therapie [363, 370, 398, 399]. Da Calcineurininhibitoren aufgrund der Nebenwirkungsraten nach spätestens 6 Monaten abgesetzt werden sollten, sollten zur Remissionserhaltung andere Substanzen, beispielsweise Thiopurine, eingesetzt werden. Retrospektive Analysen belegen, dass Thiopurine das Risiko einer Kolektomie nach Induktion mit Ciclosporin reduzieren [372, 399]. Insbesondere bei thiopurinnaiven Patienten empfiehlt sich ein überlappender Therapiebeginn mit Thiopurinen, sobald ein Ansprechen bzw. eine Remission mit einem Calcineurininhibitor erreicht wurde. Nach einer intravenösen Remissionsinduktion mit Ciclosporin kann eine überbrückende Therapie mit oralem CsA bis zur Erreichung des therapeutischen Effekts von Azathioprin durchgeführt werden.

Neuerdings wird bei Patienten, die einen azathioprinrefraktären Verlauf aufweisen, nach erfolgreicher Remissionsinduktion mit Calcineurininhibitoren auch eine Erhaltungstherapie mit Vedolizumab vorgeschlagen [400]. Die remissionserhaltende Therapie mit TNF-Antikörpern nach entsprechender Remissionsinduktion wird unter der Empfehlung 3.34 ausführlich diskutiert.

\section{Colitis ulcerosa mit steroidabhängigem Verlauf}

\section{EMPFEHLUNG 3.30}

Patienten mit einer steroidabhängigen Colitis ulcerosa sollten mit einem Thiopurin (Evidenzgrad 2) oder TNF-Antikörpern (Evidenzgrad 1) (im Fall von Infliximab ggf. kombiniert mit einem Thiopurin (Evidenzgrad 2)) oder mit Vedolizumab (Evidenzgrad 2) behandelt werden. Empfehlungsgrad B, starker Konsens

\section{Hintergrund}

Die Wirksamkeit von Thiopurinen, TNF-Antikörpern und Vedolizumab bei der steroidabhängigen Verlaufsform einer Colitis ulcerosa wurde in verschiedenen Studien untersucht. Eine generelle Empfehlung bezüglich der Wahl der geeigneten Therapie bei Steroidabhängigkeit kann aufgrund fehlender Vergleichsstudien nicht gegeben werden. Die Wahl des Therapeutikums ist u.a. abhängig von der Aktivität der Erkrankung, dem Alter und den Komorbiditäten des Patienten, dem potenziellen Nebenwirkungsprofil, dem klinischen Remissionsdruck, den Kosten sowie dem Patientenwunsch. Bei steroidabhängiger Colitis ulcerosa ist Azathioprin signifikant wirksamer als 5-ASA zur Induktion einer klinischen und endoskopischen Remission. In einer Open-Label-Studie wurden 72 Patienten zwischen einer Therapie mit Azathioprin $2 \mathrm{mg} / \mathrm{kg} / \mathrm{d}$ und oralem 5-ASA 3,2 g/d ergänzend zu einer Steroidtherapie mit $40 \mathrm{mg} / \mathrm{d}$ Prednisolon randomisiert [401]. $53 \%$ der Patienten unter Azathioprin erreichten eine steroidfreie klinische und endoskopische Remission nach 6 Monaten, verglichen mit $21 \%$ der mit 5-ASA behandelten Patienten. In einer Open-LabelKohortenstudie mit 42 Patienten unter Azathioprin-Therapie ergaben sich steroidfreie Remissionsraten von 55\%, $52 \%$ und $45 \%$ nach 12, 23 und 36 Monaten [402]. Aus diesen Studien lässt sich eine effektive Wirkung von Thiopurinen bei Patienten mit steroidabhängiger Colitis ulcerosa ableiten.

Steroidfreie Remission war ein sekundärer Endpunkt der großen klinischen Anti-TNF-Studien. Eine klare Differenzierung zwischen steroidabhängigem und steroidrefraktärem Verlauf wurde in keiner dieser Studien durchgeführt. Es liegen lediglich Daten dazu vor, wie hoch der Anteil der Patienten war, die unter dieser Therapie Steroide erhielten. Bezüglich Infliximab untersuchten die ACT-1- und die ACT-2-Studie 364 Patienten mit endoskopisch gesicherter Colitis ulcerosa, bei denen trotz Therapie mit Kortikosteroiden und/oder Thiopurinen (ACT-1) und Kortikosteroiden und/oder Thiopurinen und/oder 5-ASA (ACT-2) eine mittelschwere bis schwere Krankheitsaktivität vorlag. Alle Patienten waren anti-TNF-naiv und wurden zwischen Plazebo und Infliximab randomisiert. $56 \%$ der Patienten nahmen bei Studieneinschluss Kortikosteroide, 38 \% mit einer Äquivalenzdosis von mindestens $20 \mathrm{mg} / \mathrm{d}$ Prednisolon. Von den Patienten, die zur Baseline Steroide einnahmen, erreichten unter Infliximab 21,5\% eine steroidfreie Remission zur Woche 30, unter Plazebo 7,2\% ( $p=0,007)$ [350].

Die ULTRA-2-Studie untersuchte die Wirkung von Adalimumab bei 494 Patienten mit endoskopisch gesicherter Colitis ulcerosa mit mittelstarker bis starker Krankheitsaktivität trotz Therapie mit Kortikosteroiden und/oder Thiopurinen. Die Patienten wurden zwischen Plazebo und einer Therapie mit $160 \mathrm{mg}$ Adalimumab an Woche $0,80 \mathrm{mg}$ an Woche 2 und dann $40 \mathrm{mg}$ zweiwöchentlich randomisiert [351]. 59\% der Patienten nahmen zur Baseline Steroide, $40 \%$ waren im Vorfeld TNF-Versager, $31 \%$ der Patienten waren zur Woche 16 steroidfrei, aber nur $16 \%$ unter Plazebo $(p<0,05)$. Zur Woche 52 waren unter Adalimumab 13,3\% der Patienten, die unter Steroiden in die Studie eingeschlossen wurden, in steroidfreier Remission, unter Plazebo nur 5,7\% $(p=0,035)$.

Die Wirksamkeit von Golimumab bei Patienten, bei denen trotz Therapie mit Steroiden oder 5-ASA oder Thiopurinen eine mittelschwere bis schwere Colitis ulcerosa vorlag, wurde in den 
PURSUIT-Studien belegt [352, 353]. Alle Studienteilnehmer waren anti-TNF-naiv. In diesen Studien war die Induktionsphase von der Remissionserhaltungsphase getrennt. Das klinische Ansprechen lag zu Woche 6 bei $51,0 \%$ bei Patienten, die mit 200 und dann $100 \mathrm{mg}$ Golimumab behandelt worden waren, bzw. 5,9\% bei Patienten, die mit $400 \mathrm{mg}$ und dann $200 \mathrm{mg}$ behandelt worden waren [352]. In der PURSUIT-M-Studie wurden 464 Patienten, die in der Induktionsphase ein Ansprechen gezeigt hatten, erneut zur Erhaltungstherapie mit Golimumab oder Plazebo randomisiert. In der Subgruppe der Patienten, die zur Baseline Steroide einnahmen, waren zur Woche 54 34,4\% unter Golimumab in steroidfreier Remission, unter Plazebo 20,7\% ( $p=0,024)$ [353].

Die genannten TNF-Antikörper sind wirksamer als Plazebo in der Induktion und dem Erhalt einer steroidfreien Remission bei Patienten unter Steroidtherapie zur Baseline.

Die Frage des Nutzens einer Kombinationstherapie von Infliximab mit Azathioprin im Vergleich mit Infliximab allein bzw. Azathioprin allein wurde in der UC-SUCCESS-Studie an biologikanaiven und mehrheitlich thiopurinnaiven Patienten untersucht [403]. In dieser randomisiert kontrollieren Studie stellte sich die Kombinationstherapie von Infliximab und Azathioprin wirksamer als die Monotherapien dar; unter der kombinierten Therapie erreichten 39,7 \% der Patienten in Woche 16 eine steroidfreie Remission, unter Infliximab 22,1\% und unter Azathioprin 23,7\% ( $p=0,032)$.

Entsprechende Daten zur Wirksamkeit einer Kombinationstherapie bei Colitis ulcerosa liegen für Adalimumab und Golimumab nicht vor. Für diese Substanzen gibt es lediglich Hinweise auf eine Reduktion einer Autoantikörperbildung durch eine Ko-Immunsuppression. Ein Beleg dafür, dass dies jedoch auch die Wirksamkeit verbessert, steht noch aus.

Steroidfreie Remission war ebenso ein sekundärer Endpunkt bei der GEMINI-1-Studie. Bei dieser Studie zur Wirkung von Vedolizumab bei mittelschwerer bis schwerer Colitis ulcerosa erfolgte eine randomisierte Induktionsphase mit 374 Patienten. Die Responder in Woche 6 wurden dann zwischen Vedolizumab oder Plazebo für die Erhaltungstherapie erneut randomisiert [404]. In einem weiteren Induktionsarm wurden Patienten aus einer Open-Label-Kohorte, die auf Vedolizumab angesprochen hatten, für die Remissionserhaltung zwischen Vedolizumab und Plazebo randomisiert. Die Studienteilnehmer waren refraktär auf Steroide und/oder Thiopurine oder eine Anti-TNF-Therapie.

53,7\% der Patienten der GEMINI-1-Studie standen unter einer Steroidtherapie zum Studieneinschluss, 48 \% waren Versager einer Anti-TNF-Therapie. Von den Patienten unter Steroidtherapie bei Studieneinschluss, die auf die Induktionstherapie angesprochen hatten, waren 38,5\% der auf eine Remissionserhaltung mit Vedolizumab randomisierten Patienten in Woche 52 in steroidfreier Remission, verglichen mit 13,9\% unter Plazebo $(p<0,001)$. Die Effektivität der Therapie mit Vedolizumab war sowohl in der Induktions- als auch in der Erhaltungstherapie unabhängig von einer Begleittherapie mit Steroiden oder Immunsuppressiva und ebenso unabhängig von einer vorangegangenen Anti-TNFTherapie.

Methotrexat (MTX) wurde kürzlich in einer multizentrischen Studie an 111 Patienten mit steroidabhängiger Colitis ulcerosa untersucht [405]. Der primäre Endpunkt mit einer steroidfreien Remission in Woche 16 bei 31,7\% der Patienten unter MTX im
Vergleich zu 19,6\% ( $p=0,15)$ unter Plazebo wurde nicht erreicht. Der sekundäre Endpunkt mit dem Anteil der Patienten mit steroidfreier klinischer Remission in Woche 16 lag unter MTX bei $41,7 \%$ und unter Plazebo bei 23,5\% ( $p=0,04)$. Wenngleich ein klarer Trend bezüglich eines Therapieansprechens erkennbar ist, kann aufgrund der aktuellen Datenlage keine generelle Empfehlung zum Einsatz von Methotrexat bei Colitis ulcerosa ausgesprochen werden.

\section{Colitis ulcerosa mit nicht ausreichendem Ansprechen auf eine Therapie mit Thiopurinen}

\section{EMPFEHLUNG 3.31}

Patienten mit leichter bis mittelschwerer Colitis ulcerosa, die nicht ausreichend auf eine Therapie mit Thiopurinen ansprechen, sollten mit TNF-Antikörpern (Evidenzgrad 1) (im Fall von Infliximab ggf. in Kombination mit Thiopurinen (Evidenzgrad 2)) oder mit Vedolizumab (Evidenzgrad 2) behandelt werden.

Empfehlungsgrad B, starker Konsens

\section{Hintergrund}

Nach Ausschluss infektiöser Komplikationen sollte bei Versagen einer immunsuppressiven Therapie eine Behandlung mit Biologika erfolgen, sofern diesbezüglich keine Kontraindikationen vorliegen. Grundsätzlich kann eine Therapie mit Integrin-Antikörpern auch schon vor einem Einsatz mit TNF-Antikörpern erfolgen. Infliximab, Adalimumab, Golimumab und Vedolizumab wurden sämtlich in Studien zur Behandlung der CU bei Patienten, die refraktär auf Thiopurine waren, untersucht.

Die ACT-1- und die ACT-2-Studie haben 334/728 (46\%) Patienten mit aktiver Erkrankung trotz immunsuppressiver Therapie eingeschlossen [350]. Unter Infliximab erreichten unabhängig von der Dosis (5 oder $10 \mathrm{mg}$ ) signifikant mehr Patienten nach 8 Wochen eine klinische Remission als unter Plazebo. Dabei wurde allerdings das Ansprechen in der Subgruppe von Patienten, die immunsuppressivarefraktär waren, nicht berichtet. In einer Cochrane Database Review wurden 7 Studien von Infliximab bei Patienten mit mittelschwerer bis schwerer Colitis ulcerosa ausgewertet, die refraktär auf eine Steroidtherapie und/oder Immunsuppression waren [406]. Drei Infusionen (Woche 0, 2 und 6) waren bei der Induktion einer klinischen Remission in Woche 8 effektiver als Plazebo (RR 3,22, 95 \%-KI 2,18-4,76). Auch in diesem Fall wurde keine Subgruppenanalyse für Patienten durchgeführt, die refraktär auf eine Therapie mit Immunsuppressiva waren.

In der ULTRA-1-Studie wurde eine Überlegenheit von Adalimumab gegenüber Plazebo bei der Remissionsinduktion bei Patienten mit CU berichtet. 159 von 390 Patienten (39\%) erhielten eine Immunsuppression zur Baseline [407, 408]. Bei Patienten mit begleitender Immunsuppression konnte Adalimumab in Woche 8 bei 15,1\% (8/53) eine Remission induzieren, unter Plazebo bei $0 \%$ (0/15); bei Patienten unter Immunsuppression und Steroidtherapie zur Baseline lagen die Remissionsraten in Woche 8 bei 12,2\% (6/49) unter Adalimumab und 9,5\% (2/34) unter Plazebo. In der ULTRA-2-Studie waren 173 von 494 Patienten unter immunsup- 
pressiver Therapie [351]. Adalimumab $160 \mathrm{mg} / 80 \mathrm{mg} / 40 \mathrm{mg}$ zweiwöchentlich induzierte eine klinische Remission in Woche 8 bei 8/53 (15,1\%) Patienten verglichen mit 2/52 (3,8\%) Patienten unter Plazebo.

Eine prospektive Kohortenstudie untersuchte 53 Patienten unter Infliximab oder Adalimumab bei mittelschwerer CU. Die klinische Ansprechrate lag bei 88,7\% ohne signifikanten Unterschied zwischen den Therapiegruppen [409]. Alle eingeschlossenen Patienten waren intolerant oder Therapieversager auf eine immunsuppressive Therapie, obwohl nur 5/25 Patienten unter Adalimumab und 15/28 Patienten unter Infliximab zur Baseline eine immunsuppressive Therapie erhalten hatten.

In der PURSUIT-Studie mit Golimumab wurden 31,2\% der Patienten mit mittelschwerer bis schwerer Krankheitsaktivität mit Thiopurinen behandelt. Die begleitende Immunsuppression beeinflusste das Outcome nicht.

Bei Patienten, die refraktär auf eine immunsuppressive Therapie sind, stellt sich häufig die Frage, ob die Immunsuppression bei Beginn einer Anti-TNF-Therapie fortgeführt werden soll. In die UC-SUCCESS-Studie wurden nur Patienten mit steroidrefraktärem Verlauf eingeschlossen. Die Patienten mussten entweder naiv auf Immunsuppressiva sein (90\%) oder mindestens drei Monate vor Baseline keine Immunsuppression erhalten haben. Wegen des hohen Anteils von immunsuppressivanaiven Patienten lassen sich die Daten vermutlich nicht direkt auf immunsuppressivarefraktäre Patienten extrapolieren. Die Empfehlung zur begleitenden Immunsuppression bei Infliximab-Therapie beruht daher auf indirekten Daten, die zeigen, dass eine begleitende Immunsuppression die Bildung von Autoantikörpern reduzieren kann und/oder durch höhere Through Level die Effektivität der Therapie erhöht [403, 410]. Weder Subgruppenanalysen klinischer Studien noch retrospektive Analysen pharmakokinetischer Proben ergaben ähnliche Ergebnisse für Adalimumab oder Golimumab [353, 411]. Hinweise auf eine vermehrte klinische Wirksamkeit einer Co-Immunsuppression gibt es für Adalimumab und Golimumab jedoch nicht.

In die GEMINI-1-Studie wurden 17,8\% Patienten unter Immunsuppression und 16,6\% unter Immunsuppression und Steroiden eingeschlossen [404]. Die Subgruppenanalyse zum Ansprechen auf die Induktionstherapie enthielt nicht die gesamte Gruppe der Immunsuppressionsversager, sondern nur die von Versagern auf eine Immunsuppression, die im Vorfeld kein Versagen auf eine Anti-TNF-Therapie gezeigt hatten. In dieser Gruppe wurde ein Trend zum Nutzen von Vedolizumab gegenüber Plazebo beobachtet, der aber keine Signifikanz erreichte ( $49 \%$ vs. $34,5 \%, p=0,08$ ). Die gleiche Subgruppenanalyse in der Erhaltungstherapie ergab 1 Jahr nach erneuter Randomisierung von Patienten, die auf die Induktionstherapie angesprochen hatten eine signifikant höhere Remissionsrate unter Vedolizumab verglichen mit Plazebo (44,6\% unter Vedolizumab alle 8 Wochen, $50 \%$ unter Vedolizumab alle 4 Wochen und $18 \%$ unter Plazebo). Eine begleitende immunsuppressive Therapie hat diese Ergebnisse nicht wesentlich beeinflusst. Eine Entscheidung zu einer Therapie mit Vedolizumab sollte in dieser Situation von der Krankheitsaktivität der CU abhängig gemacht werden, da mit einem Therapieansprechen erst nach einigen Wochen gerechnet werden kann.
Es liegen nur wenige Daten zur Wirkung einer zweiten AntiTNF-Substanz nach Versagen einer Anti-TNF-Therapie vor. Bezüglich Vedolizumab wurden in die GEMINI-1-Studie $48 \%$ Patienten mit vorangegangenem Versagen einer Anti-TNF-Therapie eingeschlossen. In Subanalysen gab es keine signifikanten Unterschiede dieser Subgruppe zur gesamten Studienpopulation, was nahelegt, dass Patienten mit vorangegangenem Anti-TNF-Versagen ein ähnliches Outcome haben wie anti-TNF-naive Patienten.

In die OCTAVE-1- und OCTAVE-2-Studie zur Wirksamkeit von Tofacitinib bei $\mathrm{CU}$ wurden Patienten mit vorangegangenem Therapieversagen auf Azathioprin eingeschlossen. Damit könnte Tofacitinib eine Option für Patienten mit mittelschwerer bis schwerer CU sein, die refraktär auf eine Therapie mit Thiopurinen sind. Da noch keine Zulassung und Verfügbarkeit von Tofacitinib zur Behandlung der CU in Deutschland vorliegt, wurde diese Substanz zum Redaktionsschluss noch nicht mit in die Empfehlungen der Leitlinie aufgenommen.

Neben einigen Fallserien, die den Einsatz von Tacrolimus bei CU unterstützen [248, 412], gibt es keine kontrollierten klinischen Studien zu dieser Therapie bei Patienten mit Versagen einer immunsuppressiven Therapie.

Mit den Patienten sollten die Vorzüge und Risiken einer kombinierten immunsuppressiven Therapie auch im Vergleich zur Option einer operativen Therapie kritisch besprochen werden.

\section{Colitis ulcerosa mit primärem oder sekundärem Therapie- versagen unter einer Therapie mit TNF-Antikörpern}

EMPFEHLUNG 3.32

Patienten mit primärem Versagen auf eine Therapie mit TNFAntikörpern sollten mit Vedolizumab (Evidenzgrad 2, Empfehlungsgrad B) oder Calcineurininhibitoren (Evidenzgrad 3, Empfehlungsgrad B) behandelt werden. Patienten mit mittelschwerer Colitis ulcerosa mit sekundärem Versagen auf eine Therapie mit TNF-Antikörpern sollten mit alternativen TNFAntikörpern (Evidenzgrad 4, Empfehlungsgrad B) oder Vedolizumab (Evidenzgrad 2, Empfehlungsgrad B) oder Calcineurininhibitoren (Evidenzgrad 3, Empfehlungsgrad B) behandelt werden. Eine Proktokolektomie sollte in Erwägung gezogen werden. (Expertenkonsens, Empfehlung).

Starker Konsens

\section{Hintergrund}

Bei der Frage, welche Therapieoptionen bei einem Therapieversagen auf TNF-Antikörper zur Verfügung stehen, ist die Differenzierung zwischen primärem Nichtansprechen oder einem sekundären Therapieversagen von klinischer Bedeutung. Grundsätzlich sollte auch bei primärem Therapieversagen eines TNF-Antagonisten die Möglichkeit einer Therapieintensivierung durch Dosiserhöhung in Betracht gezogen werden. Insbesondere bei endoskopisch starker Entzündung kann durch den sogenannten „fecal loss“ applizierter Antikörper über den Darm verloren gehen und eine Dosiserhöhung notwendig sein [385]. Bei einem primären Therapieversagen hängt die Wahl der weiteren Therapie ansonsten entscheidend von dem Remissionsdruck ab. Bei hoher 
Krankheitsaktivität würde eher die Entscheidung zugunsten des Einsatzes eines Calcineurininhibitors fallen, da dann eine rasche Remissionsinduktion zu erwarten ist. Bei geringradiger Entzündungsaktivität mit geringerem Remissionsdruck kann auch der Einsatz von Vedolizumab in Erwägung gezogen werden. Der Wechsel von einem TNF-Antikörper auf einen anderen TNF-Antikörper ist bei primärem Therapieversagen nur mit einer geringen Aussicht auf Erfolg in etwa $30 \%$ der Fälle verbunden [413], sodass dazu nicht geraten werden kann.

Anders stellt sich die Situation bei sekundärem Therapieversagen dar. In der ULTRA-2-Studie wurde der co-primäre Endpunkt einer klinischen Remission in Woche 16 in der Subgruppe von Patienten mit Anti-TNF-Versagen im Vorfeld nicht erreicht; der weitere klinische co-primäre Endpunkt einer klinischen Remission in Woche 52 wurde erreicht (10,2\% unter Adalimumab vs. 3,0\% unter Plazebo, $p=0,039)$. Der Unterschied war bei diesem und anderen Endpunkten geringer als bei anti-TNF-naiven Patienten. Der sekundäre Endpunkt einer steroidfreien Remission in Woche 52 unter den Patienten, die zur Baseline unter Steroiden standen, wurde unter den Anti-TNF-Versagern nicht erreicht. In einer Metaanalyse wurden 8 Studien, bei denen das Ansprechen auf eine Zweitlinientherapie mit einem TNF-Antikörper nach sekundärem Versagen einer primären TNF-Antikörper-Therapie untersucht wurde, ausgewertet. In allen Studien wurde von Infliximab auf Adalimumab gewechselt, die Ansprechraten lagen dabei zwischen $23 \%$ und $92 \%$, die Remissionsraten zwischen $0 \%$ und $50 \%$ [413]. Wenngleich die Studien sehr heterogen sind und keine gepoolte Datenanalyse erlauben, rechtfertigt die derzeitige Datenlage doch einen versuchsweisen Wechsel von einem auf einen zweiten TNF-Antikörper.

In der GEMINI-1-Studie, in der $48 \%$ der Patienten Anti-TNF-Versager waren, war die Remissionsrate unabhängig von einer vorangegangenen Therapie mit TNF-Antikörpern [404]. In einer deutsche Kohortenstudie waren $25 \%$ der Patienten mit Colitis ulcerosa oder Morbus Crohn nach 14 Wochen in klinischer Remission [414]. Daten zur Wirksamkeit von TNF-Antikörpern nach primärem Therapieversagen von Vedolizumab liegen derzeit nicht vor.

Zwei Phase-3-Studien haben die Wirkung des JAK-Inhibitors Tofacitinib (10 mg zweimal täglich) als Induktionstherapie an insgesamt 1139 Patienten mit mittelschwerer bis schwerer CU untersucht [415]. Die eingeschlossenen Patienten hatten ein Therapieversagen auf Steroide, Azathioprin oder Anti-TNF-Therapie gezeigt (53-58\% der Patienten hatten zuvor eine der drei genannten Therapien). Eine Remission an Woche 8 erreichten 18,5\% und 16,6\% unter Tofacitinib und 8,2 bzw. 3,6\% unter Plazebo. Beide Unterschiede waren statistisch signifikant. Unter Tofacitinib fielen erhöhte Cholesterin- und Kreatininwerte und in der 10-mg-Gruppe auch das vermehrte Auftreten von Infektionen, insbesondere Herpesinfektionen, auf. Tofacitinib ist zum Redaktionsschluss in Deutschland noch nicht zur Therapie der Colitis ulcerosa zugelassen, sodass bei fehlenden klinischen Daten die genaue Einordnung in den Therapiealgorithmus der CU noch nicht abschließend beurteilt werden kann.

Ozanimod, ein Modulator der Sphingosin-1-Phosphate-Rezeptor-Subtypen 1 und 5, wurde in einer randomisierten Phase-2-Studie untersucht. Dabei erreichten 16\% der Patienten unter Ozanimod eine klinische Remission in Woche 8, unter Plazebo $6 \%$ $(p=0,048)$ [416]. Zur weiteren Bewertung der Wirksamkeit und Sicherheit von Ozanimod sind noch weitere Studien nötig.

Da es sich bei den Patienten mit primärem oder sekundärem Therapieversagen auf TNF-Antikörper grundsätzlich um eine sehr kranke und häufig „austherapierte“ Patientenpopulation handelt, sollte in dieser Situation die Möglichkeit einer Proktokolektomie immer mit in Erwägung gezogen und mit den Patienten diskutiert werden.

\section{Anwendung von Biosimilars bei Colitis ulcerosa}

Zum Zeitpunkt der Leitlinienerstellung steht für die Anwendung bei Colitis ulcerosa lediglich das Infliximab-Biosimilar zur Verfügung. Weitere Biosimilars werden jedoch in den kommenden Jahren folgen. Eine generelle Empfehlung zur Anwendung von Biosimilars wird in dieser Leitlinie nicht gegeben, da es sich bei Biosimilars lediglich um andere Versionen eines monoklonalen Antikörpers bzw. derzeit um Infliximab handelt, deren Anwendungen an verschiedenen anderen Stellen besprochen werden. Da die Anwendung der Biosimilars derzeit viel und z. T. kontrovers diskutiert wird, folgen zumindest einige generelle Überlegungen zur Anwendung von Biosimilars.

Die molekulare Struktur der zurzeit verfügbaren InfliximabBiosimilars ist der des Referenzprodukts sehr ähnlich. Alle Medikamente haben ähnliche physikalische und chemische Eigenschaften, biologische Aktivität, Pharmakokinetik und Toxizität in Tierversuchen wie bei gesunden Probanden. Zwei Phase-3-Studien haben zwischen Biosimilar und Referenzprodukt eine vergleichbare Effektivität, Toxizität und Immunogenität bei Patienten mit Rheumatoider Arthritis und Ankylosierender Spondylarthritis gezeigt [417, 418]. Mehrere Open-Label-Studien legen nahe, dass das Infliximab-Biosimilar bei CU wirksam ist. Auf der Basis vorklinischer und klinischer Daten hat die EMA den Einsatz der Infliximab-Biosimilars bei rheumatoider Arthritis, Spondylarthritis, $\mathrm{CU}$ und Morbus Crohn zugelassen. Es ist zu erwarten, dass durch die in den kommenden Jahren vermehrt auf dem Markt erscheinenden bioähnlichen Substanzen das Spektrum der Anwendung von Biosimilars bei Colitis ulcerosa deutlich erweitert werden wird. Die Verfügbarkeit von Biosimilars ist wettbewerbsfördernd und wird zur Kostensenkung in einem finanziell überstrapazierten Gesundheitssystem beitragen. Außerdem werden damit wichtige Medikamente für eine breitere Gruppe von Patienten verfügbar gemacht. Biosimilars scheinen daher eine wirkliche Alternative zu den Originalprodukten zu sein. Trotz fehlender randomisierter Studien im Indikationsbereich CU sprechen die bisherigen Studiendaten und klinischen Erfahrungen für eine vergleichbar gute Wirksamkeit von Biosimilars wie das Original-Biologikum Infliximab ohne wesentliche Änderungen im Nebenwirkungsspektrum. Negative Daten, die gegen eine Anwendung von Biosimilars bei chronisch-entzündlichen Darmerkrankungen sprechen würden, wurden bisher weder in Studien publiziert, noch sind diese aus der klinischen Anwendung bekannt. Der mehr als 10-jährige Einsatz von Biosimilars in anderen Indikationsbereichen unterstreicht die Unbedenklichkeit zumindest bei der Neueinstellung auf in Studien geprüfte Biosimilars.

Die bisherigen Daten zur Umstellung vom Original auf ein Infliximab-Biosimilar (z. B. aus der NorSwitch-Studie [419]) ergaben 
bisher ebenfalls keine relevanten Hinweise auf einen Wirksamkeitsverlust, eine vermehrte Rate an Nebenwirkungen oder Immunogenitätsprobleme. Da die Datenlage dazu jedoch noch deutlich weniger robust ist im Vergleich zur Neueinstellung, sollten weitere Studiendaten gesammelt werden, um auch diesbezüglich eventuell vorhandene Bedenken auszuräumen. Gastroenterologen sollten sich intensiver mit dem Biosimilar-Konzept bei chronisch-entzündlichen Darmerkrankungen vertraut machen und unbegründete Ängste in Bezug auf die Sicherheit und Effektivität dieser Substanzen abbauen. Unabhängig von der bisher bestehenden relativen Unbedenklichkeit der Anwendung des Biosimilars Infliximab in der Erstanwendung und vermutlich auch bei einem Wechsel sind höchste Anforderungen an die Qualitätskontrollen im Herstellungsprozess von Biosimilars zu stellen. Eine größtmögliche Transparenz in Bezug auf den Herstellungsprozess wie auch auf die Verordnung ist zu fordern. Grundsätzlich sollte in Bezug auf den Einsatz von Biologika bzw. Biosimilars gefordert werden, dass der behandelnde Arzt die von ihm verordneten Produkte eindeutig zuordnen kann und zumindest in Bezug auf einen Wechsel zwischen Originalprodukt und Biosimilar einer laufenden Therapie weiterhin die Entscheidungshoheit behält. Ein wiederholter Wechsel von Biosimilar mit Original bzw. zwischen verschiedenen Biosimilars (sog. „MultiSwitch") ist abzulehnen, da dazu keine Daten vorliegen.

\section{Remissionserhaltung bei kompliziertem Verlauf einer Colitis ulcerosa}

\section{EMPFEHLUNG 3.33}

Nach Erreichen einer Remission sollte eine remissionserhaltende Therapie mit Thiopurinen bei Patienten mit milder bis moderater Erkrankungsaktivität eingesetzt werden, wenn frühe oder gehäufte Schübe unter einer optimal dosierten Therapie mit 5-Aminosalizylaten auftreten oder eine Unverträglichkeit gegen 5-Aminosalizylate besteht (Evidenzgrad 1), wenn ein steroidabhängiger Erkrankungsverlauf besteht (Evidenzgrad 2) oder wenn ein Ansprechen auf eine remissionsinduzierende Therapie mit Ciclosporin oder Tacrolimus besteht (Evidenzgrad 3)

Empfehlungsgrad B, starker Konsens

\section{Hintergrund}

Mehrere randomisiert-kontrollierte Studien haben die Wirksamkeit von Thiopurinen (Azathioprin und Mercaptopurin) in der Remissionserhaltung der Colitis ulcerosa untersucht [401, 420 - 425]. Eine Cochrane-Metaanalyse mit 7 dieser Studien und 302 Patienten kommt zu der Schlussfolgerung, dass die Effektivität von Thiopurinen insgesamt mäßig ist und die Evidenz für den Einsatz von Thiopurinen bei Colitis ulcerosa deutlich schwächer ist als bei Morbus Crohn [233]. Die Studienqualität in den älteren Studien ist insgesamt nicht mehr vergleichbar mit den Ergebnissen heutiger Studien. Unklar ist aufgrund dieser Studien auch, ob eine Begleitmedikation mit 5-ASA eine Therapieoptimierung ermöglicht. Zu beachten ist eine hohe Rate von azathioprininduzierten Nebenwirkungen, insbesondere Auslösung einer akuten Pankreatitis, Hepatopathie und Knochenmarkssuppression. Eine zusätzliche Evidenz für die Anwendung von Thiopurinen bei Colitis ulcerosa ergibt sich aus re- trospektiven Studien [426-431]. Eine Studie aus Oxford mit 346 Patienten zeigte eine Remissionsrate von 58 \% mit Azathioprin, die auf $87 \%$ erhöht werden konnte, wenn die Patienten für mindestens 6 Monate mit Azathioprin behandelt wurden. Die Remissionsrate nach 5 Jahren lag bei $62 \%$ bei einer strengen Definition des Rezidivs und bei $81 \%$, wenn leichtere Schübe mit einem kurzen Steroidstoß behandelt werden konnten. Die mediane Zeit bis zum erneuten Schub nach Beendigung der Therapie mit Azathioprin lag bei 18 Monaten [427]. Eine neuere retrospektive Studie zeigt Rezidive bei etwa 1/3 der Patienten 3 Jahre nach Beendigung einer Thiopurin-Therapie, wobei insbesondere bei einer ausgedehnten Colitis ulcerosa biologische Hinweise für eine erhöhte entzündliche Aktivität zum Zeitpunkt der Therapiebeendigung oder nur eine kurze Therapie mit Thiopurinen bestanden [432].

Calcineurininhibitoren (Cyclosporin A, Tacrolimus) werden als Rescue-Therapie bei steroidrefraktären Verläufen einer Colitis ulcerosa eingesetzt. Aufgrund ihres Nebenwirkungsprofils sollten sie nach Möglichkeit innerhalb von 6 Monaten abgesetzt werden, sodass sie eine wichtige Rolle als Bridging-Therapie spielen, bis CED-Therapeutika mit verzögertem Wirkeintritt wirksam sein können. Thiopurine sollten in dieser Situation bereits überlappend mit Calcineurininhibitoren gegeben werden, sobald ein eindeutiges Ansprechen auf die Calcineurininhibitoren zu erkennen ist. Parallel sollten Steroide ausgeschlichen werden. Durch den Einsatz von Thiopurinen zusammen mit Calcineurininhibitoren ist es möglich, die hohe Kolektomierate nach einer Monotherapie mit Calcineurininhibitoren zwischen 36 und 69\% nach 12 Monaten signifikant zu reduzieren [369, 370, 398, 399]. Retrospektive Fallserien konnten zeigen, dass durch Thiopurine das Risiko einer Kolektomie nach Anwendung von Calcineurininhibitoren reduziert werden kann [369, 370, 433]. Eine Beendigung der Calcineurininhibitorgabe sollte unter Berücksichtigung des verzögerten Wirkungseintritts von Thiopurinen nach 3-6 Monaten erfolgen.

Eine optimale Dosis für Azathioprin wurde bei der Indikation Colitis ulcerosa durch Studien nicht festgelegt. Als Dosierung für Azathioprin werden in Analogie zum Morbus Crohn üblicherweise 1,5 - 2,5 mg/kg Körpergewicht pro Tag bzw. die Hälfte der Dosis für 6-Mercaptopurin verwendet. Aufgrund der pharmakodynamischen Eigenschaft der Substanz (Wirkungseintritt erst nach 6-12 Wochen) ist eine längerfristige Therapieplanung erforderlich.

\section{EMPFEHLUNG 3.34}

Bei Patienten, die auf eine remissionsinduzierende Therapie mit TNF-Antikörpern ansprechen, ist eine remissionserhaltende Therapie mit TNF-Antikörpern mit oder ohne Thiopurinen sinnvoll.

Evidenzgrad 1, Empfehlungsgrad A, starker Konsens

Bei Patienten, die auf eine Induktionstherapie mit Vedolizumab ansprechen, ist eine Remissionserhaltung mit Vedolizumab sinnvoll.

Evidenzgrad 1, Empfehlungsgrad A, starker Konsens 
- Tab. 8 Übersicht Remissionsraten bei TNF-alpha-Antikörpern.

\begin{tabular}{|c|c|c|c|}
\hline TNF-alpha-Antikörper & Studie & Zeitpunkt & Remissionsraten \\
\hline \multirow[t]{2}{*}{ Infliximab } & ACT-1 [350] & Woche 54 & $\begin{array}{l}35 \% \text { IFX } 5 \text { mg } / \mathrm{kg} \\
34 \% \text { IFX } 10 \mathrm{mg} / \mathrm{kg} \\
17 \% \text { Plazebo }\end{array}$ \\
\hline & ACT-1 [350] & Woche 30 & $\begin{array}{l}26 \% \text { IFX } 5 \mathrm{mg} / \mathrm{kg} \\
36 \% \text { IFX } 10 \mathrm{mg} / \mathrm{kg} \\
11 \% \text { Plazebo }\end{array}$ \\
\hline \multirow[t]{3}{*}{ Adalimumab } & \multirow[t]{2}{*}{ ULTRA-2 [351] } & Woche 8 & 16,5\% Adalimumab 9,3\% Plazebo \\
\hline & & Woche 52 & $\begin{array}{l}\text { 17,3\% Adalimumab } \\
8,5 \% \text { Plazebo }\end{array}$ \\
\hline & $\begin{array}{l}\text { Gepoolte Daten ULTRA-1,-2,-3 } \\
\text { [436] }\end{array}$ & Woche 208 & $24,7 \%$ Adalimumab \\
\hline Golimumab & PURSUIT-M [353] & Woche 54 & $\begin{array}{l}27,8 \% \text { (klinische Remission) bzw. } 42,4 \% \text { (Mukosaheilung) } \\
\text { unter Golimumab } 100 \text { mg s. c. alle } 4 \text { Wochen; } \\
\text { Plazebo mit 15,6\% klin. Remission bzw. 26,6\% Mukosaheilung }\end{array}$ \\
\hline
\end{tabular}

\section{Hintergrund}

Remissionserhalt mit TNF-Antikörpern

In den ACT-Studien konnte gezeigt werden, dass Patienten unter Infliximab-Therapie zu den Zeitpunkten Woche 30 und Woche 54 (nur ACT-1-Studie) im Vergleich zu Plazebo signifikant häufiger in Remission blieben ( $\triangleright$ Tab. 8). Zu beachten ist, dass der Anteil der Patienten mit steroidfreier Remission, wenngleich statistisch signifikant, gering ausfiel. In ACT-1 lag die steroidfreie Remission in Woche 54 bei $24 \%$ (IFX $5 \mathrm{mg} / \mathrm{kg}$ ), $19 \%$ (IFX $10 \mathrm{mg} /$ $\mathrm{kg}$ ) bzw. $10 \%$ (Plazebo), in ACT-2 waren es in Woche $3018 \%$ (IFX $5 \mathrm{mg} / \mathrm{kg}$ ), $27 \%$ (IFX $10 \mathrm{mg} / \mathrm{kg}$ ) und $3 \%$ (Plazebo) [350].

In einer Studie wurde der Langzeitverlauf von 121 Patienten mit refraktärem Verlauf einer Colitis ulcerosa, die mit IFX behandelt wurden, berichtet [434]. 67\% der Patienten sprachen auf die Therapie mit IFX an, davon hatten $68 \%$ ein anhaltendes klinisches Ansprechen (medianer Beobachtungszeitraum 33,0 Monate; 17,0 - 49,8 Monate), insgesamt $17 \%$ wurden kolektomiert.

Für Adalimumab konnte ebenfalls eine Überlegenheit im Remissionserhalt gegenüber Plazebo gezeigt werden. So lagen die Remissionsraten in der ULTRA-2-Studie in Woche 8 bei 16,5\% (Adalimumab) bzw. 9,3\% (Plazebo; $p=0,019$ ) und in Woche 52 bei 17,3\% (Adalimumab) vs. 8,5\% (Plazebo, $p=0,004$ ) [351]. Da Infliximab zum Zeitpunkt der ULTRA-2-Studie bereits zugelassen war, fand sich ein relevanter Anteil (40\%) von Patienten, die zuvor mit IFX behandelt wurden. Für diese Patienten fand sich hinsichtlich einer Remission in Woche 8 kein Unterschied zu Plazebo, in Woche 52 befanden sich lediglich 10,2\% dieser Patienten in Remission (Plazebo $3 \%$; $=0,039$ ). In einer Übersichtsarbeit aus 2015 mit Einschluss einzelner Fallserien wurden bei IFX-vorbehandelten Patienten Remissionsraten von 0 - $50 \%$ berichtet [413, 435]. Durch eine langfristige Adalimumab-Therapie über 52 Wochen konnte zudem die Hospitalisierungsrate gesenkt werden [435]. Daten zum Einsatz jenseits von Woche 52 stammen aus der gepoolten Analyse von ULTRA-1, ULTRA-2 und ULTRA-3 [436]. Für 199 Patienten liegen Daten zur Therapie über vier Jahre vor, die Remissionsrate in Woche 208 lag dabei bei 24,7\%, ca. $60 \%$ der Patienten, die nach einem Jahr in Remission waren, blieben auch in Woche 156 noch in Remission.

In der PURSUIT-M-Studie wurde die Wirksamkeit von subkutanem Golimumab bei Patienten mit Colitis ulcerosa, die zuvor keine TNF-Antikörper-Therapie erhalten hatten, gezeigt [353]. Bei Patienten, die in Woche 6 auf Golimumab angesprochen hatten, war dies in Woche 54 noch bei $47 \%$ (50 mg Golimumab s. c. alle 4 Wochen) bzw. 49,7\% (100 mg Golimumab s. c. alle 4 Wochen) der Fall, im Vergleich zu 31,2\% unter Plazebo. Unter 100 mg Golimumab s. c. alle 4 Wochen wurde eine klinische Remission mit 27,8\% und Mukosaheilung mit 42,4\% in Woche 54 berichtet (Plazebo 15,6\% bzw. 26,6\%).

Remissionserhalt mit Vedolizumab

Der Nachweis der Wirksamkeit einer längerfristigen Therapie mit Vedolizumab konnte durch die GEMINI-I-Studie erbracht werden [404]. Unter 8-wöchentlicher Therapie mit 300 mg Vedolizumab wurde eine Remissionsrate von $41,8 \%$ in Woche 52 berichtet, unter 4-wöchentlicher Therapie lag die Rate bei 44,8\% (Plazebo 15,9\%). Allerdings konnte kein signifikanter Unterschied zwischen der 4- und der 8-wöchentlichen Applikation von Vedolizumab herausgearbeitet werden [437]. In einer Subgruppenanalyse der GEMINI-I-Studiendaten erwies sich Vedolizumab sowohl bei TNF-Antikörper-naiven als auch -exponierten Patienten als wirksam, wenngleich die Remissionsraten bei den TNF-Antikörper-Versagern geringer waren $(46,9 \%$ versus $36,1 \%$ in Woche 52) [438]. In einer kürzlich publizierten Follow-up-Studie zeigte sich auch drei Jahre nach erfolgreicher Induktionstherapie noch bei $50 \%$ der Patienten, die initial auf Vedolizumab angesprochen hatten, eine endoskopische Mukosaheilung [439]. Real-World-Daten belegen in einer deutschen Kohorte eine steroidfreie klinische Remission nach 54 Wochen bei $22 \%$ der initial auf Vedolizumab eingestellten Patienten [440]. Bei 56\% dieser Patienten wurde Vedolizumab bereits nach einem medianen Zeitraum von 18 Wochen gestoppt. In einer französischen Kohorte betrug die steroidfreie klinische Remission bei Patienten unter Vedolizumab nach einem Jahr 40,5\%. Der überwiegende Teil der Patienten, die sich zu 
Woche 22 in Remission befanden, zeigte auch in Woche 54 noch eine klinische Remission [441]. In einer Metaanalyse konnte die remissionserhaltende Wirkung von Vedolizumab in der Prävention eines Rezidivs bestätigt werden [442].

\section{Kombinationstherapien im Remissionserhalt}

In der UC-SUCCESS-Studie wurde die Wirksamkeit der Kombinationstherapie aus Azathioprin (2,5 mg/kg Tagesdosis) und Infliximab $(5 \mathrm{mg} / \mathrm{kg}$ Woche $0,2,6,14)$ hinsichtlich Remission in Woche 16 untersucht [403]. Die Kombinationstherapie erwies sich mit einer steroidfreien Remissionsrate von 39,7\% in Woche 16 der jeweiligen Monotherapie der Einzelsubstanzen überlegen (IFX-Monotherapie 22,1\%; Azathioprin-Monotherapie $23,7 \%$ ). Eine Mukosaheilung trat bei 62,8\% unter Kombinationstherapie auf, unter IFX-Monotherapie bei 54,6\% im Vergleich zu 36,8 \% bei Azathioprin-Monotherapie. Zu beachten ist jedoch, dass die Remissionsrate nur in Woche 16 und nicht über einen längeren Zeitraum untersucht wurde. Zudem waren die eingeschlossenen Patienten naiv für eine Therapie mit Thiopurinen und TNF-Antikörpern.

Belastbare Daten für eine Kombinationstherapie aus Azathioprin mit Adalimumab oder Golimumab I liegen für die Therapie der Colitis ulcerosa nicht vor. In einer retrospektiven Analyse von 23 Patienten, die unter Adalimumab-Therapie einen Wirkverlust erlitten und infolgedessen einen Immunmodulator erhalten hatten (Thiopurine $n=14$; MTX $n=9$ ), kam es bei 11 Patienten (48\%) zu einer Rückbildung der Anti-Adalimumab-AK, einem Wiederanstieg des Adalimumab-Talspiegels und einem klinischen Ansprechen [443]. Allerdings befanden sich unter diesen 23 Patienten nur zwei mit Colitis ulcerosa, sodass die Ergebnisse keine allgemeine Empfehlung für eine Kombinationstherapie mit Adalimumab erlauben. Prospektive Daten zur Behandlung des Morbus Crohn zeigen in der Kombinationstherapie keinen signifikant besseren Verlauf verglichen mit der Adalimumab-Monotherapie, wenngleich bei dem sekundären Endpunkt Mukosaheilung ein Vorteil der Kombinationstherapie gezeigt werden konnte [444].

Studien, die die einzelnen Substanzen im direkten Vergleich hinsichtlich ihrer Wirksamkeit im Remissionserhalt untersuchten, fehlen. Eine Empfehlung, welche Substanz zu bevorzugen ist, kann daher nicht abgegeben werden. Üblicherweise wird jedoch die Erhaltungstherapie mit der Substanz durchgeführt, mit der eine Remissionsinduktion erreicht werden konnte.

\section{EMPFEHLUNG 3.35}

Methotrexat, Ciclosporin und Tacrolimus sollten zur Remissionserhaltung der Colitis ulcerosa nur in Ausnahmesituationen eingesetzt werden.

Evidenzgrad 4, Empfehlungsgrad B, starker Konsens

\section{Hintergrund}

Bisher liegen keine ausreichenden Studiendaten vor, die einen Einsatz von Methotrexat oder Tacrolimus zur Remissionserhaltung nahelegen würden. Eine randomisierte, plazebokontrollierte Studie mit Methotrexat mit 67 Patienten zeigte, dass eine orale
Therapie mit 12,5 mg Methotrexat pro Woche keine Remissionserhaltung über einen Zeitraum von 9 Monaten ermöglicht [445].

Verschiedene retrospektive Studien haben sich mit der Wirksamkeit von Methotrexat in der Remissionserhaltung der Colitis ulcerosa beschäftigt. Die meisten Patienten wiesen ein Therapieversagen oder eine Unverträglichkeit auf Azathioprin auf. Die Ansprechraten bzw. die Remissionsraten lagen in diesen Studien zwischen 30 und $80 \%$ [424, 446]. Insgesamt ist die Datenlage zur Remissionserhaltung bei Colitis ulcerosa sehr heterogen. Eine systematische Cochrane-Metaanalyse kam zu dem Ergebnis, dass Methotrexat für die Erhaltungstherapie der Colitis ulcerosa zum gegenwärtigen Zeitpunkt nicht empfohlen werden kann [447].

\section{EMPFEHLUNG 3.36}

Aufgrund fehlender Evidenz kann keine Empfehlung zur Dauer einer Remissionserhaltung mit Thiopurinen, TNF-Antikörpern und Vedolizumab gegeben werden. Eine langfristige Fortsetzung der Remissionserhaltung wird aber häufig notwendig sein.

Expertenkonsens, starker Konsens

\section{Hintergrund}

Zu keiner der genannten Substanzen liegen ausreichend Daten vor, weder um die optimale Therapiedauer noch um die optimalen Voraussetzungen, die Therapie zu beenden, abzuschätzen.

In einer retrospektiven Studie wurde die Wirksamkeit von Azathioprin bei 346 Patienten mit Colitis ulcerosa untersucht. Die Remissionsraten nach einem, zwei und fünf Jahren lagen bei $95 \%, 69 \%$ und $55 \%$ [427]. Als einschränkend muss der retrospektive Charakter beachtet werden, zudem waren Remission und Schub der CU lediglich über die Abwesenheit bzw. den Einsatz einer Steroidmedikation definiert.

Auch für den Einsatz von Biologika (TNF-Antikörper, Vedolizumab) ist die optimale Therapiedauer nicht bekannt. In den jeweiligen Zulassungsstudien, die für Infliximab [350, 351, 353] und Vedolizumab [404] eine Erhaltungstherapie über ca. ein Jahr vorsahen, konnte eine Überlegenheit im Remissionserhalt gegenüber Plazebo dokumentiert werden. Eine TNF-Antikörper-Therapie sollte daher über mindestens ein Jahr erfolgen. Inwieweit Patienten, die sich nach einem Jahr in Remission befinden, von einer Fortführung dieser Therapie profitieren und ob die Remission rein klinisch oder endoskopisch definiert sein sollte, ist derzeit nicht abschließend geklärt.

Nach Beendigung einer TNF-Antikörper-Therapie wird ein relevanter Anteil der Patienten binnen 12 Monaten nach Therapieende einen erneuten Schub erleiden. In einer Metaanalyse aus 2016 betrug die Rezidivrate 12 Monate nach Beendigung einer Therapie mit Infliximab oder Adalimumab bei Patienten mit Colitis ulcerosa $28 \%$, das Wiederansprechen auf die zuvor beendete Therapie betrug ca. 80 \% [80]. Eine weitere Metaanalyse, die sowohl Patienten mit Morbus Crohn als auch mit Colitis ulcerosa einschloss zeigte eine Relapserate von etwa $50 \% 2$ Jahre nach Beendigung einer TNF-Antikörper-Therapie [448]. 


\section{EMPFEHLUNG 3.37}

Der apathogene Escherichia-coli-Stamm Nissle 1917 kann in begründeten Fällen als Alternative zu 5-Aminosalizylaten eingesetzt werden.

Evidenzgrad 2, Empfehlungsgrad 0, starker Konsens

\section{Hintergrund}

In einer Metaanalyse aus 2015 wurden insgesamt sechs Studien zum Einsatz von E. coli Nissle (EcN) 1917 bei Colitis ulcerosa getestet. Dabei erwies sich EcN als gleich wirksam im Remissionserhalt im Vergleich zu Mesalazin [268]. Als einschränkend muss jedoch beachtet werden, dass in einigen der eingeschlossenen Studien auch nur gegen Plazebo getestet wurde, somit EcN nicht wirksamer war als Plazebo. Zum anderen lag in allen eingeschlossenen Studien die Mesalazindosis mit 500-800 mg Tagesdosis unter der als wirksam angesehenen Dosis (siehe Empfehlung 3.13). Die Studien wurden von einem großen Teil der Konsensusteilnehmer als nicht ausreichend valide angesehen.

\section{EMPFEHLUNG 3.38}

Ein therapeutisches Drug Monitoring kann unter einer Therapie mit Thiopurinen, TNF-Antikörpern und Vedolizumab durchgeführt werden, wenn dadurch eine klinische Entscheidung unterstützt wird.

Expertenkonsens, Empfehlung offen, starker Konsens Unter einer Therapie mit Calcineurininhibitoren sollen regelmäßig Spiegelbestimmungen durchgeführt werden. Expertenkonsens, starke Empfehlung, starker Konsens

\section{Hintergrund}

Trotz steigender Zahl verfügbarer medikamentöser Therapien bei Colitis ulcerosa und der Möglichkeit der Kombinationstherapie für gewisse Präparate ist die Zahl der effektiven Therapien weiterhin limitiert. Die Ansprech- und Remissionsraten unter einer Zweit- und Drittlinientherapie mit TNF-Antikörpern sind zudem sehr gering, sodass die verfügbaren Therapien in einer optimalen Dosierung und in optimalen Dosierungsintervallen angewendet werden sollten.

\section{Drug Monitoring bei TNF-alpha-Antikörpern}

Die Serumtalspiegel von Infliximab, Adalimumab und Golimumab korrelieren mit der klinischen Wirksamkeit, und hohe Talspiegel werden häufiger bei Patienten mit klinischer und endoskopischer Remission in Form einer Mukosaheilung gefunden [352, 449 - 453]. In einer kürzlichen Metaanalyse mit 3483 Patienten unter Einbeziehung von 22 Studien in den Indikationsbereichen Morbus Crohn und Colitis ulcerosa korrelierten Infliximab-Talspiegel $>2 \mu \mathrm{g} / \mathrm{ml}$ mit einem guten klinischen Ansprechen und niedrigen CRP-Spiegeln [454]. Als untere Grenzwerte für Talspiegel, die mit einer hohen Wahrscheinlichkeit mit klinischer Remission einhergehen, werden für Adalimumab Talspiegel von > $6 \mu \mathrm{g} / \mathrm{l}$ [449, 455] und für Golimumab Talspiegel von $>2,5 \mu \mathrm{g} / \mathrm{ml}$ in Woche 6 bzw. in der Erhaltungstherapie $>1,4 \mu \mathrm{g} / \mathrm{ml}$ berichtet [456]. Im
Fall von nachweisbaren, gegen Biologika gerichteten Antikörpern und gleichzeitig niedrigem Talspiegel führte eine Therapiefortführung bei CED-Patienten nur in $17 \%$ zum Erfolg, hingegen erbrachte ein Wechsel auf einen TNF-Antikörper ein Ansprechen von ca. $92 \%$ [457]. Dabei handelte es sich jedoch um eine retrospektive Auswertung. Weiterhin muss berücksichtigt werden, dass bei einem relevanten Anteil von Patienten, die nach Versagen einer IFX-Therapie auf Adalimumab umgestellt werden, Anti-Adalimumab-Antikörper auftreten werden, die mit einem Wirkverlust einhergehen [458]. Sollte trotz niedrigen Talspiegels und nachweisbarer Antikörper gegen einen TNF-Antikörper die Dosis erhöht oder das Applikationsintervall verkürzt werden, muss mit einer erhöhten Rate an allergischen Reaktionen gerechnet werden; in einer Metaanalyse aus 2014 erhöhten Anti-IFX-Antikörper das Risiko für eine allergische Infusionsreaktion um das 2,4-, schwere allergische Reaktionen sogar um das 5,8-Fache [459]. Liegen ein hoher Talspiegel und gleichzeitig Antikörper gegen TNF-Antikörper vor, sollte beachtet werden, dass derartige Antikörper teilweise transient vorkommen [460] und bei Fortführung der Therapie bei zwei von drei Patienten im Verlauf verschwinden [461]. In einer kürzlich durchgeführten Studie mit 247 Patienten konnte gezeigt werden, dass ein therapeutisches Drug Monitoring durch Messung von Talspiegeln und korrespondierenden Autoantikörpern bei mehr als $70 \%$ der CED-Patienten in therapeutischen Konsequenzen resultierte [462]. Nichtsdestotrotz kann das therapeutische Drug Monitoring nicht alle klinischen Fragen beantworten und zum gegenwärtigen Zeitpunkt aufgrund zahlreicher Limitationen allenfalls als eine von vielen Methoden bei der Therapiesteuerung der Colitis ulcerosa empfohlen werden.

\section{Drug Monitoring Vedolizumab}

Im Rahmen der Zulassungsstudie GEMINI-I wurde berichtet, dass ein höherer Talspiegel in Woche 6 mit höheren Ansprechund Remissionsraten assoziiert war [404]. Die ersten klinischen Erfahrungen mit der Talspiegelbestimmung bei Vedolizumab sind derzeit noch sehr begrenzt, deuten aber darauf hin, dass ein Vedolizumab Drug Monitoring zur Therapieoptimierung nützlich sein kann. Eine eindeutige Empfehlung zur Anwendung von Vedolizumab Drug Monitoring kann derzeit nicht gegeben werden [463-465].

\section{Drug Monitoring Calcineurininhibitoren}

Die effektiven Talspiegel bei Calcineurininhibitoren sind interindividuell sehr unterschiedlich, sodass zur Therapiesteuerung regelmäßige Talspiegelkontrollen und Dosisanpassungen erforderlich sind. Die Wirksamkeit von Ciclosporin und Tacrolimus bei Colitis ulcerosa wurde bereits ausführlich erläutert (siehe Hintergrund zu den Empfehlungen 3.26-3.28). Zusammengefasst wird für Ciclosporin A derzeit eine initiale Dosis von $2 \mathrm{mg} / \mathrm{kg} \mathrm{KG}$ mit nachfolgender Dosisanpassung gemäß Talspiegel empfohlen. Der optimale Spiegel ist weiterhin nicht bekannt, angestrebt werden sollen Talspiegel von $250-400 \mathrm{ng} / \mathrm{ml}$. Für Tacrolimus wird initial mit 0,05 mg Tacrolimus/kg KG zweimal täglich oral behandelt; nachfolgend soll die Therapie nach Talspiegel gesteuert werden, wobei auch in diesem Fall der optimale Talspiegel nicht bekannt ist. Empfohlen werden Talspiegel von $4-8 \mathrm{ng} / \mathrm{ml}$ [466], $5-10 \mathrm{ng} / \mathrm{ml}$ [375] bzw. $10-15 \mathrm{ng} / \mathrm{ml}$ [374]. Evidenzbasierte Empfehlungen zur Häufigkeit der Spiegelmessungen bestehen nicht. In der initialen Behandlungsphase werden die Spiegelmes- 
sungen häufiger erforderlich sein, beispielsweise einmal wöchentlich. Im Fall stabiler Spiegel unter stabiler Dosis kann die Häufigkeit der Talspiegelmessungen reduziert werden. Limitierend wirkt sich oftmals die talspiegelabhängige Nebenwirkungsrate aus, und nicht alle Patienten werden auf Dauer einen hohen Talspiegel $(10-15 \mathrm{ng} / \mathrm{ml})$ tolerieren.

\section{Infektiologische Probleme}

Das Verständnis der Pathogenese der Colitis ulcerosa konnte in den letzten Dekaden deutlich erweitert werden. Klinische und experimentelle Befunde weisen einer gastrointestinalen Barrierestörung und einer Fehlregulation des Immunsystems kausale Bedeutungen zu. Es gibt jedoch keine Erkenntnisse, die belegen, dass Patienten mit der Erkrankung selbst einen Immundefekt mit daraus resultierender erhöhter Suszeptibilität für bakterielle oder virale Infektionen entwickeln. Vielmehr bedingt die Therapie mit immunsuppressiven Medikamenten partielle Defizite im angeborenen und erworbenen Immunsystem, die gehäuft zu typischen und opportunistischen Infektionen führen können. Bei schweren Krankheitsverläufen kann die daraus resultierende Mangelernährung eine medikamenteninduzierte Immundefizienz verstärken.

\section{STATEMENT 4.1}

Bei einer Kombinationstherapie aus mehreren immunsuppressiv wirkenden Medikamenten steigt das Risiko für Infektionen mit Hinzunahme jedes weiteren Medikaments deutlich an. Ältere Patienten, Patienten mit Komorbiditäten, Patienten mit stattgehabten schweren Infektionskrankheiten und Patienten mit Mangelernährung sind durch Infektionen besonders gefährdet.

Evidenzgrad 3, starker Konsens

\section{Hintergrund}

Jedes immunmodulierend wirkende Medikament birgt für Patienten mit Colitis ulcerosa ein erhöhtes Infektionsrisiko, das aber nicht individuell quantifizierbar ist [306]. Eine Fall-Kontroll-Studie mit insgesamt 300 Patienten mit CED zeigt, dass das Risiko, an einer opportunistischen Infektion zu erkranken, bei einer Monotherapie mit Steroiden, Azathioprin/6-Mercaptopurin oder Infliximab im Gegensatz zu Patienten ohne immunsuppressive Therapie jeweils um den Faktor 3 erhöht war. Wurden jedoch zwei oder drei der genannten Medikamente in Kombination angewendet, kam es zu einem drastischen Anstieg des Infektionsrisikos (OR 14,5) [11]. Die Therapie mit Anti-Integrin-Antikörpern bedingt wahrscheinlich kein erhöhtes Infektionsrisiko [467].

In der Abschätzung des Risikos für therapieinduzierte Infektionen wird häufig vergessen, dass systemische Steroide ein relevantes Infektionsrisiko beinhalten [468]. Daten aus der Rheumatologie zeigen, dass bei Patienten mit einer Tagesdosis von mehr als $10 \mathrm{mg}$ Prednison oder einer kumulativen Dosis von mehr als $700 \mathrm{mg}$ oder einer Therapiedauer von mehr als zwei Wochen das Infektionsrisiko erhöht ist $[469,470]$. Eine Untersuchung von ca.
500 an Tuberkulose neuerkrankten Patienten zeigt, dass das Risiko, unter einer laufenden Steroidtherapie an einer aktiven Tuberkulose zu erkranken, im Vergleich zu Patienten ohne Steroide auf das Fünffache erhöht ist. Dabei hatten Patienten mit einer maximalen Steroidtagesdosis von weniger als $15 \mathrm{mg} / \mathrm{Tag}$ ein etwa 3-fach, mit mehr als 15 mg/Tag ein nahezu 8-fach erhöhtes Risiko [471].

Für CED-Patienten, die älter als 50 Jahre sind, ist gegenüber $<25$-jährigen Patienten ein dreifach höheres Risiko beschrieben, an Infektionen zu erkranken [11, 472, 473]. Mögliche Gründe für ein erhöhtes Infektionsrisiko im Alter sind Veränderungen sowohl im angeborenen als auch im erworbenen Immunsystem [474]. Daten zu Patienten mit rheumatoider Arthritis zeigen, dass Komorbiditäten (z. B. Nierenfunktionsstörungen, Diabetes mellitus) und aus der Anamnese zu erfragende, durchgemachte schwere Infektionskrankheiten weitere Risikofaktoren für die Entwicklung von Infektionen darstellen [475]. Auch bei CED-Patienten ist ein Diabetes als ein manifester Risikofaktor für Infektionen beschrieben worden [476]. Insbesondere bei älteren Patienten muss deshalb das medikamentös induzierte Infektionsrisiko gegenüber dem möglicherweise geringeren operationsspezifischen Risiko einer Proktokolektomie abgewogen werden.

\section{EMPFEHLUNG 4.2}

Bei Erstdiagnose bzw. spätestens vor Beginn einer immunsuppressiven Therapie sollte bei allen Patienten ein Infektionsscreening auf Hepatitis B, Tuberkulose und EBV durchgeführt werden. Vor Einleitung einer Therapie mit Biologika ist das Tuberkulose-Screening zu aktualisieren.

Evidenzgrad 3, Empfehlungsgrad B, Konsens

\section{EMPFEHLUNG 4.3}

Vor Beginn einer immunsuppressiven oder immunmodulatorischen Therapie bei Colitis ulcerosa sollten eine gezielte Anamnese, eine Röntgenaufnahme der Lunge und ein Interferon-gamma-Release-Assay (IGRA) erfolgen, um eine aktive oder latente Tuberkulose-Infektion auszuschließen.

Evidenzgrad 3, Empfehlungsgrad B, starker Konsens

\section{Hintergrund}

Eine immunsuppressive Therapie, inklusive einer TNF-Antikörpertherapie, bzw. eine Chemotherapie kann bei HBV-Trägern das Risiko einer HBV-Reaktivierung signifikant erhöhen. Die Inzidenz einer HBV-Reaktivierung während bzw. nach einer Chemotherapie beträgt bei HBs-Ag-Trägern $15-50 \%$ [477, 478]. Obwohl die HBV-Reaktivierung bei HBsAg-negativen Patienten deutlich seltener auftritt, wurde auch bei 14 - $20 \%$ der anti-HBc- und anti-HBspositiven Patienten mit Lymphomen unter einer Chemotherapie eine Reaktivierung beschrieben [479]. Eine spanische Studie beschreibt bei 3 von 80 Patienten mit Morbus Crohn eine chronische HBV-Infektion. Zwei der Patienten entwickelten nach Beendigung der Infliximab-Therapie eine schwere Hepatitis, der dritte 
Patient verstarb aufgrund von Komplikationen einer Leberzirrhose [480]. Diese Beispiele zeigen, dass die Problematik der Reaktivierung nicht nur in den Langzeitfolgen (z. B. Zirrhose oder hepatozelluläres Karzinom) besteht, sondern auch das Risiko einer akuten Exazerbation der Hepatitis B mit fulminantem Verlauf erhöht ist [481]. Somit wird für alle Patienten, bei denen eine immunsuppressive Therapie eingeleitet werden soll, vor Beginn der Behandlung ein Screening auf HbsAg- und Anti-HBc-Antikörper empfohlen. Bei seronegativen Patienten wird eine Impfung empfohlen. Eine medikamentös-präventive Therapie mit Nukleos(t)idanaloga wirkt der HBV-Reaktivierung entgegen und ist daher bei HBsAgpositiven Patienten indiziert. Bei HBs-Ag-negativen und anti-HBcAntikörper-positiven Patienten wird eine engmaschige ALT- und HBV-DNA-Kontrolle empfohlen. Bezüglich der Dauer und Art der medikamentös-präventiven Therapie sowie des Managements von HBs-Ag-negativen und anti-HBc-Antikörper-positiven Patienten wird auf aktuelle Leitlinien verwiesen [482].

Vor Beginn einer immunsuppressiven Therapie - streng genommen gehört auch dazu eine Steroidstoßtherapie - sollte ein Tuberkulose-Screening durchgeführt werden. Besser ist es, wenn dieses schon mit der Diagnosestellung einer CU durchgeführt wurde. Grundsätzlich stehen für die immunodiagnostische Testung der Tuberkulinhauttest (THT) bzw. Interferon-gammaRelease-Assay (IGRA), ggf. auch in Kombination mit einer Röntgen-Thoraxuntersuchung, zur Verfügung. Alle zur Verfügung stehenden Testverfahren haben Schwächen; für den IGRA wird die Gefahr eines falsch negativen Testergebnisses gering eingestuft, kann jedoch, vor allem bei Patienten mit schwerer Lymphopenie und Immunsuppression, nicht ausgeschlossen werden [483]. Für den THT gilt, dass eine abgeschwächte Immunkompetenz falsch negative Ergebnis bedingen kann; bei geimpften Personen fällt dieser falsch positiv aus [484]. Neben dem IGRA können auch durch eine Röntgenaufnahme der Lunge Zeichen einer durchgemachten, aber unbehandelten Tuberkulose ohne Anhalt für Aktivität (kalzifizierte Knötchen, Spitzenfibrose, Pleuraschwielen) erfasst und als Indikation zur Chemoprävention betrachtet werden (siehe Empfehlung 4.19).

Das Tuberkuloserisiko ist beim Einsatz von TNF-Antikörpern im Vergleich zu anderen immunmodulierenden/-suppressiven Medikamenten wahrscheinlich am größten. Vor diesem Hintergrund wird empfohlen, die Tuberkulosediagnostik vor Beginn einer Therapie mit TNF-Antikörpern zu aktualisieren, nachdem eine zwischenzeitliche Tuberkuloseneuinfektion seit Erstdiagnose der CED und Tuberkulose-Screening nicht ausgeschlossen werden kann. Verschiedene Fachgesellschaften empfehlen bei Patienten mit kontinuierlicher Anti-TNF-Therapie eine jährliche Wiederholung des TB-Screenings. Hintergrund sind Konversionsraten von TB-Screening-negativen zu TB-Screening-positiven Patienten von bis zu $30 \%$ [485]. Aufgrund der niedrigen Prävalenz der Tuberkulose in Deutschland kann dieser Empfehlung nicht gefolgt werden. Allerdings sollte bei Personen mit einem erhöhten Expositionsrisiko (z. B. Flughafenpersonal, Personen, die in Lungenkliniken arbeiten, Fernreisende in TB-Endemiegebiete etc.), das Screening jährlich wiederholt werden.

Wenn auch keine epidemiologischen Daten zu einem erhöhten Tuberkuloserisiko bei einer Therapie mit Anti-Integrin-Inhibitoren oder JAK-Inhibitoren vorliegen (in allen Zulassungsstudien wurden
Patienten mit latenter Tuberkulose ausgeschlossen), sollte auch unter Berücksichtigung der Zulassung eine Tuberkulose vor Einleitung einer Therapie ausgeschlossen werden.

Die Infektion mit dem Epstein-Barr-Virus (EBV) erfolgt zumeist im Kindesalter und verläuft häufig asymptomatisch. Bei Jugendlichen oder Erwachsenen manifestiert sich die Primärinfektion in 30-60\% aller Fälle als infektiöse Mononukleose. Ab dem 40. Lebensjahr sind ca. $95 \%$ der Menschen mit EBV infiziert. Nach einer asymptomatischen oder symptomatischen Infektion persistiert das EBV in B-Zellen in der Zirkulation unter der Kontrolle von EBV-spezifischen zytotoxischen T-Zellen lebenslang [486]. Ist die Immunsurveillance der T-Zellen gestört, z. B. in einer Posttransplantationssituation, kommt es zu einer verstärkten Proliferation EBV-infizierter B-Zellen mit dem Risiko der Entwicklung eines Post-Transplantations-B-Zell-Lymphoms bzw. einer lymphoproliferativen Erkrankung (PTLD) [487]. Dabei ist eine primäre EBVInfektion in den ersten Jahren nach Transplantation mit einem deutlich erhöhten Risiko für eine PTLD verbunden [488, 489].

\section{EMPFEHLUNG 4.4}

Bei Erstdiagnose, spätestens aber vor Einleitung einer immunsuppressiven Therapie, sollte der Impfstatus überprüft und ggf. aktualisiert werden. Nicht-Lebendimpfungen unter immunsuppressiver Therapie gelten als sicher, während Lebendimpfungen kontraindiziert sind. Vor diesem Hintergrund sollten insbesondere Impfungen gegen Pneumokokken und gegen Hepatitis B sowie gegen Influenza und die pandemische Grippe in Analogie zu den Empfehlungen des RKI zu „Impfungen bei Immunsuppression“ durchgeführt werden. Evidenzgrad 2, Empfehlungsgrad B, starker Konsens

\section{Hintergrund}

Patienten mit CU haben ein erhöhtes Risiko, an einer impfpräventablen Infektionskrankheit zu erkranken und auch deswegen hospitalisiert zu werden [490]. Mehrere Versorgungsforschungsprojekte ergaben, dass viele CED-Patienten nur einen unzureichenden Impfschutz aufweisen. Der wichtigste Vorbehalt gegen die Durchführung einer Schutzimpfung ist eine oftmals unreflektierte Angst vor Nebenwirkungen. Ebenso wurde häufig eine Verschlimmerung der Grundkrankheit durch eine Impfung befürchtet; die allermeisten Patienten sind aber grundsätzlich bereit, empfohlene Schutzimpfungen durchführen zu lassen [491].

Bei allen Patienten mit CU sollte bei Diagnosestellung und nachfolgend in regelmäßigen (z. B. jährlichen) Abständen die Einhaltung der allgemeinen Impfempfehlungen des RKI überprüft werden. Zum Zeitpunkt der Erstdiagnose ist es oft noch möglich, fehlende Lebendimpfungen (v. a. Masern, Röteln, Varizellen) nachzuholen. Nach Beginn einer systemischen immunsuppressiven Therapie sind Lebendimpfungen formal kontraindiziert, wenngleich wohl dennoch sicher durchführbar [492].

Es sollte berücksichtigt werden, dass unter einer laufenden Immunsuppression, insbesondere unter einer Anti-TNF-Therapie, sowie einer kombinierten Immunsuppression die Ansprechraten 
einer Impfung reduziert sind und ein entsprechendes Monitoring erfolgen sollte [493].

Unabhängig vom Krankheitsschweregrad und der Therapie sollte jährlich gegen Influenza A/B geimpft werden. Alle Patienten mit (potenziell notwendiger) immunsuppressiver Therapie sollen als Indikationsimpfung gegen Pneumokokken geimpft werden. Die Erstimpfung sollte möglichst mit PCV13 und die Nachimpfung mit PPV23 erfolgen. Individuell sinnvoll können Impfungen gegen Meningokokken, Hämophilus influenzae und spezifische Reiseimpfungen sein.

\section{EMPFEHLUNG 4.5}

Patienten, die eine dreifach immunsuppressive Therapie erhalten, sollten eine PJP-Prophylaxe erhalten.

Evidenzgrad 4, Empfehlungsgrad B, Konsens

\section{Hintergrund}

Das Auftreten einer PJP wurde während der Therapie mit Steroiden, Thioguaninen, MTX, Calcineurininhibitoren oder Biologika beobachtet, wobei die Calcineurininhibitor-Therapie und Kombinationstherapien das höchste Risiko zu tragen scheinen [494]. Die vorliegenden retrospektiven Studien zeigen eine relativ geringe absolute Inzidenz: In der Olmsted-County-Kohorte zeigten sich trotz unregelmäßiger Prophylaxe bei 937 Patienten (6066 Patientenjahre Follow-up) lediglich 3 Fälle einer PJP [495]. In einer Analyse von Kostenträgerdaten zeigte sich eine Risikoerhöhung von 3 auf 10,6/100 000 Personenjahre bei CED im Vergleich zur Allgemeinbevölkerung, aber ein weiterer Anstieg auf 32/100 000 Personenjahre für CED-Patienten unter Immunsuppression [496]. Systematisch erhobene Daten zum PJP-Risiko unter definierten immunsuppressiven Regimen bei CED liegen nicht vor, sodass der Empfehlung auch Erfahrungen aus anderen Grunderkrankungen zugrunde liegen (siehe dazu auch: [330]).

Die TMP/SMX-Prophylaxe ist hoch effektiv zur PJP-Prävention bei Kindern und Erwachsenen mit hämatologischen Erkrankungen, Knochenmarkstransplantation und Organtransplantation [497]. In der ECCO-Leitlinie von 2014 „Opportunistische Infektionen“ wird eine PJP-Prophylaxe unter Dreifachimmunsuppression, die einen CNI oder TNF-Antikörper beinhaltet, empfohlen, bei Zweifachimmunsuppression mit einem CNI soll eine Prophylaxe erwogen werden [330]. Eine CD4-Zellzahl <200/ $\mu$ stellt bei zahlreichen HIV-unabhängigen Erkrankungen einen wichtigen Risikofaktor für die PJP dar [498]; für eine dezidierte Empfehlung zum CD4-Monitoring bei CED ist die Datenlage jedoch unzureichend. Als prophylaktisches Regime wird dreimal pro Woche die Einnahme von Sulfamethoxazol/Trimethoprim (800/160 mg) empfohlen, die eine gute Verträglichkeit zeigt $[497,499]$. Bei einer Sulfonamidallergie wird die Hinzuziehung eines Infektiologen angeraten.

\section{EMPFEHLUNG 4.6}

Bei bekannter Colitis ulcerosa sollte bei schwerem akutem Schub, atypischer Symptomatik, therapierefraktärem Verlauf und vor Intensivierung einer immunsuppressiven Therapie eine mikrobiologische Diagnostik inklusive Untersuchungen auf Clostridium difficile erfolgen.

Evidenzgrad 3, Empfehlungsgrad B, starker Konsens

\section{Hintergrund}

Eine routinemäßige Diagnostik auf $C$. difficile bei leichten Schüben wird nicht empfohlen, da in dieser Situation C.-difficile-Infektionen (CDI) selten sind [42, 500]. Bei hospitalisierten Patienten und bei Patienten nach Antibiotikatherapie ist die Infektionsrate durch C. difficile jedoch höher. CED-Patienten mit einer CDI weisen, im Gegensatz zu CED-Patienten ohne C.-difficile-Erkrankung, eine längere Krankenhausverweildauer und eine vierfach höhere Mortalität auf. Daher ist bei ihnen ein Screening auf C. difficile zu empfehlen [52, 337, 501].

Glukokortikoide (OR 2,5), Immunomodulatoren (OR 1,6) und TNF-Antikörper (OR 2,7) sind Risikofaktoren für eine schwere CDI [502 - 504]. Erfahrungen bei CED-Patienten sowie aus dem Bereich der Transplantationsmedizin zeigen, dass eine immunsuppressive Therapie mit einer höheren Inzidenz (OR 2,5 für AZA/6MP oder MTX) und höherem Schweregrad der Erkrankung einhergeht $[52,505]$. Eine Steroidtherapie bedingt im Vergleich zu AZA/ 6-MP und MTX bei 10662 CED-Patienten eine Verdreifachung des Risikos für eine CDI (RR 3,4; $95 \%-K I$ 1,9-6,1) [503]. Weitere Risikofaktoren sind eine (stattgehabte) Antibiotikatherapie oder die Ernährung über eine nasogastrale Sonde/PEG [506]. CED-Patienten mit C.-difficile-Erkrankung weisen bei einer Protonenpumpeninhibitor-Therapie ein knapp vierfach erhöhtes Rezidivrisiko für eine C.-difficile-Colitis auf [339].

\section{EMPFEHLUNG 4.7}

Die Diagnostik auf eine Clostridium-difficile-Infektion sollte zeitnah durch ein sensitives Verfahren erfolgen.

Evidenzgrad 3, Empfehlungsgrad B, starker Konsens

\section{Hintergrund}

Der C.-difficile-Nachweis soll zeitnah durch eine sensitive Diagnostik erfolgen [338]. Dafür reicht in der Regel eine breiig-flüssige Stuhlprobe; eine Diagnostik aus geformtem Stuhl ist nicht indiziert. In besonderen Situationen, z. B. bei Patienten mit einem lleus, können Rektalabstriche verwendet werden. Ein allgemein akzeptierter labordiagnostischer Algorithmus existiert nicht; darauf weist auch die aktuelle ESCMID-Leitlinie (European Society of Clinical Microbiology and Infectious Diseases) hin [507]. Grundsätzlich ist zwischen einem ein- und einem mehrstufigen Vorgehen zu unterscheiden. Aufgrund des notwendigen Zeitaufwands sind der direkte Antigennachweis im Stuhl (Glutamat-Dehydrogenase [GDH]), der direkte Nachweis der Toxine A/B (sogenannte Schnelltests) bzw. molekularbiologische Testverfahren zum Toxin- 
nachweis geeignet [338]. Der GDH-Nachweis ist aber unspezifisch und muss durch einen zweiten spezifischen Test bestätigt werden. Bei schweren, nach dem IfSG meldepflichtigen Infektionen, bei Patienten mit wiederholten Rezidiven und im Rahmen nosokomialer Ausbrüche sollte zusätzlich ein kultureller Nachweis angestrebt werden. Das erlaubt den Nachweis epidemiologisch besonders bedeutsamer Stämme (z. B. Ribotyp 027) und die Antibiotikaresistenztestung [508]. Hinzuweisen ist auch auf die Tatsache, dass ein negativer Stuhltest auf C.-difficile-Toxine nicht ausreicht, um eine pseudomembranöse Colitis auszuschließen.

Bei CU-Patienten und anderen Patienten mit einer Immunsuppression reichen wohl niedrigere Toxinmengen (die der Diagnostik entgehen) aus, um eine pseudomembranöse Colitis auszulösen. Wiederholte Stuhluntersuchungen zum Toxinnachweis oder die Durchführung einer C.-difficile-Kultur mit anschließendem Toxinnachweis werden deshalb empfohlen [509]. In diesen Fällen kann auch eine Sigmoidoskopie weitere Informationen bringen, insbesondere bei refraktären Patienten mit negativen Stuhlkulturen [508]. Auch bei ihnen schließt ein negativer Befund aber die Infektion nicht aus, zumal das endoskopisch typische Bild mit Ausbildung von Pseudomembranen häufig nicht zu beobachten ist.

\section{EMPFEHLUNG 4.8}

In Abhängigkeit von der Schwere des Krankheitsbilds werden für die spezifische Primärtherapie der CDI folgende Empfehlungen gegeben:

- Bei leichter bis moderater Krankheitsausprägung und in Abwesenheit von Risikofaktoren für einen schweren Verlauf kann die Therapie mit Metronidazol $3 \times 400 \mathrm{mg} / \mathrm{Tag}$ p. o. für mindestens 10 Tage erfolgen.

- Bei schwerer Krankheitsausprägung oder bei Vorliegen von Prädiktoren für einen schweren Verlauf sollte die Therapie mit Vancomycin $4 \times 125-250 \mathrm{mg} / \mathrm{Tag}$ p. o. für mindestens 10 Tage erfolgen.

- Beim Rezidiv und zusätzlichem Vorliegen von Risikofaktoren für Komplikationen (Immunsuppression, Komorbidität, Notwendigkeit einer zusätzlichen Antibiotikatherapie) kann der Einsatz von Fidaxomicin $2 \times 200$ mg/Tag p. o. erwogen werden.

- Bei rezidivierender oder therapierefraktärer Clostridiumdifficile-Infektion kann ein fäkaler Mikrobiomtransfer durchgeführt werden.

Expertenkonsens, Empfehlung offen, starker Konsens

\section{Hintergrund}

Wichtig in der Behandlung einer CDI sind Kontaktisolation, Kittel- und Handschuhpflege sowie Händereinigung mit Seife und sporiziden Desinfektionsmitteln [510]. Für die medikamentöse Therapie einer C.-difficile-Infektion gibt es unterschiedliche Empfehlungen, die jedoch nahezu alle eine antibiotische Therapie mit Metronidazol oder Vancomycin einschließen und sich nur in der Dosierung und der Dauer der Medikation sowie der Art des primär zu verwendenden Antibiotikums unterscheiden. Kontrol- lierte Studien zur Behandlung einer C.-difficile-Infektion bei CEDPatienten fehlen. Die oben formulierten Empfehlungen werden auch durch die deutsche SK2-Leitlinie Gastrointestinale Infektionen und Morbus Whipple gestützt [338].

Schweregradabhängig wird primär eine antibiotische Therapie mit Metronidazol oder oralem Vancomycin empfohlen. Sofern der klinische Verlauf es zulässt, sollte bei jedem Patienten mit einer CDI zunächst eine laufende antibiotische Therapie beendet werden. Für Patienten mit Zeichen einer leichten bis moderaten Erkrankung wird eine Therapie mit Metronidazol $400-500$ mg p. o. alle 8 Stunden über 10 Tage empfohlen. Sollte es nach 3 5 Tagen zu keiner Besserung kommen, muss von einem Versagen der Therapie ausgegangen werden und ein Wechsel z. B. auf Vancomycin p. o. erwogen werden. Für Patienten mit schwerer CDI (Fieber, Schüttelfrost, hämodynamische Instabilität, Peritonitis, lleus, Leukozytose $>15 \times 10^{9} / \mathrm{L}$, Linksverschiebung, Laktaterhöhung, toxisches Megakolon) wird eine initiale antibiotische Therapie mit Vancomycin $125 \mathrm{mg} /$ alle 6 Stunden per os für 10 Tage empfohlen. Eine höhere Vancomycindosierung ergibt keinen zusätzlichen Nutzen [511]. Bei Patienten, bei denen eine orale antibiotische Therapie nicht möglich ist, kann die Vancomycinapplikation (500 mg in $100 \mathrm{ml}$ Kochsalzlösung alle $4-12 \mathrm{~h}$ ) in das Kolon und/oder eine Vancomycingabe (500 mg alle 6 Stunden) über die Nasensonde erfolgen.

Eine gleichzeitig mit der CDI durchgeführte immunsuppressive Therapie sollte so weit wie möglich pausiert oder beendet werden, da immunsuppressiv behandelte CED-Patienten eine höhere Morbidität (u. a. Kolektomie, toxisches Megakolon, Darmperforation) und Mortalität aufwiesen als die Vergleichsgruppe ohne Immunsuppression [339]. Unklar bleibt aber dabei, ob die immunsuppressive Therapie per se einen schwereren Krankheitsverlauf mit potenziell mehr Komplikationen anzeigt.

Die klinische Beurteilung des Ansprechens auf die antibiotische Therapie ist bei CED-Patienten mit C.-difficile-Infektion, die sich nicht in Remission befinden, bei fortbestehender Diarrhö schwierig. Eine erneute mikrobiologische Stuhldiagnostik als Erfolgskontrolle einer antibiotischen Therapie ist nicht hilfreich, da sowohl C. difficile als auch Toxin A und B noch Wochen nach einer erfolgreichen Behandlung im Stuhl nachgewiesen werden können [512]. Wird jedoch das Rezidiv einer Infektion vermutet, sollte eine erneute Stuhldiagnostik durchgeführt werden. Resistenzen gegen Metronidazol wurden beschrieben und müssen, auch wenn sie selten auftreten, bei Therapieversagern beachtet werden [513].

Eine retrospektive Beobachtungsstudie verglich die Therapieerfolge von Metronidazol und Vancomycin bei 62 Patienten mit CU. Patienten mit leichter Infektion hatten weniger stationäre Wiederaufnahmen ( $0 \%$ versus $31 \%$ ) und kürzere Krankenhausaufenthalte (6 versus 14 Tage) nach Vancomycintherapie $(n=13)$ verglichen mit einer Metronidazoltherapie $(n=29)$. Auch Patienten mit schwerer Infektion hatten weniger stationäre Wiederaufnahmen ( $0 \%$ versus $70 \%$ ), aber tendenziell längere Krankenhausaufenthalte (19 versus 11 Tage) nach Vancomycintherapie $(n=9)$ verglichen mit einer Metronidazoltherapie $(n=10)$. Diese Studie ist die erste Untersuchung, die dafür spricht, Vancomycin bei Patienten mit CU primär einzusetzen [514].

Fidaxomicin zeigt eine im Vergleich zu Vancomycin geringere Rezidivrate bei insgesamt gleichwertigem primärem Ansprechen 
bei Patienten ohne CED $[515,516]$. Hauptnachteil ist der hohe Preis. Weiterhin ist Fidaxomicin zur Therapie der CDI bei CED bisher nicht zugelassen. Eine als Kurzmitteilung publizierte pharmakokinetische Untersuchung zeigt keine Unterschiede in der Resorption von Fidaxomicin bei CDI-Patienten mit und ohne CED [517]. Andere Medikamente zur Behandlung einer C.-difficile-Erkrankung, wie z. B. Rifaximin, Fusidinsäure oder Nitazoxanide, können zum jetzigen Zeitpunkt nicht empfohlen werden [518]. Ob eine zusätzliche Gabe von Probiotika bei Antibiotikagabe vor der Entwicklung einer CDI bei CU-Patienten schützt, wie es für Patienten ohne CED beschrieben wurde, ist unklar [519].

Die Prognose einer fulminanten CDI ist ernst. Bei schwerem Krankheitsbild sollte daher im Rahmen einer interdisziplinären Betreuung frühzeitig auch die Möglichkeit der chirurgischen Intervention beachtet werden.

Das Rezidiv einer C.-difficile-Erkrankung soll wie eine primäre Episode behandelt werden, weitere Rezidive direkt mit Vancomycin $125 \mathrm{mg}$ /alle 6 Stunden für 10 Tage [338]. Bislang ungeklärt ist das Vorgehen bei Auftreten von mehr als zwei Rezidiven. Bei diesen Patienten ist das Rezidivrisiko besonders hoch. Nach dem zweiten Rezidiv kommt es in 40-60\% der Fälle zu weiteren CDIEpisoden. Kasuistisch und in Fallserien wurden Patienten mit multiplen CDI-Rezidiven erfolgreich mit Vancomycin in verschiedenen Ausschleich- und/oder Pulsschemata behandelt [520]. Theoretische Überlegungen begründen, dass durch die abschließende alternierende Gabe von Vancomycin die im Darm verbliebenen Clostridiensporen an den „vancomycinfreien Tagen“ aussporen können, um dann als vegetative Formen abgetötet zu werden.

Die Erstbeschreibung einer erfolgreichen therapeutischen Übertragung vom Stuhl eines gesunden Donors auf einen Patienten mit einer CDI erfolgte bereits 1958 [521]. Zahlreiche Studien konnten die Effektivität dieser „Stuhltransplantation“ belegen, die inzwischen als fäkaler Mikrobiomtransfer (FMT) bezeichnet wird. Die erste randomisierte kontrollierte Studie zeigte bei Patienten mit multiplen Rezidiven eine signifikante Überlegenheit des FMT gegenüber einer konventionellen Rezidivtherapie mit Vancomycin [522]. In dieser Indikation wird der FMT auch in aktuellen europäischen Leitlinien ausdrücklich als alternative Therapie empfohlen. Eine retrospektive Multicenterstudie zeigte bei 31 Patienten mit CU und 35 Patienten mit Morbus Crohn eine gute Wirksamkeit bezüglich der C.-difficile-Infektion: $79 \%$ nach dem ersten FMT, $88 \%$ nach dem zweiten FMT und $90 \%$ nach einer 3. FMT [523]. Wahrscheinlich ist die Effektivität des FMT bei rezidivierender CDI bei CED-Patienten etwas niedriger als bei Patienten ohne CED. Auch ist in ca. 15-20\% der Fälle bei CED-Patienten mit einem akuten Schub nach FMT zu rechnen [524]; so können auch extraintestinale Manifestationen der CED auftreten [525].

Nach wie vor bestehen Sicherheitsbedenken, die auch durch ein (kostenintensives Screening des Donors (z. B. auf HIV, Hepatitis, Enteropathogene, Stuhlparasiten, multiresistente Erreger) nicht vollständig ausgeräumt werden können. Um weitere Daten zur klinischen Wirksamkeit und der langfristigen Sicherheit eines Mikrobiomtransfers zu erhalten, wurde ein nationales, internetbasiertes Register eingerichtet, in dem u. a. Patientencharakteristika, Details zur Durchführung des Mikrobiomtransfers sowie ein längerfristiges Follow-up der Patienten erfasst werden (Einzelheiten unter: www.kim4.uniklinikum-jena.de).

\section{EMPFEHLUNG 4.9}

EBV-seronegative erwachsene Patienten sollten möglichst nicht mit Thiopurinen behandelt werden.

Expertenkonsens, Empfehlung, Konsens

Die Therapieentscheidung bei Kindern stellt eine Sondersituation dar und erfordert eine Risikoabwägung

Expertenkonsens, Empfehlung offen, mehrheitliche Zustimmung

\section{Hintergrund}

Allgemein wird akzeptiert, dass das Risiko für die Entwicklung einer Lymphomerkrankung bei CED-Patienten, insbesondere bei denen, die mit Thiopurinen behandelt werden, erhöht ist. So weist die CESAME-Kohorte unter Auswertung von über 20000 Patienten eine Erhöhung des Lymphomrisikos unter laufender Thiopurin-Therapie um das Fünffache auf [526]. Das absolute Risiko ist jedoch niedrig und drückt sich in einer zusätzlichen Lymphomerkrankung auf 300 - 1400 Patientenjahre aus. Nichtsdestotrotz ist die Bedeutung der EBV-Infektion beträchtlich. In der CESAMEKohorte der mit Thiopurinen behandelten Patienten waren 12 der 15 Lymphome PTLD-ähnlich und regelhaft EBV-assoziiert. Die primäre EBV-Infektion stellt in dieser Situation eine besondere Herausforderung dar: Von 6 Patienten unter 50 Jahren entwickelten 2 Männer eine fatale infektiöse Mononukleose mit lymphoproliferativer Folgeerkrankung [526]. Über diese schwerwiegende Komplikation wurde bereits früher in Kasuistiken berichtet [527 - 529].

\section{EMPFEHLUNG 4.10}

Bei einer EBV-(Re-)Infektion sollte eine immunsuppressive/ immunmodulierende Therapie pausiert werden. Expertenkonsens, Empfehlung, Konsens

\section{EMPFEHLUNG 4.11}

Bei einer EBV-assoziierten lymphoproliferativen Erkrankung sollte die immunmodulatorische Therapie mit Thiopurinen beendet werden.

Expertenkonsens, Empfehlung, starker Konsens

\section{Hintergrund}

Bei einer primären EBV-Infektion unter Immunsuppression werden eine Unterbrechung der Immunsuppression und ggf. eine antivirale Therapie in Kooperation mit Infektionsspezialisten empfohlen. Finden sich atypische Infiltrate in der intestinalen Mukosa bei EBV-positiven Patienten, kann eine Reduktion der Immunsuppression zu einer Kontrolle der Virusreplikation und zum Verschwinden der Infiltrate beitragen [530]. Die haemophagozytische Lymphohistiozytose (HLH), auch als MakrophagenAktivierungssyndrom (MAS) bekannt, ist als eine schwere Komplikation einer akuten EBV-Infektion unter Thiopurinen, die auch als letale Komplikation verlaufen kann, beschrieben. Eine aktuelle 
Übersicht fasst 50 Patienten mit einer Mortalität von 30 \% zusammen [531]. Die HLH erfordert neben der Unterbrechung der Therapie ggf. den Einsatz antilymphoproliferativer Medikamente [532-534].

\section{EMPFEHLUNG 4.12}

Bei Patienten mit einem unzureichenden Ansprechen auf eine medikamentöse Therapie, insbesondere eine systemische Steroidtherapie, sollte eine geeignete Diagnostik einer CMVNeuinfektion oder Reaktivierung durchgeführt werden. Expertenkonsens, Empfehlung, starker Konsens

\section{Hintergrund}

Die CMV-Seroprävalenz der Bevölkerung liegt bei 70-100\% und steigt mit dem Alter an. Patienten mit CU weisen diesbezüglich keine Unterschiede gegenüber der Normalbevölkerung auf. Ein von der klinischen Situation unabhängiges Screening ist daher nicht sinnvoll. Mehrere Studien zeigten einen erhöhten CMVNachweis bei Patienten mit systemischer Steroidtherapie [326, $535,536]$. Im Einzelfall ist es oft schwer einzuschätzen, ob Steroide die CMV-Replikation erleichtern oder ob eine vorab bestehende erhöhte CMV-Replikation zum vermeintlichen klinischen Erfordernis einer Steroidtherapie und ggf. -refraktarität führt. Für andere Immunsuppressiva wie Thiopurine, IL-2-Hemmer oder TNF-Blocker besteht eine noch uneinheitlichere und/oder mangelhaftere Datenlage. Bei inadäquater klinischer Besserung der CU-Aktivität und/oder Zeichen einer systemischen CMV-Infektion (insbesondere Fieber und Leukopenie) sollte auch bei diesen Patienten eine CMV-Diagnostik erfolgen.

Aufgrund der bislang uneinheitlichen Datenlage sollte eine CMV-Diagnostik nur dann erfolgen, wenn bei CMV-Nachweis eine antivirale Therapie sinnvoll erscheint. Diese Entscheidung ist in erster Linie von der Ausprägung der klinischen Symptomatik bestimmt [331, 537].

\section{EMPFEHLUNG 4.13}

Zur Diagnostik sollte ein immunhistochemischer CMV-Nachweis aus endoskopisch gewonnenen Proben und/oder ein molekularbiologischer Nachweis aus Gewebeproben oder ein molekularbiologischer Nachweis aus Vollblut erfolgen Evidenzgrad 3, Empfehlungsgrad B, Konsens

\section{Hintergrund}

Es existieren vollblut-, serum- und gewebebasierte Möglichkeiten der CMV-Diagnostik. In den letzten Jahren traten Direktnachweismethoden in der entzündeten Mukosa in den Vordergrund (Gewebe-CMV-PCR, CMV-Immunhistochemie), während serologische Verfahren (Serum-CMV-IgM) oder der Nachweis im Vollblut (CMV-PCR, pp65) weniger beforscht wurden. Die Probenentnahme für den immunhistochemischen oder molekularbiologischen Direktnachweis sollte möglichst aus ulzeriertem Gewebe - optimalerweise dem Ulkusgrund oder -rand - erfolgen [538 - 541].

\section{EMPFEHLUNG 4.14}

Der alleinige CMV-Nachweis sollte keine Therapie begründen. Die Therapieindikation kann sich aus dem klinischen Kontext ergeben. Die Akuttherapie sollte für mindestens 14 Tage durchgeführt werden.

Evidenzgrad 2, Empfehlungsgrad B, Konsens

\section{Hintergrund}

Für kein diagnostisches Verfahren gibt es definierte Grenzwerte, ab denen von einer hinreichenden diagnostischen Genauigkeit und ggf. antiviralen Therapienotwendigkeit auszugehen ist. Ebenso ist es möglich, dass der Gewebe-CMV-Nachweis bei Patienten mit einer milden subklinischen CMV-Reaktivierung oder einer kontinuierlichen CMV-Replikation ohne Einfluss auf den CU-Verlauf ist. Daher erfordert ein CMV-Nachweis bei Patienten mit aktiver CU erst im klinischen Kontext und unter Abwägung weiterer individueller Faktoren (u. a. klinischer Schweregrad der CU-Aktivität, Dauer und Intensität der Steroidtherapie) eine antivirale Therapie [56, 57, 542, 543]. Es besteht bislang kein akzeptierter Grenzwert der Viruslast in der Gewebe-CMV-PCR, sodass v. a. bei niedrigen Titern eine falsch positive Analytik in Betracht gezogen werden muss.

Es gibt keine zugelassene medikamentöse Therapie der CMVColitis bzw. der CMV-Reaktivierung bei CU. Übliche Off-LabelAnwendungen sind $5 \mathrm{mg} / \mathrm{kg}$ Ganciclovir iv. alle 12 Stunden oder aber die primäre (oder nach erfolgreichem Primäransprechen auf Ganciclovir, sekundäre) orale Therapie mit Valganciclovir $900 \mathrm{mg}$ alle 12 Stunden für 2 (bis zu 3) Wochen. Bei unzureichendem Ansprechen kann das nebenwirkungsreichere Foscarnet (z. B. $3 \times$ tgl. $60 \mathrm{mg}$ i.v. über mindestens eine Stunde) für ebenfalls 2 3 Wochen Therapiedauer erwogen werden [544]. Inwieweit und wie lange eine Fortsetzung in „prophylaktischer“ Dosierung mit z. B. $450 \mathrm{mg}$ Valganciclovir tgl. z. B. bei begleitender immunsuppressiver Therapie sinnvoll ist, wurde bislang nicht evaluiert.

\section{EMPFEHLUNG 4.15}

Bei klinisch schwerwiegendem Verlauf einer CMV-Krankheit, insbesondere schwerer CMV-Colitis, Meningoenzephalitis, Pneumonitis oder Hepatitis, sollte die begleitende Immunsuppression zumindest bis zur virustatisch induzierten Restitutio pausiert werden. Bei alleiniger intestinaler Symptomatik eines CU-Schubs mit CMV-Nachweis kann die Immunsuppression fortgeführt oder modifiziert werden.

Evidenzgrad 2, Empfehlungsgrad B, starker Konsens

\section{Hintergrund}

Auch bei differenzierter klinischer Abwägung ist es häufig nicht möglich, die Kausalität eines akuten und ggf. steroidrefraktären CU-Schubs bei erfolgreichem CMV-Nachweis im entzündeten Gewebe allein dem CMV zuzuordnen. Daher muss eine individualisierte multimodale Therapie mit z. B. Thiopurinen oder TNFAntikörpern gleichzeitig oder nachfolgend zur antiviralen Thera- 
pie erfolgen [542]. Das Risiko einer Verschlechterung der CMVInfektion bzw. der Notwendigkeit einer Kolektomie unter einer parallel fortgeführten Immunsuppression scheint nach aktueller Datenlage eher gering zu sein [545]. Möglicherweise ist eine negativ werdende Plasma-CMV-PCR unter antiviraler Therapie bei unzureichender klinischer Verbesserung der CU ein geeignetes Signal für eine immunsuppressive Therapieintensivierung [544]. Aufgrund der erhöhten Wahrscheinlichkeit der Notwendigkeit einer späteren Kolektomie bei CU-Patienten mit klinisch relevanten CMV-Reaktivierungen sollte ggf. bereits zu diesem Zeitpunkt eine Operation erwogen werden [546].

Bei schweren intestinalen und extraintestinalen Manifestationen einer CMV-Infektion - die jeweils häufig mit Fieber einhergehen - sollte eine Einweisung in eine Klinik mit interdisziplinärer Kompetenz erfolgen. In Analogie zur Leitlinie der „Transplantation Society International CMV Consensus Group“ sehen wir die Reduktion oder Pausierung der immunsuppressiven Therapie in dieser Situation als sinnvoll an [547].

\section{EMPFEHLUNG 4.16}

Bei nachgewiesener CMV-Erkrankung sollte bei Wiedereinführung bzw. Fortführung oder Intensivierung der immunsuppressiven Therapie eine Rezidivprophylaxe durchgeführt werden. Diese Therapie schließt sich der Akuttherapie an und sollte für 4-8 Wochen durchgeführt werden.

Expertenkonsens, Empfehlungsgrad B, starker Konsens

\section{Hintergrund}

Es gibt keine prospektiv-randomisierte Studie zur Evaluierung des Nutzens einer antiviralen Rezidivprophylaxe nach erfolgreicher virustatischer Therapie einer klinisch relevanten CMV-Infektion bei CU und nachfolgendem Wiederbeginn oder Modifikation der immunsuppressiven Therapie. In Anlehnung an Empfehlungen aus der Transplantationsmedizin kann eine Rezidivprophylaxe mit 450 - 900 mg Valganciclovir tgl. erfolgen [547]. Möglicherweise ist eine negativ werdende Plasma-CMV-PCR unter antiviraler Therapie bei unzureichender klinischer Verbesserung der CU ein geeignetes Signal für eine immunsuppressive Therapieintensivierung [544]. Eine Therapieintensivierung mit einem TNF-Antikörper ist - verglichen mit Azathioprin - mit einem deutlich geringeren Anstieg der CMV-Viruslast verbunden. Aufgrund der erhöhten Wahrscheinlichkeit der Notwendigkeit einer späteren Kolektomie bei CU-Patienten mit klinisch relevanten CMV-Reaktivierungen sollte jedoch in dieser Situation eine Proktokolektomie erwogen werden [546].

\section{EMPFEHLUNG 4.17}

Bei aktiver VZV-Infektion sollte eine Immunsuppression nicht begonnen werden.

Expertenkonsens, Empfehlung, starker Konsens

\section{EMPFEHLUNG 4.18}

Bei florider VZV-Infektion während einer immunsuppressiven Therapie sollte unverzüglich eine Therapie erfolgen. Falls möglich, sollte die immunsuppressive Therapie unterbrochen werden.

Expertenkonsens, Empfehlung, starker Konsens

\section{Hintergrund}

Alle Patienten mit CU sollten bei Erstdiagnose bezüglich ihrer VZV-Anamnese befragt und ihr Impfausweis sollte bezüglich einer Varizellenimpfung überprüft werden. Die akute Windpockeninfektion hat unter Immunsuppression ein deutliches Komplikationsrisiko (z. B. Varizellenpneumonie) mit erhöhter Mortalität. Daher sollten Patienten ohne Windpockenanamnese und bei nicht vorhandenem VZV-Titer - falls zeitlich möglich - vor Beginn der immunsuppressiven Therapie geimpft werden. Die im Erwachsenenalter sehr viel häufigere VZV-Reaktivierung birgt die Gefahr neurologischer Spätkomplikationen (Neuralgie) [548].

Dauer und Applikation (oral oder intravenös) der Therapie mit antiviralen Substanzen (z. B. Aciclovir $5 \times 800 \mathrm{mg}$, Valaciclovir $3 \times 1000 \mathrm{mg}$, Famciclovir $3 \times 250 \mathrm{mg}$, Brivudin $1 \times 125 \mathrm{mg}$ ) richten sich nach dem Schweregrad der Erkrankung. In der Regel ist eine Therapie für 5 - 10 Tage oder bis zur Krustenbildung der Hautläsionen ausreichend [549]. Die Immunsuppression kann dann wieder aufgenommen werden, wenn keine neuen Effloreszenzen mehr auftreten bzw. alle Läsionen verkrustet sind. Für eine Fortsetzung der antiviralen Therapie in „prophylaktischer Dosis“ sprechen bislang keine Daten.

Eine passive Immunisierung mit VZV-Immunglobulin ist bei immunsupprimierten Patienten (z. B. eine Mutter mit Thiopurin/ TNF-Antikörpertherapie) mit hohem Infektions- und Komplikationsrisiko (kombinierte Immunsuppression, enger Kontakt zu Infizierten, höheres Lebensalter) innerhalb von 3 Tagen nach Exposition zu erwägen.

\section{EMPFEHLUNG 4.19}

Bei Nachweis einer latenten Tuberkulose (LTBI) (Interferongamma-Release-Assay (IGRA)) soll eine chemopräventive Therapie mit Isoniazid nach RKI-Empfehlungen durchgeführt werden.

Expertenkonsens, starke Empfehlung, starker Konsens

Eine immunsuppressive Therapie sollte frühestens 4 Wochen nach Start der Chemoprävention begonnen werden. Expertenkonsens, Empfehlung, starker Konsens

\section{Hintergrund}

Es wird eine Therapie mit $5 \mathrm{mg} / \mathrm{kg}$, aber maximal $300 \mathrm{mg}$ Isoniazid (INH) täglich für 9 Monate empfohlen. In seltenen Fällen kommt es unter dieser Therapie zu einer INH-Hepatitis $(0,15 \%)$. Bei Isoniazid-Unverträglichkeit kann alternativ eine Chemoprävention mit Rifampicin über 4 Monate erfolgen, wobei dieses Regime für Patienten unter TNF-alpha-Therapie nicht ausreichend 
evaluiert ist. Ist der Patient aus einem Herkunftsland mit bekannt erhöhter INH-Resistenz (z. B. Russland) immigriert, wird eine chemopräventive Therapie mit Rifampicin und Pyrazinamid über 3 Monate empfohlen.

\section{EMPFEHLUNG 4.20}

Bei Nachweis einer aktiven Tuberkulose ist eine Kombinationstherapie gemäß den RKI-Empfehlungen einzuleiten.

Expertenkonsens, starke Empfehlung

Eine immunsuppressive Therapie, insbesondere eine AntiTNF-Therapie, sollte - bei stets strenger Indikationsstellung idealerweise erst nach Beendigung der Tuberkulosetherapie durchgeführt werden.

Expertenkonsens, Empfehlung, starker Konsens

\section{Hintergrund}

Bezüglich der Behandlung einer aktiven Tuberkulose wird auf die Empfehlungen des Deutschen Zentralkomitees zur Bekämpfung der Tuberkulose des Robert Koch-Instituts verwiesen sowie auf eine aktuelle amerikanische Leitlinie [550, 551]. Ob unter laufender tuberkulostatischer Therapie eine TNF-Antikörper-Therapie eingeleitet werden kann, ist nicht bekannt. Eine TNF-Antikörper-Therapie sollte idealerweise erst nach Beendigung der Tuberkulosetherapie durchgeführt werden. Eine kritische Diskussion mit dem Patienten und eine strenge Indikationsstellung für die TNF-Antikörper-Therapie werden insbesondere in dieser Situation empfohlen.

\section{EMPFEHLUNG 4.21}

Eine Vakzinierung mit Lebendimpfstoffen (Rotavirus) soll bei Neugeborenen, deren Mütter in der Schwangerschaft mit TNF-Antikörper behandelt wurden, für mindestens 9 Monate nicht durchgeführt werden.

Expertenkonsens, starke Empfehlung, starker Konsens

\section{Hintergrund}

TNF-Antikörper (mit Ausnahme von Certolizumab) sind plazentagängig und erreichen somit therapeutische Wirkspiegel im Fetus. Der Wirkspiegel im Neugeborenen zum Zeitpunkt der Geburt hängt ab vom Zeitpunkt der letzten Applikation in der Schwangerschaft [552]. Die meisten der von der STIKO in den ersten Lebensmonaten empfohlenen Impfungen basieren auf Totimpfstoffen. Diese gelten als unbedenklich. Seit einigen Jahren wird jedoch die orale Lebendimpfung gegen Rotavirus in den ersten Lebenswochen empfohlen. Diese muss vermieden werden. Bereits in der Schwangerschaft sollten die Mütter darauf hingewiesen werden. Die Lebendimpfstoffe für Masern, Mumps, Röteln und Varizellen werden erst ab Lebensmonat 11 - 14 empfohlen. Dies stellt in aller Regel kein Problem dar, da bis dahin eine komplette Clearance der Medikamente im Neugeborenen erfolgt ist.

\section{Chirurgie}

\subsection{Operative Verfahren}

\section{EMPFEHLUNG 5.1.1}

Als Standardoperation sollte eine restaurative Proktokolektomie durchgeführt werden.

Evidenzgrad 3, Empfehlungsgrad B, starker Konsens

\section{Hintergrund}

Die restaurative Proktokolektomie hat sich in den letzten 35 Jahren als Standardoperation zur Behandlung der therapierefraktären Colitis ulcerosa oder bei maligner Entartung im Rahmen der Erkrankung etabliert. Die ileoanale Pouchoperation erzielt für den Patienten eine bestmögliche Lebensqualität mit durchschnittlich 5- 6 Stuhlgängen pro Tag und dem Erhalt der Kontinenz in über $90 \%$ der Patienten [553, 554].

\section{EMPFEHLUNG 5.1.2}

Die restaurative Proktokolektomie sollte in der Regel mit protektivem Ileostoma erfolgen; eine einzeitige Operation sollte nur in selektionierten Einzelfällen erfolgen.

Evidenzgrad 2, Empfehlungsgrad B, starker Konsens

\section{Hintergrund}

Die Anastomoseninsuffizienz nach ileopouchanaler Anastomose beträgt ca. $10 \%$. Sowohl die absolute Rate als auch die klinischen Auswirkungen eines derartigen Lecks werden potenziell durch ein protektives Stoma verringert. Es ist wahrscheinlich, dass ein Leck auch die spätere Pouchfunktion kompromittiert; die Datenlage dazu ist aber widersprüchlich. RCTs mit adäquater Fallzahl liegen nicht vor. Eine große aktuelle Registerstudie aus Dänemark über den Zeitraum von 33 Jahren zeigte allerdings eine signifikante Assoziation eines späteren Pouchversagens mit dem Verzicht auf ein protektives Stoma [555].

Um Selektionskriterien zu identifizieren, bei deren Vorliegen in ausgewählten Fällen doch auf ein Schutzstoma verzichtet werden kann (einzeitiges Vorgehen), werteten zwei „High-Volume“-Zentren ihre Pouch-Datenbanken gemeinsam aus. Knapp 15\% der 3733 Patienten erhielten kein protektives Stoma. Mit dem Stomaverzicht signifikant assoziiert waren eine Stapleranastomose, das Fehlen von Steroiden in der präoperativen Medikation, die FAP oder ein Karzinom als OP-Indikation, weibliches Geschlecht und Alter unter 26 Jahren. Die postoperative Morbidität einschließlich der Anastomoseninsuffizienzrate unterschied sich dann nicht zwischen den Patienten mit und ohne Stoma [556].

Zusammengefasst sollte bei Patienten, bei denen wegen therapierefraktärer Situation eine restaurative Proktokolektomie erfolgt, grundsätzlich nicht auf ein Stoma verzichtet werden, da die potenziellen Nachteile eines Stomas durch die Vorteile bei den in der Regel schwer kranken Patienten mehr als aufgewogen werden [557]. 


\section{EMPFEHLUNG 5.1.3}

Der J-Pouch sollte die Pouchkonstruktion der Wahl sein, da er am einfachsten anzulegen ist und im Langzeitverlauf eine vergleichbare Funktion aufweist wie andere Konstruktionen. Evidenzgrad 1, Empfehlungsgrad B, starker Konsens

\section{Hintergrund}

Die verfügbaren Studien zeigen keinen eindeutigen funktionellen Vorteil für das J-Design. In einer Metaanalyse von 18 nicht randomisierten Studien (NRS) mit insgesamt 1519 Patienten ergab sich in Bezug auf frühe postoperative Komplikationen kein Unterschied. Bezüglich Stuhlfrequenz erwiesen sich W- und S-Pouch dem J-Pouch als überlegen, wohingegen hinsichtlich Pouchentleerungsstörungen der S-Pouch am schlechtesten abschnitt [558]. In einer neueren randomisiert-kontrollierten Studie (W- versus J-Pouch) lag nach einem Jahr die mediane Stuhlfrequenz pro 24 Stunden beim J-Pouch signifikant höher als beim W-Pouch (7 vs. 5), nach 9 Jahren hatten sich die Unterschiede angeglichen. Alle anderen untersuchten Parameter, darunter Einlagengebrauch, Inkontinenz und Lebensqualität, waren vergleichbar [559]. Der K-Pouch wurde in einer kleinen randomisierten Studie aus Norwegen mit dem J-Pouch verglichen, wobei sich keine signifikanten funktionellen Unterschiede ergaben [560].

Auch wenn es in den vorliegenden Studien tendenziell Vorteile für die anderen Pouch-Konfigurationen, vor allem im Kurzzeitverlauf, zu geben scheint, bleibt der J-Pouch aufgrund seines einfachen Designs der Standardpouch. Zudem erscheint die klinisch sehr quälende, in einem relevanten Anteil der Patienten auftretende Pouchentleerungsproblematik nach S- und W-Pouch in den verfügbaren Studien nicht ausreichend berücksichtigt.

\section{EMPFEHLUNG 5.1.4}

Die freie oder gedeckte Perforation sollte als Notfallindikation operiert werden.

Evidenzgrad 2, Empfehlungsgrad B, starker Konsens

\section{Hintergrund}

Die freie oder gedeckte Perforation stellt die schwerste Komplikation der Colitis ulcerosa dar. Die Beurteilung der klinischen Symptomatik wird durch eine bestehende Immunsuppression und/oder Antibiotikatherapie häufig erschwert, sodass eine operative Therapie vor Eintreten septischer Komplikationen durchgeführt werden sollte. In der Hälfte der Fälle geht der Perforation kein Megakolon voraus. Trotz operativer Therapie beträgt die Mortalität derzeit bis zu $27 \%$ [561, 562]. Um die Mortalität dieser schwersten Komplikation zu senken, stellt die rechtzeitige Operation die entscheidende Maßnahme dar [319, 392, 563]. Bei einer Notfalloperation stellt die Kolektomie mit Blindverschluss des Rektums und endständiger Ileostomie den primären Standardeingriff dar (siehe auch 5.1.5. und 5.1.6.) [564, 565].

\section{EMPFEHLUNG 5.1.5}

Bei einer therapierefraktären Blutung sollte bei fortgesetzter Transfusionspflichtigkeit dringlich operiert werden. Evidenzgrad 2, Empfehlungsgrad B, starker Konsens

\section{Hintergrund}

Die Inzidenz der schweren Blutung bei Patienten mit CU beträgt bis zu 4,5\% [566]. Sie ist verantwortlich für bis zu $5 \%$ der Notfalleingriffe. Als Operationsindikation wird entweder eine massive initiale Blutung mit Kreislaufinstabilität und Katecholaminpflichtigkeit oder im Verlauf ein Transfusionsbedarf von mehr als 4 Erythrozytenkonzentraten pro 24 Stunden angesehen. Für Kinder ist eine Transfusionsbedürftigkeit von 45 - $60 \mathrm{ml}$ Erythrozytenkonzentrat/kg KG in 24 Stunden, bei anhaltender Blutung über 2 - 3 Tage $30 \mathrm{ml}$ EK/ kg KG als lebensbedrohlich anzusehen. Wenn möglich, sollten Kinder mit schwerem akutem Schub einer Colitis ulcerosa in ein Zentrum mit kindergastroenterologischer und chirurgischer Expertise verlegt werden. Besteht eine der oben aufgeführten Konstellationen, sollte dringlich (innerhalb von 24 Stunden) kolektomiert werden, in der Regel als subtotale Kolektomie mit Absetzung im oberen Rektum [392].

\section{EMPFEHLUNG 5.1.6}

Patienten mit einem medikamentös therapierefraktären fulminanten Schub sollten dringlich operiert werden.

Evidenzgrad 3, Empfehlungsgrad B, starker Konsens

\section{Hintergrund}

Die Diagnose des therapierefraktären fulminanten Schubs wird interdisziplinär gestellt und orientiert sich an den Kriterien der schweren Colitis nach Truelove and Witts, ist aber selbst nicht einheitlich definiert. Dies erschwert die Interpretation der Literatur. Radiologisch weist eine Kolondilatation von $6 \mathrm{~cm}$ auf ein toxisches Megakolon hin [323, 567]. Der therapierefraktäre fulminante Schub stellt mindestens eine relative Operationsindikation dar, wenn sich über einen Zeitraum von 72 Stunden durch konservative intensivmedizinische bzw. medikamentöse Behandlung mit hochdosierten Steroiden keine substanzielle Verbesserung der Erkrankungsintensität erzielen lässt. Eine sinnvolle Alternative zu einer weiteren Steigerung der medikamentösen Therapie mit Calcineurininhibitoren oder mit TNF-Antikörpern stellt die Operation dar. Es ist dabei zu berücksichtigen, dass eine weitere Steigerung der medikamentösen Therapie mittels Calcineurininhibitor oder TNF-Antikörper die Operation in maximal 42 - 65\% der Fälle um mindestens 1 Jahr aufschieben kann [399, 568]. In einer multivariaten Analyse konnte auch gezeigt werden, dass eine spätere Operation (8 versus 5 Tage) bei schwerer akuter Colitis zu signifikant mehr Majorkomplikationen führt [319].

Zusammengefasst kann bei der fulminanten Colitis eine intensivierte medikamentöse Therapie über einen Zeitraum von maximal 5 - 7 Tagen erfolgen, sollte sich der Patient darunter nicht verschlechtern. Eine Verschlechterung unter der Therapie erfor- 
dert eine dringliche Operation innerhalb von 24 Stunden, weil dadurch Mortalität und Morbidität verringert werden. Liegt ein toxisches Megakolon vor, ist das Zeitfenster einer konservativen Therapie deutlich geringer und sollte 48 bis maximal 72 Stunden nicht überschreiten. Patienten, die sich unter der Therapie verschlechtern oder nicht verbessern, sollten wiederum dringlich operiert werden [562].

Kinder mit einem fulminanten Schub einer Colitis ulcerosa sollen in ein Zentrum mit gastroenterologischer und chirurgischer Expertise verlegt werden. Das Krankheitsbild ist bei Kindern sehr selten, und die klinischen Symptome unterscheiden sich durchaus von denen bei Erwachsenen. Hilfreich kann die Berechnung des PUCAI (pediatric ulcerative colitis activity index) sein [569]. Dabei beinhaltet ein Wert höher 45 Punkte an Tag 3 oder ein Wert höher 70 an Tag 5 eine hohe Sensitivität und Spezifität für „NichtAnsprechen auf Steroide“ [570].

\section{EMPFEHLUNG 5.1.7}

Ein trotz Einsatzes von Immunsuppressiva und/oder Biologika therapierefraktärer Verlauf sollte operiert werden.

Evidenzgrad 2, Empfehlungsgrad B, starker Konsens

\section{Hintergrund}

Nach Versagen einer intensivierten konservativen Therapie (Immunsuppressiva und/oder Biologika) ist die chirurgische Entfernung des Kolons indiziert. Die Operation kann je nach Dauer und Intensität der Vormedikation und der klinischen Symptomatik drei- oder zweizeitig erfolgen. Bei therapierefraktärem Verlauf sollte frühzeitig eine interdisziplinäre Betreuung durch einen Gastroenterologen und Chirurgen erfolgen. Insgesamt ist die Definition eines therapierefraktären Verlaufs in der klinischen Realität variabel, was häufig dazu führt, dass Patienten erst zu spät ernsthaft die Option einer Operation als Alternative zu einer weiteren Intensivierung der medikamentösen Therapie empfohlen bekommen. Bei schwerem Verlauf führt eine protrahierte Therapie aber zu einer Erhöhung der Morbidität [571]. Daher sollte ein Festhalten an einer intensivierten konservativen Therapie zeitlich limitiert bleiben. Das wird auch durch eine Studie aus Münster unterstützt, die zeigte, dass über $50 \%$ der Patienten retrospektiv gerne früher operiert worden wären [572]. Eine jüngere Metaanalyse zur Sinnhaftigkeit von Drittlinientherapien bei schwerer chronischer Colitis zeigte, dass zwar kurzzeitige Besserungen möglich sind, in der Regel die Notwendigkeit zur Kolektomie jedoch nur verzögert wird und höhere Nebenwirkungsraten zu erwarten sind [573].

\section{EMPFEHLUNG 5.1.8}

Eine elektive Operation kann bei Patientenwunsch erfolgen. Dabei sind die Risiken der konservativen Behandlungsstrategien gegen die Risiken einer Operation abzuwägen. Evidenzgrad 4, Empfehlungsstärke 0, starker Konsens

\section{Hintergrund}

Die Operation stellt eine gute Alternative zu einer langjährigen medikamentösen Therapie dar. Im Langzeitverlauf lässt sich trotz aller möglichen Komplikationen nach restaurativer Proktokolektomie mit ileoanalem Pouch eine gute Lebensqualität für über $90 \%$ aller Patienten erreichen. Insbesondere Patienten, die ein dauerhaftes Krankheitsgefühl beklagen, eine schlechte Medikamentencompliance haben oder unter Karzinomangst leiden, profitieren von einer Operation [553, 554, 566].

In einer aktuellen Fall-Kontroll-Studie zwischen Patienten nach Pouchanlage versus Patienten unter Anti-TNF-Therapie ergab sich kein Lebensqualitätsunterschied (IBDQ), aber Pouchpatienten hatten höhere „QUALIs“, und die Kosten für das Gesundheitssystem waren insgesamt niedriger [574].

Grundvoraussetzung für eine elektive Operation bei Patientenwunsch ist ein intensives Beratungsgespräch mit einem in der Pouchchirurgie erfahrenen Viszeralchirurgen. Vor der Entscheidung für eine Operation sollen funktionelle Beschwerden ausgeschlossen werden.

\section{EMPFEHLUNG 5.1.9}

Kinder und Jugendliche mit aktiver Colitis und Wachstumsstörungen trotz adäquater Therapie sollten nach Konsultation eines Kindergastroenterologen und Ausschluss anderer Ursachen proktokolektomiert werden mit Anlage einer pouchanalen Anastomose.

Evidenzgrad 4, Empfehlungsgrad B, Konsens

\section{Hintergrund}

Bei Kindern und Jugendlichen mit Colitis ulcerosa sind Wachstumsstörungen sehr viel seltener als beim Morbus Crohn und dann Folge einer anhaltenden Entzündungsaktivität oder einer zu langen und zu hoch dosierten Steroidbehandlung, die unbedingt vermieden werden muss. Andere Ursachen einer Wachstumsstörung (z. B. Zöliakie, Wachstumshormonmangel, konstitutionelle Wachstumsverzögerung) müssen präoperativ ausgeschlossen werden. Die medikamentöse Behandlung und die Operationsindikation sollen durch einen Kindergastroenterologen mitbeurteilt werden. Ein Aufholwachstum nach einer Operation ist nur bei präpubertären Kindern oder Kindern in frühen Pubertätsstadien zu erwarten, sodass trotz der schlechten Evidenzlage eine starke Empfehlung gerechtfertigt erscheint [575].

\section{EMPFEHLUNG 5.1.10}

Bei erhöhtem perioperativem Risiko, unter Berücksichtigung der perioperativen Medikation, sollte die Proktokolektomie dreizeitig durchgeführt werden.

Evidenzgrad 3, Empfehlungsgrad B, starker Konsens

\section{Hintergrund}

Die Durchführung der Operation in drei Schritten beinhaltet 1 . die subtotale Kolektomie mit endständigem Ileostoma, 2. die 
Restproktomukosektomie mit ileoanaler Pouchanlage und doppelläufigem lleostoma und 3. die lleostomarückverlagerung. Das dreizeitige Operationsverfahren bei erhöhtem perioperativem Risiko geht insgesamt mit einer geringeren Rate an Komplikationen einher als das ein- bzw. zweizeitige Verfahren [576578]. Ein erhöhtes perioperatives Risiko besteht bei langfristiger Steroidtherapie, Therapie mit Biologika, Immunsuppression, Mangelernährung (siehe Empfehlungen 6.1.2 - 6.1.9) und bei etwaigen Begleiterkrankungen [391, 579]. Die Einnahme von mehr als $20 \mathrm{mg}$ Prednisolon über einen Zeitraum von mehr als 6 Wochen geht mit einer erhöhten Komplikationsrate der operativen Therapie einher. Nach Möglichkeit sollte die Steroiddosis präoperativ reduziert und gleichzeitig das Auftreten eines Steroidentzugssyndroms vermieden werden. Bei Kindern ist die entsprechende Dosis bei $15 \mathrm{mg} / \mathrm{m}^{2} \mathrm{KOF}$ (bzw. 0,5 mg/kg) anzusetzen. In einigen Studien weisen Patienten unter Therapie mit TNF-Antikörpern ein erhöhtes Risiko für postoperative Komplikationen auf. Zusätzlich ist die dreizeitige Operation bei Patienten unter Therapie mit TNF-Antikörpern häufiger als bei Patienten ohne Therapie mit Biologika, weswegen ein potenzieller negativer Effekt dieser Medikamente auf die perioperative Komplikationsrate durch Auswahl der Operation mit geringstem Risiko (=Kolektomie mit Ileostoma) unterschätzt werden könnte [580]. Da keine prospektiv randomisierte Studie vorliegt, ist nicht eindeutig geklärt, ob die erhöhte Komplikationsrate evtl. durch die schwerere Grunderkrankung der dann mit Biologika behandelten Patienten bedingt ist. In einigen Fall-Kontroll-Studien wurde eine erhöhte Zahl von operativen Komplikationen (z. B. Insuffizienz von Anastomose oder Pouch) sowie septischen Komplikationen beobachtet [391, 577, 581]. Beide dazu durchgeführten verfügbaren Metaanalysen fanden keinen Einfluss von anti-TNF auf die perioperative Komplikationsrate bei Einschluss aller Operationen [582, 583]. Wenn aber bei letzterer Metaanalyse nur die Patienten, die einen Pouch erhalten hatten, berücksichtigt wurden, bestand doch ein signifikanter Einfluss auf die frühen und späten pouchspezifischen Komplikationen. Diese Korrelation wird durch eine aktuelle Analyse aus einer Datenbank von Versicherungsträgern aus den USA an über 2000 Patienten bestätigt; auch dort waren perioperative Komplikationen mit der Einnahme von Infliximab bis 90 Tage vor der Pouchanlage signifikant assoziiert [584]. Dies ist im Einklang mit den Ergebnissen der beiden größten und statistisch validesten Fall-Kontroll-Studien aus zwei bekannten „high-volume centers“, die beide eine signifikante Assoziation von Anti-TNF-Gabe bis 3 Monate vor restaurativer Proktokolektomie mit perioperativen Komplikationen zeigten [577, 581].

Welches zeitliche Intervall zwischen der letzten Anti-TNF-Medikation und einer planbaren Operation als sicher anzusehen ist, lässt sich auf der Basis der verfügbaren Daten nicht eindeutig sagen. Aus pragmatischer Sicht erscheint ein Intervall von mindestens 4 Wochen angezeigt. Möglicherweise erklären die unterschiedlichen Serumspiegel der TNF-Antikörper bei den jeweiligen Patienten die widersprüchlichen Ergebnisse der verfügbaren Analysen [585]. Inwiefern präoperative Spiegelmessungen sinnvoll sind und dadurch ein sicherer OP-Zeitpunkt definiert werden kann, muss in weiteren Studien untersucht werden.

Die kontinuierliche Einnahme von Azathioprin ist nicht mit einem erhöhten Risiko für postoperative Komplikationen verbun- den. Bezüglich einer immunsuppressiven Therapie mit Calcineurininhibitoren zeigte eine Fallserie bei Kindern keine Unterschiede hinsichtlich postoperativer Komplikationen mit oder ohne präoperative Therapie mit Calcineurininhibitoren [586].

\section{EMPFEHLUNG 5.1.12}

Bei der dreizeitigen Proktokolektomie sollte die Kolektomie bis zum rektosigmoidalen Übergang erfolgen.

Expertenkonsens, Empfehlung, starker Konsens

\section{Hintergrund}

Die hohe Komplikationsrate unter intensivierter medikamentöser Therapie macht das komplikationsärmere dreizeitige Vorgehen erforderlich. Da die Entfernung des Rektums der risikoreichste und für den Patienten am meisten belastende Anteil der Operation ist, wird im ersten Schritt eine subtotale Kolektomie mit Anlage eines endständigen lleostomas durchgeführt. Jedoch sollte berücksichtigt werden, dass möglichst viel entzündungstragendes Kolon entfernt wird. Mit einer Resektion bis zum rektosigmoidalen Übergang sind in der Regel diese beiden Forderungen erfüllt, und die Restproktektomie ist technisch leicht möglich. Eine tiefere Absetzung sollte vermieden werden, weil dadurch das Risiko von Nervenverletzungen in der Folgeoperation deutlich höher ist. Der Verschluss des Rektumstumpfs erfolgt als Blindverschluss nach Hartmann oder unter Belassung des Colon sigmoideum als Ausleitung desselbigen als Schleimfistel im linken Unterbauch. Durch die letztere Variante kann eine etwaige Insuffizienz des Hartmann-Stumpfs vermieden werden, und es besteht die Möglichkeit, in der Zeit zwischen der zweiten und der dritten Operation topisch über die Sigmaschleimfistel mit Kortikoiden oder 5-ASA zu therapieren. Allerdings beinhaltet der partielle Erhalt des Sigmas auch ein Belassen von mehr erkranktem Darm und die Ausleitung desselbigen als zweites Stoma auch eine weitere Schwächung der Bauchwand. Zudem ist das Risiko von Stomakomplikationen (e.g. Stomaausriss) bei diesen Patienten mit schwer entzündlich verändertem Sigma relevant. Daher sollte dieser Ansatz Ausnahmefällen vorbehalten bleiben [587 - 589].

\section{EMPFEHLUNG 5.1.13}

Die Rektumresektion sollte bei benigner Operationsindikation, wenn technisch möglich, darmnah (mesorektumerhaltend) erfolgen, weil dadurch die Komplikationsrate einschließlich Nervenverletzungen verringert werden kann. Evidenzgrad 2, Empfehlungsgrad B, starker Konsens

\section{Hintergrund}

Nach aktueller Datenlage mit einer RCT, einer Fall-Kontroll-Studie und Kohortenstudien sind sowohl die Lebensqualität als auch die Sphinkterfunktion und die Komplikationsraten nach mesorektumerhaltender Resektion besser [590 - 592]. Da die darmwandnahe Präparation auch höchstwahrscheinlich Nervenläsionen vermeidet, erscheint es ratsam, bei benigner Indikation prinzipiell 
darmnah zu resezieren, auch wenn es in Bezug auf die Übersicht, gerade beim engen Becken des Manns, zuweilen schwieriger sein kann.

\section{EMPFEHLUNG 5.1.14}

Bei der ileoanalen Pouchanlage sollte die belassene Rektummukosa nicht länger als $2 \mathrm{~cm}$ sein.

Expertenkonsens, Empfehlung, starker Konsens

\section{Hintergrund}

Die letzten $2 \mathrm{~cm}$ oral der Linea dentata sind funktionell bedeutend und somit für die Lebensqualität der Patienten einflussreich. Innerhalb der letzten $2 \mathrm{~cm}$ oral der Linea dentata befindet sich die anale Transitionalzone, die eine große Rolle für den Erhalt der Nachtkontinenz spielt. Bei der Operationsstrategie muss jedoch berücksichtigt werden, dass postoperativ das Risiko zur Ausbildung einer Rezidiv-/Persistenz-Proctitis ulcerosa besteht.

Da die Erkrankungsschwere, das Beschwerdeausmaß und das Risiko für ein Rezidiv bzw. eine Persistenz mit der Länge der verbliebenen Rektummukosa exponenziell korreliert („Cuffitis“), sollte die Länge der belassenen Rektummukosa $2 \mathrm{~cm}$ nicht übersteigen. Sollte innerhalb der belassenen Rektummukosa eine Entzündung entstehen, besteht die Möglichkeit einer topischen Therapie (siehe Empfehlung 5.2.6).

Hinsichtlich der Anastomosenart scheint die Stapler-Anastomose unter Belassung der analen Transitionalzone für die Funktion tendenziell besser zu sein als die Handnaht, wenn auch prospektive randomisierte Studien mit adäquater Fallzahl zu dieser Fragestellung fehlen. Die bisherige Evidenz ist inkonklusiv, was durch die zwei vorliegenden, qualitativ mäßigen Metaanalysen reflektiert wird [593, 594]. Die kleinere der beiden Analysen bezieht sich explizit nur auf die postoperative Funktion und schloss 4 RCTs ein, wobei sich kein Vorteil für eine der beiden Methoden ergab. Die größere der beiden Analysen untersuchte sowohl Komplikationen als auch funktionelle und Langzeitergebnisse an 4183 Patienten aus 21 Studien, von denen die Mehrheit nicht randomisiert war. Die Komplikationen differierten nicht signifikant, bei den funktionellen Parametern ergaben sich bei der nächtlichen Kontinenz und der Notwendigkeit, Einlagen zu tragen, signifikante Nachteile in der handgenähten Gruppe, allerdings relativierten sich diese, wenn nur die „High-Quality“-Studien analysiert wurden. Hinsichtlich einer Dysplasieentstehung an der analen Transitionszone gab es einen statistischen Trend zu Ungunsten der Staplergruppe. Dieser Befund erscheint logisch, da bei der Staplernaht eben mehr Rest-Rektumschleimhaut verbleibt, die maligne entarten kann. Demgegenüber muss aber berücksichtigt werden, dass auch nach Mukosektomie mit Handnaht Rest-Rektummukosa in Form von Schleimhautinseln zurückbleibt [595].

Jeder Chirurg, der eine restaurative Proktokolektomie durchführt, muss in der Lage sein, bei technischem Versagen einer Stapleranastomose eine transanale Handnaht durchzuführen.

\section{EMPFEHLUNG 5.1.15}

Unter der Indikation einer intraepithelialen Neoplasie oder eines manifesten Karzinoms im Rektum sollte eine komplette Mukosektomie mit Anastomose an der Linea dentata durchgeführt werden.

Evidenzgrad 3, Empfehlungsstärke B, starker Konsens

\section{Hintergrund}

In der Literatur sind mehr als 50 Fälle von „Pouch“-Karzinomen beschrieben. Die Mehrzahl dieser publizierten Pouch-KarzinomFälle geht auf eine belassene Rektummukosa zurück [596, 597]. Daher sollte aus pragmatischen Erwägungen bei bestehender Risikokonstellation in Form von intraepithelialen Neoplasien respektive eines manifesten Karzinoms im Rektum immer radikal die gesamte Rektummukosa entfernt werden. Inwiefern auch bei Vorliegen von Neoplasien im Kolon eine Mukosektomie prinzipiell durchzuführen ist, bleibt kontrovers diskutiert. Eine retrospektive Kohortenstudie aus Kanada an 81 Patienten, bei denen aufgrund einer CU-assoziierten Dysplasie ( $n=52)$ bzw. aufgrund eines Karzinoms ( $n=29$ ) eine RPC mit IAP entweder mittels Hand- oder Staplernaht durchgeführt wurde, ergab für die Staplergruppe $(n=59)$ keine höhere Inzidenz an Pouchkarzinomen und -dysplasien [598]. Zwei Patienten nach Handnaht entwickelten ein Karzinom im Bereich der belassenen Rektummukosa bzw. im Pouch, in der Staplerkohorte fand sich hingegen kein einziger Patient mit Pouch- oder Anastomosenkarzinom. Die Autoren schlussfolgern, dass sich kein prognostischer Vorteil der Mukosektomie mit Handnaht bei Patienten zeigt, die wegen einer Neoplasie operiert werden, und man auch diese mit einer Staplernaht versorgen kann, zumindest wenn man, wie in dieser Studie, nur durchschnittlich einen guten Zentimeter Restmukosa zurücklässt. Letztlich liegen keine Daten vor, die onkologische Vorteile im Langzeitverlauf für die Mukosektomie und Handnaht eindeutig belegen. Entsprechend hat die chirurgische ECCO-Leitlinie auch keine explizite Empfehlung mehr für die generelle Mukosektomie bei der kolorektalen Neoplasie ausgesprochen, sofern nicht das untere Rektum betroffen ist [599]. Dennoch ergab die größte verfügbare Metaanalyse hinsichtlich einer Dysplasieentstehung an der analen Transitionszone einen statistischen Trend zu Ungunsten der Staplergruppe, weswegen die Empfehlung zur Mukosektomie zumindest bei der Rektumneoplasie weiterhin pragmatisch begründet erscheint [593].

\section{EMPFEHLUNG 5.1.16}

Pouchchirurgie sollte nur in dafür spezialisierten Zentren durchgeführt werden.

Evidenzgrad 2, Empfehlungsgrad B, starker Konsens

\section{Hintergrund}

Die restaurative Proktokolektomie mit Ileum-J-Pouch-analer Rekonstruktion ist ein komplexer Eingriff mit flacher Lernkurve, der technisch sowie in der prä- und postoperativen Phase eine 
langjährige Erfahrung und hohe Expertise erfordert [600]. Bezüglich Mortalität, Morbidität und Langzeitpoucherhalt besteht in mehreren Studien eine signifikante Korrelation mit der Zahl der durchgeführten Pouchanlagen des Krankenhauses [601-603]. Für vergleichbar komplexe Eingriffe (Pankreas, Ösophagus) bestehen seit einigen Jahren Mindestmengenregelungen, die in der Regel Mindestmengen von zehn Eingriffen pro Jahr umfassen. Für die ileoanale Pouchchirurgie ist gezeigt worden, dass bei einer Eingriffszahl von über 8 (versus < 8) bzw. über 20 (versus $\leq 5$ ) pro Zentrum pro Jahr die Rate an Pouchversagen signifikant absinkt [555, 602]. Aus pragmatischen Gründen erscheint daher die Forderung nach einer Mindestanzahl von 10 durchgeführten Pouchanlagen pro Jahr pro Zentrum sinnvoll.

\section{EMPFEHLUNG 5.1.17}

Patienten mit einer chronischen Pouchitis oder nach mit Colitis ulcerosa assoziiertem Karzinom oder intraepithelialer Neoplasie sollten jährlich endoskopisch überwacht werden. Evidenzgrad 4, Empfehlungsgrad B, starker Konsens

\section{Hintergrund}

Selbst nach makroskopisch komplett durchgeführter Mukosektomie besteht ein Restrisiko für kleinste verbliebene Schleimhautinseln [595]. Diese Schleimhautinseln können den Ursprung einer intraepithelialen Neoplasie oder eines Karzinoms darstellen [597, 604]. Aus diesem Grund sollte die Patientengruppe, die aufgrund einer intraepithelialen Neoplasie oder eines Karzinoms operiert worden ist, jährlich nachgesorgt werden. Es bestehen zwar keine sicheren Hinweise darauf, dass eine chronische Pouchitis langfristig eine maligne Transformation bedingt, dennoch erscheint es sinnvoll, dass auch für diese Situation postoperativ eine jährliche endoskopische Überwachung erfolgt. Daneben ist für diese besondere Patientengruppe ein enger Arztkontakt zur Verbesserung der Lebensqualität bei vorhandenem Pouch erforderlich.

\section{EMPFEHLUNG 5.1.18}

Die Kolektomie mit ileorektaler Anastomose sollte nur für ausgewählte Konstellationen wie z. B. bei Kinderwunsch empfohlen werden.

Evidenzgrad 3, Empfehlungsgrad B, starker Konsens

\section{Hintergrund}

Proktokolektomien mit ileoanaler Pouchanlage führen sowohl bei Frauen als auch bei Männern zu erhöhter Infertilität. Inwiefern die Fertilitätsrate bei Männern bei darmnaher Rektumdissektion bei benigner Operationsindikation wirklich reduziert ist, ist fraglich (siehe Abschnitt zu darmnaher Dissektion). Eine aktuelle große Registerstudie aus Dänemark an 27379 Patienten zeigt vielmehr, dass im Gegensatz zu Frauen die Geburtenrate bei Männern nach restaurativer Proktokolektomie ansteigt [605]. Bei Patienten mit Kinderwunsch und bestehender Operationsindika- tion (siehe Einschränkung dieser Aussage bei Männern, bei denen rektumnah präpariert wird), bei Frauen auch als Übergangslösung bis zum Abschluss der Familienplanung, sollten eine subtotale Kolektomie mit endständigem Ileostoma und eine lleorektostomie als Alternativen besprochen werden. Grundvoraussetzung für eine Ileorektostomie ist jedoch, dass das belassene Rektum weitestgehend entzündungsfrei und damit auch anastomosenfähig ist. Patienten sollten aber darüber aufgeklärt werden, dass sie in ungefähr $50 \%$ im weiteren Verlauf dann doch restkolektomiert werden müssen und dass die Lebensqualität nach lleorektostomie nicht besser als nach Pouchanlage ist. Eine neuere große Studie aus Frankreich hat zudem gezeigt, dass bei $80 \%$ der Patienten, die vor der Operation unter Therapie mit Immunsuppressiva und Biologika standen (was bei der OP-Indikation „therapierefraktäre Situation " mittlerweile fast immer der Fall ist), innerhalb von 10 Jahren die Ileorektostomie aufgegeben werden musste [606]. Zudem leiden die Patienten nach Ileorektostomie weiterhin häufig an einer Drangsymptomatik, was letztlich auch gerade bei Colitispatienten mit verbliebenem Kolon eines der quälendsten Symptome darstellt $[607,608]$. Eine sekundäre Rektumresektion mit Proktektomie und IPAA kann später mit ähnlich guten Ergebnissen wie bei einer primären Pouchanlage erfolgen [609].

Aufgrund der Seltenheit einer lleorektostomie bei Kindern soll ein spezialisiertes viszeralchirurgisches Zentrum, ggf. in Zusammenarbeit mit Kinderchirurgen, diese Operation bei Kindern durchführen. Die postoperative Betreuung soll in jedem Fall durch oder in enger Kooperation mit Kindergastroenterologen erfolgen.

\section{EMPFEHLUNG 5.1.19}

Das kontinente lleostoma nach Kock kann als mögliche Alternative für besondere Fälle angeboten werden. Evidenzgrad 4, Empfehlungsgrad 0, starker Konsens Es sollte in dafür spezialisierten Zentren durchgeführt werden. Expertenkonsens, Empfehlung, starker Konsens

\section{Hintergrund}

Das kontinente lleostoma nach Kock ist ein technisch komplexes Operationsverfahren mit hoher Komplikationsrate hinsichtlich Funktion und hoher Revisionsrate. Durch das kontinente lleostoma nach Kock kann die Lebensqualität der Patienten im Vergleich zu einem herkömmlichen, nicht kontinenten Ileostoma gesteigert und das Körperempfinden verbessert werden [610 - 612]. Bei Pouchversagen kann, bei ausgeprägtem Wunsch des Patienten, eine Umwandlung in ein kontinentes Ileostoma nach Kock erfolgen [613].

\section{EMPFEHLUNG 5.1.20}

Bei belassenem Rektum unter ileorektaler Anastomose oder bei endständigem Ileostoma mit Rektumblindverschluss nach Hartmann sollte das Intervall der endoskopischen Kontrolle entsprechend der initialen OP-Indikation gewählt werden. Die Durchführung sollte der Empfehlung der allgemeinen koloskopischen Vorsorge bei CU entsprechen.

Evidenzgrad 3, Empfehlungsgrad B, Konsens 


\section{Hintergrund}

Da durch das Belassen des Rektums prinzipiell ein Entartungsrisiko der Rektumschleimhaut weiterhin besteht, ist eine regelmäßige endoskopische Kontrolle mit Biopsien indiziert [614]. Welches Zeitintervall dafür gewählt werden soll, ist durch Studien nicht adäquat geklärt. Aus pragmatischer Sicht erscheinen die in der Nachsorge der Colitis ulcerosa empfohlenen Intervalle sinnvoll (bei Vorliegen von Risikofaktoren für eine neoplastische Entartung siehe Statement 2.31 bzw. > Tab.6, ansonsten symptom- und individuell patientenorientiert). Eine sekundäre restaurative Proktokolektomie mit IPAA sollte prinzipiell mit dem Patienten diskutiert werden oder ggf. auch eine Stumpfentfernung, sofern eine Pouchanlage kontraindiziert oder von dem Patienten nicht gewünscht wird. Auf jeden Fall soll bei Vorliegen der klassischen Risikofaktoren für eine neoplastische Entartung (Dysplasien oder Neoplasien zum Zeitpunkt der Primär-OP, PSC) der Patient über diese chirurgischen Optionen aufgeklärt werden. Zwar sind auch Daten publiziert, die, auch bei Vorliegen derartiger Risikofaktoren, nur eine sehr niedrige Inzidenz neuer Neoplasien im Langzeitverlauf zeigen [615]. Dennoch erscheint bei der verfügbaren, uneindeutigen Datenlage eine regelmäßige Nachsorge nach Ileorektostomie oder Hartmannstumpfanlage sinnvoll, auch weil es sich um unproblematische Untersuchungen mit minimalem Komplikationsrisiko handelt und der Nachteil einer zwar seltenen, aber übersehenen Neoplasie dagegen unverhältnismäßig bedeutsam ist.

\section{EMPFEHLUNG 5.1.21}

In der elektiven Situation ist die laparoskopische restaurative Proktokolektomie der offenen Operation mindestens gleichwertig, in einigen Punkten überlegen. Bei Frauen mit Kinderwunsch sollte bevorzugt minimalinvasiv operiert werden. Evidenzgrad 3, Empfehlungsgrad B, starker Konsens

\section{Hintergrund}

Die laparoskopische restaurative Proktokolektomie mit IPAA ist eine sicher durchführbare Operationsmethode unter der Voraussetzung einer entsprechenden Erfahrung des Zentrums. Bisher konnten, bei vergleichbaren Komplikationsraten, vor allem kosmetische Vorteile gegenüber einer offenen restaurativen Proktokolektomie mit IPAA gezeigt werden [616, 617]. Allerdings dauert die laparoskopische Operation länger und hat daher potenziell auch höhere Prozedurenkosten. Mittlerweile zeigen ein Cochrane Review und 2 neuere „Systematic Reviews“ einige weitere Vorteile im Kurzeitverlauf für die minimalinvasive Gruppe (Wundinfektrate, schnelleres „Ingangkommen“ der Darmtätigkeit) [618 - 620].

Mehrere Fall-Kontroll- und Kohortenstudien legen nahe, dass die Fertilität der Frau durch einen laparoskopischen Ansatz weniger beeinträchtigt wird, vermutlich infolge weniger Adhäsionen [621 -624]. Inwiefern diese Reduktion postoperativer Adhäsionen auch zu weniger lleusepisoden führt, ist nicht belegt [625].

Da die laparoskopische Operation prinzipiell mindestens so gut ist wie die offene Operation, eindeutige kosmetische Vorteile aufweist und bei Frauen die Fertilität besser erhält, sollte sie gerade bei Frauen im gebärfähigen Alter die Methode der Wahl sein.

Bei der dringlichen Operation bzw. auch in der Notfallsituation liegen mehrere Fall-Kontroll-Studien vor, die für den laparoskopischen Zugang vergleichbare Vorteile wie in der elektiven Situation nahelegen [626-628]. Da diese Operationen aber ausnahmslos in spezialisierten Zentren durchgeführt wurden und die meisten Notfalloperationen nicht in derartigen Zentren durchgeführt werden, kann derzeit noch keine generelle Empfehlung für den laparoskopischen Zugang abgegeben werden.

\section{EMPFEHLUNG 5.1.22}

Bei Colitis indeterminata ohne anorektales Fistelleiden und entsprechender Operationsindikation kann eine restaurative Proktokolektomie dem Patienten nach Aufklärung der damit verbundenen Risiken angeboten werden.

Evidenzgrad 3, Empfehlungsgrad 0, starker Konsens

\section{Hintergrund}

Bei ungefähr $7 \%$ der Patienten mit einer Colitis kann keine exakte Diagnose gestellt werden, um Morbus Crohn von Colitis ulcerosa zu unterscheiden [629]. In der Literatur finden sich Berichte über ein schlechteres Langzeitergebnis nach restaurativer Proktokolektomie mit IPAA bei Patienten mit Colitis indeterminata. Diesen stehen jedoch Berichte gegenüber, in denen Patienten mit Colitis indeterminata kein schlechteres Langzeitergebnis haben als Patienten mit Colitis ulcerosa [630, 631]. Die sekundäre Diagnose eines Morbus Crohn nach stattgehabter restaurativer Proktokolektomie mit IPAA ist oftmals mit Komplikationen, insbesondere einer erhöhten Rate an Pouchversagen, behaftet. Allerdings relativiert sich dies, wenn man nur die Patienten betrachtet, die präoperativ eine isolierte Colitis ohne Fisteln und ohne Dünndarmbefall aufwiesen [632]. Trotz dieser widersprüchlichen Datenlage kann eine restaurative Proktokolektomie mit IPAA - nach ausführlicher Diskussion mit dem Patienten durchgeführt werden.

Bei Kindern und Jugendlichen liegt der Anteil der Patienten mit Colitis indeterminata bei bis zu 22 \% [633]. Im Verlauf kann bei einem Großteil der Patienten eine diagnostische Zuordnung zu Colitis ulcerosa oder Morbus Crohn gelingen, sodass bei Kindern mit operationsbedürftiger Colitis indeterminata ein dreizeitiges Vorgehen sinnvoll ist.

\subsection{Pouchitis}

\section{EMPFEHLUNG 5.2.1}

Die Diagnose Pouchitis sollte unter Berücksichtigung der Parameter Klinik, Endoskopie und Histologie erfolgen. Evidenzgrad 5, Empfehlungsgrad B, starker Konsens 


\section{Hintergrund}

Die Pouchitis wird als Entzündung im Pouch nach Ausschluss operativer Komplikationen oder anderer sekundärer Ursachen definiert. Es sollten dabei verschiedene Verlaufsformen unterschieden werden. Die Einteilung soll anhand des zeitlichen Verlaufs in akute, akut-rezidivierende und chronische Pouchitis erfolgen. Andere Unterteilungen können nach dem klinischen Verlauf unternommen werden: antibiotikasensitive oder -refraktäre akute Pouchitis, rekurrierende und chronische Pouchitis (z. B. > 3 Monate) $[634,635]$.

Die Diagnose einer akuten Pouchitis wird auf der Basis der klinischen Symptomatik (Stuhlfrequenz, Blutung, Fieber, Schmerzen), ergänzt durch Endoskopie (Rötung, Ödem, Erosionen, Ulzerationen, Spontaneinblutungen, Fibrinbeläge), Histologie (Ulzerationen, Kryptenabszesse, Infiltration durch Entzündungszellen) und der klinischen, insbesondere rektal-digitalen Untersuchung gestellt [636]. Die akut-rezidivierende Pouchitis ist durch wiederholt auftretende akute Schübe gekennzeichnet. Die Diagnose einer chronischen Pouchitis ergibt sich durch eine entzündliche Reaktion im Pouch, deren Klinik und endoskopischer/histologischer Befund über mehr als 3 Monate anhält. Das Risiko einer akuten Pouchitis liegt bei Patienten nach restaurativer Proktokolektomie mit IPAA bei Colitis ulcerosa bei etwa $30 \%$ in den ersten zwei Jahren nach Operation. Im Lauf der Nachbeobachtungszeit steigt das Risiko langsam auf bis über $50 \%$ an [637 - 643]. Bei ungefähr $5 \%$ der Patienten mit einer Pouchitis geht die akute Verlaufsform in eine chronische über. Risikofaktoren für eine Pouchitis sind bestehende extraintestinale Manifestationen der CU. Dabei ist insbesondere die PSC zu erwähnen [643-647]. Zusätzlich gehen anhaltend hohe Entzündungsaktivität und präoperative Backwashileitis mit einer erhöhten Rate an Pouchitis einher. Die Parameter zur Diagnose der akuten Pouchitis werden durch den Pouchitis Disease Activity Index (PDAI) zusammengefasst [636]. Wenn sich trotz entsprechender klinischer Symptomatik nach endoskopischen, bildgebenden und histologischen Kriterien keine Ursache für eine Pouchitis findet und andere Krankheiten ausgeschlossen wurden, kann die Diagnose eines irritablen Pouchsyndroms gestellt werden [648].

\section{EMPFEHLUNG 5.2.2}

Bei einer chronischen Pouchitis sollte ein Morbus Crohn, eine chirurgische Komplikation oder eine Infektion ausgeschlossen werden.

Evidenzgrad 5, Empfehlungsgrad B, starker Konsens

\section{Hintergrund}

Bei Diagnosestellung Pouchitis sind Untersuchungen zum Ausschluss von sekundären Pouchitisformen empfohlen. Zum Ausschluss einer chirurgischen Ursache einer Pouchitis sind Computertomografie, Kernspintomografie, Kontrasteinlauf und Endosonografie notwendig und geeignet [649-652]. Gegebenenfalls sind diese Untersuchungsmethoden im Verlauf zu wiederholen. Das Vorliegen eines Morbus Crohn, von Fisteln oder Abszessen, einer Anastomoseninsuffizienz, einer Ischämie oder einer oppor- tunistischen Infektion sollte ausgeschlossen werden. Für einen Morbus Crohn spricht neben der Histologie eine Beteiligung des Prä-Pouch-Ileumsegments (zuführende Schlinge zum Pouch).

\section{EMPFEHLUNG 5.2.3}

Als Primärtherapie der akuten Pouchitis sollen Ciprofloxacin oder Metronidazol eingesetzt werden.

Evidenzgrad 1, Empfehlungsgrad A, starker Konsens

Bei Versagen der Monotherapie kann auch eine Kombination eingesetzt werden. Evidenzgrad 3, Empfehlungsgrad 0, Konsens

Antibiotikarefraktäre Verlaufsformen sollten mit oralem oder lokalem Budesonid behandelt werden.

Evidenzgrad 3, Empfehlungsgrad B, starker Konsens

Weitere Therapieoptionen können unter anderem Infliximab, Adalimumab, Vedolizumab, Ustekinumab oder Calcineurininhibitoren sein.

Expertenkonsens, Empfehlung offen, Konsens

\section{Hintergrund}

Es gibt mehrere randomisierte kontrollierte Studien zur medikamentösen Therapie der Pouchitis [653 - 656]. Die Empfehlung zur Therapie der akuten Pouchitis mit Antibiotika (Metronidazol, Ciprofloxazin) wird durch mehrere, allerdings kleine Studien gestützt [657 - 660]. Dabei könnte Ciprofloxacin der Therapie mit Metronidazol überlegen sein [656]. Auch eine Kombinationstherapie ist möglich [661, 662]

Zu Dosis und Dauer der Antibiotikatherapie liegen keine gesicherten Daten vor, sie müssen individuell getestet werden. Üblich ist eine orale Therapie mit Ciprofloxazin $2 \times 250 \mathrm{mg}$ bis $2 \times 500 \mathrm{mg} / \mathrm{d}$ für 2 Wochen oder Metronidazol $2-3 \times 400 \mathrm{mg}$. Bei Unverträglichkeit von oralem Metronidazol stellt topisch angewendetes Metronidazol (Suppositorien) eine Alternative dar [663]. Für Rifaximin kann keine Empfehlung ausgesprochen werden [664].

Bei antibiotikarefraktärer Pouchitis kann ein Versuch mit topischem Budesonid unternommen werden [665]. Die lokale Budesonid-Therapie ist der lokalen Gabe von Metronidazol gleichwertig, scheint aber etwas besser verträglich zu sein [666].

Eine Behandlung mit TNF-Antagonisten und/oder Immunsuppressiva ist möglich. In einem kürzlich erschienenen Review wurden 19 Publikationen mit insgesamt 192 Patienten diesbezüglich ausgewertet [667]. Indikationen für eine Anti-TNF-Therapie waren antibiotikarefraktäre Verlaufsformen, fistulierende Verläufe und inflammatorische, stenosierende Pouchitisformen. Basierend auf den 3 größten eingeschlossenen Studien $(n=87)$, erreichte die Therapie mit Infliximab ein kombiniertes partielles und komplettes Ansprechen von 84 - $88 \%$ nach 6-10 Wochen und von 45 $58 \%$ nach 52 Wochen [667].

In einer retrospektiven kanadischen Studie wurde der Verlauf von 152 Patienten mit einer therapieresistenten Pouchitis analysiert. 42 davon wurden mit Infliximab behandelt. Das Post-Induktionsansprechen wurde in $74 \%$ der Fälle erreicht und ein anhaltendes Ansprechen in 62,6\% der Fälle. Der mittlere PDAI und das CRP 
zeigten unter der Therapie einen statistisch signifikanten Rückgang [668].

Der Effekt von Adalimumab konnte in einer Fallserie an 48 Patienten gezeigt werden. Dabei betrug das kombinierte partielle und komplette Ansprechen $71 \%$ und 54\% nach einem medianen Beobachtungszeitraum 8 bzw. 25 Wochen [669]. Bei Patienten mit einer therapierefraktären Pouchitis und einem InfliximabTherapieversagen konnte zu Woche 52 in $50 \%$ der Fälle durch eine Adalimumab-Zweitlinientherapie ein permanentes Ileostoma verhindert werden [670].

Weitere Therapieoptionen sind unter anderem Vedolizumab, Ustekinumab oder Calcineurininhibitoren, wobei die Studien dazu bei einer akuten, antibiotikarefraktären Pouchitis durchgeführt wurden und es sich um retrospektive Daten und Fallserien mit heterogenen Patientenkollektiven handelt [653, 671 - 673].

\section{EMPFEHLUNG 5.2.4}

Bei einer häufig rekurrierenden oder einer chronischen Pouchitis sollte intermittierend eine Monotherapie oder eine kombinierte antibiotische Therapie mit Ciprofloxacin und/ oder Metronidazol eingesetzt werden.

Evidenzgrad 3, Empfehlungsgrad B, starker Konsens

Antibiotikarefraktäre Verlaufsformen sollten mit oralem oder lokalem Budesonid behandelt werden.

Evidenzgrad 3, Empfehlungsgrad B, Konsens

Weitere Therapieoptionen sind unter anderem Infliximab, Adalimumab, Vedolizumab, Ustekinumab, Rifaximin, Calcineurininhibitoren oder Alicaforsen.

Evidenzlevel 4, Empfehlungsgrad 0, Konsens

\section{Hintergrund}

Bei fehlendem Ansprechen einer Monotherapie mit Metronidazol oder Ciprofloxazin kann eine orale Kombinationstherapie aus Ciprofloxazin und Metronidazol oder eine orale Kombinationstherapie aus Ciprofloxazin und Rifaximin eingesetzt werden [657, 662]. Die Datenlage dazu ist aber schwach.

In einem kürzlichen Review wurde der Stellenwert der verschiedenen medikamentösen Therapieoptionen für die chronische Pouchitis nach IPAA bei CU untersucht [653]. Dabei wurden 21 Manuskripte eingeschlossen. Es zeigte sich, dass Antibiotika in $74 \%$ eine Remission (95\%-KI: 56 - $93 \%$ ), ( $<<0,001$ ) erreichen können. TNF-Antikörper erreichten Remissionsraten von $53 \%$ (95\%-KI: $30-76 \%),(p<0,001)$. Therapien mit Steroiden, Bismuth, Elementardiät oder Tacrolimus erreichten ebenfalls eine allerdings nicht signifikante Remission. Zur lokalen Therapie mit Tacrolimussuppositorien gibt es Einzelfallberichte. Die Datenlage zum Effekt einer FMT bei Pouchitis ist für eine Empfehlung noch unzureichend und konnte keine Remissionsinduktion zeigen $[674,675]$.

Weitere Therapieoptionen sind unter anderem Infliximab, Adalimumab, Vedolizumab, Ustekinumab, Rifaximin, Calcineurininhibitoren oder Alicaforsen [653, 667, 671 - 673, 676-679].
Zu der Bakterienformulierung VSL\#3 liegen ältere Studien vor, die eine Wirksamkeit in der Behandlung der Pouchitis gezeigt haben. Eine gepoolte Analyse von zwei Studien (76 Teilnehmer) legt nahe, dass VSL\#3 wirksamer als Plazebo für die Aufrechterhaltung der Remission ist [680-682]. 85 \% (34/40) der Patienten konnten mit VSL\#3 ihre Remission über 9 - 12 Monate aufrechterhalten, im Gegensatz zu der Plazebogruppe, in der dies nur bei $3 \%$ (1/36) der Fall war (RR 20,24, 95\%-KI 4,28-95,81). Eine GRADEAnalyse zeigte aber, dass, aufgrund der geringen Zahl an Ereignissen die Evidenzstärke dieser Daten gering ist [656].

Perianale Entzündungen und Reizungen stellen ein großes Problem vieler Pouchträger dar. Eine entsprechende Anamnese, eine Untersuchung und ggf. ein Abstrich können oft die entsprechende Diagnose stellen. Abduschen nach dem Stuhlgang oder Feuchttücher ohne Alkohol, Parfüm und Farbstoffe, eine optimale Hautpflege, z. B. mit Panthenol oder Zink, eine Ernährungsumstellung oder möglicherweise der Einsatz von Cholestyramin können zur Wundheilung beitragen.

\section{EMPFEHLUNG 5.2.5}

Bei Vorliegen von Risikofaktoren sollten jährlich endoskopische Kontrolluntersuchung erfolgen. Andernfalls sollte bei Beschwerden oder spätestens alle 2 Jahre eine Pouchoskopie erfolgen.

Expertenkonsens, Empfehlung, Konsens

\section{Hintergrund}

Diese Empfehlung stützt sich nicht auf spezifische Studien in der Literatur. Basierend auf den Erfahrungen der Überwachungsstrategien vor restaurativer Proktokolektomie mit IPAA erscheint eine jährliche endoskopische Kontrolluntersuchung sinnvoll, obwohl das Pouch-Karzinom eine seltene Entität darstellt [683].

Ob das Überwachungsintervall sich bei Patienten, bei denen eine Pouchanlage aus nicht-malignen Gründen erfolgt ist, länger sein kann, ist unklar. Patienten mit einem Pouch-Karzinom hatten in der Regel bereits ein Karzinom im Kolon oder Rektum, das zur restaurativen Proktokolektomie mit IPAA geführt hat. Pouch-Karzinome entstehen in der Regel aus verbliebener Schleimhaut des anorektalen Übergangs, sodass bei einem Karzinom im unteren Rektum aus pragmatischer Sicht eine komplette Mukosektomie im Rahmen der restaurativen Proktokolektomie zu bevorzugen ist (s. auch Statement 5.1.15) [604, 684].

\section{EMPFEHLUNG 5.2.6}

Bei einer Cuffitis sollte initial ein Therapieversuch mit topischer 5-ASA-Applikation durchgeführt werden.

Evidenzgrad 3, Empfehlungsgrad B, starker Konsens

Bei therapierefraktärer Entzündung kann eine endoskopische Mukosektomie oder Rest-Proktektomie erwogen werden. Expertenkonsens, Empfehlung, Konsens 


\section{Hintergrund}

Eine Cuffitis, vor allem nach einer „double-stapled IPAA“, kann eine Pouchdysfunktion mit Symptomen verursachen, die eine Pouchitis oder ein irritables Pouchsyndrom (IPS) imitieren können.

Im Gegensatz zu einem IPS, das natürlich koexistieren kann, sind perianale Blutungen ein charakteristisches Merkmal einer Cuffitis. Die Endoskopie durch einen erfahrenen Untersucher und die histologische Beurteilung der Epithelmanschette zwischen der Linea dentata und der pouchanalen Anastomose kann die Diagnose stellen [685].

In einer Open-Label-Studie mit 14 konsekutiven Cuffitis-Patienten wurde der Effekt von Mesalazin-500-mg-Suppositorien blind untersucht. Dabei konnte Mesalazin gegenüber Plazebo signifikant den Gesamt-Cuffitis-Aktivitätsindex sowie die Symptom-, Endoskopie und Histologie-Subscores reduzieren. 92 \% der Patienten mit blutigen Stuhlgängen und $70 \%$ der Patienten mit Arthralgie (einem charakteristischen klinischen Merkmal der Cuffitis) haben auf eine topische Mesalazin-Therapie angesprochen. Systemische oder topische Nebenwirkungen wurden nicht berichtet [686].

\section{EMPFEHLUNG 5.2.7}

Patienten mit einem irritablen Pouchsyndrom können wie Patienten mit RDS behandelt werden.

Expertenkonsens, Empfehlung offen, Konsens

\section{Hintergrund}

Im Gegensatz zu dem Behandlungsansatz zur Behandlung des Reizdarmsyndroms gibt es keinen Algorithmus für das Management des IPS. Bei Patienten mit IPS können, wie beim Reizdarmsyndrom, symptomatische Therapien wie z. B. diätetische Modifikationen, Ballaststoffe, Loperamid, krampflösende Medikamente und Antidepressiva eingesetzt werden [648, 687].

\section{Ernährung und komplementäre Verfahren}

\subsection{Ernährung in der Ätiologie und Prävention der Colitis ulcerosa}

\section{EMPFEHLUNG 6.1.1}

Mit Ausnahme des Stillens gibt es keine Ernährungsfaktoren, die zur Risikominderung für ein CU empfohlen werden könnten. Die Stilldauer sollte mindestens 6 Monate betragen. Evidenzgrad 3, Empfehlungsgrad B, Konsens

\section{Hintergrund}

Die Beobachtung, dass CU bis Ende der 1950er-Jahre extrem selten waren und seit dieser Zeit in allen westlichen Industrieländern erheblich zugenommen haben, veranlasste zur Annahme, dass Änderungen in der Ernährung eine mögliche Ursache für die
Häufigkeitszunahme sind. Basierend auf retrospektiven Studien wurden der nach Kriegsende angestiegene Konsum an raffinierten Kohlenhydraten, chemisch aufbereiteten Fetten, die Abnahme des Verzehrs an Ballaststoffen sowie allergische Reaktionen auf Bäckerhefe angeschuldigt. Neuere prospektive Studien zeigten eine mögliche Assoziation des Auftretens von CU mit dem vermehrten Verzehr von tierischem Eiweiß [688] sowie gesättigten und Transfettsäuren [682, 689]. Demgegenüber findet sich für das Auftreten einer CU eine signifikant inverse Relation zum täglichen Verzehr von Gemüse, Omega-3-Fettsäuren [690] und Vitamin D [691].

Zwei systematischen Reviews aus den Jahren 2004 und 2009 zufolge lässt sich lediglich für das Stillen eine signifikante Risikominderung (23\%) für das spätere Auftreten einer CU belegen [692, 693]. Dies wurde in zwei nachfolgenden Kohortenstudien aus Neuseeland [694] und Dänemark [695] bestätigt. Die Stilldauer sollte mindestens 6 Monate betragen, wenn möglich auch länger [696].

\section{Mangelernährung}

\section{EMPFEHLUNG 6.1.2}

Patienten mit CU haben ein erhöhtes Risiko für eine Mangelernährung. Sie sollten daher zum Zeitpunkt der Erstdiagnose und im weiteren Krankheitsverlauf auf das Vorliegen einer Mangelernährung untersucht werden.

Evidenzlevel 2, Empfehlungsgrad B, starker Konsens

\section{Hintergrund}

Da die durch Mangelernährung hervorgerufenen Komplikationen den Patienten oftmals mehr schwächen können als der zugrunde liegende entzündliche Prozess selbst, sind Prävention und Behandlung der Malnutrition obligatorische Bestandteile der Therapie chronisch-entzündlicher Darmerkrankungen.

Die Prävalenz der Unterernährung liegt bei Erwachsenen mit aktiver CU bei 7,2\% (versus 1,8\% in gesunden Kontrollen), was einem 5-fach höheren Risiko entspricht (OR 5,57; $95 \%$-KI: 5,29$5,86)$. Das damit verbundene Risiko von Komplikationen (schwerer Verlauf, längere Liegezeiten) sowie die daraus resultierenden Kosten sind ebenfalls erhöht [697].

Bis zu 85 \% der Kinder mit CED weisen Zeichen einer Mangelernährung auf (Morbus Crohn > Colitis ulcerosa), wovon 15-40\% im Wachstum retardiert sind [696]. Etwa 25 - 80 \% weisen erniedrigte Albuminspiegel auf. In Abhängigkeit von Krankheitsaktivität und -dauer liegt bei 20-85\% der Patienten eine negative Stickstoffbilanz vor. Exzessive Proteinverluste über die entzündete Darmmukosa - mittels fäkalen $\alpha_{1}$-Antitrypsins bestimmt - scheinen dabei der Hauptverursacher der Gesamtkörperproteindepletion zu sein. Eine Proteinmalabsorption könnte zusätzlich dazu beitragen. Die negative Stickstoffbilanz kann außerdem auf dem katabolen Effekt von Medikamenten wie Antibiotika und Steroiden beruhen [698]. 


\section{EMPFEHLUNG 6.1.3}

Bei Patienten mit CU besteht ein erhöhtes Risiko für einen Mikronährstoffmangel. Sie sollten daher initial und im weiteren Krankheitsverlauf, wenn klinische Zeichen oder erhöhte Risiken für einen Mangel vorliegen, auf das Vorliegen eines Mangels an Mikronährstoffen untersucht werden. Evidenzgrad 2, Empfehlungsgrad B, starker Konsens

\section{Hintergrund}

In Abhängigkeit von Patientenkollektiv, Krankheitsaktivität sowie gewähltem Biomarker und Cut-off-Wert findet sich ein Eisenmangel bei $30-70 \%$, ein 25-OH-Vitamin D-Mangel bei $40-60 \%$, ein Folsäuremangel bei 5-10\% und ein Zink- bzw. Selenmangel bei jeweils 20 - 30 \% der Patienten. Demgegenüber ist das Auftreten eines Vitamin- $B_{12}$-Mangels nur nach Anlage eines IAP beschrieben [698-702]. Die Ätiologie des Vitamin- $B_{12}$-Mangels bei IAP ist multifaktoriell, diskutiert werden eine verminderte Resorptionsleistung sowie die fast regelhaft auftretende bakterielle Fehlbesiedlung [703].

\section{Durchführung der Ernährungs-/Substitutionstherapie}

\section{EMPFEHLUNG 6.1.4}

Eine spezielle enterale Ernährungstherapie und/oder ausschließliche parenterale Ernährung sollte als primäre Therapie zur Remissionsinduktion bei Colitis ulcerosa nicht durchgeführt werden.

Expertenkonsens, Empfehlung, starker Konsens

\section{Hintergrund}

Im Gegensatz zu Morbus Crohn ist der Einfluss ernährungstherapeutischer Maßnahmen (Trinknahrung, enterale und parenterale Ernährung) auf die Krankheitsaktivität in der akuten Phase oder bei chronisch aktiver Colitis ulcerosa wenig oder nicht belegt [704]. Wie bei allen Erkrankungen gelten auch bei chronisch-entzündlichen Darmerkrankungen die ernährungs-therapeutischen Grundsätze einer Stufentherapie: Ernährungsberatung, zusätzliche Ernährung durch Trinknahrung, Sondennahrung, parenterale Ernährung [704]. Bei der Behandlung von Kindern sollte allerdings berücksichtigt werden, dass eine alleinige Ernährungsberatung/ Diätberatung nicht effektiv zur Behandlung einer Mangelernährung und/oder spezifischer Mangelzustände bzw. zur Behandlung des Wachstumsrückstands ist [704].

Eine enterale Ernährung (total oder partiell) soll bei CU als supplementäre Therapie durchgeführt werden, um in der akuten Phase der Erkrankung eine adäquate Zufuhr an Nährstoffen zu gewährleisten, insbesondere wenn schon Zeichen einer Mangelernährung vorhanden sind oder ein hohes Risiko für Ernährungsdefizite besteht [345].

Trotzdem kann die total parenterale oder enterale Ernährung insbesondere bei fulminanter Colitis ulcerosa mit einem Standardregime zur Sicherstellung einer adäquaten Nährstoffversorgung indiziert sein. Der Einfluss spezifischer Substrate wie Omega-3-
Fettsäuren, Glutamin oder Butyrat auf die Krankheitsaktivität ist bei Colitis ulcerosa nicht belegt [705-709].

Solange keine Gewichtsabnahme besteht, liegt der Energiebedarf zwischen 25 und $30 \mathrm{kcal} / \mathrm{kg}$ KG/Tag [710]. Der Eiweißbedarf ist infolge der katabolen Stoffwechsellage im Rahmen der Entzündung sowie des etwaigen intestinalen Eiweißverlustes erhöht und beträgt $1-1,5 \mathrm{~g} / \mathrm{kg} \mathrm{KG} / \mathrm{Tag}$, bei septischen oder schwerst mangelernährten Patienten bis zu $2 \mathrm{~g} / \mathrm{kg} \mathrm{KG} / \mathrm{Tag}$ [710]. Eine alleinige total parenterale Ernährung (TPE) sollte bei CU nur noch zur Korrektur einer schweren Mangelernährung durchgeführt werden, insbesondere vor einer Operation, oder bei Komplikationen, wenn bei einem komplizierten Verlauf mit enteraler Ernährung nur unzureichend Kalorien verabreicht werden können oder die enterale Ernährung nicht durchführbar ist [711, 712].

\section{EMPFEHLUNG 6.1.5}

Eine spezielle Diät oder Ernährungstherapie zur Remissionserhaltung sollte nicht empfohlen werden.

Expertenkonsens, Empfehlung, starker Konsens

\section{Hintergrund}

Zwar zeigen kleinere z.T. unkontrollierte Studien für mit Omega-3-Fettsäuren angereicherte Supplementnahrungen einen remissionserhaltenden Effekt bei Patienten mit Morbus Crohn, eine generelle Therapieempfehlung bei Colitis ulcerosa kann daraus jedoch derzeit nicht abgeleitet werden [713]. Gleiches gilt für die von Roediger Mitte der 1990er für CU-Patienten vorgeschlagene sulfitarme Kost, die im Meiden von Lebensmitteln besteht, die reich sind an schwefelhaltigen Aminosäuren [709, 714].

\section{EMPFEHLUNG 6.1.6}

Ein isolierter Mikronährstoffmangel sollte durch entsprechende orale oder parenterale Supplemente behandelt werden. Eine generelle Vitamin- oder Spurenelementsubstitution ist bei CU nicht sinnvoll.

Expertenkonsens, Empfehlung, starker Konsens

\section{Hintergrund}

Bei nachgewiesenem Mikronährstoffmangel sollte die Vitaminund/oder Spurenelementsubstitution gezielt bei Vorliegen eines Mangels nach den entsprechenden Empfehlungen erfolgen [702, 715, 716]. In der Remissionsphase kann die Substitution primär oral durchgeführt werden. Bei Unverträglichkeit oraler Präparate (z. B. Eisen) oder unzureichender Resorption (Eisen, Vitamin $\mathrm{B}_{12}$ ) insbesondere bei ausgeprägter entzündlicher Aktivität ist eine parenterale Substitution erforderlich.

Eine Eisensupplementierung sollte auf jeden Fall erfolgen, wenn eine manifeste Anämie besteht. Eisenmangel ohne manifeste Anämie erfordert ein individualisiertes Vorgehen. Die Entscheidung über den Zeitpunkt und die Art der Therapie wird dabei von Symptomatik, Ätiologie, Schweregrad, Dynamik des Hb-Abfalls, Komorbidität und Risiken der Therapie bestimmt [717]. 
Behandlungsziele bei Eisenmangelanämie sind die Anhebung des Hb-Werts um mehr als $2 \mathrm{mg} / \mathrm{dl}$ oder auf normale Werte innerhalb von 4 Wochen sowie die Erhöhung des Ferritinspiegels auf $>100 \mu \mathrm{g} / \mathrm{l}$. Die allenthalben empfohlene Berechnung der notwendigen Eisenmenge nach Ganzoni (Gesamteisendefizit $(\mathrm{mg})=$ [Soll$\mathrm{Hb}$ - Patienten-Hb $(\mathrm{g} / \mathrm{dl})] \times$ Körpergewicht $(\mathrm{kg}) \times 2,4$ + Reserveeisen $(\mathrm{mg})$ ) zeigt sich zum einen im Routinealltag als „unhandlich“, zum anderen wird zumindest bei CED-Patienten die notwendige Eisenmenge unterschätzt [718]. Als einfacher und zudem effektiver erweist sich die kürzlich publizierte „FERGIcor“-[719].

Grundsätzlich stehen zwei Applikationswege zur Eisensubstitution zur Verfügung, die orale Gabe von geeigneten Eisen(II, III)Verbindungen oder die intravenöse Applikation von Eisen(III)Komplexen [718].

Bei leichtgradiger Eisenmangelanämie (Hb 11 - 13g/dl) kann mit einer oralen Eisenzufuhr von ca. 100 mg/d für 4 Wochen begonnen werden. Bei Intoleranz oder unzureichendem Hb-Anstieg, d. h. weniger als $2 \mathrm{~g} / \mathrm{dl}$, sollte rechtzeitig auf eine intravenöse Eisentherapie umgestellt werden; wegen der begrenzten oralen Resorption von Eisen sollte bei schwerer Anämie in jedem Fall eine intravenöse Applikation mit dem Ziel der Hb-Wert-Normalisierung und Auffüllung der Eisenspeicher erfolgen. Eine absolute Indikation für eine intravenöse Eisentherapie sind [717, 718]:

- schwere Anämie (Hb-Wert < $10 \mathrm{~g} / \mathrm{dl}$ )

- Unverträglichkeit von oralem Eisen oder unzureichender

Anstieg innerhalb der ersten 2 Wochen

- ausgeprägte Krankheitsaktivität

- begleitende Therapie mit Erythropoetin stimulierenden Substanzen

- entsprechende Patientenpräferenz

\section{Therapie des Zinkmangels}

Mit Durchfall (Stuhlvolumina $>300 \mathrm{~g} / \mathrm{d}$ ) bzw. Stoma-Output gehen erhebliche Mengen Zink verloren, wobei pro Liter StomaOutput mit einem Verlust von 12 mg elementarem Zink zu rechnen ist. Dies liegt deutlich über dem normalen Zinkbedarf und über dem Gehalt üblicher Spurenelementpräparate [720, 721]. Bei Vorliegen eines Zinkmangels sollten 30 bis $45 \mathrm{mg}$ Zink in Form von Zink-Histidin oder -Glukonat oral (ca. 1 Std. vor dem Frühstück) eingenommen werden [722]. Da Zink mit der intestinalen Eisen-, v. a. Kupferresorption interferiert, sollte eine orale Supplementierung nicht länger als 2 - 3 Wochen durchgeführt werden [723], bei längerfristiger Einnahme sollte pro $8-15 \mathrm{mg}$ elementares Zink $1 \mathrm{mg}$ elementares Kupfer substituiert werden. Bei (oftmals) nicht ausreichendem Ansprechen sollte Zink parenteral substituiert werden (z. B. Zink-Aspartat als Mono-Injektion oder als Infusionszusatz bis $5 \mathrm{mg} / \mathrm{Tag}$ ) [721, 724].

\section{Therapie des Vitamin- $\mathrm{B}_{12}$-Mangels}

Aufgrund der nur geringen Resorption von 1 - 3 \% von Cyanocobalamin wird in der Regel die parenterale Applikation bevorzugt. Leider sind die Therapieempfehlungen hinsichtlich der Dosierung und Anwendung der $\mathrm{B}_{12}$-Substitution weithin uneinheitlich und meist unterdosiert. Derzeit existieren zwei medikamentöse Vitamin- $B_{12}$-Präparationen: Cyanocobalamin und Hydroxycobalamin. Im Hinblick auf eine ausreichende Dosierung ist zu beachten, dass erst dann klinische Ausfallerscheinungen auftreten, wenn der Vitamin- $B_{12}$-Speicher des Körpers ( $4-5 \mathrm{mg}$ ) auf 5 bis $10 \%$ reduziert ist. Das heißt, Ziel einer adäquaten Behandlung muss es sein, dieses Defizit auszugleichen. Um die leeren Körperdepots so schnell wie möglich aufzufüllen, wird daher folgende Vorgehensweise empfohlen: Man verabreicht in der 1. Woche an fünf Tagen $1000 \mu \mathrm{g}$ Hydroxycobalamin i. m./s. c. (davon werden etwa $45 \%$, d. h. $450 \mu \mathrm{g}$ aufgenommen - gegenüber nur $16 \%$ bei Cyanocobalamin). Im Folgemonat werden $1000 \mu \mathrm{g}$ wöchentlich injiziert (alternativ täglich $500 \mu \mathrm{g}$ Hydroxycobalamin an fünf Tagen der Woche über einen Monat). Zur Überwachung der Vitamintherapie erweist sich die Messung der Homozystein- bzw. MMA-Spiegel als hilfreich [718].

\section{Therapie des Vitamin-D-Mangels}

Bezüglich des Zielspiegels für 25-OH-Vitamin D sowie der dafür benötigten Dosierung bestehen unterschiedliche Auffassungen [725]. Die Endocrine Society empfiehlt bei Erwachsenen eine tägliche Vitamin-D-Zufuhr von 1500 - $2000 \mathrm{IE}$ pro Tag bei einem gewünschten Vitamin-D-Spiegel von > $75 \mathrm{nmol} / \mathrm{l}$ (>30 ng/ml). In bestimmten Situationen, beispielsweise bei Adipositas, bei Einnahme von Antikonvulsiva oder unter Glukokortikoidtherapie, kann diese Dosis verdoppelt oder sogar verdreifacht werden. Gerade unter einer Glukokortikoidtherapie kann der Vitamin-DSpiegel sinken, sodass er entweder kontrolliert werden sollte oder täglich $1000 \mathrm{IE} 25-\mathrm{OH}$-Vitamin D supplementiert werden sollten [726, 727]. Evidenzbasierte Empfehlungen über Zielspiegel, Dosierung und Dauer einer Vitamin-D-Supplementierung für CED-Patienten fehlen. Garg et al. empfehlen je nach Serumspiegel 1000 - 5000 IU/Tag mit einem Multiplikator von 1,5 - 20 bei Morbus-Crohn-Patienten mit Dünndarmbefall und/oder Adipositas. Die $25-\mathrm{OH}$-Vitamin-D-Spiegel sollten dauerhaft $>30 \mathrm{ng} / \mathrm{ml}$ (75 mmol/L) liegen. Um eine möglichst schnelle Normalisierung der 25-OH-Spiegel zu erzielen (innerhalb von 2 Wochen), schlagen Van Groningen et al. eine initiale Aufsättigungsdosierung $(\mathrm{IU})=40 \times\left(75-\right.$ Serum-25- $\left.\mathrm{OHD}_{3}\right) \times \mathrm{KG}$ vor [728]. Die errechnete Dosis wird an 3 Wochentagen (Mo/Mi/Fr) zu jeweils $20000 \mathrm{IU}$, gefolgt von einer einwöchigen Erhaltungsdosis mit $20000 \mathrm{IU}$ verabreicht. Generell sollte Cholecalciferol (Vitamin D3) aufgrund der besseren Bioverfügbarkeit gegenüber Ergocalciferol (Vitamin D2) bevorzugt werden [729].

Bei einer Einnahme von bis zu 4000 IE Vitamin D3 pro Tag ist eine Kontrolle des Vitamin-D-Spiegels nicht regelhaft erforderlich. Eine Vitamin-D-Intoxikation ist erst bei dauerhaften Spiegeln $>150 \mathrm{ng} / \mathrm{ml}$ (> $375 \mathrm{nmol} / \mathrm{l})$ zu erwarten [730, 731].

\section{Chirurgische Aspekte der Ernährung bei CU}

EMPFEHLUNG 6.1.7

Präoperativ sollte bei schwerer Mangelernährung (BMI < 19, Gewichtsverlust von mehr als $10 \%$ in den letzten 6 Monaten vor der Operation und/oder Serumalbumin $<30 \mathrm{~g} / \mathrm{L}$, deutliche Kachexie) vor elektiver Operation präoperativ eine gezielte Ernährungstherapie für mindestens 7 Tage erfolgen. Expertenkonsens, Empfehlung, starker Konsens 


\section{Hintergrund}

Patienten mit schwerer Mangelernährung haben ein signifikant erhöhtes Risiko für postoperative Komplikationen [712, 732, 733]. Da in einer Studie ein positiver Effekt nur gesehen wurde [734], wenn die parenterale Ernährung mindestens 5 Tage vor der Operation begonnen wurde, sollte eine gezielte präoperative Ernährungstherapie vor elektiven Engriffen über mindestens 7 Tage durchgeführt werden. Zur Durchführung sei auf Kommentar 6.1.4. verwiesen. Durch den Einsatz von TPE als perioperative Maßnahme kann eine Verbesserung des Ernährungszustands und somit eine Verminderung postoperativer Komplikationen erzielt werden [735]. Nach Definition liegt beim Eintreten eines oder mehrerer der folgenden Kriterien eine schwere Mangelernährung vor:

- Gewichtsverlust $>10$ - $15 \%$ innerhalb von 6 Monaten

- $B M I<18,5 \mathrm{~kg} / \mathrm{m}^{2}$

- Serumalbumin <30 g/L (keine Einschränkung der Leber- und Nierenfunktion)

Bei gegebener Indikation zur präoperativen Ernährung sollte die enterale Zufuhr über Trink- und Sondennahrung vorgezogen werden. Die enterale Ernährung ist zur Vermeidung nosokomialer Infektionen möglichst prästationär durchzuführen. Die parenterale Applikation sollte Patienten mit hoher entzündlicher Aktivität im Kolon und intestinaler Unverträglichkeit vorbehalten bleiben [736- 738].

\section{EMPFEHLUNG 6.1.8}

CU Patienten mit High-Output-Jejuno- oder -lleostomie sollten engmaschig im Hinblick auf Wasser- und Elektrolytbilanz überwacht werden.

Expertenkonsens, Empfehlung, starker Konsensus

\section{Hintergrund}

Ziel der Ernährungstherapie bei lleostomie ist es, in Abhängigkeit vom Restdarm den Wasser- und Elektrolytverlust, aber auch eine chemische Reizung des Stomas durch Lebensmittel zu vermeiden. Erst nach etwa acht (ggf. auch bis zwölf) Wochen kommt es zur Stabilisierung der Stuhlbeschaffenheit, die jedoch flüssig bis breiig bleibt. In der Adaptationsphase (s. o.) wirkt sich der Einsatz von „stopfenden“ Lebensmitteln wie Kartoffeln, Reis, Haferflocken, Bananen positiv aus. Ggf. ist auch der Einsatz quellender Lebensmittel und flüssigkeitsbindender Präparate wie Pektine zur besseren Eindickung des Stuhls hilfreich.

Grundlage der Ernährungstherapie bei Ileostomie, Kostaufbau und Adaptationsphase ist eine ausreichende Flüssigkeits- und Kochsalzaufnahme. Für den lleostomieträger ist wichtig zu wissen, dass jede Nahrungsaufnahme zu einer Entleerung des Stomas führt.

Der Flüssigkeitsbedarf liegt bei ca. 3 Litern pro Tag. Als Richtwert für eine ausreichende Flüssigkeitsaufnahme gilt eine Urinmenge von mindestens 1 Liter pro Tag (regelmäßige Kontrollen des Urinvolumens). Eine Kochsalzaufnahme von $6-9 \mathrm{~g}$ pro Tag wird empfohlen (z. B. gesalzene Fleisch- und Gemüsebrühen). Der empfohlene Ballaststoffanteil liegt bei 25 - $30 \mathrm{~g}$ pro Tag [739].

\section{EMPFEHLUNG 6.1.9}

Patienten mit Pouch sollten regelmäßig auf das Vorliegen eines Vitamin-D-, - $\mathrm{B}_{12}$ - und Eisenmangels untersucht werden. Expertenkonsens, Empfehlung, starker Konsens

\section{Hintergrund}

Anämien infolge eines Eisen- und/oder Vitamin- $\mathrm{B}_{12}$-Mangels zählen zu den häufigsten extraintestinalen Manifestationen nach IAP [740, 741]. Ein Eisenmangel wird je nach Definition und Methode bei 20 - 56\% [703, 742] (Pouchitis 77\%) [700] der Patienten, ein $\mathrm{B}_{12}$-Mangel bei $25 \%$, ein Vitamin-D-Mangel $(<21 \mathrm{ng} / \mathrm{ml})$ bei $22 \%$ und eine Vitamin-D-Insuffizienz $(<31 \mathrm{ng} / \mathrm{ml})$ bei $70 \%$ beschrieben [743]. Ein Vitamin-E- und/oder -A-Mangel findet sich im Kontext einer Fettmalabsorption bei bis $5 \%$ der Patienten [744]. Bei länger anhaltender Steatorrhoe sollten daher Vitamin-A- und -E-Spiegel zumindest einmal bestimmt werden. Die Daten zum Folsäurestatus sind inkongruent und erlauben keine Empfehlung [740].

\subsection{Komplementäre Therapieverfahren}

\section{Präambel}

Es gibt keine allgemein akzeptierte Definition komplementärer und alternativer Therapieverfahren. Der Begriff komplementärmedizinische Verfahren beinhaltet, dass die angewendeten Behandlungsmethoden additiv, d.h. als Ergänzung zu konventionellen Standardtherapien, angewendet werden. Verfahren, die die konventionellen Standardtherapien ausschließen, werden als alternative Therapieverfahren bezeichnet. Unkonventionelle Therapien sind alle Verfahren, die als nicht anerkannt und/oder wissenschaftlich überprüft gelten.

Innerhalb der vorliegenden Leitlinie wird die Kategorisierung von Wieland et al. [745] zu Complementary and Alternative Medicine (CAM) für die Cochrane Collaboration zugrunde gelegt. Danach werden fünf Kategorien unterschieden: (1) Mind-BodyVerfahren, (2) ganzheitliche medizinische Systeme, (3) pflanzliche Heilverfahren, (4) manipulative und körperbezogene Verfahren und (5) Energiemedizin.

Die Bezeichnung „alternative Therapieverfahren“ spiegelt in der Regel nicht die übliche Anwendungssituation wider, da komplementärmedizinische/naturheilkundliche Therapien keinesfalls ein Selbstverständnis beinhalten, eine Alternative zur konventionellen Therapie darzustellen. Die angloamerikanische Literatur unterscheidet weniger in alternativ und komplementär und verwendet den gemeinsamen Terminus „complementary and alternative medicine“ (CAM), der sich inzwischen international weitgehend durchgesetzt hat. Neuere Literatur spricht bei kombinierter Anwendung von konventioneller und komplementärer Methoden auch von integrativer Medizin.

\section{EMPFEHLUNG 6.2.1}

Alternativtherapien anstelle einer evidenzgesicherten Therapie sollen abgelehnt werden.

Expertenkonsens, starke Empfehlung, starker Konsens 


\section{Hintergrund}

Da Alternativtherapien anstelle einer evidenzgesicherten Therapie benutzt werden, sind diese abzulehnen, da definitionsgemäß keine äquipotente Wirkung nachgewiesen ist (Phytotherapeutika und andere immunmodulierende Substanzen mit geprüfter Äquipotenz zu einer Standardtherapie sind somit keine alternativen Heilmittel (siehe unten)). Komplementäre Therapien, die als Ergänzung einer Standardtherapie angewendet werden, sollten in Absprache mit den behandelnden Ärzten erfolgen und können eine Standardtherapie unterstützen. Das große öffentliche Interesse an Alternativ- und Komplementärmedizin rechtfertigt eine weitere Evaluation dieser Methoden. Das schließt die folgenden Methoden ein: traditionelle Chinesische Medizin inkl. Akupunktur, anthroposophische Medizin, Aromatherapie, ayurvedische Medizin, Homöopathie, immunmodulative Therapien, manuelle Therapien (Osteopathie, Massagen etc.), Mind-Body-Medizin, Nahrungsergänzungsmittel, Naturheilkunde, Qi Gong, Reiki.

\section{EMPFEHLUNG 6.2.2}

Die Beurteilung naturheilkundlicher und komplementärmedizinischer Verfahren soll nach Kriterien einer evidenzbasierten Medizin erfolgen.

Expertenkonsens, starke Empfehlung, starker Konsens

\section{Hintergrund}

Methodik und Fragestellung bestimmen gemäß CONSORTKonsens die Bewertung einer Evidence-based-Medicine(EBM)Hierarchisierung. Dies ist bei Bewertung komplementärmedizinischer Literatur zu berücksichtigen. Einige komplementäre Therapieverfahren (sog. komplexe individuelle therapeutische Interventionen) beziehen sich auf das salutogenetische Potenzial des Patienten und bedürfen der nicht verblindeten Arzt-Patienten-Beziehung, da korrigierende Interaktionen bei Verblindung zwischen Arzt und Patient nicht möglich sind. Salutogene Therapieansätze sind meist durch Lern- und Regulationsprozesse gekennzeichnet, die einer analogen Beziehungsgestaltung des Lehrers zu seinen Schülern entsprechen und als dialogisch charakterisiert werden können. Ein Randomised-controlled-Trial(RCT)-Studiendesign ist daher nicht immer durchführbar; dies ist bei einer EBM-Hierarchisierung zu berücksichtigen. Ferner zeichnen sich komplementärund alternativmedizinische Verfahren meist durch komplexe Verfahrensweisen aus, die durch einfache Wirknachweise eines Einzelfaktors nicht zu belegen sind, sondern systemisch erfasst werden müssen. Dazu sind Outcomestudien im Kohortenvergleich die geeignete Evaluationsmethode und in ihren Ergebnissen meist einem RCT gleichwertig.

\section{EMPFEHLUNG 6.2.3}

Patienten sollen über die Anwendung komplementärer Heilmethoden befragt werden. Der behandelnde Arzt soll mit ihnen über ihre Gründe für die Anwendung komplementärmedizinischer Verfahren sprechen.

Expertenkonsens, starke Empfehlung, starker Konsens

\section{Hintergrund}

In zahlreichen Studien wurde beschrieben, dass mindestens die Hälfte (31\% bis $68 \%$ ) der Patienten mit CED komplementäre Heilmethoden anwendet [746-752]. Bei Kindern mit CED ist der Gebrauch von CAM nicht geringer als bei Erwachsenen.

In einer repräsentativen Studie für Deutschland zu dem Thema lag die Inanspruchnahme bei 52,9\% [752]. Die am häufigsten benutzten komplementären Methoden durch CED-Patienten sind Homöopathie, Phytotherapie, traditionelle chinesische Medizin einschließlich Akupunktur, Diäten, Vitamine und Nahrungsergänzungsmittel. Prädiktoren für den Einsatz komplementärmedizinischer Verfahren sind ein höherer Bildungsstand, eine Vollwerternährung sowie eine kumulative Kortisontabletteneinnahme von mehr als $10 \mathrm{~g}$ Gesamtmenge. Ein höherer Body Mass Index (BMI) war negativ mit dem Einsatz von CAM verbunden.

Drei von vier Patienten gaben dabei Erfahrung mit mehr als einem Verfahren an. Nur $25 \%$ der Patienten fühlten sich ausreichend über komplementäre Verfahren aufgeklärt. Bis zu 80 \% gaben aber Interesse am Einsatz von komplementären Verfahren in der Zukunft an.

30 - 70 \% der Patienten informieren ihre Ärzte/innen nicht über die Anwendung komplementärer Heilmethoden. Die Anwendung und das Verschweigen komplementärer Heilmethoden wird durch die konventionell behandelnden Ärzte unterschätzt. Als Gründe für die Anwendung komplementärmedizinischer Verfahren wurden in den Studien die Suche nach der optimalen Therapie, der Wunsch, ohne Kortison auszukommen, Nebenwirkungen der konventionellen Therapie, der Wunsch nach Stärkung der Eigenaktivität und der Eigenverantwortung, ein ganzheitlicher Therapieansatz sowie Unzufriedenheit mit der konventionellen Therapie und (relatives) Therapieversagen genannt. Bei Kindern mit Morbus Crohn korrelierte der Gebrauch von CAM mit der Zunahme von Fehlstunden in der Schule, Internetgebrauch und einem schlechteren Verlauf der Erkrankung.

\section{EMPFEHLUNG 6.2.4}

Aufgrund des hohen Anteils an Patienten, die komplementärmedizinische Therapien anwenden, sollten Ärzte sich über diese Verfahren informieren.

Expertenkonsens, Empfehlung, Konsens

\section{Hintergrund}

Ursache einer Anwendung von CAM durch Betroffene ist unter anderem eine Unzufriedenheit mit der konventionellen Therapie (s. o.). Viele Betroffene sehen sich durch die naturwissenschaftlich orientierte konventionelle Medizin nicht in ihrer komplexen mehrdimensionalen Persönlichkeit erfasst. Die Auffassungen von Arzt und Patient zum Krankheitsverständnis und dem Umgang mit der Erkrankung wie auch zum Selbstbild und Weltbild unterscheiden sich häufig. So kommt es dazu, dass außerhalb der ärztlichen Versorgung Beratung und Hilfe von CAM-Therapeuten gesucht werden (z. B. bei Heilpraktikern). Nicht selten verfahren die Betroffenen dabei „zweigleisig“: Der Facharzt therapiert konventionell, und der Patient sucht parallel nach weiteren Therapiemög- 
lichkeiten, ohne dass der Arzt Kenntnis von den zusätzlich angewandten CAM-Verfahren erhält, und der CAM-Therapeut entbehrt des speziellen medizinischen Sachverstands bei Colitis ulcerosa. 15 - 50 \% der CED-Patienten berichten ihren behandelnden Ärzten nicht vom Gebrauch der komplementären Therapie aus Angst vor herabsetzender Bewertung durch den Arzt. Andererseits fragen weniger als $20 \%$ der behandelnden spezialisierten CED-Ärzte den Patienten nach dem Gebrauch von CAM. Um Gefahren in diesem Kontext zu minimieren, sollte eine „Zweigleisigkeit" der Therapie durch den Betroffenen vermieden werden und eine enge Abstimmung der Therapieverfahren erfolgen. Dazu sind ausreichende Kenntnisse der komplementären Therapieverfahren beim primär behandelnden Arzt erforderlich. Nur eine kompetente und sachliche Beratung auch auf dem Gebiet von CAM durch den primär behandelnden Arzt kann diese Zweigleisigkeit für den Patienten vermeiden. Idealerweise ist ein integratives Konzept anzustreben, bei dem konventionelle und komplementäre Therapieverfahren zu einer „Best Practice“ verschmelzen (WHO-Definition von integrativer Medizin).

Mind-Body-Verfahren

\section{EMPFEHLUNG 6.2.5}

Achtsamkeitsbasierte Verfahren zur Stressreduktion können komplementär zur Verbesserung der Lebensqualität eingesetzt werden.

Evidenzgrad 2, Empfehlungsgrad 0, Konsens

\section{Hintergrund}

Ein systematischer Review zu komplementären und alternativen Verfahren zur Behandlung von chronisch-entzündlichen Darmerkrankungen [753] identifizierte einen RCT mit Ein-JahresFollow-up zur Lebensstilmodifikation [754, 755]. In einer prospektiven randomisiert-kontrollierten Studie im Wartegruppendesign konnte mit Lebensstilmodifikationsprogrammen (Bewegung, Ernährung, Selbsthilfestrategien, Stressmanagement) über 10 Wochen bei Patienten in Remission oder mit geringer Krankheitsaktivität eine durchschnittliche Verbesserung des IBDQ zum Therapieende um 20 Punkte in der Interventionsgruppe erreicht werden (eine individuelle Verbesserung von 16 Punkten wird als klinisch relevant beurteilt). Der psychische Summenscore im SF36 zeigte eine signifikante Verbesserung im Vergleich zur Wartekontrollgruppe. In der Katamnese nach 12 Monaten ließ sich kein signifikanter Gruppenunterschied mehr nachweisen.

Ein systematischer Review zu komplementären und alternativen Verfahren zur Behandlung von chronisch-entzündlichen Darmerkrankungen identifizierte zwei RCTs, die den Effekt von Achtsamkeit zur Behandlung von Colitis ulcerosa evaluierten [756, 757]. Eine Studie inkludierte 66 Patienten mit Colitis ulcerosa oder Morbus Crohn in Remission und verglich multikonvergente Therapie (Achtsamkeitsmeditation mit Aspekten kognitiver Verhaltenstherapie) in Kombination mit Standardversorgung mit Standardversorgung allein [756]. Eine weitere Studie inkludierte 55 Patienten mit Colitis ulcerosa in Remission und verglich achtsamkeitsbasierte Stressreduktion (MBSR) mit einer Aufmerksam- keitskontrollgruppe [757]. Es zeigten sich keine signifikanten Unterschiede hinsichtlich Krankheitsaktivität oder psychologischer Variablen. Lediglich für Patienten mit zusätzlichem Reizdarmsyndrom fanden sich Effekte auf die Lebensqualität sowie für Patienten, die innerhalb des Studienverlaufs einen Schub erfuhren, Effekte auf das C-reaktive Protein und Stress.

Neben achtsamkeitsbasierten Verfahren zeigen auch weitere Verfahren aus dem Bereich der Mind-Body-Verfahren Hinweise auf Wirksamkeit: Ein systematischer Review zu komplementären und alternativen Verfahren zur Behandlung von chronisch-entzündlichen Darmerkrankungen [753] identifizierte einen RCT, der den Effekt von Entspannungstrainings auf CED evaluierte [758]. 56 Patienten wurden entweder der Relaxationsgruppe oder der Standardbehandlung zugeteilt. Es zeigten sich signifikante Verbesserungen hinsichtlich Schmerz, Ängstlichkeit, Depressivität, Stimmung, Stress und Lebensqualität für die Patienten in der Relaxationsgruppe, nicht aber für die in der Standardbehandlungsgruppe. Die positiven Effekte auf Schmerzen werden von einer weiteren Studie gestützt, die die Wirkung eines Relaxationsverfahrens an 40 Colitis-ulcerosa-Patienten mit chronischem Schmerz evaluierte [759]. Die Studienqualität ist aber kaum einschätzbar, weswegen die Ergebnisse mit Vorsicht zu interpretieren sind.

Ein RCT untersuchte die Effekte eines Programms zur Atmung, Bewegung und Meditation (BBMW) im Vergleich zu einer Edukationsgruppe auf physiologische und psychologische Symptome bei 29 Patienten mit CED [760]. Die Studie kam zu dem Ergebnis, dass BBMW durchgeführt werden kann, da es zu positiven Prä-post-Effekten führt. Ein Edukationsseminar erzielt allerdings ähnliche Effekte. Weiterhin ist die Methodik der Studie fragwürdig, und der Anteil an Colitis-ulcerosa-Patienten betrug lediglich 9, weswegen die vorliegenden Ergebnisse nur mit Vorbehalt interpretiert werden sollten.

Körperliche Bewegung könnte einen positiven Effekt auf Colitis ulcerosa haben, die Evidenzlage ist jedoch gering. In einem prospektiven RCT wurde der Effekt von Bewegung auf CED evaluiert. Die teilnehmenden 30 Patienten befanden sich entweder in Remission oder zeigten eine milde Krankheitsaktivität [761]. Nach zehn Wochen moderatem Jogging dreimal wöchentlich zeigte sich ein signifikanter Gruppenunterschied hinsichtlich des sozialen Wohlbefindens. Weitere Gruppenunterschiede wurden nicht gefunden. Dieses Ergebnis sollte allerdings mit Vorsicht interpretiert werden, da es sich um eine Pilotstudie handelt, in der Colitis-ulcerosa- und Morbus-Crohn-Patienten gleichzeitig betrachtet wurden, die Stichprobe klein war und die Methodik fragwürdig ist. Es gab keine unerwünschten Nebenwirkungen. Ein systematischer Review schlussfolgert, dass Bewegung einen positiven Effekt hat, inkludierte aber hauptsächlich keine RCTs [762].

\section{EMPFEHLUNG 6.2.6}

Yoga kann komplementär zur Verbesserung der Lebensqualität eingesetzt werden.

Evidenzgrad 2, Empfehlungsgrad 0, Konsens 


\section{Hintergrund}

Eine randomisiert-kontrollierte Pilotstudie evaluierte den Effekt von Yoga im Vergleich zu Standardbehandlung an $60 \mathrm{~Pa}-$ tienten mit Colitis ulcerosa in Remission [763]. Zum Follow-up nach 2 Monaten zeigte sich ein signifikanter Gruppenunterschied hinsichtlich Ängstlichkeit sowie kolikartigen Bauchschmerzen zugunsten der Yogagruppe. Eine weitere randomisiert-kontrollierte Studie inkludierte 77 Patienten mit Colitis ulcerosa in Remission und verminderter Lebensqualität. Es zeigte sich ein positiver Effekt auf die Lebensqualität in Woche 12 und 24 sowie auf die Krankheitsaktivität in Woche 24 [764].

\section{Ganzheitliche Medizinsysteme}

\section{EMPFEHLUNG 6.2.7}

Akupunktur kann im leichten bis moderaten Schub komplementär in der Therapie eingesetzt werden.

Evidenzgrad 3, Empfehlungsgrad 0, Konsens

\section{Hintergrund}

Zwei systematische Reviews zu komplementären und alternativen Verfahren bzw. Akupunktur zur Behandlung von chronischentzündlichen Darmerkrankungen identifizierten einen RCT, der Akupunktur und Moxibustion (10 Sitzungen in 5 Wochen) bei leichter bis moderat aktiver Colitis ulcerosa im Vergleich zu oberflächlicher Nadelung an Nicht-Akupunkturpunkten untersucht [753, 765]. Der CAI nahm nach der Akupunkturbehandlung gegenüber der Kontrollgruppe signifikant ab. Die sekundären Endpunkte Verbesserung der Lebensqualität und Verbesserung des Allgemeinzustands zeigten keine signifikanten Gruppenunterschiede zum Ende der Therapie [766].

Zwei Metaanalysen, die auch chinesische Studien mit einschlossen, schlussfolgerten, dass die methodische Qualität der vorhandenen Studien zu gering ist, um Schlussfolgerungen daraus zu ziehen [767, 768].

\section{Pflanzliche Heilverfahren}

\section{EMPFEHLUNG 6.2.8}

Plantago ovata kann komplementär in der remissionserhaltenden Behandlung eingesetzt werden.

Evidenzgrad 2, Empfehlungsgrad 0, Konsens

\section{Hintergrund}

102 Patienten mit Colitis ulcerosa in Remission wurden drei Gruppen zugeordnet und erhielten entweder Flohsamen, Flohsamen in Kombination mit Mesalazin oder Mesalazin allein. Nach 12 Monaten zeigten sich keine Unterschiede zwischen der Behandlung mit Mesalazin und der mit Flohsamen. Lediglich die Butyratkonzentration im Stuhl von Patienten, die Flohsamen erhalten hatten, war besser. Es gab keine ernsthaften unerwünschten Nebenwirkungen [769]. Es gibt in Deutschland zugelassene Arzneimittel mit Plantago ovata.

\section{STATEMENT 6.2.9}

Für die Therapie mit Curcumin komplementär zu einem Aminosalizylat liegen Studien mit positiven Ergebnissen in der Remissionsinduktion sowie in der Remissionserhaltung vor. Curcumin steht in Deutschland nicht als Arzneimittel zur Verfügung.

Evidenzgrad 2, Empfehlungsgrad 0, mehrheitliche Zustimmung

\section{Hintergrund}

Ein systematischer Review zu komplementären und alternativen Verfahren zur Behandlung von chronisch-entzündlichen Darmerkrankungen identifizierte zwei RCTs, die die Effekte von Curcumin zur Behandlung von Colitis ulcerosa evaluierten. In einer prospektiven, randomisierten, doppelblinden, plazebokontrollierten Multicenterstudie wurde $2 \times 1 \mathrm{~g} / \mathrm{d}$ Curcumin komplementär zu Sulfasalazin oder Mesalazin in der remissionserhaltenden Therapie bei Colitis ulcerosa über einen Zeitraum von 6 Monaten untersucht (22 Patienten in der Verumgruppe) [770]. Es zeigte sich ein signifikanter Gruppenunterschied im Hinblick auf Rezidivhäufigkeit, CAI und einen endoskopischen Index zugunsten der Verumgruppe zum Therapieende. Diese positiven Ergebnisse stützen zwei weitere qualitativ hochwertige RCTs [771, 772]: Curcumin ist überlegen in der Therapie der Colitis ulcerosa. Diese Effekte zeigten sich innerhalb einer Studie allerdings nur für die Per-Protocol- und nicht für die Intention-to-treat-Analyse [771]. Es zeigten sich keine Unterschiede im Hinblick auf die Nebenwirkungen. Curcumin wird in Deutschland nur als Nahrungsergänzungsmittel angeboten und liegt nicht als Arzneimittel vor.

\section{EMPFEHLUNG 6.2.10}

Eine Kombination aus Myrrhe, Kamilleblütenextrakt und Kaffeekohle kann komplementär in der remissionserhaltenden Behandlung eingesetzt werden.

Evidenzgrad 2, Empfehlungsgrad 0, Konsens

\section{Hintergrund}

Ein systematischer Review zu komplementären und alternativen Verfahren zur Behandlung von chronisch-entzündlichen Darmerkrankungen identifizierte eine qualitativ hochwertige klinische Studie zur Wirksamkeit und Sicherheit von Myrrhe, Kamilleblütenextrakt und Kaffeekohle (Myrrhinil intest ${ }^{\circledR}$ ) in der remissionserhaltenden Therapie bei Colitis ulcerosa an 96 Patienten [773]. Die Studie ergab Hinweise darauf, dass die Therapie mit Myrrhe, Kamillenblütenextrakt und Kaffeekohle der Standardtherapie mit Mesalazin in der remissionserhaltenden Therapie nicht unterlegen und sehr gut verträglich ist. Eine Follow-up-Fragebogenstudie sowie eine große Kohortenstudie dazu bekräftigten diese Hinweise [774, 775].

Es gibt in Deutschland ein zugelassenes traditionelles Arzneimittel mit Myrrhe, Kamilleblütenextrakt und Kaffeekohle. 
EMPFEHLUNG 6.2.11

Weitere unkonventionelle Verfahren können aufgrund der unzureichenden Datenlage nicht empfohlen werden.

Expertenkonsens, Empfehlung offen, Konsens

\section{Hintergrund}

An 78 Patienten mit Colitis ulcerosa und moderater Krankheitsaktivität wurde der Effekt von Granatapfelextrakt in Kombination mit Standardtherapie im Vergleich zu Plazebo mit Standardtherapie evaluiert. Schlussfolgerungen sind, dass Granatapfelextrakt eingesetzt werden kann, da es positive Effekte auf die Krankheitsaktivität und einzelne Symptome hatte, allerdings nicht über einen Plazeboeffekt hinaus. Unerwünschte Nebenwirkungen waren mild und moderat und nicht unterschiedlich zwischen den Gruppen [776].

Ein systematischer Review [753] identifizierte zwei nicht randomisierte Studien, die die Wirkung von Boswellia serrata (Weihrauch-Präparat) im Vergleich zu Sulfasalazin evaluierten. Es zeigten sich für beide Gruppen positive Effekte unter anderem hinsichtlich der Histologie und Stuhlparametern ohne signifikante Gruppenunterschiede. Nebenwirkungen inkludierten Sodbrennen, Übelkeit, Appetitverlust und Schmerzen im Oberbauch [777, 778]. Boswellia serrata wird in Deutschland nur als Nahrungsergänzungsmittel angeboten und liegt nicht als Arzneimittel vor.

Ein systematischer Review [753] identifizierte zwei qualitativ hochwertige RCTs, die die Effekte von HMPL-004 mit Andrografis paniculata als Hauptbestandteil (Heilpflanze, auch als Kalmegh bezeichnet) an insgesamt 344 Patienten mit Colitis ulcerosa evaluierten. Innerhalb der einen Studie bekamen die Patienten entweder acht Wochen lang HMPL-004 oder Mesalazin [779]. Beide Präparate wirkten sich positiv auf die Krankheitsaktivität aus, die Nebenwirkungen waren allerdings in der Mesalazingruppe erhöht. Innerhalb der zweiten Studie wurden über einen Zeitraum von 8 Wochen zwei Dosierungen (1200 mg und $1800 \mathrm{mg}$ ) von HMPL004 in Kombination mit Mesalamin und Plazebo in Kombination mit Mesalamin gegeben und miteinander verglichen [780]. Nach acht Wochen zeigte sich eine Überlegenheit der höheren Dosierung von HMPL-004 gegenüber Plazebo hinsichtlich klinischen Ansprechens auf die Medikation und Heilung der Schleimhaut, nicht jedoch hinsichtlich der klinischen Remission. HMPL-004 liegt in Deutschland nicht als Arzneimittel vor.

Ein RCT, der die Wirkung von Weizengrassaft im Vergleich zu Plazebosaft an 24 Patienten mit Colitis ulcerosa testete, zeigte, dass die Patienten der Experimentalgruppe nach vier Wochen eine signifikant geringere Krankheitsaktivität, weniger rektale Blutungen und weniger abdominelle Schmerzen hatten [781]. Es traten keine ernsthaften Nebenwirkungen auf.

Ein systematischer Review zu komplementären und alternativen Verfahren zur Behandlung von chronisch-entzündlichen Darmerkrankungen [753] identifizierte einen RCT, der die Wir- kung von Nachtkerzenöl an 43 Patienten mit Colitis ulcerosa im Vergleich zu Oliven- und Omega-3-Öl evaluierte [782]. Eine Überlegenheit für das Nachtkerzenöl zeigte sich lediglich hinsichtlich der Stuhlkonsistenz. Unerwünschte Nebenwirkungen wurden nicht erhoben.

In einer prospektiven, randomisierten, doppelblinden, plazebokontrollierten Studie wurden $100 \mathrm{ml}$ Aloe-vera-Gel über 4 Wochen (30 Patienten mit Verum, 14 Patienten mit Plazebo) bei leichter bis mäßiggradiger Colitis ulcerosa untersucht [783]. Aufgrund statistischer Mängel der Studie kann daraus keine Therapieempfehlung abgeleitet werden.

Ein RCT evaluierte den Effekt von Silymarin (Extrakt aus Mariendistelfrüchten) im Vergleich zu Plazebo an 80 Patienten [784]. Es zeigten sich keine signifikanten Gruppenunterschiede.

Ein weiterer RCT zur Wirksamkeit von Sophora (Extrakt des Japanischen Schnurbaums als Kapsel) im Vergleich zu Mesalazin an 126 Patienten mit Colitis ulcerosa [785] ergab Hinweise darauf, dass die Therapie mit Sophora der Standardtherapie mit Mesalazin nicht unterlegen sein könnte. Es traten keine relevanten Nebenwirkungen auf.

Eine offene Pilotstudie zeigt, dass die Einnahme eines Blaubeerpräparats, bestehend aus getrockneten Früchten und Saftkonzentrat, einen positiven Effekt auf die Krankheitsaktivität haben könnte [786]. Qualitativ hochwertige RCTs sind allerdings nötig, um dies zu bestätigen.

Zwei randomisierte, plazebokontrollierte klinische Studien an 141 Patienten mit aktiver Colitis ulcerosa zeigen, dass transdermales Nikotin in der Behandlung aktiver Colitis ulcerosa in Kombination mit Standardtherapie zusätzliche positive Effekte hat [787, 788]. Nicht effektiv scheint es hingegen in der remissionserhaltenden Therapie oder als alleinige Behandlungsmethode bei aktiver Colitis [789, 790]. Allerdings führt die Gabe von transdermalem Nikotin vor allem bei lebenslangen Nichtrauchern zu häufigen und teils schweren Nebenwirkungen.

Eine randomisiert-kontrollierte Pilotstudie verglich eine Sauerstofftherapie plus Standardmedikation mit Standardmedikation allein an Patienten mit aktiver Colitis ulcerosa [791]. Die Sauerstofftherapie zeigte keine signifikanten Effekte, allerdings war die Stichprobengröße auch zu gering.

Ein Review beinhaltete ein RCT zu Colitis ulcerosa [792]. In dieser prospektiven, randomisierten, doppelblinden, plazebokontrollierten monozentrischen Studie wurde eine Therapie mit 2500 Trichuris-suis-Eiern alle 2 Wochen über 12 Wochen bei aktiver Colitis ulcerosa (CAI > 4) untersucht [793]. Zum Therapieende zeigte sich ein signifikanter Gruppenunterschied im Hinblick auf den CAI zugunsten der Verumgruppe. Es zeigten sich keine schwerwiegenden Nebeneffekte. Das Präparat ist in Deutschland als Medikament nicht zugelassen. Da eine große randomisiert-kontrollierte Studie zur Remissionsinduktion bei Morbus Crohn negativ ausgefallen ist [794], wird der Therapieansatz derzeit nicht mehr verfolgt. Eine Empfehlung zur Therapie mit Trichuris suis ovata (TSO) ist nicht möglich. 


\begin{tabular}{|c|c|c|c|}
\hline \multicolumn{2}{|c|}{ ABKÜRZUNGEN } & FACED & $\begin{array}{l}\text { Fachangestellte für chronisch-entzündliche } \\
\text { Darmerkrankungen }\end{array}$ \\
\hline 5-ASA & 5-Aminosalizylate & FICE & \\
\hline ALGK & Arbeitsgemeinschaft gastroenterologischer & FMT & Fäkaler Mikrobiomtransfer \\
\hline & Klinikärzte e. V. & $\mathrm{GDH}$ & Glutamatdehydrogenase \\
\hline ALM & Adenomassoziierte Läsion oder Masse & $\mathrm{Hb}$ & Hämoglobin \\
\hline ASCA & Anti-Saccharomyces-cerevisiae-Antikörper & HBV & Hepatitis-B-Virus \\
\hline AWMF & Arbeitsgemeinschaft der Wissenschaftlichen & HDWLE & Hochauflösende Weißlicht-Endoskopie \\
\hline & Medizinischen Fachgesellschaften e. V. & HGIEN & Hochgradige intraepitheliale Neoplasie \\
\hline AZA & Azathioprin & HIV & Humanes Immunschwäche-Virus \\
\hline BMI & Body Mass Index & $\mathrm{HLH}$ & Haemophagozytische Lymphohistiozytose \\
\hline BSG & Blutkörperchensenkungsgeschwindigkeit & i. m. & Intramuskulär \\
\hline C. difficile & Clostridium difficile & IAP & Ileoanaler Pouch \\
\hline CAM & Complementary and alternative Medicine & IBD & Inflammatory Bowel Disease \\
\hline CDI & Clostridium-difficile-Infektion & IBDQ & Inflammatory Bowel Disease Questionnaire \\
\hline CEBM & Centre for Evidence-Based Medicine & IEN & Intraepitheliale Neoplasien \\
\hline CED & Chronisch-entzündliche Darmerkrankungen & IfSG & Infektionsschutzgesetz \\
\hline CEUS & Kontrastverstärkte Ultraschalluntersuchung & IFX & Infliximab \\
\hline CGS & Clinical Guideline Services & IGRA & Interferon-gamma-Release-Assay \\
\hline CMV & Cytomegalievirus & $\mathrm{INH}$ & Isoniazid \\
\hline CRP & C-reaktives Protein & IPAA & Ileoanale Pouchanlage \\
\hline CsA & Cyclosporin A & IPS & Irritables Pouchsyndrom \\
\hline CT & Computertomografie & KRK & Kolorektales Karzinom \\
\hline CU & Colitis ulcerosa & LTBI & Latente tuberkulöse Infektion \\
\hline DALM & Dysplasieassoziierte Läsion oder Masse & LGIEN & Leichtgradige intraepitheliale Neoplasie \\
\hline DCCV & Deutsche Morbus Crohn/Colitis ulcerosa & MAS & Makrophagen-Aktivierungssyndrom \\
\hline DEGAM & $\begin{array}{l}\text { Vereinigung } \\
\text { Deutsche Gesellschaft für Allgemeinmedizin }\end{array}$ & MBSR & $\begin{array}{l}\text { Achtsamkeitsbasierte Stressreduktion } \\
\text { (Mindfulness-Based Stress Reduction) }\end{array}$ \\
\hline & und Familienmedizin & MMA & Methyl-Malonsäure \\
\hline DGIM & Deutsche Gesellschaft für Innere Medizin & MMX & Multi-Matrix-System \\
\hline DELBI & Deutsches Instrument zur methodischen & MRT & Magnetresonanztomographie \\
\hline & Leitlinienbewertung & MTX & Methotrexat \\
\hline DGAV & Deutsche Gesellschaft für Allgemein- und & $\mathrm{n}$ & Anzahl \\
\hline & Viszeralchirurgie & $\mathrm{NBI}$ & Narrow Band Imaging \\
\hline DGCH & Deutsche Gesellschaft für Chirurgie & NRS & Nicht randomisierte Studien \\
\hline DGEM & Deutsche Gesellschaft für Ernährungsmedizin & NSAR & Nichtsteroidale Antirheumatika \\
\hline DGK & Deutsche Gesellschaft für Koloproktologie & PANCA & Perinukleäre anti-neutrophile cytoplasmatische \\
\hline DGP & Deutsche Gesellschaft für Pathologie & & Antikörper \\
\hline DGVS & Deutsche Gesellschaft für Gastroenterologie, & PCR & Polymerase-Kettenreaktion \\
\hline & Verdauungs- und Stoffwechselkrankheiten & PDAI & Perianal Disease Activity Index \\
\hline DPGE & Gesellschaft für pädiatrische Gastroenterologie & PEG & Perkutane endoskopische Gastrostomie \\
\hline & und Ernährungsmedizin & PJP & Pneumocystis-jirovecii-Pneumonie \\
\hline E. coli & Escherichia coli & PMN & Polymorphonuklear \\
\hline EBM & Evidence-based Medicine & PSC & Primär sklerosierende Cholangitis \\
\hline EBV & Ebstein-Barr-Virus & PTLB & Posttraumatische Belastungsstörung \\
\hline ECCO & European Crohn's and Colitis Organization & PUCAI & Pediatric Ulcerative Colitis Activity Index \\
\hline EcN & Escherichia coli Nissle & RDS & Reizdarmsyndrom \\
\hline EMA & European Medicines Agency & RLD & Raised Lesions with Dysplasia \\
\hline ESCMID & European Society of Clinical Microbiology and & s. C. & Subkutan \\
\hline & Infectious Diseases & SMX & Sulfamethoxazol \\
\hline ESPEN & Europäische Gesellschaft für Parenterale und & $\mathrm{TB}$ & Tuberkulose \\
\hline & Enterale Ernährung & THT & Tuberkulinhauttest \\
\hline ESPGHAN & European Society of Pediatric Gastroenterology, & TMP/SMX & Trimethoprim \\
\hline & Hepatology and Nutrition & TNF & Tumornekrosefaktor \\
\hline
\end{tabular}


UCEIS Ulcerative Colitis Endoscopic Index of Severity

VZV Varizella-Zoster-Virus

WHO World Health Organisation

WLE Hochauflösende Weißlichtendoskopie

\section{Interessenkonflikt}

Im Einklang mit dem AWMF-Regelwerk zum Umgang mit Interessenskonflikten haben alle Teilnehmer Erklärungen auf dem entsprechenden AWMF-Formular vor Beginn der Konsensuskonferenz abgegeben. Die Interessenkonflikte wurden von denKoordinatoren der Leitlinie und Frau Lynen gesichtet und der Leitliniengruppe vor Beginn der Konsensuskonferenz präsentiert. Die Mandatsträger der Leitlinie gaben eine Vielzahl von Interessenkonflikten an. Nach Einschätzung der Leitliniengruppe stellen dieinterdisziplinäre Besetzung der Leitliniengruppe (einschließlich stimmberechtigter Patientenvertreter) und die systematische, extern durchgeführte Literaturrecherche und Bewertung eine wichtige Maßnahme zum Ausgleich dieser Interessenkonflikte dar. Mandatsträger mit personenbezogenen Zuwendungen (Zugehörigkeit zu Advisory Boards, Gutachter- und Vortragstätigkeit) wurden daher nach kritischer Bewertung durch die Leitliniengruppe nicht von den Abstimmungen ausgeschlossen, wenn die Art der Zuwendungen nicht einseitig (z. B. Zugehörigkeit zu mehreren Advisory Boards) und die wissenschaftliche Expertise nicht verzichtbar war. Finanzielle Zuwendungen, die ausschließlich wissenschaftlichen Institutionen zugeordnet werden konnten, führten nicht zu einer Stimmenthaltung (Drittmittel, Studienbeteiligung). Mandatsträger, deren Interessenkonflikte nicht vorlagen, oder Mandatsträger mit Eigentümerinteressen (z. B. Patente, Aktienbesitz, Firmenzugehörigkeit) erhielten kein Stimmrecht. Nach Überprüfung aller Interessenkonflikte wurden keine Mandatsträger ausgeschlossen. Die Interessenkonflikte sind im Leitlinienreport veröffentlicht.

\section{Literatur}

[1] Marteau P, Probert CS, Lindgren S et al. Combined oral and enema treatment with Pentasa (mesalazine) is superior to oral therapy alone in patients with extensive mild/moderate active ulcerative colitis: a randomised, double blind, placebo controlled study. Gut 2005; 54: 960 - 965

[2] Silverberg MS, Satsangi J, Ahmad T et al. Toward an integrated clinical, molecular and serological classification of inflammatory bowel disease: Report of a Working Party of the 2005 Montreal World Congress of Gastroenterology. Can J Gastroenterol 2005; 19: 5-36

[3] Ekbom A, Helmick C, Zack M et al. Ulcerative colitis and colorectal cancer. A population-based study. N Engl J Med 1990; 323: 1228-1233

[4] Soetikno RM, Lin OS, Heidenreich PA et al. Increased risk of colorectal neoplasia in patients with primary sclerosing cholangitis and ulcerative colitis: a meta-analysis. Gastrointest Endosc 2002; 56: 48 - 54

[5] Forrest K, Symmons D, Foster P. Systematic review: is ingestion of paracetamol or non-steroidal anti-inflammatory drugs associated with exacerbations of inflammatory bowel disease? Aliment Pharmacol Ther 2004; 20: 1035-1043

[6] Takeuchi K, Smale S, Premchand P et al. Prevalence and mechanism of nonsteroidal anti-inflammatory drug-induced clinical relapse in patients with inflammatory bowel disease. Clin Gastroenterol Hepatol 2006; 4: $196-202$

[7] Sandborn W], Stenson WF, Brynskov J et al. Safety of celecoxib in patients with ulcerative colitis in remission: a randomized, placebocontrolled, pilot study. Clin Gastroenterol Hepatol 2006; 4: 203- 211
[8] Korzenik JR, Podolsky DK. Selective use of selective nonsteroidal antiinflammatory drugs in inflammatory bowel disease. Clin Gastroenterol Hepatol 2006; 4: 157-159

[9] Ho GT, Chiam P, Drummond $\mathrm{H}$ et al. The efficacy of corticosteroid therapy in inflammatory bowel disease: analysis of a 5-year UK inception cohort. Aliment Pharmacol Ther 2006; 24: 319-330

[10] Faubion WA Jr, Loftus EV Jr, Harmsen WS et al. The natural history of corticosteroid therapy for inflammatory bowel disease: a populationbased study. Gastroenterology 2001; 121: 255-260

[11] Toruner M, Loftus EV Jr, Harmsen WS et al. Risk factors for opportunistic infections in patients with inflammatory bowel disease. Gastroenterology 2008; 134: 929-936

[12] Mahid SS, Minor KS, Soto RE et al. Smoking and inflammatory bowel disease: a meta-analysis. Mayo Clin Proc 2006; 81: 1462-1471

[13] Hoie O, Wolters F, Riis L et al. Ulcerative colitis: patient characteristics may predict 10-yr disease recurrence in a European-wide populationbased cohort. Am J Gastroenterol 2007; 102: 1692 -1701

[14] Beaugerie L, Massot N, Carbonnel F et al. Impact of cessation of smoking on the course of ulcerative colitis. Am J Gastroenterol 2001; 96: 21132116

[15] Birrenbach T, Bocker U. Inflammatory bowel disease and smoking: a review of epidemiology, pathophysiology, and therapeutic implications. Inflamm Bowel Dis 2004; 10: 848-859

[16] Florin TH, Pandeya N, Radford-Smith GL. Epidemiology of appendicectomy in primary sclerosing cholangitis and ulcerative colitis: its influence on the clinical behaviour of these diseases. Gut 2004; 53: 973-979

[17] Vermeire S. Review article: genetic susceptibility and application of genetic testing in clinical management of inflammatory bowel disease. Aliment Pharmacol Ther 2006; 24 (Suppl. 3): 2-10

[18] Merrett MN, Mortensen N, Kettlewell M et al. Smoking may prevent pouchitis in patients with restorative proctocolectomy for ulcerative colitis. Gut 1996; 38: 362-364

[19] Joelsson M, Benoni C, Oresland T. Does smoking influence the risk of pouchitis following ileal pouch anal anastomosis for ulcerative colitis? Scand J Gastroenterol 2006; 41: 929-933

[20] Loftus EV Jr. Clinical epidemiology of inflammatory bowel disease: Incidence, prevalence, and environmental influences. Gastroenterology 2004; 126: $1504-1517$

[21] Rutgeerts P, D’Haens G, Hiele M et al. Appendectomy protects against ulcerative colitis. Gastroenterology 1994; 106: 1251-1253

[22] Frisch M, Johansen C, Mellemkjaer L et al. Appendectomy and subsequent risk of inflammatory bowel diseases. Surgery 2001; 130: 36-43

[23] Koutroubakis IE, Vlachonikolis IG, Kouroumalis EA. Role of appendicitis and appendectomy in the pathogenesis of ulcerative colitis: a critical review. Inflamm Bowel Dis 2002; 8: 277-286

[24] Cosnes J, Carbonnel F, Beaugerie L et al. Effects of appendicectomy on the course of ulcerative colitis. Gut 2002; 51: $803-807$

[25] Radford-Smith GL, Edwards JE, Purdie DM et al. Protective role of appendicectomy on onset and severity of ulcerative colitis and Crohn's disease. Gut 2002; 51: 808-813

[26] Childers RE, Eluri S, Vazquez C et al. Family history of inflammatory bowel disease among patients with ulcerative colitis: a systematic review and meta-analysis. J Crohns Colitis 2014; 8: 1480-1497

[27] Henriksen M, Jahnsen J, Lygren I et al. Are there any differences in phenotype or disease course between familial and sporadic cases of inflammatory bowel disease? Results of a population-based follow-up study. Am J Gastroenterol 2007; 102: 1955-1963

[28] IBD Working Group of the European Society for Paediatric Gastroenterology, Hepatology and Nutrition. Inflammatory bowel disease in children and adolescents: recommendations for diagnosis-the Porto criteria. J Pediatr Gastroenterol Nutr 2005; 41: 1-7 
[29] Langholz E, Munkholm P, Davidsen M et al. Course of ulcerative colitis: analysis of changes in disease activity over years. Gastroenterology 1994; 107: 3-11

[30] Henriksen M, Jahnsen J, Lygren I et al. Change of diagnosis during the first five years after onset of inflammatory bowel disease: results of a prospective follow-up study (the IBSEN Study). Scand J Gastroenterol 2006; 41: 1037 - 1043

[31] Price AB. Overlap in the spectrum of non-specific inflammatory bowel disease-'colitis indeterminate'. J Clin Pathol 1978; 31: 567 - 577

[32] Rodgers AD, Cummins AG. CRP correlates with clinical score in ulcerative colitis but not in Crohn's disease. Dig Dis Sci 2007; 52: 2063-2068

[33] Vermeire S, Van Assche G, Rutgeerts P. C-reactive protein as a marker for inflammatory bowel disease. Inflamm Bowel Dis 2004; 10: 661 - 665

[34] Prantera C, Davoli M, Lorenzetti R et al. Clinical and laboratory indicators of extent of ulcerative colitis. Serum C-reactive protein helps the most. J Clin Gastroenterol 1988; 10: 41 - 45

[35] Roseth AG, Aadland E, Grzyb K. Normalization of faecal calprotectin: a predictor of mucosal healing in patients with inflammatory bowel disease. Scand J Gastroenterol 2004; 39: 1017-1020

[36] Schoepfer AM, Beglinger C, Straumann A et al. Ulcerative colitis: Corre lation of the Rachmilewitz endoscopic activity index with fecal calprotectin, clinical activity, C-reactive protein, and blood leukocytes. Inflamm Bowel Dis 2009; 15: 1851 - 1858

[37] Mao R, Xiao YL, Gao X et al. Fecal calprotectin in predicting relapse of inflammatory bowel diseases: a meta-analysis of prospective studies. Inflamm Bowel Dis 2012; 18: 1894 - 1899

[38] Lin JF, Chen JM, Zuo JH et al. Meta-analysis: fecal calprotectin for assessment of inflammatory bowel disease activity. Inflamm Bowel Dis 2014; 20: $1407-1415$

[39] Korczowski B, Szybist W. Serum procalcitonin and C-reactive protein in children with diarrhoea of various aetiologies. Acta Paediatr 2004; 93: $169-173$

[40] Herrlinger KR, Dittmann R, Weitz G et al. Serum procalcitonin differentiates inflammatory bowel disease and self-limited colitis. Inflamm Bowel Dis 2004; 10: 229-233

[41] Mylonaki M, Langmead L, Pantes A et al. Enteric infection in relapse of inflammatory bowel disease: importance of microbiological examination of stool. Eur J Gastroenterol Hepatol 2004; 16: 775-778

[42] Brown W], Hudson MJ, Patrick S et al. Search for enteric microbial pathogens in patients with ulcerative colitis. Digestion 1992; 53: $121-128$

[43] Bou-Fakhredin R, Halawi R, Roumi J et al. Insights into the diagnosis and management of iron deficiency in inflammatory bowel disease. Expert Rev Hematol 2017; 10: $801-808$

[44] Stein J, Dignass AU. Management of iron deficiency anemia in inflammatory bowel disease - a practical approach. Ann Gastroenterol 2013; 26: $104-113$

[45] Cappellini MD, Comin-Colet J, de Francisco A et al. Iron deficiency across chronic inflammatory conditions: International expert opinion on definition, diagnosis, and management. Am J Hematol 2017; 92: 1068 1078

[46] Stein J, Hartmann F, Dignass AU. Diagnosis and management of iron deficiency anemia in patients with IBD. Nat Rev Gastroenterol Hepatol 2010; 7: 599-610

[47] Plevy S. Do serological markers and cytokines determine the indeterminate? J Clin Gastroenterol 2004; 38: S51 - S56

[48] Riis L, Vind I, Vermeire S et al. The prevalence of genetic and serologic markers in an unselected European population-based cohort of IBD patients. Inflamm Bowel Dis 2007; 13: 24-32

[49] Joossens S, Daperno M, Shums Z et al. Interassay and interobserver variability in the detection of anti-neutrophil cytoplasmic antibodies in patients with ulcerative colitis. Clin Chem 2004; 50: 1422-1425
[50] Reese GE, Constantinides VA, Simillis C et al. Diagnostic precision of antiSaccharomyces cerevisiae antibodies and perinuclear antineutrophil cytoplasmic antibodies in inflammatory bowel disease. Am J Gastroenterol 2006; 101: $2410-2422$

[51] Weber $P$, Koch M, Heizmann WR et al. Microbic superinfection in relapse of inflammatory bowel disease. J Clin Gastroenterol 1992; 14: 302 - 308

[52] Issa M, Vijayapal A, Graham MB et al. Impact of Clostridium difficile on inflammatory bowel disease. Clin Gastroenterol Hepatol 2007; 5: $345-351$

[53] Rodemann JF, Dubberke ER, Reske KA et al. Incidence of Clostridium difficile infection in inflammatory bowel disease. Clin Gastroenterol Hepatol 2007; 5: $339-344$

[54] Johal SS, Hammond J, Solomon K et al. Clostridium difficile associated diarrhoea in hospitalised patients: onset in the community and hospital and role of flexible sigmoidoscopy. Gut 2004; 53: 673-677

[55] Minami M, Ohta M, Ohkura T et al. Cytomegalovirus infection in severe ulcerative colitis patients undergoing continuous intravenous cyclosporine treatment in Japan. World J Gastroenterol 2007; 13: 754-760

[56] Matsuoka K, Iwao Y, Mori T et al. Cytomegalovirus is frequently reactivated and disappears without antiviral agents in ulcerative colitis patients. Am J Gastroenterol 2007; 102: 331 - 337

[57] Dimitroulia E, Spanakis N, Konstantinidou AE et al. Frequent detection of cytomegalovirus in the intestine of patients with inflammatory bowel disease. Inflamm Bowel Dis 2006; 12: 879-884

[58] Hommes DW, Sterringa G, van Deventer S] et al. The pathogenicity of cytomegalovirus in inflammatory bowel disease: a systematic review and evidence-based recommendations for future research. Inflamm Bowel Dis 2004; 10: 245 - 250

[59] Poullis A, Foster R, Northfield TC et al. Review article: faecal markers in the assessment of activity in inflammatory bowel disease. Aliment Pharmacol Ther 2002; 16: 675-681

[60] Langhorst J, Elsenbruch S, Mueller T et al. Comparison of 4 neutrophilderived proteins in feces as indicators of disease activity in ulcerative colitis. Inflamm Bowel Dis 2005; 11: 1085 -1091

[61] Menees SB, Powell C, Kurlander J et al. A meta-analysis of the utility of C-reactive protein, erythrocyte sedimentation rate, fecal calprotectin, and fecal lactoferrin to exclude inflammatory bowel disease in adults with IBS. Am J Gastroenterol 2015; 110: 444 -454

[62] Mosli MH, Zou G, Garg SK et al. C-Reactive Protein, Fecal Calprotectin, and Stool Lactoferrin for Detection of Endoscopic Activity in Symptomatic Inflammatory Bowel Disease Patients: A Systematic Review and Meta-Analysis. Am J Gastroenterol 2015; 110: 802 - 819; quiz 820

[63] D'Inca R, Dal Pont E, Di Leo V et al. Can calprotectin predict relapse risk in inflammatory bowel disease? Am J Gastroenterol 2008; 103: 2007 2014

[64] Costa F, Mumolo MG, Bellini M et al. Role of faecal calprotectin as noninvasive marker of intestinal inflammation. Dig Liver Dis 2003; 35: 642 647

[65] van Rheenen PF, Van de Vijver E, Fidler V. Faecal calprotectin for screening of patients with suspected inflammatory bowel disease: diagnostic meta-analysis. BMJ 2010; 341: c3369

[66] Van de Vijver E, Schreuder AB, Cnossen WR et al. Safely ruling out inflammatory bowel disease in children and teenagers without referral for endoscopy. Arch Dis Child 2012; 97: 1014-1018

[67] Walker TR, Land ML, Kartashov A et al. Fecal lactoferrin is a sensitive and specific marker of disease activity in children and young adults with inflammatory bowel disease. J Pediatr Gastroenterol Nutr 2007; 44: 414 422

[68] Joishy M, Davies I, Ahmed M et al. Fecal calprotectin and lactoferrin as noninvasive markers of pediatric inflammatory bowel disease. J Pediatr Gastroenterol Nutr 2009; 48: 48-54 
[69] Costa F, Mumolo MG, Ceccarelli L et al. Calprotectin is a stronger predictive marker of relapse in ulcerative colitis than in Crohn's disease. Gut 2005; 54: $364-368$

[70] Walkiewicz D, Werlin SL, Fish D et al. Fecal calprotectin is useful in predicting disease relapse in pediatric inflammatory bowel disease. Inflamm Bowel Dis 2008; 14: 669-673

[71] Carbonnel F, Lavergne A, Lemann M et al. Colonoscopy of acute colitis. A safe and reliable tool for assessment of severity. Dig Dis Sci 1994; 39 : $1550-1557$

[72] Alemayehu G, Jarnerot G. Colonoscopy during an attack of severe ulcerative colitis is a safe procedure and of great value in clinical decision making. Am J Gastroenterol 1991; 86: 187-190

[73] Fefferman DS, Farrell RJ. Endoscopy in inflammatory bowel disease: indications, surveillance, and use in clinical practice. Clin Gastroenterol Hepatol 2005; 3: $11-24$

[74] Deutsch DE, Olson AD. Colonoscopy or sigmoidoscopy as the initial evaluation of pediatric patients with colitis: a survey of physician behavior and a cost analysis. J Pediatr Gastroenterol Nutr 1997; 25: 26-31

[75] Melmed GY, Elashoff R, Chen GC et al. Predicting a change in diagnosis from ulcerative colitis to Crohn's disease: a nested, case-control study. Clin Gastroenterol Hepatol 2007; 5: 602 -608; quiz 525

[76] Bryant RV, Burger DC, Delo J et al. Beyond endoscopic mucosal healing in UC: histological remission better predicts corticosteroid use and hospitalisation over 6 years of follow-up. Gut 2016; 65: $408-414$

[77] Neurath MF, Travis SP. Mucosal healing in inflammatory bowel diseases: a systematic review. Gut 2012; 61: 1619-1635

[78] Shah SC, Colombel JF, Sands BE et al. Mucosal Healing Is Associated With Improved Long-term Outcomes of Patients With Ulcerative Colitis: A Systematic Review and Meta-analysis. Clin Gastroenterol Hepatol 2016; 14: $1245-1255$ e8

[79] Reinink AR, Lee TC, Higgins PD. Endoscopic Mucosal Healing Predicts Favorable Clinical Outcomes in Inflammatory Bowel Disease: A Metaanalysis. Inflamm Bowel Dis 2016; 22: 1859-1869

[80] Gisbert JP, Marin AC, Chaparro M. The Risk of Relapse after Anti-TNF Discontinuation in Inflammatory Bowel Disease: Systematic Review and Meta-Analysis. Am J Gastroenterol 2016; 111: 632-647

[81] Schroeder KW, Tremaine WJ, Ilstrup DM. Coated oral 5-aminosalicylic acid therapy for mildly to moderately active ulcerative colitis. A randomized study. N Engl J Med 1987; 317: 1625-1629

[82] Travis SP, Schnell D, Krzeski P et al. Developing an instrument to assess the endoscopic severity of ulcerative colitis: the Ulcerative Colitis Endoscopic Index of Severity (UCEIS). Gut 2012; 61: 535-542

[83] Truelove SC, Witts LJ. Cortisone in ulcerative colitis; final report on a therapeutic trial. Br Med J 1955; 2: 1041 - 1048

[84] Jakobovits SL, Travis SP. Management of acute severe colitis. Br Med Bull 2005; 75-76: $131-144$

[85] Turner D, Otley AR, Mack D et al. Development, validation, and evaluation of a pediatric ulcerative colitis activity index: a prospective multicenter study. Gastroenterology 2007; 133: 423 - 432

[86] Preiss JC, Bokemeyer B, Buhr HJ et al. Updated German clinical practice guideline on "Diagnosis and treatment of Crohn's disease" 2014. Z Gastroenterol 2014; 52: 1431 - 1484

[87] Parente F, Greco S, Molteni M et al. Role of early ultrasound in detecting inflammatory intestinal disorders and identifying their anatomical location within the bowel. Aliment Pharmacol Ther 2003; 18: 1009-1016

[88] Hollerbach S, Geissler A, Schiegl H et al. The accuracy of abdominal ultrasound in the assessment of bowel disorders. Scand J Gastroenterol 1998; 33: 1201 - 1208

[89] Maconi G, Ardizzone S, Parente F et al. Ultrasonography in the evaluation of extension, activity, and follow-up of ulcerative colitis. Scand J Gastroenterol 1999; 34: $1103-1107$
[90] Worlicek H, Lutz H, Heyder $\mathrm{N}$ et al. Ultrasound findings in Crohn's disease and ulcerative colitis: a prospective study. J Clin Ultrasound 1987; 15: $153-163$

[91] Bozkurt T, Richter F, Lux G. Ultrasonography as a primary diagnostic too in patients with inflammatory disease and tumors of the small intestine and large bowel. J Clin Ultrasound 1994; 22: 85-91

[92] Arienti V, Campieri M, Boriani L et al. Management of severe ulcerative colitis with the help of high resolution ultrasonography. Am J Gastroenterol 1996; 91: 2163-2169

[93] Faure C, Belarbi N, Mougenot JF et al. Ultrasonographic assessment of inflammatory bowel disease in children: comparison with ileocolonoscopy. J Pediatr 1997; 130: 147-151

[94] Pradel JA, David XR, Taourel P et al. Sonographic assessment of the normal and abnormal bowel wall in nondiverticular ileitis and colitis. Abdom Imaging 1997; 22: 167 - 172

[95] Haber HP, Busch A, Ziebach R et al. Ultrasonographic findings correspond to clinical, endoscopic, and histologic findings in inflammatory bowel disease and other enterocolitides. J Ultrasound Med 2002; 21: $375-382$

[96] Pascu M, Roznowski AB, Muller HP et al. Clinical relevance of transabdominal ultrasonography and magnetic resonance imaging in patients with inflammatory bowel disease of the terminal ileum and large bowel. Inflamm Bowel Dis 2004; 10: 373-382

[97] Parente F, Molteni M, Marino B et al. Are Colonoscopy and Bowel Ultrasound Useful for Assessing Response to Short-Term Therapy and Predicting Disease Outcome of Moderate-to-Severe Forms of Ulcerative Colitis?: A Prospective Study. Am J Gastroenterol 2010; 105: 1150 - 1157

[98] Ludwig D, Wiener S, Bruning A et al. Mesenteric blood flow is related to disease activity and risk of relapse in ulcerative colitis: a prospective follow up study. Gut 1999; 45: 546- 552

[99] Homann N, Klarmann U, Fellermann K et al. Mesenteric pulsatility index analysis predicts response to azathioprine in patients with Crohn's disease. Inflamm Bowel Dis 2005; 11: $126-132$

[100] Girlich C, Schacherer D, Jung EM et al. Comparison between quantitative assessment of bowel wall vascularization by contrast-enhanced ultrasound and results of histopathological scoring in ulcerative colitis. Int J Colorectal Dis 2012; 27: 193 - 198

[101] Rutter MD, Saunders BP, Wilkinson KH et al. Cancer surveillance in longstanding ulcerative colitis: endoscopic appearances help predict cancer risk. Gut 2004; 53: 1813-1816

[102] Gumaste V, Sachar DB, Greenstein AJ. Benign and malignant colorecta strictures in ulcerative colitis. Gut 1992; 33: $938-941$

[103] Reiser JR, Waye JD, Janowitz HD et al. Adenocarcinoma in strictures of ulcerative colitis without antecedent dysplasia by colonoscopy. Am J Gastroenterol 1994; 89: 119-122

[104] Andersen K, Vogt C, Blondin D et al. Multi-detector CT-colonography in inflammatory bowel disease: prospective analysis of CT-findings to high-resolution video colonoscopy. Eur J Radiol 2006; 58: 140 - 146

[105] Horsthuis K, Bipat S, Bennink RJ et al. Inflammatory bowel disease diagnosed with US, MR, scintigraphy, and CT: meta-analysis of prospective studies. Radiology 2008; 247: 64-79

[106] Fumery M, Pineton de Chambrun G, Stefanescu C et al. Detection of Dysplasia or Cancer in 3.5\% of Patients With Inflammatory Bowel Disease and Colonic Strictures. Clin Gastroenterol Hepatol 2015; 13: $1770-1775$

[107] Sawczenko A, Sandhu BK. Presenting features of inflammatory bowel disease in Great Britain and Ireland. Arch Dis Child 2003; 88: $995-$ 1000

[108] Ajaj WM, Lauenstein TC, Pelster G et al. Magnetic resonance colonography for the detection of inflammatory diseases of the large bowel: quantifying the inflammatory activity. Gut 2005; 54: 257-263 
[109] Levine A, Koletzko S, Turner D et al. ESPGHAN revised porto criteria for the diagnosis of inflammatory bowel disease in children and adolescents. J Pediatr Gastroenterol Nutr 2014; 58: 795-806

[110] Allison MC, Hamilton-Dutoit S], Dhillon AP et al. The value of rectal biopsy in distinguishing self-limited colitis from early inflammatory bowel disease. Q J Med 1987; 65: 985-995

[111] Bentley E, Jenkins D, Campbell F et al. How could pathologists improve the initial diagnosis of colitis? Evidence from an international workshop. J Clin Pathol 2002; 55: 955-960

[112] Dejaco C, Oesterreicher C, Angelberger S et al. Diagnosing colitis: a prospective study on essential parameters for reaching a diagnosis. Endoscopy 2003; 35: 1004-1008

[113] Dube AK, Cross SS, Lobo AJ. Audit of the histopathological diagnosis of non-neoplastic colorectal biopsies: achievable standards for the diagnosis of inflammatory bowel disease. J Clin Pathol 1998; 51: 378 - 381

[114] Dundas SA, Dutton J, Skipworth P. Reliability of rectal biopsy in distinguishing between chronic inflammatory bowel disease and acute selflimiting colitis. Histopathology 1997; 31: 60-66

[115] Jenkins D, Balsitis M, Gallivan S et al. Guidelines for the initial biopsy diagnosis of suspected chronic idiopathic inflammatory bowel disease. The British Society of Gastroenterology Initiative. J Clin Pathol 1997; 50: $93-105$

[116] Nostrant TT, Kumar NB, Appelman HD. Histopathology differentiates acute self-limited colitis from ulcerative colitis. Gastroenterology 1987; 92: 318-328

[117] Schumacher G, Kollberg B, Sandstedt B. A prospective study of first attacks of inflammatory bowel disease and infectious colitis. Histologic course during the 1st year after presentation. Scand J Gastroenterol 1994; 29: 318-332

[118] Seldenrijk CA, Morson BC, Meuwissen SG et al. Histopathological evaluation of colonic mucosal biopsy specimens in chronic inflammatory bowel disease: diagnostic implications. Gut 1991; 32: 1514-1520

[119] Surawicz CM, Belic L. Rectal biopsy helps to distinguish acute self-limited colitis from idiopathic inflammatory bowel disease. Gastroenterology 1984; 86: 104-113

[120] Tanaka M, Masuda T, Yao T et al. Observer variation of diagnoses based on simple biopsy criteria differentiating among Crohn's disease, ulcerative colitis, and other forms of colitis. J Gastroenterol Hepatol 2001; 16: $1368-1372$

[121] Tanaka M, Riddell RH, Saito $\mathrm{H}$ et al. Morphologic criteria applicable to biopsy specimens for effective distinction of inflammatory bowel disease from other forms of colitis and of Crohn's disease from ulcerative colitis. Scand J Gastroenterol 1999; 34: 55-67

[122] Tanaka M, Saito H, Fukuda S et al. Simple mucosal biopsy criteria differentiating among Crohn disease, ulcerative colitis, and other forms of colitis: measurement of validity. Scand J Gastroenterol 2000; 35: $281-286$

[123] Theodossi A, Spiegelhalter D], Jass ] et al. Observer variation and discriminatory value of biopsy features in inflammatory bowel disease. Gut 1994; 35: $961-968$

[124] McHugh JB, Appelman HD, McKenna BJ. The diagnostic value of endoscopic terminal ileum biopsies. Am J Gastroenterol 2007; 102: $1084-1089$

[125] Cherian S, Singh P. Is routine ileoscopy useful? An observational study of procedure times, diagnostic yield, and learning curve. Am J Gastroenterol 2004; 99: 2324-2329

[126] Geboes K, Ectors N, D’Haens G et al. Is ileoscopy with biopsy worthwhile in patients presenting with symptoms of inflammatory bowel disease? Am J Gastroenterol 1998; 93: 201 - 206

[127] Lemberg DA, Clarkson CM, Bohane TD et al. Role of esophagogastroduodenoscopy in the initial assessment of children with inflammatory bowel disease. J Gastroenterol Hepatol 2005; 20: 1696 - 1700
[128] Surawicz CM. Serial sectioning of a portion of a rectal biopsy detects more focal abnormalities: a prospective study of patients with inflammatory bowel disease. Dig Dis Sci 1982; 27: 434-436

[129] Surawicz CM, Meisel JL, Ylvisaker T et al. Rectal biopsy in the diagnosis of Crohn's disease: value of multiple biopsies and serial sectioning. Gastroenterology 1981; 80: 66-71

[130] Therkildsen MH, Jensen BN, Teglbjaerg PS et al. The final outcome of patients presenting with their first episode of acute diarrhoea and an inflamed rectal mucosa with preserved crypt architecture. A clinicopathologic study. Scand J Gastroenterol 1989; 24: 158-164

[131] Washington K, Greenson JK, Montgomery E et al. Histopathology of ulcerative colitis in initial rectal biopsy in children. Am J Surg Pathol 2002; 26: $1441-1449$

[132] Geboes K, Dalle I. Influence of treatment on morphological features of mucosal inflammation. Gut 2002; 50 (Suppl. 3): III37- III42

[133] Odze R, Antonioli D, Peppercorn M et al. Effect of topical 5-aminosalicylic acid (5-ASA) therapy on rectal mucosal biopsy morphology in chronic ulcerative colitis. Am J Surg Pathol 1993; 17: 869-875

[134] Kleer CG, Appelman HD. Ulcerative colitis: patterns of involvement in colorectal biopsies and changes with time. Am J Surg Pathol 1998; 22: 983-989

[135] North American Society for Pediatric Gastroenterology H, Nutrition, Colitis Foundation of A, et al. Differentiating ulcerative colitis from Crohn disease in children and young adults: report of a working group of the North American Society for Pediatric Gastroenterology, Hepatology, and Nutrition and the Crohn's and Colitis Foundation of America. J Pediatr Gastroenterol Nutr 2007; 44: 653-674

[136] Glickman JN, Bousvaros A, Farraye FA et al. Pediatric patients with untreated ulcerative colitis may present initially with unusual morphologic findings. Am J Surg Pathol 2004; 28: $190-197$

[137] Robert ME, Tang L, Hao LM et al. Patterns of inflammation in mucosal biopsies of ulcerative colitis: perceived differences in pediatric populations are limited to children younger than 10 years. Am J Surg Pathol 2004; 28: $183-189$

[138] Markowitz J, Kahn E, Grancher K et al. Atypical rectosigmoid histology in children with newly diagnosed ulcerative colitis. Am J Gastroenterol 1993; 88: 2034-2037

[139] Autschbach F, Hoffmann JCKA, Klump B eds Chronisch entzündliche Darmerkrankungen Das CED-Handbuch für Klinik und Praxis. Stuttgart: Thieme; 2004: 110-122

[140] Borchard F. Differential diagnosis of colitis. Verh Dtsch Ges Pathol 1999; 83: $110-121$

[141] Histopathologische Diagnostik chronisch entzündlicher Darmerkrankungen. Historischer Rückblick und aktuelle Übersicht. Pathologe 1999; 20: 276-287

[142] Gomes P, du Boulay C, Smith CL et al. Relationship between disease activity indices and colonoscopic findings in patients with colonic inflammatory bowel disease. Gut 1986; 27: 92 - 95

[143] D'Haens G, Van Deventer S, Van Hogezand R et al. Endoscopic and histological healing with infliximab anti-tumor necrosis factor antibodies in Crohn's disease: A European multicenter trial. Gastroenterology 1999; 116: 1029- 1034

[144] Riley SA, Mani V, Goodman MJ et al. Microscopic activity in ulcerative colitis: what does it mean? Gut 1991; 32: 174-178

[145] Bitton A, Peppercorn MA, Antonioli DA et al. Clinical, biological, and histologic parameters as predictors of relapse in ulcerative colitis. Gastroenterology 2001; 120: 13-20

[146] Nishio $Y$, Ando T, Maeda $O$ et al. Pit patterns in rectal mucosa assessed by magnifying colonoscope are predictive of relapse in patients with quiescent ulcerative colitis. Gut 2006; 55: $1768-1773$ 
[147] Marchal-Bressenot A, Salleron J, Boulagnon-Rombi C et al. Development and validation of the Nancy histological index for UC. Gut 2017; 66: $43-49$

[148] Mosli MH, Feagan BG, Zou G et al. Development and validation of a histological index for UC. Gut 2017; 66: 50 - 58

[149] Riddell RH, Goldman H, Ransohoff DF et al. Dysplasia in inflammatory bowel disease: standardized classification with provisional clinical applications. Hum Pathol 1983; 14: 931 - 968

[150] Goldman H. Significance and detection of dysplasia in chronic colitis. Cancer 1996; 78: $2261-2263$

[151] Pohl C, Hombach A, Kruis W. Chronic inflammatory bowel disease and cancer. Hepatogastroenterology 2000; 47: 57 - 70

[152] Melville DM, Jass JR, Morson BC et al. Observer study of the grading of dysplasia in ulcerative colitis: comparison with clinical outcome. Hum Pathol 1989; 20: 1008-1014

[153] Eaden J, Abrams K, McKay H et al. Inter-observer variation between general and specialist gastrointestinal pathologists when grading dysplasia in ulcerative colitis. J Pathol 2001; 194: 152-157

[154] Odze RD, Goldblum J, Noffsinger A et al. Interobserver variability in the diagnosis of ulcerative colitis-associated dysplasia by telepathology. Mod Pathol 2002; 15: 379-386

[155] Dignass A, Eliakim R, Magro F et al. Second European evidence-based consensus on the diagnosis and management of ulcerative colitis part 1: definitions and diagnosis. J Crohns Colitis 2012; 6: 965 - 990

[156] Magro F, Gionchetti P, Eliakim R et al. Third European Evidence-based Consensus on Diagnosis and Management of Ulcerative Colitis. Part 1: Definitions, Diagnosis, Extra-intestinal Manifestations, Pregnancy, Cancer Surveillance, Surgery, and Ileo-anal Pouch Disorders. J Crohns Colitis 2017; 11: 649-670

[157] Laine L, Kaltenbach T, Barkun A et al. SCENIC international consensus statement on surveillance and management of dysplasia in inflammatory bowel disease. Gastrointest Endosc 2015; 81: 489-501 e26

[158] Farraye FA, Odze RD, Eaden J et al. AGA medical position statement on the diagnosis and management of colorectal neoplasia in inflammatory bowel disease. Gastroenterology 2010; 138: 738-745

[159] Rutter MD, Saunders BP, Wilkinson KH et al. Most dysplasia in ulcerative colitis is visible at colonoscopy. Gastrointest Endosc 2004; 60: $334-339$

[160] Blackstone MO, Riddell RH, Rogers BH et al. Dysplasia-associated lesion or mass (DALM) detected by colonoscopy in long-standing ulcerative colitis: an indication for colectomy. Gastroenterology 1981; 80: $366-374$

[161] Lennard-Jones JE, Melville DM, Morson BC et al. Precancer and cancer in extensive ulcerative colitis: findings among 401 patients over 22 years. Gut 1990; 31: $800-806$

[162] Butt JH, Konishi F, Morson BC et al. Macroscopic lesions in dysplasia and carcinoma complicating ulcerative colitis. Dig Dis Sci 1983; 28 : $18-26$

[163] Jonsson B, Ahsgren L, Andersson LO et al. Colorectal cancer surveillance in patients with ulcerative colitis. Br J Surg 1994; 81: 689-691

[164] Nugent FW, Haggitt RC, Gilpin PA. Cancer surveillance in ulcerative colitis. Gastroenterology 1991; 100: 1241 - 1248

[165] Lofberg R, Brostrom O, Karlen P et al. Colonoscopic surveillance in long-standing total ulcerative colitis-a 15-year follow-up study. Gastroenterology 1990; 99: 1021 - 1031

[166] Eaden J, Abrams K, Ekbom A et al. Colorectal cancer prevention in ulcerative colitis: a case-control study. Aliment Pharmacol Ther 2000; 14: $145-153$

[167] Rosenstock E, Farmer RG, Petras R et al. Surveillance for colonic carcinoma in ulcerative colitis. Gastroenterology 1985; 89: 1342 - 1346
[168] Karlen P, Kornfeld D, Brostrom O et al. Is colonoscopic surveillance reducing colorectal cancer mortality in ulcerative colitis? A population based case control study. Gut 1998; 42: 711 - 714

[169] Lashner BA, Turner BC, Bostwick DG et al. Dysplasia and cancer complicating strictures in ulcerative colitis. Dig Dis Sci 1990; 35: 349- 352

[170] Choi PM, Nugent FW, Schoetz DJ Jr et al. Colonoscopic surveillance reduces mortality from colorectal cancer in ulcerative colitis. Gastroenterology 1993; 105: $418-424$

[171] Bye WA, Nguyen TM, Parker CE et al. Strategies for detecting colon cancer in patients with inflammatory bowel disease (Review). Cochrane Database Syst Rev 2017; 9: CD000279. doi: 10.1002/14651858. CD000279.pub4

[172] Winther KV, Jess T, Langholz E et al. Long-term risk of cancer in ulcerative colitis: a population-based cohort study from Copenhagen County. Clin Gastroenterol Hepatol 2004; 2: 1088-1095

[173] Lutgens MW, Vleggaar FP, Schipper ME et al. High frequency of early colorectal cancer in inflammatory bowel disease. Gut 2008; 57: 1246 1251

[174] Lutgens MW, Oldenburg B, Siersema PD et al. Colonoscopic surveillance improves survival after colorectal cancer diagnosis in inflammatory bowel disease. $\mathrm{Br}$ J Cancer 2009; 101: 1671 - 1675

[175] Eaden JA, Abrams KR, Mayberry JF. The risk of colorectal cancer in ulcerative colitis: a meta-analysis. Gut 2001; 48: 526-535

[176] Lakatos L, Mester G, Erdelyi Z et al. Risk factors for ulcerative colitisassociated colorectal cancer in a Hungarian cohort of patients with ulcerative colitis: results of a population-based study. Inflamm Bowel Dis 2006; 12: 205-211

[177] Soderlund S, Brandt L, Lapidus A et al. Decreasing time-trends of colorectal cancer in a large cohort of patients with inflammatory bowel disease. Gastroenterology 2009; 136: 1561 -1567; quiz 1818-1819

[178] Gupta RB, Harpaz N, Itzkowitz S et al. Histologic inflammation is a risk factor for progression to colorectal neoplasia in ulcerative colitis: a cohort study. Gastroenterology 2007; 133: 1099-1105; quiz 13401341

[179] Jess T, Loftus EV Jr, Velayos FS et al. Risk of intestinal cancer in inflammatory bowel disease: a population-based study from olmsted county, Minnesota. Gastroenterology 2006; 130: 1039-1046

[180] Rutter MD, Saunders BP, Wilkinson KH et al. Thirty-year analysis of a colonoscopic surveillance program for neoplasia in ulcerative colitis. Gastroenterology 2006; 130: 1030 - 1038

[181] Velayos FS, Loftus EV Jr, Jess T et al. Predictive and protective factors associated with colorectal cancer in ulcerative colitis: A case-control study. Gastroenterology 2006; 130: 1941 -1949

[182] Jess T, Rungoe C, Peyrin-Biroulet L. Risk of colorectal cancer in patients with ulcerative colitis: a meta-analysis of population-based cohort studies. Clin Gastroenterol Hepatol 2012; 10: 639-645

[183] Jess T, Horvath-Puho E, Fallingborg J et al. Cancer risk in inflammatory bowel disease according to patient phenotype and treatment: a Danish population-based cohort study. Am J Gastroenterol 2013; 108: $1869-1876$

[184] Choi CH, Rutter MD, Askari A et al. Forty-Year Analysis of Colonoscopic Surveillance Program for Neoplasia in Ulcerative Colitis: An Updated Overview. Am J Gastroenterol 2015; 110: 1022-1034

[185] Selinger CP, Andrews JM, Titman A et al. Long-term follow-up reveals low incidence of colorectal cancer, but frequent need for resection, among Australian patients with inflammatory bowel disease. Clin Gastroenterol Hepatol 2014; 12: 644-650

[186] Annese V, Daperno M, Rutter MD et al. European evidence based consensus for endoscopy in inflammatory bowel disease. J Crohns Colitis 2013; 7: $982-1018$

[187] Thomas-Gibson S, Rogers P, Cooper S et al. Judgement of the quality of bowel preparation at screening flexible sigmoidoscopy is associated 
with variability in adenoma detection rates. Endoscopy 2006; 38: $456-460$

[188] Toruner M, Harewood GC, Loftus EV Jr et al. Endoscopic factors in the diagnosis of colorectal dysplasia in chronic inflammatory bowel disease. Inflamm Bowel Dis 2005; 11: 428-434

[189] Van Assche G, Dignass A, Bokemeyer B et al. Second European evidence-based consensus on the diagnosis and management of ulcerative colitis part 3: special situations. J Crohns Colitis 2013; 7: 1-33

[190] Rutter M, Saunders B, Wilkinson K et al. Severity of inflammation is a risk factor for colorectal neoplasia in ulcerative colitis. Gastroenterology 2004; 126: $451-459$

[191] Claessen MM, Lutgens MW, van Buuren HR et al. More right-sided IBD-associated colorectal cancer in patients with primary sclerosing cholangitis. Inflamm Bowel Dis 2009; 15: 1331 - 1336

[192] Cairns SR, Scholefield JH, Steele RJ et al. Guidelines for colorectal cancer screening and surveillance in moderate and high risk groups (update from 2002). Gut 2010; 59: 666-689

[193] Bernstein CN, Shanahan F, Weinstein WM. Are we telling patients the truth about surveillance colonoscopy in ulcerative colitis? Lancet 1994; 343: $71-74$

[194] Befrits R, Ljung T, Jaramillo E et al. Low-grade dysplasia in extensive, long-standing inflammatory bowel disease: a follow-up study. Dis Colon Rectum 2002; 45: 615-620

[195] Leidenius M, Kellokumpu I, Husa A et al. Dysplasia and carcinoma in longstanding ulcerative colitis: an endoscopic and histological surveillance programme. Gut 1991; 32: 1521 - 1525

[196] Jess T, Loftus EV Jr, Velayos FS et al. Incidence and prognosis of colorectal dysplasia in inflammatory bowel disease: a population-based study from Olmsted County, Minnesota. Inflamm Bowel Dis 2006; 12: $669-676$

[197] Thomas T, Abrams KA, Robinson RJ et al. Meta-analysis: cancer risk of low-grade dysplasia in chronic ulcerative colitis. Aliment Pharmacol Ther 2007; 25: 657-668

[198] Lim CH, Dixon MF, Vail A et al. Ten year follow up of ulcerative colitis patients with and without low grade dysplasia. Gut 2003; 52: 1127 1132

[199] Heuschen UA, Hinz U, Allemeyer EH et al. Backwash ileitis is strongly associated with colorectal carcinoma in ulcerative colitis. Gastroenterology 2001; $120: 841-847$

[200] Hlavaty T, Huorka M, Koller T et al. Colorectal cancer screening in patients with ulcerative and Crohn's colitis with use of colonoscopy, chromoendoscopy and confocal endomicroscopy. Eur J Gastroenterol Hepatol 2011; 23: 680-689

[201] Kiesslich R, Fritsch J, Holtmann M et al. Methylene blue-aided chromoendoscopy for the detection of intraepithelial neoplasia and colon cancer in ulcerative colitis. Gastroenterology 2003; 124: 880-888

[202] Hurlstone DP, Sanders DS, Lobo AJ et al. Indigo carmine-assisted highmagnification chromoscopic colonoscopy for the detection and characterisation of intraepithelial neoplasia in ulcerative colitis: a prospective evaluation. Endoscopy 2005; 37: 1186-1192

[203] Kiesslich R, Goetz M, Lammersdorf K et al. Chromoscopy-guided endomicroscopy increases the diagnostic yield of intraepithelial neoplasia in ulcerative colitis. Gastroenterology 2007; 132: 874-882

[204] Subramanian V, Mannath J, Ragunath K et al. Meta-analysis: the diagnostic yield of chromoendoscopy for detecting dysplasia in patients with colonic inflammatory bowel disease. Aliment Pharmacol Ther 2011; 33: 304-312

[205] Wu L, Li P, Wu J et al. The diagnostic accuracy of chromoendoscopy for dysplasia in ulcerative colitis: meta-analysis of six randomized controlled trials. Colorectal Dis 2012; 14: 416-420
[206] Carballal S, Maisterra S, Lopez-Serrano A et al. Real-life chromoendoscopy for neoplasia detection and characterisation in long-standing IBD. Gut 2018; 67: 70-78

[207] lannone A, Ruospo M, Wong G et al. Chromoendoscopy for Surveillance in Ulcerative Colitis and Crohn's Disease: A Systematic Review of Randomized Trials. Clin Gastroenterol Hepatol 2017; 15: 1684 1697

[208] Gasia MF, Ghosh S, Panaccione R et al. Targeted Biopsies Identify Larger Proportions of Patients With Colonic Neoplasia Undergoing High-Definition Colonoscopy, Dye Chromoendoscopy, or Electronic Virtual Chromoendoscopy. Clin Gastroenterol Hepatol 2016; 14: 704 $712 \mathrm{e} 4$

[209] Watanabe T, Ajioka Y, Mitsuyama K et al. Comparison of Targeted vs Random Biopsies for Surveillance of Ulcerative Colitis-Associated Colorectal Cancer. Gastroenterology 2016; 151: 1122 - 1130

[210] Moussata D, Allez M, Cazals-Hatem D et al. Are random biopsies still useful for the detection of neoplasia in patients with IBD undergoing surveillance colonoscopy with chromoendoscopy? Gut 2018; 67: $616-624$

[211] Subramanian V, Ramappa V, Telakis E et al. Comparison of high definition with standard white light endoscopy for detection of dysplastic lesions during surveillance colonoscopy in patients with colonic inflammatory bowel disease. Inflamm Bowel Dis 2013; 19: 350-355

[212] lannone A, Ruospo M, Wong G et al. Chromoendoscopy for Surveillance in Ulcerative Colitis and Crohn's Disease: A Systematic Review of Randomized Trials. Clin Gastroenterol Hepatol 2017; 15: 1684-1697 e11

[213] Dekker E, van den Broek FJ, Reitsma JB et al. Narrow-band imaging compared with conventional colonoscopy for the detection of dysplasia in patients with longstanding ulcerative colitis. Endoscopy 2007; 39: $216-221$

[214] Ignjatovic A, East JE, Subramanian V et al. Narrow band imaging for detection of dysplasia in colitis: a randomized controlled trial. Am J Gastroenterol 2012; 107: 885-890

[215] Pellise M, Lopez-Ceron M, Rodriguez de Miguel C et al. Narrow-band imaging as an alternative to chromoendoscopy for the detection of dysplasia in long-standing inflammatory bowel disease: a prospective, randomized, crossover study. Gastrointest Endosc 2011; 74: 840 - 848

[216] Gunther U, Kusch D, Heller F et al. Surveillance colonoscopy in patients with inflammatory bowel disease: comparison of random biopsy vs. targeted biopsy protocols. Int J Colorectal Dis 2011; 26: 667-672

[217] van den Broek FJ, van Es JA, van Eeden S et al. Pilot study of probebased confocal laser endomicroscopy during colonoscopic surveillance of patients with longstanding ulcerative colitis. Endoscopy 2011; 43: $116-122$

[218] Bisschops R, Bessissow T, Joseph JA et al. Chromoendoscopy versus narrow band imaging in UC: a prospective randomised controlled trial. Gut 2018; 67: 1087 - 1094

[219] Leifeld L, Rogler G, Stallmach A et al. White-Light or Narrow-Band Imaging Colonoscopy in Surveillance of Ulcerative Colitis: A Prospective Multicenter Study. Clin Gastroenterol Hepatol 2015; 13: 1776 1781 e1

[220] Omata F, Ohde S, Deshpande GA et al. Image-enhanced, chromo, and cap-assisted colonoscopy for improving adenoma/neoplasia detection rate: a systematic review and meta-analysis. Scand J Gastroenterol 2014; 49: $222-237$

[221] Taylor BA, Pemberton JH, Carpenter HA et al. Dysplasia in chronic ulcerative colitis: implications for colonoscopic surveillance. Dis Colon Rectum 1992; 35: $950-956$

[222] Fumery M, Dulai PS, Gupta S et al. Incidence, Risk Factors, and Outcomes of Colorectal Cancer in Patients With Ulcerative Colitis With LowGrade Dysplasia: A Systematic Review and Meta-analysis. Clin Gastroenterol Hepatol 2017; 15: 665-674 e5 
[223] Ullman T, Croog V, Harpaz N et al. Progression of flat low-grade dysplasia to advanced neoplasia in patients with ulcerative colitis. Gastroenterology 2003; 125: 1311 -1319

[224] Rubin PH, Friedman S, Harpaz N et al. Colonoscopic polypectomy in chronic colitis: conservative management after endoscopic resection of dysplastic polyps. Gastroenterology 1999; 117: 1295-1300

[225] Vieth M, Behrens H, Stolte M. Sporadic adenoma in ulcerative colitis: endoscopic resection is an adequate treatment. Gut 2006; 55: 1151 1155

[226] Odze RD, Farraye FA, Hecht JL et al. Long-term follow-up after polypectomy treatment for adenoma-like dysplastic lesions in ulcerative colitis. Clin Gastroenterol Hepatol 2004; 2: 534 - 541

[227] Wanders LK, Dekker E, Pullens B et al. Cancer risk after resection of polypoid dysplasia in patients with longstanding ulcerative colitis: a meta-analysis. Clin Gastroenterol Hepatol 2014; 12: 756-764

[228] Pardi DS, Loftus EV Jr, Kremers WK et al. Ursodeoxycholic acid as a chemopreventive agent in patients with ulcerative colitis and primary sclerosing cholangitis. Gastroenterology 2003; 124: 889-893

[229] Tung BY, Emond M], Haggitt RC et al. Ursodiol use is associated with lower prevalence of colonic neoplasia in patients with ulcerative colitis and primary sclerosing cholangitis. Ann Intern Med 2001; 134: 89-95

[230] Lindor KD, Kowdley KV, Luketic VA et al. High-dose ursodeoxycholic acid for the treatment of primary sclerosing cholangitis. Hepatology 2009; 50: 808-814

[231] LeBlanc K, Mosli MH, Parker CE et al. The impact of biological interventions for ulcerative colitis on health-related quality of life. Cochrane Database Syst Rev 2015; 22: CD008655

[232] Ford AC, Achkar JP, Khan KJ et al. Efficacy of 5-aminosalicylates in ulcerative colitis: systematic review and meta-analysis. Am J Gastroenterol 2011; 106: $601-616$

[233] Timmer A, Patton PH, Chande $\mathrm{N}$ et al. Azathioprine and 6-mercaptopurine for maintenance of remission in ulcerative colitis. Cochrane Database Syst Rev 2016; 18: CD000478

[234] Colombel JF, Rutgeerts P, Reinisch W et al. Early mucosal healing with infliximab is associated with improved long-term clinical outcomes in ulcerative colitis. Gastroenterology 2011; 141: 1194-1201

[235] Marshall JK, Thabane M, Steinhart AH et al. Rectal 5-aminosalicylic acid for induction of remission in ulcerative colitis. Cochrane Database Syst Rev 2010; 20: CD004115

[236] Lie MR, Kanis SL, Hansen BE et al. Drug therapies for ulcerative proctitis: systematic review and meta-analysis. Inflamm Bowel Dis 2014; 20: $2157-2178$

[237] Cohen RD, Dalal SR. Systematic Review: Rectal Therapies for the Treatment of Distal Forms of Ulcerative Colitis. Inflamm Bowel Dis 2015; $21: 1719-1736$

[238] Romkens TE, Kampschreur MT, Drenth JP et al. High mucosal healing rates in 5-ASA-treated ulcerative colitis patients: results of a metaanalysis of clinical trials. Inflamm Bowel Dis 2012; 18: 2190 -2198

[239] Andus T, Kocjan A, Muser M et al. Clinical trial: a novel high-dose $1 \mathrm{~g}$ mesalamine suppository (Salofalk) once daily is as efficacious as a 500-mg suppository thrice daily in active ulcerative proctitis. Inflamm Bowel Dis 2010; 16: 1947 - 1956

[240] Lamet M. A multicenter, randomized study to evaluate the efficacy and safety of mesalamine suppositories $1 \mathrm{~g}$ at bedtime and $500 \mathrm{mg}$ Twice daily in patients with active mild-to-moderate ulcerative proctitis. Dig Dis Sci 2011; 56: 513-522

[241] Gionchetti P, Rizzello F, Venturi A et al. Comparison of oral with rectal mesalazine in the treatment of ulcerative proctitis. Dis Colon Rectum 1998; 41: $93-97$

[242] Marshall JK, Irvine EJ. Rectal corticosteroids versus alternative treatments in ulcerative colitis: a meta-analysis. Gut 1997; 40: 775 - 781
[243] Regueiro M, Loftus EV Jr, Steinhart AH et al. Medical management of left-sided ulcerative colitis and ulcerative proctitis: critical evaluation of therapeutic trials. Inflamm Bowel Dis 2006; 12: 979-994

[244] Mulder C], Fockens P, Meijer JW et al. Beclomethasone dipropionate ( $3 \mathrm{mg}$ ) versus 5 -aminosalicylic acid $(2 \mathrm{~g})$ versus the combination of both $(3 \mathrm{mg} / 2 \mathrm{~g})$ as retention enemas in active ulcerative proctitis. Eur ] Gastroenterol Hepatol 1996; 8: 549-553

[245] Sandborn W], Bosworth B, Zakko S et al. Budesonide foam induces remission in patients with mild to moderate ulcerative proctitis and ulcerative proctosigmoiditis. Gastroenterology 2015; 148: 740 - 750 e2

[246] Bosworth BP, Sandborn WJ, Rubin DT et al. Baseline Oral 5-ASA Use and Efficacy and Safety of Budesonide Foam in Patients with Ulcerative Proctitis and Ulcerative Proctosigmoiditis: Analysis of 2 Phase 3 Studies. Inflamm Bowel Dis 2016; 22: 1881 - 1886

[247] Leifeld L, Pfutzer R, Morgenstern ] et al. Mesalazine granules are superior to Eudragit-L-coated mesalazine tablets for induction of remission in distal ulcerative colitis - a pooled analysis. Aliment Pharmacol Ther 2011; 34: 1115-1122

[248] Benson A, Barrett T, Sparberg M et al. Efficacy and safety of tacrolimus in refractory ulcerative colitis and Crohn's disease: a single-center experience. Inflamm Bowel Dis 2008; 14: 7 - 12

[249] Lawrance IC, Baird A, Lightower D et al. Efficacy of Rectal Tacrolimus for Induction Therapy in Patients With Resistant Ulcerative Proctitis. Clin Gastroenterol Hepatol 2017; 15: 1248-1255

[250] Ford AC, Khan KJ, Achkar JP et al. Efficacy of oral vs. topical, or combined oral and topical 5-aminosalicylates, in Ulcerative Colitis: systematic review and meta-analysis. Am J Gastroenterol 2012; 107: 167 - 176; author reply 177

[251] Pimpo MT, Galletti B, Palumbo G et al. Mesalazine vanishing time from rectal mucosa following its topical administration. J Crohns Colitis 2010; 4: $102-105$

[252] Cortot A, Maetz D, Degoutte E et al. Mesalamine foam enema versus mesalamine liquid enema in active left-sided ulcerative colitis. Am J Gastroenterol 2008; 103: 3106-3114

[253] Wang Y, Parker CE, Bhanji T et al. Oral 5-aminosalicylic acid for induction of remission in ulcerative colitis. Cochrane Database Syst Rev 2016; 4: CD000543

[254] Feagan BG, Chande N, MacDonald JK. Are there any differences in the efficacy and safety of different formulations of Oral 5-ASA used for induction and maintenance of remission in ulcerative colitis? evidence from cochrane reviews. Inflamm Bowel Dis 2013; 19: 2031 - 2040

[255] Manguso F, Balzano A. Meta-analysis: the efficacy of rectal beclomethasone dipropionate vs. 5-aminosalicylic acid in mild to moderate distal ulcerative colitis. Aliment Pharmacol Ther 2007; 26: 21 - 29

[256] Severs M, Mangen M], Fidder HH et al. Clinical Predictors of Future Nonadherence in Inflammatory Bowel Disease. Inflamm Bowel Dis 2017; 23: $1568-1576$

[257] Flourie B, Hagege H, Tucat G et al. Randomised clinical trial: once- vs. twice-daily prolonged-release mesalazine for active ulcerative colitis. Aliment Pharmacol Ther 2013; 37: 767-775

[258] Feagan BG, MacDonald JK. Once daily oral mesalamine compared to conventional dosing for induction and maintenance of remission in ulcerative colitis: a systematic review and meta-analysis. Inflamm Bowel Dis 2012; 18: 1785-1794

[259] Orchard TR, van der Geest SA, Travis SP. Randomised clinical trial: early assessment after 2 weeks of high-dose mesalazine for moderately active ulcerative colitis - new light on a familiar question. Aliment Pharmacol Ther 2011; 33: 1028-1035

[260] Sherlock ME, Seow CH, Steinhart AH et al. Oral budesonide for induction of remission in ulcerative colitis. Cochrane Database Syst Rev 2010; 6: CD007698 
[261] Sandborn W], Travis S, Moro L et al. Once-daily budesonide MMX(R) extended-release tablets induce remission in patients with mild to moderate ulcerative colitis: results from the CORE I study. Gastroenterology 2012; 143: 1218-1226 e1-2

[262] Travis SP, Danese S, Kupcinskas L et al. Once-daily budesonide MMX in active, mild-to-moderate ulcerative colitis: results from the randomised CORE II study. Gut 2014; 63: 433-441

[263] Sandborn W], Danese S, D'Haens G et al. Induction of clinical and colonoscopic remission of mild-to-moderate ulcerative colitis with budesonide MMX $9 \mathrm{mg}$ : pooled analysis of two phase 3 studies. Aliment Pharmacol Ther 2015; 41: 409-418

[264] Sherlock ME, MacDonald JK, Griffiths AM et al. Oral budesonide for induction of remission in ulcerative colitis. Cochrane Database Syst Rev 2015; 26: CD007698

[265] Marshall JK, Thabane M, Steinhart AH et al. Rectal 5-aminosalicylic acid for maintenance of remission in ulcerative colitis. Cochrane Database Syst Rev 2012; 11: CD004118

[266] Moody GA, Eaden JA, Helyes Z et al. Oral or rectal administration of drugs in IBD? Aliment Pharmacol Ther 1997; 11: 999-1000

[267] Chaparro M, Gisbert JP. Maintenance therapy options for ulcerative colitis. Expert Opin Pharmacother 2016; 17: 1339-1349

[268] Losurdo G, Iannone A, Contaldo A et al. Escherichia coli Nissle 1917 in Ulcerative Colitis Treatment: Systematic Review and Meta-analysis. J Gastrointestin Liver Dis 2015; 24: 499-505

[269] Scaldaferri F, Gerardi V, Mangiola F et al. Role and mechanisms of action of Escherichia coli Nissle 1917 in the maintenance of remission in ulcerative colitis patients: An update. World J Gastroenterol 2016; 22: $5505-5511$

[270] d'Albasio G, Pacini F, Camarri E et al. Combined therapy with 5-aminosalicylic acid tablets and enemas for maintaining remission in ulcerative colitis: a randomized double-blind study. Am J Gastroenterol 1997; 92: $1143-1147$

[271] Yokoyama H, Takagi S, Kuriyama S et al. Effect of weekend 5-aminosalicylic acid (mesalazine) enema as maintenance therapy for ulcerative colitis: results from a randomized controlled study. Inflamm Bowel Dis 2007; 13 : $1115-1120$

[272] Piodi LP, Ulivieri FM, Cermesoni L et al. Long-term intermittent treatment with low-dose 5 -aminosalicylic enemas is efficacious for remission maintenance in ulcerative colitis. Scand J Gastroenterol 2004; 39: $154-157$

[273] Bokemeyer B, Hommes D, Gill I et al. Mesalazine in left-sided ulcerative colitis: efficacy analyses from the PODIUM trial on maintenance of remission and mucosal healing. J Crohns Colitis 2012; 6: 476-482

[274] Kamm MA, Lichtenstein GR, Sandborn W] et al. Randomised trial of once- or twice-daily MMX mesalazine for maintenance of remission in ulcerative colitis. Gut 2008; 57: 893-902

[275] Feagan BG, Macdonald JK. Oral 5-aminosalicylic acid for maintenance of remission in ulcerative colitis. Cochrane Database Syst Rev 2012; 10: CD000544

[276] Dignass AU, Bokemeyer B, Adamek $\mathrm{H}$ et al. Mesalamine once daily is more effective than twice daily in patients with quiescent ulcerative colitis. Clin Gastroenterol Hepatol 2009; 7: 762 -769

[277] Gordon GL, Zakko S, Murthy U et al. Once-daily Mesalamine Formulation for Maintenance of Remission in Ulcerative Colitis: A Randomized, Placebo-controlled Clinical Trial. J Clin Gastroenterol 2016; 50: 318 325

[278] Kruis W, Jonaitis L, Pokrotnieks J et al. Randomised clinical trial: a comparative dose-finding study of three arms of dual release mesalazine for maintaining remission in ulcerative colitis. Aliment Pharmacol Ther 2011; 33: 313-322

[279] Sandborn W], Korzenik J, Lashner B et al. Once-daily dosing of delayedrelease oral mesalamine (400-mg tablet) is as effective as twice-daily dosing for maintenance of remission of ulcerative colitis. Gastroenterology 2010; 138: 1286-1296, 1296 e1-3

[280] Heyman MB, Kierkus J, Spenard J et al. Efficacy and safety of mesalamine suppositories for treatment of ulcerative proctitis in children and adolescents. Inflamm Bowel Dis 2010; 16: 1931-1939

[281] Kobayashi K, Hirai F, Naganuma $M$ et al. A randomized clinical trial of mesalazine suppository: the usefulness and problems of central review of evaluations of colonic mucosal findings. J Crohns Colitis 2014; 8: $1444-1453$

[282] Watanabe M, Nishino $\mathrm{H}$, Sameshima $\mathrm{Y}$ et al. Randomised clinical trial: evaluation of the efficacy of mesalazine (mesalamine) suppositories in patients with ulcerative colitis and active rectal inflammation - a placebo-controlled study. Aliment Pharmacol Ther 2013; 38: 264-273

[283] Kruis W, Jonaitis L, Pokrotnieks J et al. Randomised clinical trial: a comparative dose-finding study of three arms of dual release mesalazine for maintaining remission in ulcerative colitis. Aliment Pharmacol Ther 2011; 33: 313-322

[284] Yokoyama H, Takagi S, Kuriyama S et al. Effect of weekend 5-aminosalicylic acid (mesalazine) enema as maintenance therapy for ulcerative colitis: results from a randomized controlled study. Inflamm Bowel Dis 2007; 13: 1115-1120

[285] Ardizzone S, Porro GB. How long is it advisable to prolong maintenance treatment of patients with ulcerative colitis? Inflamm Bowel Dis 2008; 14 (Suppl. 2): \$238-\$239

[286] Heap GA, So K, Weedon M et al. Clinical Features and HLA Association of 5-Aminosalicylate (5-ASA)-induced Nephrotoxicity in Inflammatory Bowel Disease. J Crohns Colitis 2016; 10: 149-158

[287] Zallot C, Billioud V, Frimat L et al. 5-Aminosalicylates and renal function monitoring in inflammatory bowel disease: a nationwide survey. J Crohns Colitis 2013; 7: 551 - 555

[288] Nikolaus S, Schreiber S, Siegmund B et al. Patient Education in a 14-month Randomised Trial Fails to Improve Adherence in Ulcerative Colitis: Influence of Demographic and Clinical Parameters on Non-adherence. J Crohns Colitis 2017; 11: 1052-1062

[289] Velayos FS, Terdiman JP, Walsh JM. Effect of 5-aminosalicylate use on colorectal cancer and dysplasia risk: a systematic review and metaanalysis of observational studies. Am J Gastroenterol 2005; 100: $1345-1353$

[290] Biancone L, Michetti P, Travis S et al. European evidence-based Consensus on the management of ulcerative colitis: Special situations. J Crohns Colitis 2008; 2: 63-92

[291] Rubin DT, Huo D, Kinnucan JA et al. Inflammation is an independent risk factor for colonic neoplasia in patients with ulcerative colitis: a case-control study. Clin Gastroenterol Hepatol 2013; 11: 1601-1608 e1-4

[292] Mathy C, Schneider K, Chen YY et al. Gross versus microscopic pancolitis and the occurrence of neoplasia in ulcerative colitis. Inflamm Bowel Dis 2003; 9: 351-355

[293] Choi CH, Ignjatovic-Wilson A, Askari A et al. Low-grade dysplasia in ulcerative colitis: risk factors for developing high-grade dysplasia or colorectal cancer. Am J Gastroenterol 2015; 110: 1461 - 1471; quiz 1472

[294] Lindberg BU, Broome U, Persson B. Proximal colorectal dysplasia or cancer in ulcerative colitis. The impact of primary sclerosing cholangitis and sulfasalazine: results from a 20-year surveillance study. Dis Colon Rectum 2001; 44: 77-85

[295] Itzkowitz SH, Harpaz N. Diagnosis and management of dysplasia in patients with inflammatory bowel diseases. Gastroenterology 2004; 126: $1634-1648$

[296] Lyakhovich A, Gasche C. Systematic review: molecular chemoprevention of colorectal malignancy by mesalazine. Aliment Pharmacol Ther 2010; 31: 202-209 
[297] Ullman T, Croog V, Harpaz N et al. Progression to colorectal neoplasia in ulcerative colitis: effect of mesalamine. Clin Gastroenterol Hepatol 2008; 6: 1225-1230; quiz 1177

[298] Bergeron VNLI, Vienne A, Seksik P et al. Azathioprine [AZA] is associated with less histological inflammation of the colon in inactive IBD. Gastroenterology 2010; 138: 693

[299] Lashner BA, Provencher KS, Seidner DL et al. The effect of folic acid supplementation on the risk for cancer or dysplasia in ulcerative colitis. Gastroenterology 1997; 112: 29-32

[300] Terdiman JP, Steinbuch M, Blumentals WA et al. 5-Aminosalicylic acid therapy and the risk of colorectal cancer among patients with inflammatory bowel disease. Inflamm Bowel Dis 2007; 13: 367 - 371

[301] Matula S, Croog V, Itzkowitz S et al. Chemoprevention of colorectal neoplasia in ulcerative colitis: the effect of 6-mercaptopurine. Clin Gastroenterol Hepatol 2005; 3: 1015-1021

[302] van Schaik FD, van Oijen MG, Smeets HM et al. Thiopurines prevent advanced colorectal neoplasia in patients with inflammatory bowel disease. Gut 2012; 61: 235-240

[303] Beaugerie L, Svrcek M, Seksik P et al. Risk of colorectal high-grade dysplasia and cancer in a prospective observational cohort of patients with inflammatory bowel disease. Gastroenterology 2013; 145: 166 175 e8

[304] Lindgren S, Lofberg R, Bergholm L et al. Effect of budesonide enema on remission and relapse rate in distal ulcerative colitis and proctitis. Scand J Gastroenterol 2002; 37: $705-710$

[305] Meyers S, Lerer PK, Feuer E] et al. Predicting the outcome of corticoid therapy for acute ulcerative colitis. Results of a prospective, randomized, double-blind clinical trial. J Clin Gastroenterol 1987; 9: 50-54

[306] Lichtenstein GR, Feagan BG, Cohen RD et al. Serious infection and mortality in patients with Crohn's disease: more than 5 years of followup in the TREAT registry. Am J Gastroenterol 2012; 107: 1409-1422

[307] Edwards FC, Truelove SC. The Course and Prognosis of Ulcerative Colitis. Gut 1963; 4: 299-315

[308] Van Assche G, Vermeire S, Rutgeerts P. Management of acute severe ulcerative colitis. Gut 2011; 60: 130-133

[309] Turner D, Walsh CM, Steinhart AH et al. Response to corticosteroids in severe ulcerative colitis: a systematic review of the literature and a meta-regression. Clin Gastroenterol Hepatol 2007; 5: $103-110$

[310] Lynch RW, Lowe D, Protheroe A et al. Outcomes of rescue therapy in acute severe ulcerative colitis: data from the United Kingdom inflammatory bowel disease audit. Aliment Pharmacol Ther 2013; 38: 935 945

[311] Baron JH, Connell AM, Kanaghinis TG et al. Out-patient treatment of ulcerative colitis. Comparison between three doses of oral prednisone. Br Med J 1962; 2: 441 - 443

[312] Lennard-Jones JE, Longmore AJ, Newell AC et al. An assessment of prednisone, salazopyrin, and topical hydrocortisone hemisuccinate used as out-patient treatment for ulcerative colitis. Gut 1960; 1: 217 222

[313] Truelove SC, Jewell DP. Intensive intravenous regimen for severe attacks of ulcerative colitis. Lancet 1974; 1: 1067-1070

[314] Truelove SC, Witts L]. Cortisone in ulcerative colitis; preliminary report on a therapeutic trial. Br Med J 1954; 2: 375-378

[315] Truelove SC, Witts L]. Cortisone and corticotrophin in ulcerative colitis. Br Med J 1959; 1: $387-394$

[316] Rosenberg W, Ireland A, Jewell DP. High-dose methylprednisolone in the treatment of active ulcerative colitis. J Clin Gastroenterol 1990; 12: $40-41$

[317] Bartels SA, Gardenbroek T], Bos L et al. Prolonged preoperative hospital stay is a risk factor for complications after emergency colectomy for severe colitis. Colorectal Dis 2013; 15: 1392-1398
[318] Bernstein CN, Ng SC, Lakatos PL et al. A review of mortality and surgery in ulcerative colitis: milestones of the seriousness of the disease. Inflamm Bowel Dis 2013; 19: 2001 - 2010

[319] Randall J, Singh B, Warren BF et al. Delayed surgery for acute severe colitis is associated with increased risk of postoperative complications. Br J Surg 2010; 97: 404-409

[320] Roberts SE, Williams JG, Yeates D et al. Mortality in patients with and without colectomy admitted to hospital for ulcerative colitis and Crohn's disease: record linkage studies. BMJ 2007; 335: 1033

[321] Dignass AU, Gasche C, Bettenworth D et al. European consensus on the diagnosis and management of iron deficiency and anaemia in inflammatory bowel diseases. J Crohns Colitis 2015; 9: 211-222

[322] Autenrieth DM, Baumgart DC. Toxic megacolon. Inflamm Bowel Dis 2012; 18: $584-591$

[323] Gan SI, Beck PL. A new look at toxic megacolon: an update and review of incidence, etiology, pathogenesis, and management. Am J Gastroenterol 2003; 98: $2363-2371$

[324] Kvasnovsky CL, Aujla U, Bjarnason I. Nonsteroidal anti-inflammatory drugs and exacerbations of inflammatory bowel disease. Scand J Gastroenterol 2015; 50: 255-263

[325] Long MD, Barnes EL, Herfarth $\mathrm{HH}$ et al. Narcotic use for inflammatory bowel disease and risk factors during hospitalization. Inflamm Bowel Dis 2012; 18: $869-876$

[326] Domenech E, Vega R, Ojanguren I et al. Cytomegalovirus infection in ulcerative colitis: a prospective, comparative study on prevalence and diagnostic strategy. Inflamm Bowel Dis 2008; 14: 1373-1379

[327] Kishore J, Ghoshal U, Ghoshal UC et al. Infection with cytomegalovirus in patients with inflammatory bowel disease: prevalence, clinical significance and outcome. J Med Microbiol 2004; 53: 1155-1160

[328] Lee HS, Park SH, Kim SH et al. Risk Factors and Clinical Outcomes Associated with Cytomegalovirus Colitis in Patients with Acute Severe Ulcerative Colitis. Inflamm Bowel Dis 2016; 22: 912 - 918

[329] Papadakis KA, Tung JK, Binder SW et al. Outcome of cytomegalovirus infections in patients with inflammatory bowel disease. Am J Gastroenterol 2001; 96: $2137-2142$

[330] Rahier JF, Magro F, Abreu C et al. Second European evidence-based consensus on the prevention, diagnosis and management of opportunistic infections in inflammatory bowel disease. J Crohns Colitis 2014; 8: $443-468$

[331] Siegmund B. Cytomegalovirus infection associated with inflammatory bowel disease. Lancet Gastroenterol Hepatol 2017; 2: 369-376

[332] Berg AM, Kelly CP, Farraye FA. Clostridium difficile infection in the inflammatory bowel disease patient. Inflamm Bowel Dis 2013; 19: 194 204

[333] Issa M, Ananthakrishnan AN, Binion DG. Clostridium difficile and inflammatory bowel disease. Inflamm Bowel Dis 2008; 14: 1432 - 1442

[334] Jen MH, Saxena S, Bottle A et al. Increased health burden associated with Clostridium difficile diarrhoea in patients with inflammatory bowel disease. Aliment Pharmacol Ther 2011; 33: 1322 - 1331

[335] Murthy SK, Steinhart AH, Tinmouth J et al. Impact of Clostridium difficile colitis on 5-year health outcomes in patients with ulcerative colitis. Aliment Pharmacol Ther 2012; 36: 1032 - 1039

[336] Negron ME, Barkema HW, Rioux K et al. Clostridium difficile infection worsens the prognosis of ulcerative colitis. Can J Gastroenterol Hepato 2014; $28: 373-380$

[337] Nguyen GC, Kaplan GG, Harris ML et al. A national survey of the prevalence and impact of Clostridium difficile infection among hospitalized inflammatory bowel disease patients. Am J Gastroenterol 2008; 103: $1443-1450$

[338] Hagel S, Epple HJ, Feurle GE et al. S2k-guideline gastrointestinal infectious diseases and Whipple's disease. Z Gastroenterol 2015; 53: 418-459 
[339] Ben-Horin S, Margalit M, Bossuyt P et al. Combination immunomodulator and antibiotic treatment in patients with inflammatory bowel disease and clostridium difficile infection. Clin Gastroenterol Hepatol 2009; 7: $981-987$

[340] Rahier JF, Ben-Horin S, Chowers Y et al. European evidence-based Consensus on the prevention, diagnosis and management of opportunistic infections in inflammatory bowel disease. J Crohns Colitis 2009; 3: 47-91

[341] Grainge M], West ], Card TR. Venous thromboembolism during active disease and remission in inflammatory bowel disease: a cohort study. Lancet 2010; 375: 657-663

[342] Harbord M, Annese V, Vavricka SR et al. The First European Evidencebased Consensus on Extra-intestinal Manifestations in Inflammatory Bowel Disease. J Crohns Colitis 2016; 10: 239-254

[343] Kappelman MD, Horvath-Puho E, Sandler RS et al. Thromboembolic risk among Danish children and adults with inflammatory bowel diseases: a population-based nationwide study. Gut 2011; 60: 937 943

[344] Nguyen GC, Bernstein CN, Bitton A et al. Consensus statements on the risk, prevention, and treatment of venous thromboembolism in inflammatory bowel disease: Canadian Association of Gastroenterology. Gastroenterology 2014; 146: 835-848 e6

[345] Gonzalez-Huix F, Fernandez-Banares F, Esteve-Comas M et al. Enteral versus parenteral nutrition as adjunct therapy in acute ulcerative colitis. Am J Gastroenterol 1993; 88: 227-232

[346] McIntyre PB, Powell-Tuck J, Wood SR et al. Controlled trial of bowel rest in the treatment of severe acute colitis. Gut 1986; 27: 481-485

[347] Turner D, Travis SP, Griffiths AM et al. Consensus for managing acute severe ulcerative colitis in children: a systematic review and joint statement from ECCO, ESPGHAN, and the Porto IBD Working Group of ESPGHAN. Am J Gastroenterol 2011; 106: 574-588

[348] Lindgren SC, Flood LM, Kilander AF et al. Early predictors of glucocorticosteroid treatment failure in severe and moderately severe attacks of ulcerative colitis. Eur J Gastroenterol Hepatol 1998; 10: 831 -835

[349] Llao J, Naves JE, Ruiz-Cerulla A et al. Intravenous corticosteroids in moderately active ulcerative colitis refractory to oral corticosteroids. J Crohns Colitis 2014; 8: 1523-1528

[350] Rutgeerts P, Sandborn W], Feagan BG et al. Infliximab for induction and maintenance therapy for ulcerative colitis. N Engl J Med 2005; 353 : $2462-2476$

[351] Sandborn W], van Assche G, Reinisch W et al. Adalimumab induces and maintains clinical remission in patients with moderate-to-severe ulcerative colitis. Gastroenterology 2012; 142: 257-265 e1-3

[352] Sandborn W], Feagan BG, Marano C et al. Subcutaneous golimumab induces clinical response and remission in patients with moderate-tosevere ulcerative colitis. Gastroenterology 2014; 146: 85 - 95; quiz e14-15

[353] Sandborn W], Feagan BG, Marano C et al. Subcutaneous golimumab maintains clinical response in patients with moderate-to-severe ulcerative colitis. Gastroenterology 2014; 146: 96 - 109 e1

[354] Lennard-Jones JE, Ritchie JK, Hilder W et al. Assessment of severity in colitis: a preliminary study. Gut 1975; 16: 579-584

[355] Travis SP, Farrant JM, Ricketts $C$ et al. Predicting outcome in severe ulcerative colitis. Gut 1996; 38: 905-910

[356] Benazzato L, D’Inca R, Grigoletto F et al. Prognosis of severe attacks in ulcerative colitis: effect of intensive medical treatment. Dig Liver Dis 2004; 36: 461-466

[357] Chew CN, Nolan D], Jewell DP. Small bowel gas in severe ulcerative colitis. Gut 1991; 32: 1535-1537

[358] Carbonnel F, Gargouri D, Lemann M et al. Predictive factors of outcome of intensive intravenous treatment for attacks of ulcerative colitis. Aliment Pharmacol Ther 2000; 14: 273-279
[359] Corte C, Fernandopulle N, Catuneanu AM et al. Association between the ulcerative colitis endoscopic index of severity (UCEIS) and outcomes in acute severe ulcerative colitis. J Crohns Colitis 2015; 9: $376-$ 381

[360] Ho GT, Mowat C, Goddard C] et al. Predicting the outcome of severe ulcerative colitis: development of a novel risk score to aid early selection of patients for second-line medical therapy or surgery. Aliment Pharmacol Ther 2004; 19: 1079-1087

[361] Kato K, Ohkusa T, Terao S et al. Adjunct antibiotic combination therapy for steroid-refractory or -dependent ulcerative colitis: an open-label multicentre study. Aliment Pharmacol Ther 2014; 39: 949-956

[362] Mantzaris G], Hatzis A, Kontogiannis P et al. Intravenous tobramycin and metronidazole as an adjunct to corticosteroids in acute, severe ulcerative colitis. Am J Gastroenterol 1994; 89: 43-46

[363] Lichtiger S, Present DH, Kornbluth A et al. Cyclosporine in severe ulcerative colitis refractory to steroid therapy. N Engl J Med 1994; 330: $1841-1845$

[364] D’Haens G, Lemmens L, Geboes K et al. Intravenous cyclosporine versus intravenous corticosteroids as single therapy for severe attacks of ulcerative colitis. Gastroenterology 2001; 120: 1323-1329

[365] Van Assche G, D’Haens G, Noman M et al. Randomized, double-blind comparison of $4 \mathrm{mg} / \mathrm{kg}$ versus $2 \mathrm{mg} / \mathrm{kg}$ intravenous cyclosporine in severe ulcerative colitis. Gastroenterology 2003; 125: 1025-1031

[366] Laharie D, Bourreille A, Branche J et al. Ciclosporin versus infliximab in patients with severe ulcerative colitis refractory to intravenous steroids: a parallel, open-label randomised controlled trial. Lancet 2012; 380: 1909-1915

[367] Williams JG, Alam MF, Alrubaiy L et al. Infliximab versus ciclosporin for steroid-resistant acute severe ulcerative colitis (CONSTRUCT): a mixed methods, open-label, pragmatic randomised trial. Lancet Gastroenterol Hepatol 2016; 1: 15-24

[368] Shibolet O, Regushevskaya E, Brezis M et al. Cyclosporine A for induction of remission in severe ulcerative colitis. Cochrane Database Syst Rev 2005; 25: CD004277

[369] Cohen RD, Stein R, Hanauer SB. Intravenous cyclosporin in ulcerative colitis: a five-year experience. Am J Gastroenterol 1999; 94: 1587 1592

[370] Moskovitz DN, Van Assche G, Maenhout B et al. Incidence of colectomy during long-term follow-up after cyclosporine-induced remission of severe ulcerative colitis. Clin Gastroenterol Hepatol 2006; 4: 760-765

[371] Bamba S, Tsujikawa T, Inatomi $O$ et al. Factors affecting the efficacy of cyclosporin A therapy for refractory ulcerative colitis. J Gastroenterol Hepatol 2010; 25: 494-498

[372] Walch A, Meshkat M, Vogelsang $\mathrm{H}$ et al. Long-term outcome in patients with ulcerative colitis treated with intravenous cyclosporine $A$ is determined by previous exposure to thiopurines. J Crohns Colitis 2010; 4: $398-404$

[373] Cheifetz AS, Stern J, Garud S et al. Cyclosporine is safe and effective in patients with severe ulcerative colitis. J Clin Gastroenterol 2011; 45: $107-112$

[374] Ogata $\mathrm{H}$, Matsui T, Nakamura $\mathrm{M}$ et al. A randomised dose finding study of oral tacrolimus (FK506) therapy in refractory ulcerative colitis. Gut 2006; 55: $1255-1262$

[375] Ogata H, Kato J, Hirai F et al. Double-blind, placebo-controlled trial of oral tacrolimus (FK506) in the management of hospitalized patients with steroid-refractory ulcerative colitis. Inflamm Bowel Dis 2012; 18 : $803-808$

[376] Komaki Y, Komaki F, Ido A et al. Efficacy and Safety of Tacrolimus Therapy for Active Ulcerative Colitis; A Systematic Review and Metaanalysis. J Crohns Colitis 2016; 10: 484-494

[377] Schmidt KJ, Herrlinger KR, Emmrich J et al. Short-term efficacy of tacrolimus in steroid-refractory ulcerative colitis - experience in 130 patients. Aliment Pharmacol Ther 2013; 37: 129-136 
[378] Yamamoto T, Shimoyama T, Umegae S et al. Tacrolimus vs. anti-tumour necrosis factor agents for moderately to severely active ulcerative colitis: a retrospective observational study. Aliment Pharmacol Ther 2016; 43: 705-716

[379] Jarnerot G, Hertervig E, Friis-Liby I et al. Infliximab as rescue therapy in severe to moderately severe ulcerative colitis: a randomized, placebocontrolled study. Gastroenterology 2005; 128: 1805-1811

[380] Gustavsson A, Jarnerot G, Hertervig E et al. Clinical trial: colectomy after rescue therapy in ulcerative colitis - 3-year follow-up of the Swedish-Danish controlled infliximab study. Aliment Pharmacol Ther 2010; 32: 984-989

[381] Sjoberg M, Magnuson A, Bjork J et al. Infliximab as rescue therapy in hospitalised patients with steroid-refractory acute ulcerative colitis: a long-term follow-up of 211 Swedish patients. Aliment Pharmacol Ther 2013; 38: $377-387$

[382] Monterubbianesi R, Aratari A, Armuzzi A et al. Infliximab three-dose induction regimen in severe corticosteroid-refractory ulcerative colitis: early and late outcome and predictors of colectomy. J Crohns Colitis 2014; 8: 852-858

[383] Mortensen C, Caspersen S, Christensen NL et al. Treatment of acute ulcerative colitis with infliximab, a retrospective study from three Danish hospitals. J Crohns Colitis 2011; 5: 28-33

[384] Brandse JF, Mathot RA, van der Kleij D et al. Pharmacokinetic Features and Presence of Antidrug Antibodies Associate With Response to Infliximab Induction Therapy in Patients With Moderate to Severe Ulcerative Colitis. Clin Gastroenterol Hepatol 2016; 14: 251-258 e1-2

[385] Brandse JF, van den Brink GR, Wildenberg ME et al. Loss of Infliximab Into Feces Is Associated With Lack of Response to Therapy in Patients With Severe Ulcerative Colitis. Gastroenterology 2015; 149: 350-355 e2

[386] Gibson DJ, Heetun ZS, Redmond CE et al. An accelerated infliximab induction regimen reduces the need for early colectomy in patients with acute severe ulcerative colitis. Clin Gastroenterol Hepatol 2015; 13: $330-335$ e1

[387] Armuzzi A, Pugliese D, Danese S et al. Long-term combination therapy with infliximab plus azathioprine predicts sustained steroid-free clinical benefit in steroid-dependent ulcerative colitis. Inflamm Bowel Dis 2014; $20: 1368-1374$

[388] Chang KH, Burke JP, Coffey JC. Infliximab versus cyclosporine as rescue therapy in acute severe steroid-refractory ulcerative colitis: a systematic review and meta-analysis. Int J Colorectal Dis 2013; 28: 287 293

[389] Lowenberg M, Duijvis NW, Ponsioen C et al. Length of hospital stay and associated hospital costs with infliximab versus cyclosporine in severe ulcerative colitis. Eur J Gastroenterol Hepatol 2014; 26: 1240-1246

[390] Hyde GM, Jewell DP, Kettlewell MG et al. Cyclosporin for severe ulcerative colitis does not increase the rate of perioperative complications. Dis Colon Rectum 2001; 44: 1436-1440

[391] Ferrante M, D'Hoore A, Vermeire $S$ et al. Corticosteroids but not infliximab increase short-term postoperative infectious complications in patients with ulcerative colitis. Inflamm Bowel Dis 2009; 15: 1062 1070

[392] Narula N, Fine M, Colombel JF et al. Systematic Review: Sequential Rescue Therapy in Severe Ulcerative Colitis: Do the Benefits Outweigh the Risks? Inflamm Bowel Dis 2015; 21: 1683-1694

[393] Hebden JM, Blackshaw PE, Perkins AC et al. Limited exposure of the healthy distal colon to orally-dosed formulation is further exaggerated in active left-sided ulcerative colitis. Aliment Pharmacol Ther 2000; 14: $155-161$

[394] Nel JA. Management of patients with AMS 800 urinary artificial sphincter. Nurs RSA 1991; 6: 28-32
[395] Sandborn W], Tremaine W], Schroeder KW et al. A placebo-controlled trial of cyclosporine enemas for mildly to moderately active left-sided ulcerative colitis. Gastroenterology 1994; 106: 1429-1435

[396] Scheppach W. Treatment of distal ulcerative colitis with short-chain fatty acid enemas. A placebo-controlled trial. German-Austrian SCFA Study Group. Dig Dis Sci 1996; 41: 2254-2259

[397] van Dieren JM, van Bodegraven AA, Kuipers EJ et al. Local application of tacrolimus in distal colitis: feasible and safe. Inflamm Bowel Dis 2009; 15: $193-198$

[398] Carbonnel F, Boruchowicz A, Duclos B et al. Intravenous cyclosporine in attacks of ulcerative colitis: short-term and long-term responses. Dig Dis Sci 1996; 41: 2471 - 2476

[399] Actis GC, Fadda M, David E et al. Colectomy rate in steroid-refractory colitis initially responsive to cyclosporin: a long-term retrospective cohort study. BMC Gastroenterol 2007; 7: 13

[400] Szanto K, Molnar T, Farkas K. New promising combo therapy in inflammatory bowel diseases refractory to anti-TNF agents: cyclosporine plus vedolizumab. J Crohns Colitis 2018; 12: 629

[401] Ardizzone S, Maconi G, Russo A et al. Randomised controlled trial of azathioprine and 5 -aminosalicylic acid for treatment of steroid dependent ulcerative colitis. Gut 2006; 55: 47-53

[402] Chebli LA, Chaves LD, Pimentel FF et al. Azathioprine maintains longterm steroid-free remission through 3 years in patients with steroiddependent ulcerative colitis. Inflamm Bowel Dis 2010; 16: 613-619

[403] Panaccione R, Ghosh S, Middleton S et al. Combination therapy with infliximab and azathioprine is superior to monotherapy with either agent in ulcerative colitis. Gastroenterology 2014; 146: 392 - 400 e3

[404] Feagan BG, Rutgeerts P, Sands BE et al. Vedolizumab as induction and maintenance therapy for ulcerative colitis. N Engl J Med 2013; 369: $699-710$

[405] Carbonnel F, Colombel JF, Filippi ] et al. Methotrexate Is Not Superior to Placebo for Inducing Steroid-Free Remission, but Induces Steroid-Free Clinical Remission in a Larger Proportion of Patients With Ulcerative Colitis. Gastroenterology 2016; 150: 380 - 388 e4

[406] Lawson MM, Thomas AG, Akobeng AK. Tumour necrosis factor alpha blocking agents for induction of remission in ulcerative colitis. Cochrane Database Syst Rev 2006; 19: CD005112

[407] Reinisch W, Sandborn W], Hommes DW et al. Adalimumab for induction of clinical remission in moderately to severely active ulcerative colitis: results of a randomised controlled trial. Gut 2011; 60: 780 - 787

[408] Reinisch W, Sandborn W], Panaccione R et al. 52-week efficacy of adalimumab in patients with moderately to severely active ulcerative colitis who failed corticosteroids and/or immunosuppressants. Inflamm Bowel Dis 2013; 19: 1700-1709

[409] Gies N, Kroeker KI, Wong K et al. Treatment of ulcerative colitis with adalimumab or infliximab: long-term follow-up of a single-centre cohort. Aliment Pharmacol Ther 2010; 32: 522 - 528

[410] Colombel JF, Sandborn W], Reinisch W et al. Infliximab, azathioprine, or combination therapy for Crohn's disease. N Engl J Med 2010; 362: $1383-1395$

[411] van Schaik T, Maljaars JP, Roopram RK et al. Influence of combination therapy with immune modulators on anti-TNF trough levels and antibodies in patients with IBD. Inflamm Bowel Dis 2014; 20: 2292-2298

[412] Yamamoto S, Nakase H, Mikami S et al. Long-term effect of tacrolimus therapy in patients with refractory ulcerative colitis. Aliment Pharmacol Ther 2008; 28: 589-597

[413] Gisbert JP, Marin AC, McNicholl AG et al. Systematic review with metaanalysis: the efficacy of a second anti-TNF in patients with inflammatory bowel disease whose previous anti-TNF treatment has failed. Aliment Pharmacol Ther 2015; 41: 613-623

[414] Baumgart DC, Bokemeyer B, Drabik A et al. Vedolizumab induction therapy for inflammatory bowel disease in clinical practice-a nation- 
wide consecutive German cohort study. Aliment Pharmacol Ther 2016; 43: 1090 - 1102

[415] Sandborn WS, D'Haens G. Efficacy and safety of oral tofacitinib as induction therapy in patients with moderate to severe ulcerative colitis: results from two phase 3 randomized controlled trials. Gastroenterology 2016: S157

[416] Sandborn W], Feagan BG, Wolf DC et al. Ozanimod Induction and Maintenance Treatment for Ulcerative Colitis. N Engl J Med 2016; 374: $1754-1762$

[417] Yoo DH, Hrycaj P, Miranda P et al. A randomised, double-blind, parallel-group study to demonstrate equivalence in efficacy and safety of CT-P13 compared with innovator infliximab when coadministered with methotrexate in patients with active rheumatoid arthritis: the PLANETRA study. Ann Rheum Dis 2013; 72: 1613-1620

[418] Park W, Hrycaj P, Jeka S et al. A randomised, double-blind, multicentre, parallel-group, prospective study comparing the pharmacokinetics, safety, and efficacy of CT-P13 and innovator infliximab in patients with ankylosing spondylitis: the PLANETAS study. Ann Rheum Dis 2013; 72: $1605-1612$

[419] Jorgensen KK, Olsen IC, Goll GL et al. Switching from originator infliximab to biosimilar CT-P13 compared with maintained treatment with originator infliximab (NOR-SWITCH): a 52-week, randomised, doubleblind, non-inferiority trial. Lancet 2017; 389: 2304-2316

[420] Hawthorne AB, Logan RF, Hawkey C] et al. Randomised controlled trial of azathioprine withdrawal in ulcerative colitis. BMJ 1992; 305: 20-22

[421] Jewell DP, Truelove SC. Azathioprine in ulcerative colitis: final report on controlled therapeutic trial. Br Med J 1974; 4: 627-630

[422] Sood A, Kaushal V, Midha V et al. The beneficial effect of azathioprine on maintenance of remission in severe ulcerative colitis. J Gastroenterol 2002: $37: 270-274$

[423] Sood A, Midha V, Sood $\mathrm{N}$ et al. Azathioprine versus sulfasalazine in maintenance of remission in severe ulcerative colitis. Indian J Gastroenterol 2003; 22: 79-81

[424] Mate-Jimenez J, Hermida C, Cantero-Perona J et al. 6-mercaptopurine or methotrexate added to prednisone induces and maintains remission in steroid-dependent inflammatory bowel disease. Eur J Gastroenterol Hepatol 2000; 12: 1227-1233

[425] Cassinotti A, Actis GC, Duca P et al. Maintenance treatment with azathioprine in ulcerative colitis: outcome and predictive factors after drug withdrawal. Am J Gastroenterol 2009; 104: 2760-2767

[426] George J, Present DH, Pou R et al. The long-term outcome of ulcerative colitis treated with 6-mercaptopurine. Am J Gastroenterol 1996; 91: $1711-1714$

[427] Fraser AG, Orchard TR, Jewell DP. The efficacy of azathioprine for the treatment of inflammatory bowel disease: a 30 year review. Gut 2002; 50: $485-489$

[428] Ardizzone S, Molteni P, Imbesi V et al. Azathioprine in steroid-resistant and steroid-dependent ulcerative colitis. J Clin Gastroenterol 1997; 25 : $330-333$

[429] Lobo AJ, Foster PN, Burke DA et al. The role of azathioprine in the management of ulcerative colitis. Dis Colon Rectum 1990; 33: 374 377

[430] Khan ZH, Mayberry JF, Spiers N et al. Retrospective case series analysis of patients with inflammatory bowel disease on azathioprine. A district general hospital experience. Digestion 2000; 62: 249-254

[431] Adler DJ, Korelitz BI. The therapeutic efficacy of 6-mercaptopurine in refractory ulcerative colitis. Am J Gastroenterol 1990; 85: 717 - 722

[432] Moreno-Rincon E, Benitez JM, Serrano-Ruiz F] et al. Prognosis of Patients with Ulcerative Colitis in Sustained Remission After Thiopurines Withdrawal. Inflamm Bowel Dis 2015; 21: 1564-1571

[433] Fernandez-Banares F, Bertran X, Esteve-Comas M et al. Azathioprine is useful in maintaining long-term remission induced by intravenous cyclosporine in steroid-refractory severe ulcerative colitis. Am J Gastroenterol 1996; 91: 2498-2499

[434] Ferrante $\mathrm{M}$, Vermeire $\mathrm{S}$, Fidder $\mathrm{H}$ et al. Long-term outcome after infliximab for refractory ulcerative colitis. J Crohns Colitis 2008; 2: 219225

[435] Feagan BG, Sandborn WJ, Lazar A et al. Adalimumab therapy is associated with reduced risk of hospitalization in patients with ulcerative colitis. Gastroenterology 2014; 146: 110 - 118 e3

[436] Colombel JF, Sandborn W], Ghosh S et al. Four-year maintenance treatment with adalimumab in patients with moderately to severely active ulcerative colitis: Data from ULTRA 1, 2, and 3. Am J Gastroenterol 2014; 109: 1771 - 1780

[437] Loftus EV Jr, Colombel JF, Feagan BG et al. Long-term Efficacy of Vedolizumab for Ulcerative Colitis. J Crohns Colitis 2017; 11: 400-411

[438] Feagan BG, Rubin DT, Danese S et al. Efficacy of Vedolizumab Induction and Maintenance Therapy in Patients With Ulcerative Colitis, Regardless of Prior Exposure to Tumor Necrosis Factor Antagonists. Clin Gastroenterol Hepatol 2017; 15: 229-239 e5

[439] Noman M, Ferrante M, Bisschops R et al. Vedolizumab Induces Longterm Mucosal Healing in Patients With Crohn's Disease and Ulcerative Colitis. J Crohns Colitis 2017; 11: 1085 - 1089

[440] Stallmach A, Langbein C, Atreya R et al. Vedolizumab provides clinical benefit over 1 year in patients with active inflammatory bowel disease - a prospective multicenter observational study. Aliment Pharmacol Ther 2016; 44: 1199-1212

[441] Amiot A, Serrero M, Peyrin-Biroulet $L$ et al. One-year effectiveness and safety of vedolizumab therapy for inflammatory bowel disease: a prospective multicentre cohort study. Aliment Pharmacol Ther 2017; 46: $310-321$

[442] Bickston S], Behm BW, Tsoulis D] et al. Vedolizumab for induction and maintenance of remission in ulcerative colitis. Cochrane Database Syst Rev 2014; 8: CD007571

[443] Ungar B, Kopylov U, Engel T et al. Addition of an immunomodulator can reverse antibody formation and loss of response in patients treated with adalimumab. Aliment Pharmacol Ther 2017; 45: 276-282

[444] Matsumoto T, Motoya S, Watanabe K et al. Adalimumab Monotherapy and a Combination with Azathioprine for Crohn's Disease: A Prospective, Randomized Trial. J Crohns Colitis 2016; 10: 1259-1266

[445] Oren R, Arber N, Odes S et al. Methotrexate in chronic active ulcerative colitis: a double-blind, randomized, Israeli multicenter trial. Gastroenterology 1996; 110: 1416-1421

[446] Wahed M, Louis-Auguste JR, Baxter LM et al. Efficacy of methotrexate in Crohn's disease and ulcerative colitis patients unresponsive or intolerant to azathioprine /mercaptopurine. Aliment Pharmacol Ther 2009; 30: 614-620

[447] Wang Y, MacDonald JK, Vandermeer B et al. Methotrexate for maintenance of remission in ulcerative colitis. Cochrane Database Syst Rev 2015; 11: CD007560

[448] Torres ], Boyapati RK, Kennedy NA et al. Systematic Review of Effects of Withdrawal of Immunomodulators or Biologic Agents From Patients With Inflammatory Bowel Disease. Gastroenterology 2015; 149: $1716-1730$

[449] Marits P, Landucci L, Sundin U et al. Trough s-infliximab and antibodies towards infliximab in a cohort of 79 IBD patients with maintenance infliximab treatment. J Crohns Colitis 2014; 8: 881 - 889

[450] Mazor Y, Almog R, Kopylov U et al. Adalimumab drug and antibody levels as predictors of clinical and laboratory response in patients with Crohn's disease. Aliment Pharmacol Ther 2014; 40: 620-628

[451] Cornillie F, Hanauer SB, Diamond RH et al. Postinduction serum infliximab trough level and decrease of $\mathrm{C}$-reactive protein level are associated with durable sustained response to infliximab: a retrospective analysis of the ACCENT I trial. Gut 2014; 63: 1721 -1727 
[452] Pouw MF, Krieckaert CL, Nurmohamed MT et al. Key findings towards optimising adalimumab treatment: the concentration-effect curve. Ann Rheum Dis 2015; 74: 513-518

[453] Seow CH, Newman A, Irwin SP et al. Trough serum infliximab: a predictive factor of clinical outcome for infliximab treatment in acute ulcerative colitis. Gut 2010; 59: 49-54

[454] Moore C, Corbett G, Moss AC. Systematic Review and Meta-Analysis: Serum Infliximab Levels During Maintenance Therapy and Outcomes in Inflammatory Bowel Disease. J Crohns Colitis 2016; 10: 619-625

[455] Roblin X, Marotte H, Rinaudo M et al. Association between pharmacokinetics of adalimumab and mucosal healing in patients with inflammatory bowel diseases. Clin Gastroenterol Hepatol 2014; 12: 80 - 84 e2

[456] Harzallah I, Rigaill J, Williet N et al. Golimumab pharmacokinetics in ulcerative colitis: a literature review. Therap Adv Gastroenterol 2017; 10: $89-100$

[457] Afif W, Loftus EV Jr, Faubion WA et al. Clinical utility of measuring infliximab and human anti-chimeric antibody concentrations in patients with inflammatory bowel disease. Am J Gastroenterol 2010; 105: $1133-1139$

[458] Frederiksen MT, Ainsworth MA, Brynskov J et al. Antibodies against infliximab are associated with de novo development of antibodies to adalimumab and therapeutic failure in infliximab-to-adalimumab switchers with IBD. Inflamm Bowel Dis 2014; 20: 1714-1721

[459] O'Meara S, Nanda KS, Moss AC. Antibodies to infliximab and risk of infusion reactions in patients with inflammatory bowel disease: a systematic review and meta-analysis. Inflamm Bowel Dis 2014; 20 : 1 -6

[460] Ungar B, Chowers Y, Yavzori M et al. The temporal evolution of antidrug antibodies in patients with inflammatory bowel disease treated with infliximab. Gut 2014; 63: 1258-1264

[461] Steenholdt C, Al-khalaf M, Brynskov J et al. Clinical implications of variations in anti-infliximab antibody levels in patients with inflammatory bowel disease. Inflamm Bowel Dis 2012; 18: 2209-2217

[462] Yanai H, Lichtenstein L, Assa A et al. Levels of drug and antidrug antibodies are associated with outcome of interventions after loss of response to infliximab or adalimumab. Clin Gastroenterol Hepatol 2015; $13: 522-530$ e2

[463] Yacoub W, Williet N, Pouillon L et al. Early vedolizumab trough levels predict mucosal healing in inflammatory bowel disease: a multicentre prospective observational study. Aliment Pharmacol Ther 2018; 47: 906-912

[464] Schulze H, Esters P, Hartmann F et al. A prospective cohort study to assess the relevance of vedolizumab drug level monitoring in IBD patients. Scand J Gastroenterol 2018; 53: 670-676

[465] Dreesen E, Verstockt B, Bian S et al. Evidence to Support Monitoring of Vedolizumab Trough Concentrations in Patients With Inflammatory Bowel Diseases. Clin Gastroenterol Hepatol 2018. S15423565(18)30402-6

[466] Baumgart DC, Pintoffl JP, Sturm A et al. Tacrolimus is safe and effective in patients with severe steroid-refractory or steroid-dependent inflammatory bowel disease-a long-term follow-up. Am J Gastroenterol 2006; 101: $1048-1056$

[467] Luthra P, Peyrin-Biroulet L, Ford AC. Systematic review and meta-analysis: opportunistic infections and malignancies during treatment with anti-integrin antibodies in inflammatory bowel disease. Aliment Pharmacol Ther 2015; 41: 1227 - 1236

[468] Brassard P, Bitton A, Suissa A et al. Oral corticosteroids and the risk of serious infections in patients with elderly-onset inflammatory bowel diseases. Am J Gastroenterol 2014; 109: 1795-1802; quiz 1803

[469] Stuck AE, Minder CE, Frey F]. Risk of infectious complications in patients taking glucocorticosteroids. Rev Infect Dis 1989; 11: 954- 963

[470] Dixon WG, Kezouh A, Bernatsky S et al. The influence of systemic glucocorticoid therapy upon the risk of non-serious infection in older patients with rheumatoid arthritis: a nested case-control study. Ann Rheum Dis 2011; 70: 956-960

[471] Jick SS, Lieberman ES, Rahman MU et al. Glucocorticoid use, other associated factors, and the risk of tuberculosis. Arthritis Rheum 2006; 55: $19-26$

[472] Ananthakrishnan AN, McGinley EL. Infection-related hospitalizations are associated with increased mortality in patients with inflammatory bowel diseases. J Crohns Colitis 2013; 7: 107-112

[473] Ha CY. Risks of Infection among the Older Inflammatory Bowel Disease Patients. Curr Treat Options Gastroenterol 2014; 12: 283 - 291

[474] Castle SC. Clinical relevance of age-related immune dysfunction. Clin Infect Dis 2000; 31: 578-585

[475] Strangfeld A, Eveslage M, Schneider M et al. Treatment benefit or survival of the fittest: what drives the time-dependent decrease in serious infection rates under TNF inhibition and what does this imply for the individual patient? Ann Rheum Dis 2011; 70: 1914-1920

[476] Ananthakrishnan AN, Cagan A, Cai T et al. Diabetes and the risk of infections with immunomodulator therapy in inflammatory bowel diseases. Aliment Pharmacol Ther 2015; 41: 1141-1148

[477] Blum HE, Berg T, Tillmann HL et al. Procedures for problematic situations with hepatitis B. Z Gastroenterol 2004; 42: 692-697

[478] Domm S, Cinatl J, Mrowietz U. The impact of treatment with tumour necrosis factor-alpha antagonists on the course of chronic viral infections: a review of the literature. $\mathrm{Br}$ J Dermatol 2008; 159: 1217 1228

[479] Mindikoglu AL, Regev A, Schiff ER. Hepatitis B virus reactivation after cytotoxic chemotherapy: the disease and its prevention. Clin Gastroenterol Hepatol 2006; 4: 1076 - 1081

[480] Esteve M, Saro C, Gonzalez-Huix F et al. Chronic hepatitis B reactivation following infliximab therapy in Crohn's disease patients: need for primary prophylaxis. Gut 2004; 53: $1363-1365$

[481] Lau GK. Hepatitis B reactivation after chemotherapy: two decades of clinical research. Hepatol Int 2008; 2: 152-162

[482] Cornberg M, Protzer U, Petersen J et al. Prophylaxis, diagnosis and therapy of hepatitis B virus infection - the German guideline. Z Gastroenterol 2011; 49: 871 - 930

[483] Diel R, Forssbohm M, Loytved G et al. Empfehlungen für die Umgebungsuntersuchungen bei Tuberkulose. Pneumologie 2007; 61: 440 455

[484] Schoepfer AM, Flogerzi B, Fallegger S et al. Comparison of interferongamma release assay versus tuberculin skin test for tuberculosis screening in inflammatory bowel disease. Am J Gastroenterol 2008; 103: $2799-2806$

[485] Hatzara C, Hadziyannis E, Kandili A et al. Frequent conversion of tuberculosis screening tests during anti-tumour necrosis factor therapy in patients with rheumatic diseases. Ann Rheum Dis 2015; 74: 1848 1853

[486] Thorley-Lawson DA. EBV the prototypical human tumor virus-just how bad is it? J Allergy Clin Immunol 2005; 116: 251 - 261; quiz 262

[487] Thompson MP, Kurzrock R. Epstein-Barr virus and cancer. Clin Cancer Res 2004; 10: $803-821$

[488] Funch DP, Walker AM, Schneider G et al. Ganciclovir and acyclovir reduce the risk of post-transplant lymphoproliferative disorder in renal transplant recipients. Am J Transplant 2005; 5: 2894-2900

[489] Reddy N, Rezvani K, Barrett AJ et al. Strategies to prevent EBV reactivation and posttransplant lymphoproliferative disorders (PTLD) after allogeneic stem cell transplantation in high-risk patients. Biol Blood Marrow Transplant 2011; 17: 591 - 597

[490] Stobaugh DJ, Deepak P, Ehrenpreis ED. Hospitalizations for vaccine preventable pneumonias in patients with inflammatory bowel disease: a 6-year analysis of the Nationwide Inpatient Sample. Clin Exp Gastroenterol 2013; 6: $43-49$ 
[491] Teich N, Klugmann T, Tiedemann A et al. Vaccination coverage in immunosuppressed patients: results of a regional health services research study. Dtsch Arztebl Int 2011; 108: 105-111

[492] Borte S, Liebert UG, Borte M et al. Efficacy of measles, mumps and rubella revaccination in children with juvenile idiopathic arthritis treated with methotrexate and etanercept. Rheumatology (Oxford) 2009; 48: $144-148$

[493] Nguyen DL, Nguyen ET, Bechtold ML. Effect of Immunosuppressive Therapies for the Treatment of Inflammatory Bowel Disease on Response to Routine Vaccinations: A Meta-Analysis. Dig Dis Sci 2015; 60: $2446-2453$

[494] Poppers DM, Scherl EJ. Prophylaxis against Pneumocystis pneumonia in patients with inflammatory bowel disease: toward a standard of care. Inflamm Bowel Dis 2008; 14: 106-113

[495] Cotter TG, Gathaiya N, Catania J et al. Low Risk of Pneumonia From Pneumocystis jirovecii Infection in Patients With Inflammatory Bowel Disease Receiving Immune Suppression. Clin Gastroenterol Hepatol 2017; 15: $850-856$

[496] Long MD, Farraye FA, Okafor PN et al. Increased risk of pneumocystis jiroveci pneumonia among patients with inflammatory bowel disease. Inflamm Bowel Dis 2013; 19: 1018-1024

[497] Stern A, Green H, Paul M et al. Prophylaxis for Pneumocystis pneumonia (PCP) in non-HIV immunocompromised patients. Cochrane Database Syst Rev 2014; 1: CD005590

[498] Messiaen PE, Cuyx S, Dejagere T et al. The role of CD4 cell count as discriminatory measure to guide chemoprophylaxis against Pneumocystis jirovecii pneumonia in human immunodeficiency virus-negative immunocompromised patients: A systematic review. Transpl Infect Dis 2017; 19

[499] Ioannidis JP, Cappelleri JC, Skolnik PR et al. A meta-analysis of the relative efficacy and toxicity of Pneumocystis carinii prophylactic regimens. Arch Intern Med 1996; 156: 177 - 188

[500] Stallmach A, Carstens O. Role of infections in the manifestation or reactivation of inflammatory bowel diseases. Inflamm Bowel Dis 2002; 8: $213-218$

[501] Ananthakrishnan AN, McGinley EL, Binion DG. Excess hospitalisation burden associated with Clostridium difficile in patients with inflammatory bowel disease. Gut 2008; 57: 205-210

[502] Das R, Feuerstadt P, Brandt L]. Glucocorticoids are associated with increased risk of short-term mortality in hospitalized patients with clostridium difficile-associated disease. Am J Gastroenterol 2010; 105: $2040-2049$

[503] Schneeweiss S, Korzenik J, Solomon DH et al. Infliximab and other immunomodulating drugs in patients with inflammatory bowel disease and the risk of serious bacterial infections. Aliment Pharmacol Ther 2009; 30: $253-264$

[504] Singh H, Nugent Z, Yu BN et al. Higher Incidence of Clostridium difficile Infection Among Individuals With Inflammatory Bowel Disease. Gastroenterology 2017; 153: 430-438 e2

[505] Dallal RM, Harbrecht BG, Boujoukas AJ et al. Fulminant Clostridium difficile: an underappreciated and increasing cause of death and complications. Ann Surg 2002; 235: 363 - 372

[506] Asha N], Tompkins D, Wilcox MH. Comparative analysis of prevalence, risk factors, and molecular epidemiology of antibiotic-associated diarrhea due to Clostridium difficile, Clostridium perfringens, and Staphylococcus aureus. J Clin Microbiol 2006; 44: 2785-2791

[507] Crobach MJ, Planche T, Eckert C et al. European Society of Clinical Microbiology and Infectious Diseases: update of the diagnostic guidance document for Clostridium difficile infection. Clin Microbiol Infect 2016; 22 (Suppl. 4): S63 - S81

[508] von Müller L, Halfmann A, Herrmann M. Current data and trends on the development of antibiotic resistance of Clostridium difficile. Bundes- gesundheitsblatt Gesundheitsforschung Gesundheitsschutz 2012; 55: $1410-1417$

[509] Erb S, Frei R, Stranden AM et al. Low sensitivity of fecal toxin A/B enzyme immunoassay for diagnosis of Clostridium difficile infection in immunocompromised patients. Clin Microbiol Infect 2015; 21: 998 e9-998 e15

[510] Vonberg RP, Kuijper EJ, Wilcox MH et al. Infection control measures to limit the spread of Clostridium difficile. Clin Microbiol Infect 2008; 14 (Suppl. 5): 2-20

[511] Fekety R, Silva J, Kauffman C et al. Treatment of antibiotic-associated Clostridium difficile colitis with oral vancomycin: comparison of two dosage regimens. Am J Med 1989; 86: 15-19

[512] Gerding DN, Muto CA, Owens RC et al. Treatment of Clostridium difficile infection. Clin Infect Dis 2008; 46 (Suppl. 1): S32-S42

[513] Kuijper EJ, Wilcox MH. Decreased effectiveness of metronidazole for the treatment of Clostridium difficile infection? Clin Infect Dis 2008; 47: $63-65$

[514] Horton HA, Dezfoli S, Berel D et al. Antibiotics for Treatment of Clostridium difficile Infection in Hospitalized Patients with Inflammatory Bowel Disease. Antimicrob Agents Chemother 2014; 58: 5054 - 5059

[515] Louie T], Miller MA, Mullane KM et al. Fidaxomicin versus vancomycin for Clostridium difficile infection. N Engl ] Med 2011; 364: 422 - 431

[516] Crook DW, Walker AS, Kean Y et al. Fidaxomicin versus vancomycin for Clostridium difficile infection: meta-analysis of pivotal randomized controlled trials. Clin Infect Dis 2012; 55 (Suppl. 2): S93 - S103

[517] Hoegenauer C, Mahida Y, Stallmach A. Open-label study to evaluate the pharmacokinetics of fidaxomicin in inflammatory bowel disease patients with Clostridium difficile infection (the PROFILE study): pharmacokinetics analysis. Journal of Crohn's and Colitis 2017 (Suppl. 1): S276

[518] Nelson RL, Suda KJ, Evans CT. Antibiotic treatment for Clostridium difficile-associated diarrhoea in adults. Cochrane Database Syst Rev 2017; 3: CD004610

[519] Shen NT, Maw A, Tmanova LL et al. Timely Use of Probiotics in Hospitalized Adults Prevents Clostridium difficile Infection: A Systematic Review With Meta-Regression Analysis. Gastroenterology 2017; 152: 1889 - 1900 e9

[520] McFarland LV, Elmer GW, Surawicz CM. Breaking the cycle: treatment strategies for 163 cases of recurrent Clostridium difficile disease. Am J Gastroenterol 2002; 97: 1769-1775

[521] Eiseman B, Silen W, Bascom GS et al. Fecal enema as an adjunct in the treatment of pseudomembranous enterocolitis. Surgery 1958; 44 : $854-859$

[522] van Nood E, Vrieze A, Nieuwdorp M et al. Duodenal infusion of donor feces for recurrent Clostridium difficile. N Engl J Med 2013; 368: 407 415

[523] Fischer M, Kao D, Kelly C et al. Fecal Microbiota Transplantation is Safe and Efficacious for Recurrent or Refractory Clostridium difficile Infection in Patients with Inflammatory Bowel Disease. Inflamm Bowel Dis 2016; 22: $2402-2409$

[524] Khoruts A, Rank KM, Newman KM et al. Inflammatory Bowel Disease Affects the Outcome of Fecal Microbiota Transplantation for Recurrent Clostridium difficile Infection. Clin Gastroenterol Hepatol 2016; 14 : $1433-1438$

[525] Teich N, Weber M, Stallmach A. First Occurrence of Severe Extraintestinal Manifestations of Crohn's Disease Following Faecal Microbiota Transplantation. J Crohns Colitis 2016; 10: 1254-1255

[526] Beaugerie L, Brousse N, Bouvier AM et al. Lymphoproliferative disorders in patients receiving thiopurines for inflammatory bowel disease: a prospective observational cohort study. Lancet 2009; 374: 1617 1625 
[527] Posthuma EF, Westendorp RG, van der Sluys Veer A et al. Fatal infectious mononucleosis: a severe complication in the treatment of Crohn's disease with azathioprine. Gut 1995; 36: 311-313

[528] Garrido Serrano A, Perez Martin F, Guerrero Igea FJ et al. Fatal infectious mononucleosis during azathioprine treatment in Crohn's disease. Gastroenterol Hepatol 2000; 23: 7-8

[529] N'Guyen Y, Andreoletti L, Patey M et al. Fatal Epstein-Barr virus primo infection in a 25-year-old man treated with azathioprine for Crohn's disease. J Clin Microbiol 2009; 47: 1252-1254

[530] Nissen LH, Nagtegaal ID, de Jong DJ et al. Epstein-Barr virus in inflammatory bowel disease: the spectrum of intestinal lymphoproliferative disorders. J Crohns Colitis 2015; 9: 398-403

[531] Fries W, Cottone M, Cascio A. Systematic review: macrophage activation syndrome in inflammatory bowel disease. Aliment Pharmacol Ther 2013; 37: 1033 - 1045

[532] Virdis F, Tacci S, Messina F et al. Hemophagocytic lymphohistiocytosis caused by primary Epstein-Barr virus in patient with Crohn's disease. World J Gastrointest Surg 2013; 5: 306-308

[533] Klein S, Schmidt C, La Rosee P et al. Fulminant gastrointestinal bleeding caused by EBV-triggered hemophagocytic lymphohistiocytosis: report of a case. Z Gastroenterol 2014; 52: 354-359

[534] Brito-Zeron P, Bosch X, Perez-de-Lis M et al. Infection is the major trigger of hemophagocytic syndrome in adult patients treated with biological therapies. Semin Arthritis Rheum 2016; 45: 391 - 399

[535] Cottone M, Pietrosi G, Martorana G et al. Prevalence of cytomegalovirus infection in severe refractory ulcerative and Crohn's colitis. Am J Gastroenterol 2001; 96: 773-775

[536] Kambham N, Vij R, Cartwright CA et al. Cytomegalovirus infection in steroid-refractory ulcerative colitis: a case-control study. Am J Surg Pathol 2004; 28: 365-373

[537] Tandon P, James P, Cordeiro E et al. Diagnostic Accuracy of BloodBased Tests and Histopathology for Cytomegalovirus Reactivation in Inflammatory Bowel Disease: A Systematic Review and Meta-Analysis. Inflamm Bowel Dis 2017; 23: 551-560

[538] Roblin X, Pillet S, Oussalah A et al. Cytomegalovirus load in inflamed intestinal tissue is predictive of resistance to immunosuppressive therapy in ulcerative colitis. Am J Gastroenterol 2011; 106: 2001 - 2008

[539] Zidar N, Ferkolj I, Tepes K et al. Diagnosing cytomegalovirus in patients with inflammatory bowel disease-by immunohistochemistry or polymerase chain reaction? Virchows Arch 2015; 466: 533-539

[540] Thorn M, Rorsman F, Ronnblom A et al. Active cytomegalovirus infection diagnosed by real-time PCR in patients with inflammatory bowel disease: a prospective, controlled observational study (.). Scand J Gastroenterol 2016; 51: 1075 - 1080

[541] Romkens TE, Bulte G], Nissen LH et al. Cytomegalovirus in inflammatory bowel disease: A systematic review. World J Gastroenterol 2016; 22: $1321-1330$

[542] Shukla T, Singh S, Loftus EV Jr et al. Antiviral Therapy in Steroid-refractory Ulcerative Colitis with Cytomegalovirus: Systematic Review and Meta-analysis. Inflamm Bowel Dis 2015; 21: 2718-2725

[543] Kopylov U, Eliakim-Raz N, Szilagy A et al. Antiviral therapy in cytomegalovirus-positive ulcerative colitis: a systematic review and metaanalysis. World J Gastroenterol 2014; 20: 2695 - 2703

[544] Beswick L, Ye B, van Langenberg DR. Toward an Algorithm for the Diagnosis and Management of CMV in Patients with Colitis. Inflamm Bowel Dis 2016; 22: 2966-2976

[545] Kopylov U, Papamichael K, Katsanos K et al. Impact of Infliximab and Cyclosporine on the Risk of Colectomy in Hospitalized Patients with Ulcerative Colitis Complicated by Cytomegalovirus-A Multicenter Retrospective Study. Inflamm Bowel Dis 2017; 23: 1605-1613
[546] Maconi G, Colombo E, Zerbi P et al. Prevalence, detection rate and outcome of cytomegalovirus infection in ulcerative colitis patients requiring colonic resection. Dig Liver Dis 2005; 37: 418-423

[547] Kotton CN, Kumar D, Caliendo AM et al. Updated international consensus guidelines on the management of cytomegalovirus in solidorgan transplantation. Transplantation 2013; 96: $333-360$

[548] Cullen G, Baden RP, Cheifetz AS. Varicella zoster virus infection in inflammatory bowel disease. Inflamm Bowel Dis 2012; 18: 2392 - 2403

[549] Cote-Daigneault J, Peerani F, MacMahon E et al. Management and Prevention of Herpes Zoster in the Immunocompromised Inflammatory Bowel Disease Patient: A Clinical Quandary. Inflamm Bowel Dis 2016; $22: 2538-2547$

[550] Robert Koch-Institut. Tuberkulose. RKI-Ratgeber für Ärzte. 2017 http://www.rki.de/DE/Content/Infekt/EpidBull/Merkblaetter/ Ratgeber_Tuberkulose.htm

[551] Lewinsohn DM, Leonard MK, LoBue PA et al. Official American Thoracic Society/Infectious Diseases Society of America/Centers for Disease Control and Prevention Clinical Practice Guidelines: Diagnosis of Tuberculosis in Adults and Children. Clin Infect Dis 2017; 64: 111 - 115

[552] Julsgaard M, Christensen LA, Gibson PR et al. Concentrations of Adalimumab and Infliximab in Mothers and Newborns, and Effects on Infection. Gastroenterology 2016; 151: 110-119

[553] Lichtenstein GR, Cohen R, Yamashita B et al. Quality of life after proctocolectomy with ileoanal anastomosis for patients with ulcerative colitis. J Clin Gastroenterol 2006; 40: 669-677

[554] Heuschen UA, Heuschen G, Rudek B et al. Long-term quality of life after continence-preserving proctocolectomy for ulcerative colitis and familial adenomatous polyposis. Chirurg 1998; 69: 1052 - 1058

[555] Mark-Christensen A, Erichsen R, Brandsborg S et al. Pouch Failures Following lleal Pouch-anal Anastomosis for Ulcerative Colitis. Colorectal Dis 2018; 20: 44-52

[556] Lovegrove RE, Tilney HS, Remzi FH et al. To divert or not to divert: A retrospective analysis of variables that influence ileostomy omission in ileal pouch surgery. Arch Surg 2011; 146: $82-88$

[557] Mennigen R, Sewald W, Senninger $N$ et al. Morbidity of loop ileostomy closure after restorative proctocolectomy for ulcerative colitis and familial adenomatous polyposis: a systematic review. ] Gastrointest Surg 2014; $18: 2192-2200$

[558] Lovegrove RE, Heriot AG, Constantinides V et al. Meta-analysis of short-term and long-term outcomes of J, W and S ileal reservoirs for restorative proctocolectomy. Colorectal Dis 2007; 9: 310-320

[559] McCormick PH, Guest GD, Clark A] et al. The ideal ileal-pouch design: a long-term randomized control trial of J-vs W-pouch construction. Dis Colon Rectum 2012; 55: 1251 - 1257

[560] Oresland T, Fasth S, Nordgren S et al. A prospective randomized comparison of two different pelvic pouch designs. Scand J Gastroenterol 1990; 25: 986-996

[561] Pal S, Sahni P, Pande GK et al. Outcome following emergency surgery for refractory severe ulcerative colitis in a tertiary care centre in India. BMC Gastroenterol 2005; 5: 39

[562] Heppell J, Farkouh E, Dube $S$ et al. Toxic megacolon. An analysis of 70 cases. Dis Colon Rectum 1986; 29: 789-792

[563] Fowkes L, Krishna K, Menon A et al. Laparoscopic emergency and elective surgery for ulcerative colitis. Colorectal Dis 2008; 10: 373 378

[564] Ziv Y, Fazio VW, Church JM et al. Safety of urgent restorative proctocolectomy with ileal pouch-anal anastomosis for fulminant colitis. Dis Colon Rectum 1995; 38: 345-349

[565] Heyvaert G, Penninckx F, Filez L et al. Restorative proctocolectomy in elective and emergency cases of ulcerative colitis. Int J Colorectal Dis 1994; 9: $73-76$ 
[566] Fazio VW, Ziv Y, Church JM et al. lleal pouch-anal anastomoses complications and function in 1005 patients. Ann Surg 1995; 222: 120 127

[567] Sheth SG, LaMont JT. Toxic megacolon. Lancet 1998; 351: 509-513

[568] Croft A, Walsh A, Doecke J et al. Outcomes of salvage therapy for steroid-refractory acute severe ulcerative colitis: ciclosporin vs. infliximab. Aliment Pharmacol Ther 2013; 38: 294-302

[569] Turner D, Hyams J, Markowitz J et al. Appraisal of the pediatric ulcerative colitis activity index (PUCAI). Inflamm Bowel Dis 2009; 15: 1218 1223

[570] Turner D, Walsh CM, Benchimol El et al. Severe paediatric ulcerative colitis: incidence, outcomes and optimal timing for second-line therapy. Gut 2008; 57: $331-338$

[571] Kimura H, Kunisaki R, Tatsumi K et al. Prolonged Medical Therapy Increases the Risk of Surgical Complications in Patients with Severe Ulcerative Colitis. Dig Surg 2016; 33: 182 - 189

[572] Neumann PA, Mennigen RB, Senninger $N$ et al. Timing of restorative proctocolectomy in patients with medically refractory ulcerative colitis: the patient's point of view. Dis Colon Rectum 2012; 55: 756-761

[573] Feuerstein JD, Akbari M, Tapper EB et al. Systematic review and metaanalysis of third-line salvage therapy with infliximab or cyclosporine in severe ulcerative colitis. Ann Gastroenterol 2016; 29: 341-347

[574] van der Valk ME, Mangen M], Severs M et al. Comparison of Costs and Quality of Life in Ulcerative Colitis Patients with an Ileal Pouch-Anal Anastomosis, lleostomy and Anti-TNFalpha Therapy. J Crohns Colitis 2015; 9: 1016-1023

[575] Heuschkel R, Salvestrini C, Beattie RM et al. Guidelines for the management of growth failure in childhood inflammatory bowel disease. Inflamm Bowel Dis 2008; 14: 839-849

[576] Nicholls RJ, Holt SD, Lubowski DZ. Restorative proctocolectomy with ileal reservoir. Comparison of two-stage vs. three-stage procedures and analysis of factors that might affect outcome. Dis Colon Rectum 1989; 32: 323-326

[577] Selvasekar CR, Cima RR, Larson DW et al. Effect of infliximab on shortterm complications in patients undergoing operation for chronic ulcerative colitis. J Am Coll Surg 2007; 204: 956 - 962; discussion 962-963

[578] Stewart D, Chao A, Kodner I et al. Subtotal colectomy for toxic and fulminant colitis in the era of immunosuppressive therapy. Colorectal Dis 2009; 11: 184-190

[579] Shen B. Impact of preoperative infliximab use on postoperative infectious complications in ulcerative colitis: the price we have to pay? Inflamm Bowel Dis 2008; 14: 1019-1021

[580] Norgard BM, Nielsen J, Qvist N et al. Pre-operative use of anti-TNFalpha agents and the risk of post-operative complications in patients with ulcerative colitis - a nationwide cohort study. Aliment Pharmacol Ther 2012; 35: 1301 - 1309

[581] Mor IJ, Vogel JD, da Luz Moreira A et al. Infliximab in ulcerative colitis is associated with an increased risk of postoperative complications after restorative proctocolectomy. Dis Colon Rectum 2008; 51: 1202 1207; discussion 1207-1210

[582] Yang Z, Wu Q, Wang F et al. Meta-analysis: effect of preoperative infliximab use on early postoperative complications in patients with ulcerative colitis undergoing abdominal surgery. Aliment Pharmacol Ther 2012; 36: $922-928$

[583] Selvaggi F, Pellino G, Canonico S et al. Effect of preoperative biologic drugs on complications and function after restorative proctocolectomy with primary ileal pouch formation: systematic review and metaanalysis. Inflamm Bowel Dis 2015; 21: 79-92

[584] Kulaylat AS, Kulaylat AN, Schaefer EW et al. Association of Preoperative Anti-Tumor Necrosis Factor Therapy With Adverse Postoperative Outcomes in Patients Undergoing Abdominal Surgery for Ulcerative Colitis. JAMA Surg 2017; 152: e171538
[585] Lau C, Dubinsky M, Melmed G et al. The impact of preoperative serum anti-TNFalpha therapy levels on early postoperative outcomes in inflammatory bowel disease surgery. Ann Surg 2015; 261: 487-496

[586] Hait E], Bousvaros A, Schuman M et al. Pouch outcomes among children with ulcerative colitis treated with calcineurin inhibitors before ileal pouch anal anastomosis surgery. J Pediatr Surg 2007; 42: 31 -34; discussion 34-35

[587] Gu J, Stocchi L, Remzi F et al. Intraperitoneal or subcutaneous: does location of the (colo)rectal stump influence outcomes after laparoscopic total abdominal colectomy for ulcerative colitis? Dis Colon Rectum 2013; 56: 615-621

[588] Trickett JP, Tilney HS, Gudgeon AM et al. Management of the rectal stump after emergency sub-total colectomy: which surgical option is associated with the lowest morbidity? Colorectal Dis 2005; 7: 519522

[589] Karch LA, Bauer J], Gorfine SR et al. Subtotal colectomy with Hartmann's pouch for inflammatory bowel disease. Dis Colon Rectum 1995; 38: 635-639

[590] Bartels SA, Gardenbroek T], Aarts M et al. Short-term morbidity and quality of life from a randomized clinical trial of close rectal dissection and total mesorectal excision in ileal pouch-anal anastomosis. Br J Surg 2015; 102: $281-287$

[591] Hicks CW, Hodin RA, Savitt L et al. Does intramesorectal excision for ulcerative colitis impact bowel and sexual function when compared with total mesorectal excision? Am J Surg 2014; 208: 499-504 e4

[592] Rink AD, Radinski I, Vestweber KH. Does mesorectal preservation protect the ileoanal anastomosis after restorative proctocolectomy? J Gastrointest Surg 2009; 13: 120-128

[593] Lovegrove RE, Constantinides VA, Heriot AG et al. A comparison of hand-sewn versus stapled ileal pouch anal anastomosis (IPAA) following proctocolectomy: a meta-analysis of 4183 patients. Ann Surg 2006; $244: 18-26$

[594] Schluender S], Mei L, Yang $\mathrm{H}$ et al. Can a meta-analysis answer the question: is mucosectomy and handsewn or double-stapled anastomosis better in ileal pouch-anal anastomosis? Am Surg 2006; 72: 912 916

[595] Ganschow P, Treiber I, Hinz U et al. Residual rectal mucosa after stapled vs. handsewn ileal J-pouch-anal anastomosis in patients with familial adenomatous polyposis coli (FAP) -a critical issue. Langenbecks Arch Surg 2015; 400: 213-219

[596] Ota H, Yamazaki K, Endoh W et al. Adenocarcinoma arising below an ileoanal anastomosis after restorative proctocolectomy for ulcerative colitis: report of a case. Surg Today 2007; 37: 596 - 599

[597] Alessandroni L, Kohn A, Capaldi M et al. Adenocarcinoma below stapled ileoanal anastomosis after restorative proctocolectomy for ulcerative colitis. Updates Surg 2012; 64: 149-152

[598] Al-Sukhni W, McLeod RS, MacRae H et al. Oncologic outcome in patients with ulcerative colitis associated with dyplasia or cancer who underwent stapled or handsewn ileal pouch-anal anastomosis. Dis Colon Rectum 2010; 53: 1495-1500

[599] Oresland T, Bemelman WA, Sampietro GM et al. European evidence based consensus on surgery for ulcerative colitis. J Crohns Colitis 2015; 9: $4-25$

[600] Tekkis PP, Fazio VW, Lavery IC et al. Evaluation of the learning curve in ileal pouch-anal anastomosis surgery. Ann Surg 2005; 241: 262-268

[601] Parc Y, Reboul-Marty J, Lefevre JH et al. Restorative Proctocolectomy and Ileal Pouch-anal Anastomosis. Ann Surg 2015; 262: 849-853; discussion 853-854

[602] Burns EM, Bottle A, Aylin P et al. Volume analysis of outcome following restorative proctocolectomy. Br J Surg 2011; 98: 408-417

[603] Raval M], Schnitzler M, O'Connor Bl et al. Improved outcome due to increased experience and individualized management of leaks after ileal pouch-anal anastomosis. Ann Surg 2007; 246: $763-770$ 
[604] Borjesson L, Willen R, Haboubi N et al. The risk of dysplasia and cancer in the ileal pouch mucosa after restorative proctocolectomy for ulcerative proctocolitis is low: a long-term term follow-up study. Colorectal Dis 2004; 6: 494-498

[605] Pachler FR, Brandsborg SB, Laurberg S. Paradoxical Impact of Ileal Pouch-Anal Anastomosis on Male and Female Fertility in Patients With Ulcerative Colitis. Dis Colon Rectum 2017; 60: 603-607

[606] Uzzan M, Cosnes ], Amiot A et al. Long-term Follow-up After Ileorectal Anastomosis for Ulcerative Colitis: A GETAID/GETAID Chirurgie Multicenter Retrospective Cohort of 343 Patients. Ann Surg 2017; 266: $1029-1034$

[607] Borjesson L, Lundstam U, Oresland T et al. The place for colectomy and ileorectal anastomosis: a valid surgical option for ulcerative colitis? Tech Coloproctol 2006; 10: 237-241; discussion 241

[608] da Luz Moreira A, Kiran RP, Lavery I. Clinical outcomes of ileorectal anastomosis for ulcerative colitis. Br ] Surg 2010; 97: 65-69

[609] Soravia C, O’Connor BI, Berk T et al. Functional outcome of conversion of ileorectal anastomosis to ileal pouch-anal anastomosis in patients with familial adenomatous polyposis and ulcerative colitis. Dis Colon Rectum 1999; 42: $903-908$

[610] Nessar G, Fazio VW, Tekkis P et al. Long-term outcome and quality of life after continent ileostomy. Dis Colon Rectum 2006; 49: 336-344

[611] Berndtsson I, Lindholm E, Ekman I. Thirty years of experience living with a continent ileostomy: bad restrooms-not my reservoir-decide my life. J Wound Ostomy Continence Nurs 2005; 32: 321 - 326; quiz 327-328

[612] Litle VR, Barbour S, Schrock TR et al. The continent ileostomy: longterm durability and patient satisfaction. J Gastrointest Surg 1999; 3: $625-632$

[613] Lian L, Fazio VW, Remzi FH et al. Outcomes for patients undergoing continent ileostomy after a failed ileal pouch-anal anastomosis. Dis Colon Rectum 2009; 52: 1409 - 1414; discussion 4414-4416

[614] Uzzan M, Kirchgesner ], Oubaya N et al. Risk of Rectal Neoplasia after Colectomy and Ileorectal Anastomosis for Ulcerative Colitis. J Crohns Colitis 2017; 11: $930-935$

[615] Kuiper T, Vlug MS, van den Broek FJ et al. The prevalence of dysplasia in the ileoanal pouch following restorative proctocolectomy for ulcerative colitis with associated dysplasia. Colorectal Dis 2012; 14: 469-473

[616] Martense S, Dunker MS, Slors JF et al. Hand-assisted laparoscopic versus open restorative proctocolectomy with ileal pouch anal anastomosis: a randomized trial. Ann Surg 2004; 240: 984-991; discussion 991-992

[617] Tilney HS, Lovegrove RE, Heriot AG et al. Comparison of short-term outcomes of laparoscopic vs open approaches to ileal pouch surgery. Int J Colorectal Dis 2007; 22: 531 - 542

[618] Ahmed AliU, Keus F, Heikens JT et al. Open versus laparoscopic (assisted) ileo pouch anal anastomosis for ulcerative colitis and familial adenomatous polyposis. Cochrane Database Syst Rev 2009; 21: CD006267

[619] Bartels SA, Gardenbroek T], Ubbink DT et al. Systematic review and meta-analysis of laparoscopic versus open colectomy with end ileostomy for non-toxic colitis. Br J Surg 2013; 100: 726-733

[620] Wu X], He XS, Zhou XY et al. The role of laparoscopic surgery for ulcerative colitis: systematic review with meta-analysis. Int J Colorectal Dis 2010; 25: $949-957$

[621] Beyer-Berjot L, Maggiori L, Birnbaum D et al. A total laparoscopic approach reduces the infertility rate after ileal pouch-anal anastomosis: a 2-center study. Ann Surg 2013; 258: 275-282

[622] Bartels SA, Vlug MS, Henneman D et al. Less adhesiolysis and hernia repair during completion proctocolectomy after laparoscopic emergency colectomy for ulcerative colitis. Surg Endosc 2012; 26: 368 373
[623] Indar AA, Efron JE, Young-Fadok TM. Laparoscopic ileal pouch-anal anastomosis reduces abdominal and pelvic adhesions. Surg Endosc 2009; 23: $174-177$

[624] Bartels SA, D’Hoore A, Cuesta MA et al. Significantly increased pregnancy rates after laparoscopic restorative proctocolectomy: a crosssectional study. Ann Surg 2012; 256: 1045-1048

[625] Benlice C, Stocchi L, Costedio M et al. Laparoscopic IPAA is not associated with decreased rates of incisional hernia and small-bowel obstruction when compared with open technique: long-term follow-up of a case-matched study. Dis Colon Rectum 2015; 58: 314-320

[626] Holubar SD, Larson DW, Dozois EJ et al. Minimally invasive subtotal colectomy and ileal pouch-anal anastomosis for fulminant ulcerative colitis: a reasonable approach? Dis Colon Rectum 2009; 52: 187-192

[627] Chung TP, Fleshman JW, Birnbaum EH et al. Laparoscopic vs. open total abdominal colectomy for severe colitis: impact on recovery and subsequent completion restorative proctectomy. Dis Colon Rectum 2009; 52: $4-10$

[628] Telem DA, Vine AJ, Swain G et al. Laparoscopic subtotal colectomy for medically refractory ulcerative colitis: the time has come. Surg Endosc 2010; 24: $1616-1620$

[629] Geboes K, Colombel JF, Greenstein A et al. Indeterminate colitis: a review of the concept-what's in a name? Inflamm Bowel Dis 2008; 14: $850-857$

[630] Pishori T, Dinnewitzer A, Zmora O et al. Outcome of patients with indeterminate colitis undergoing a double-stapled ileal pouch-anal anastomosis. Dis Colon Rectum 2004; 47: 717 - 721

[631] Delaney CP, Remzi FH, Gramlich T et al. Equivalent function, quality of life and pouch survival rates after ileal pouch-anal anastomosis for indeterminate and ulcerative colitis. Ann Surg 2002; 236: 43-48

[632] Reese GE, Lovegrove RE, Tilney HS et al. The effect of Crohn's disease on outcomes after restorative proctocolectomy. Dis Colon Rectum 2007; 50: $239-250$

[633] Malaty HM, Fan X, Opekun AR et al. Rising incidence of inflammatory bowel disease among children: a 12-year study. J Pediatr Gastroentero Nutr 2010; 50: 27-31

[634] Svaninger G, Nordgren S, Oresland T et al. Incidence and characteristics of pouchitis in the Kock continent ileostomy and the pelvic pouch. Scand J Gastroenterol 1993; 28: 695-700

[635] Rauh SM, Schoetz D] Jr, Roberts PL et al. Pouchitis-is it a wastebasket diagnosis? Dis Colon Rectum 1991; 34: 685-689

[636] Sandborn W], Tremaine WJ, Batts KP et al. Pouchitis after ileal pouchanal anastomosis: a Pouchitis Disease Activity Index. Mayo Clin Proc 1994; 69: 409-415

[637] Tiainen J, Matikainen M. Long-term clinical outcome and anemia after restorative proctocolectomy for ulcerative colitis. Scand J Gastroenterol 2000; 35: $1170-1173$

[638] Meagher AP, Farouk R, Dozois RR et al. J ileal pouch-anal anastomosis for chronic ulcerative colitis: complications and long-term outcome in 1310 patients. $\mathrm{Br}$ J Surg 1998; 85: $800-803$

[639] Hurst RD, Chung TP, Rubin M et al. The implications of acute pouchitis on the long-term functional results after restorative proctocolectomy. Inflamm Bowel Dis 1998; 4: 280 - 284

[640] Tiainen J, Matikainen M, Aitola P et al. Histological and macroscopic changes in the pelvic pouch: long-term follow up after restorative proctocolectomy for ulcerative colitis (UC). Colorectal Dis 2001; 3: $28-32$

[641] Tiainen J, Matikainen M. Health-related quality of life after ileal Jpouch-anal anastomosis for ulcerative colitis: long-term results. Scand J Gastroenterol 1999; 34: 601-605

[642] Seidel SA, Peach SE, Newman M et al. lleoanal pouch procedures: clinical outcomes and quality-of-life assessment. Am Surg 1999; 65: $40-46$ 
[643] Heuschen UA, Autschbach F, Allemeyer EH et al. Long-term follow-up after ileoanal pouch procedure: algorithm for diagnosis, classification, and management of pouchitis. Dis Colon Rectum 2001; 44: 487-499

[644] Stahlberg D, Gullberg K, Liljeqvist L et al. Pouchitis following pelvic pouch operation for ulcerative colitis. Incidence, cumulative risk, and risk factors. Dis Colon Rectum 1996; 39: 1012-1018

[645] Lohmuller JL, Pemberton JH, Dozois RR et al. Pouchitis and extraintestinal manifestations of inflammatory bowel disease after ileal pouchanal anastomosis. Ann Surg 1990; 211: 622 - 627; discussion 627-629

[646] Nicholls RJ, Banerjee AK. Pouchitis: risk factors, etiology, and treatment. World J Surg 1998; 22: 347-351

[647] Penna C, Dozois R, Tremaine W et al. Pouchitis after ileal pouch-anal anastomosis for ulcerative colitis occurs with increased frequency in patients with associated primary sclerosing cholangitis. Gut 1996; 38 : 234-239

[648] Shen B, Achkar JP, Lashner BA et al. Irritable pouch syndrome: a new category of diagnosis for symptomatic patients with ileal pouch-anal anastomosis. Am J Gastroenterol 2002; 97: 972 -977

[649] Thoeni RF, Fell SC, Engelstad B et al. Ileoanal pouches: comparison of $\mathrm{CT}$, scintigraphy, and contrast enemas for diagnosing postsurgical complications. Am J Roentgenol 1990; 154: 73-78

[650] Libicher M, Scharf ], Wunsch A et al. MRI of pouch-related fistulas in ulcerative colitis after restorative proctocolectomy. J Comput Assist Tomogr 1998; 22: $664-668$

[651] Hrung JM, Levine MS, Rombeau JL et al. Total proctocolectomy and ileoanal pouch: the role of contrast studies for evaluating postoperative leaks. Abdom Imaging 1998; 23: 375-379

[652] Solomon M], McLeod RS, O'Connor Bl et al. Assessment of peripouch inflammation after ileoanal anastomosis using endoluminal ultrasonography. Dis Colon Rectum 1995; 38: 182-187

[653] Segal JP, Ding NS, Worley G et al. Systematic review with meta-analysis: the management of chronic refractory pouchitis with an evidencebased treatment algorithm. Aliment Pharmacol Ther 2017; 45: 581 592

[654] Sandborn W, McLeod R, Jewell D. Pharmacotherapy for inducing and maintaining remission in pouchitis. Cochrane Database Syst Rev 2000; 2: CD001176

[655] Sandborn W], McLeod R, Jewell DP. Medical therapy for induction and maintenance of remission in pouchitis: a systematic review. Inflamm Bowel Dis 1999; 5: 33-39

[656] Singh S, Stroud AM, Holubar SD et al. Treatment and prevention of pouchitis after ileal pouch-anal anastomosis for chronic ulcerative colitis. Cochrane Database Syst Rev 2015; 23: CD001176

[657] Mimura T, Rizzello F, Helwig U et al. Four-week open-label trial of metronidazole and ciprofloxacin for the treatment of recurrent or refractory pouchitis. Aliment Pharmacol Ther 2002; 16: 909-917

[658] Madden MV, McIntyre AS, Nicholls RJ. Double-blind crossover trial of metronidazole versus placebo in chronic unremitting pouchitis. Dig Dis Sci 1994; 39: 1193-1196

[659] Shen B, Achkar JP, Lashner BA et al. A randomized clinical trial of ciprofloxacin and metronidazole to treat acute pouchitis. Inflamm Bowel Dis 2001; 7: $301-305$

[660] Hurst RD, Molinari M, Chung TP et al. Prospective study of the incidence, timing and treatment of pouchitis in 104 consecutive patients after restorative proctocolectomy. Arch Surg 1996; 131: 497 -500; discussion 501-502

[661] Shen B, Fazio VW, Remzi FH et al. Combined ciprofloxacin and tinidazole therapy in the treatment of chronic refractory pouchitis. Dis Colon Rectum 2007; 50: $498-508$

[662] Gionchetti P, Rizzello F, Venturi A et al. Antibiotic combination therapy in patients with chronic, treatment-resistant pouchitis. Aliment Pharmacol Ther 1999; 13: 713-718
[663] Nygaard K, Bergan T, Bjorneklett A et al. Topical metronidazole treatment in pouchitis. Scand J Gastroenterol 1994; 29: 462-467

[664] Abdelrazeq AS, Kelly SM, Lund JN et al. Rifaximin-ciprofloxacin combination therapy is effective in chronic active refractory pouchitis. Colorectal Dis 2005; 7: 182-186

[665] Gionchetti P, Rizzello F, Poggioli G et al. Oral budesonide in the treatment of chronic refractory pouchitis. Aliment Pharmacol Ther 2007; 25: $1231-1236$

[666] Sambuelli A, Boerr L, Negreira S et al. Budesonide enema in pouchitisa double-blind, double-dummy, controlled trial. Aliment Pharmacol Ther 2002; 16: 27 -34

[667] Herfarth HH, Long MD, Isaacs KL. Use of Biologics in Pouchitis: A Systematic Review. J Clin Gastroenterol 2015; 49: 647-654

[668] Kelly OB, Rosenberg M, Tyler AD et al. Infliximab to Treat Refractory Inflammation After Pelvic Pouch Surgery for Ulcerative Colitis. J Crohns Colitis 2016; 10: 410-417

[669] Li Y, Lopez R, Queener E et al. Adalimumab therapy in Crohn's disease of the ileal pouch. Inflamm Bowel Dis 2012; 18: 2232-2239

[670] Barreiro-de Acosta M, Garcia-Bosch O, Gordillo J et al. Efficacy of adalimumab rescue therapy in patients with chronic refractory pouchitis previously treated with infliximab: a case series. Eur J Gastroenterol Hepatol 2012; 24: 756-758

[671] Mir F, Yousef MH, Partyka EK et al. Successful treatment of chronic refractory pouchitis with vedolizumab. Int J Colorectal Dis 2017; 32: $1517-1518$

[672] Tran-Minh ML, Allez M, Gornet JM. Successful Treatment With Ustekinumab for Chronic Refractory Pouchitis. J Crohns Colitis 2017; 11: 1156

[673] Winter TA, Dalton HR, Merrett MN et al. Cyclosporin A retention enemas in refractory distal ulcerative colitis and 'pouchitis'. Scand J Gastroenterol 1993; 28: 701-704

[674] Fang S, Kraft CS, Dhere T et al. Successful treatment of chronic Pouchitis utilizing fecal microbiota transplantation (FMT): a case report. Int J Colorectal Dis 2016; 31: 1093 - 1094

[675] Schmid M, Frick JS, Malek N et al. Successful treatment of pouchitis with Vedolizumab, but not fecal microbiota transfer (FMT), after proctocolectomy in ulcerative colitis. Int J Colorectal Dis 2017; 32: 597 598

[676] Miner P, Wedel M, Bane B et al. An enema formulation of alicaforsen, an antisense inhibitor of intercellular adhesion molecule-1, in the treatment of chronic, unremitting pouchitis. Aliment Pharmacol Ther 2004; 19: $281-286$

[677] Shen B, Remzi FH, Lopez AR et al. Rifaximin for maintenance therapy in antibiotic-dependent pouchitis. BMC Gastroenterol 2008; 8: 26

[678] Greuter T, Biedermann L, Rogler $G$ et al. Alicaforsen, an antisense inhibitor of ICAM-1, as treatment for chronic refractory pouchitis after proctocolectomy: A case series. United European Gastroenterol J 2016; 4: $97-104$

[679] Shen B, Remzi FH, Lavery IC et al. Administration of adalimumab in the treatment of Crohn's disease of the ileal pouch. Aliment Pharmacol Ther 2009; 29: 519-526

[680] Holubar SD, Cima RR, Sandborn W] et al. Treatment and prevention of pouchitis after ileal pouch-anal anastomosis for chronic ulcerative colitis. Cochrane Database Syst Rev 2010; 16: CD001176

[681] Mimura T, Rizzello F, Helwig U et al. Once daily high dose probiotic therapy (VSL\#3) for maintaining remission in recurrent or refractory pouchitis. Gut 2004; 53: $108-114$

[682] Gionchetti P, Rizzello F, Venturi A et al. Oral bacteriotherapy as maintenance treatment in patients with chronic pouchitis: a double-blind, placebo-controlled trial. Gastroenterology 2000; 119: 305-309 
[683] Zhu H, Wu XR, Queener E et al. Clinical value of surveillance pouchoscopy in asymptomatic ileal pouch patients with underlying inflammatory bowel disease. Surg Endosc 2013; 27: 4325-4332

[684] Gullberg K, Lindforss U, Zetterquist $\mathrm{H}$ et al. Cancer risk assessment in long-standing pouchitis. DNA aberrations are rare in transformed neoplastic pelvic pouch mucosa. Int J Colorectal Dis 2002; 17: 92 - 97

[685] Hashimoto T, Itabashi M, Ogawa S et al. Treatment strategy for preventing pouchitis as a postoperative complication of ulcerative colitis: the significance of the management of cuffitis. Surg Today 2014; 44 : $1730-1734$

[686] Shen B, Lashner BA, Bennett AE et al. Treatment of rectal cuff inflammation (cuffitis) in patients with ulcerative colitis following restorative proctocolectomy and ileal pouch-anal anastomosis. Am J Gastroenterol 2004; 99: 1527-1531

[687] Shen B, Sanmiguel C, Bennett AE et al. Irritable pouch syndrome is characterized by visceral hypersensitivity. Inflamm Bowel Dis 2011; 17: 994- 1002

[688] Hou JK, Abraham B, El-Serag H. Dietary intake and risk of developing inflammatory bowel disease: a systematic review of the literature. Am J Gastroenterol 2011; 106: 563-573

[689] Kruis W, Fric P, Pokrotnieks ] et al. Maintaining remission of ulcerative colitis with the probiotic Escherichia coli Nissle 1917 is as effective as with standard mesalazine. Gut 2004; 53: 1617-1623

[690] Yoshimatsu Y, Yamada A, Furukawa R et al. Effectiveness of probiotic therapy for the prevention of relapse in patients with inactive ulcerative colitis. World J Gastroenterol 2015; 21: 5985 - 5994

[691] Gionchetti P, Rizzello F, Morselli C et al. High-dose probiotics for the treatment of active pouchitis. Dis Colon Rectum 2007; 50: 2075 2082; discussion 2082-2084

[692] Klement E, Reif S. Breastfeeding and risk of inflammatory bowel disease. Am J Clin Nutr 2005; 82: 486

[693] Halmos EP, Muir JG, Barrett JS et al. Diarrhoea during enteral nutrition is predicted by the poorly absorbed short-chain carbohydrate (FODMAP) content of the formula. Aliment Pharmacol Ther 2010; 32: 925 933

[694] Gearry RB, Richardson AK, Frampton CM et al. Population-based cases control study of inflammatory bowel disease risk factors. J Gastroenterol Hepatol 2010; 25: 325-333

[695] Zocco MA, dal Verme LZ, Cremonini F et al. Efficacy of Lactobacillus GG in maintaining remission of ulcerative colitis. Aliment Pharmacol Ther 2006; 23: $1567-1574$

[696] Kappelman MD, Bousvaros A. Nutritional concerns in pediatric inflammatory bowel disease patients. Mol Nutr Food Res 2008; 52: 867 - 874

[697] Nguyen GC, Munsell M, Harris ML. Nationwide prevalence and prognostic significance of clinically diagnosable protein-calorie malnutrition in hospitalized inflammatory bowel disease patients. Inflamm Bowel Dis 2008; 14: $1105-1111$

[698] Massironi S, Rossi RE, Cavalcoli FA et al. Nutritional deficiencies in inflammatory bowel disease: therapeutic approaches. Clin Nutr 2013; 32: $904-910$

[699] Han YM, Yoon H, Lim S et al. Risk Factors for Vitamin D, Zinc, and Selenium Deficiencies in Korean Patients with Inflammatory Bowel Disease. Gut Liver 2017; 11: 363 - 369

[700] Oikonomou IK, Fazio VW, Remzi FH et al. Risk factors for anemia in patients with ileal pouch-anal anastomosis. Dis Colon Rectum 2007; 50: $69-74$

[701] Pastrana RJ, Torres EA, Arroyo JM et al. Iron-deficiency anemia as presentation of pouchitis. J Clin Gastroenterol 2007; 41: 41 - 44

[702] Prince AC, Myers CE, Joyce T et al. Fermentable Carbohydrate Restriction (Low FODMAP Diet) in Clinical Practice Improves Functional Gastrointestinal Symptoms in Patients with Inflammatory Bowel Disease. Inflamm Bowel Dis 2016; 22: 1129-1136
[703] M'Koma AE. Follow-up results of hematology data before and after restorative proctocolectomy. Clinical outcome. Dis Colon Rectum 1994; 37: $932-937$

[704] Bischoff SC, Koletzko B, Lochs H et al. Klinische Ernährung in der Gastroenterologie (Teil 4) - Chronisch-entzündliche Darmerkrankungen. Aktuel Ernahrungsmed 2014; 39: e72-e98

[705] Turner D, Zlotkin SH, Shah PS et al. Omega 3 fatty acids (fish oil) for maintenance of remission in Crohn's disease. Cochrane Database Syst Rev 2009; 21: CD006320

[706] Turner D, Shah PS, Steinhart AH et al. Maintenance of remission in inflammatory bowel disease using omega-3 fatty acids (fish oil): a systematic review and meta-analyses. Inflamm Bowel Dis 2011; 17: 336 345

[707] Klement E, Cohen RV, Boxman J et al. Breastfeeding and risk of inflammatory bowel disease: a systematic review with meta-analysis. Am J Clin Nutr 2004; 80: 1342 - 1352

[708] Akobeng AK, Elawad M, Gordon M. Glutamine for induction of remission in Crohn's disease. Cochrane Database Syst Rev 2016; 2: CD007348

[709] Lewis JD, Abreu MT. Diet as a Trigger or Therapy for Inflammatory Bowel Diseases. Gastroenterology 2017; 152: 398-414 e6

[710] Lochs H, Dejong C, Hammarqvist F et al. ESPEN Guidelines on Enteral Nutrition: Gastroenterology. Clin Nutr 2006; 25: 260-274

[711] Schulz RJ, Bischoff SC, Koletzko B et al. Gastroenterology - Guidelines on Parenteral Nutrition, Chapter 15. Ger Med Sci 2009; 7: Doc13

[712] Forbes A, Escher J, Hebuterne X et al. ESPEN guideline: Clinical nutrition in inflammatory bowel disease. Clin Nutr 2017; 36: 321 - 347

[713] Song HK, Buzby GP. Nutritional support for Crohn's disease. Surg Clin North Am 2001; 81: $103-115$, viii

[714] Nguyen DL, Parekh NK, Jamal MM. National Trends and Outcomes of Inflammatory Bowel Disease Patients Requiring in-Hospital Total Parenteral Nutrition Support. Gastroenterology 2013; 144: S749-S749

[715] Bischoff SC, Fleig W. Ulcerative colitis. Nutrition. Z Gastroenterol 2004 42: $998-1002$

[716] Stein J. Leitlinien Morbus Crohn. Z Gastroenterol 2003; 41: 62 - 68

[717] Lee D, Albenberg L, Compher C et al. Diet in the pathogenesis and treatment of inflammatory bowel diseases. Gastroenterology 2015; 148: $1087-1106$

[718] Martin J, Radeke HH, Dignass A et al. Current evaluation and management of anemia in patients with inflammatory bowel disease. Expert Rev Gastroenterol Hepatol 2017; 11: 19-32

[719] Evstatiev R, Marteau P, Iqbal T et al. FERGlcor, a randomized controlled trial on ferric carboxymaltose for iron deficiency anemia in inflammatory bowel disease. Gastroenterology 2011; 141: 846-853 e1-2

[720] Wolman SL, Anderson GH, Marliss EB et al. Zinc in total parenteral nutrition: requirements and metabolic effects. Gastroenterology 1979; 76: $458-467$

[721] Jeejeebhoy K. Zinc: an essential trace element for parenteral nutrition. Gastroenterology 2009; 137: S7 - S12

[722] Schölmerich J, Freudemann A, Köttgen E et al. Bioavailability of zinc from zinc-histidine complexes. I. Comparison with zinc sulfate in healthy men. Am J Clin Nutr 1987; 45: 1480-1486

[723] Rossi RE, Whyand T, Murray CD et al. The role of dietary supplements in inflammatory bowel disease: a systematic review. Eur J Gastroenterol Hepatol 2016; 28: 1357 - 1364

[724] Fessler TA. Trace elements in parenteral nutrition: a practical guide for dosage and monitoring for adult patients. Nutr Clin Pract 2013; 28 : $722-729$

[725] Kruis W, Phuong Nguyen G. Iron Deficiency, Zinc, Magnesium, Vitamin Deficiencies in Crohn's Disease: Substitute or Not? Dig Dis 2016; 34 : $105-111$ 
[726] Hlavaty T, Krajcovicova A, Payer ]. Vitamin D therapy in inflammatory bowel diseases: who, in what form, and how much? J Crohns Colitis 2015; 9: $198-209$

[727] Raftery T, O’Sullivan M. Optimal vitamin D levels in Crohn's disease: a review. Proc Nutr Soc 2015; 74: 56-66

[728] Lee JA, Hwang JS, Hwang IT et al. Low vitamin D levels are associated with both iron deficiency and anemia in children and adolescents. Pediatr Hematol Oncol 2015; 32: $99-108$

[729] Laake KO, Bjorneklett A, Aamodt G et al. Outcome of four weeks' intervention with probiotics on symptoms and endoscopic appearance after surgical reconstruction with a J-configurated ileal-pouch-analanastomosis in ulcerative colitis. Scand ] Gastroenterol 2005; 40: 43 51

[730] Ng SC, Plamondon S, Kamm MA et al. Immunosuppressive effects via human intestinal dendritic cells of probiotic bacteria and steroids in the treatment of acute ulcerative colitis. Inflamm Bowel Dis 2010; 16: $1286-1298$

[731] Laake KO, Line PD, Aabakken L et al. Assessment of Mucosal Inflammation and Circulation in Response to Probiotics in Patients Operated with Ileal Pouch Anal Anastomosis for Ulcerative Colitis. Scand J Gastroenterol 2003; 38: 409-414

[732] Higgens CS, Keighley MR, Allan RN. Impact of preoperative weight loss and body composition changes on postoperative outcome in surgery for inflammatory bowel disease. Gut 1984; 25: 732-736

[733] Lindor KD, Fleming CR, Ilstrup DM. Preoperative nutritional status and other factors that influence surgical outcome in patients with Crohn's disease. Mayo Clin Proc 1985; 60: 393 -396

[734] Rombeau JL, Barot LR, Williamson CE et al. Preoperative total parenteral nutrition and surgical outcome in patients with inflammatory bowel disease. Am J Surg 1982; 143: 139-143

[735] Schwartz E. Perioperative Parenteral Nutrition in Adults With Inflammatory Bowel Disease: A Review of the Literature. Nutr Clin Pract 2016; 31: 159-170

[736] Weimann A, Braga M, Harsanyi L et al. ESPEN Guidelines on Enteral Nutrition: Surgery including organ transplantation. Clin Nutr 2006; 25 : $224-244$

[737] Sorensen J, Kondrup J, Prokopowicz J et al. EuroOOPS: an international, multicentre study to implement nutritional risk screening and evaluate clinical outcome. Clin Nutr 2008; 27: 340 -349

[738] Weimann A, Breitenstein S, Breuer JP et al. Clinical nutrition in surgery. Guidelines of the German Society for Nutritional Medicine. Chirurg 2014; 85: $320-326$

[739] Stein J. Kurzdarmsyndrom - Chronisches Darmversagen. In: E.D. S, ed. Entzündliche Darmerkrankungen. Stuttgart: Schattauer; 2015: 361 386

[740] M'Koma AE, Wise PE, Schwartz DA et al. Prevalence and outcome of anemia after restorative proctocolectomy: a clinical literature review. Dis Colon Rectum 2009; 52: 726 - 739

[741] Stein J, Connor S, Virgin G et al. Anemia and iron deficiency in gastrointestinal and liver conditions. World J Gastroenterol 2016; 22: 7908 7925

[742] M'Koma AE. Serum biochemical evaluation of patients with functional pouches ten to 20 years after restorative proctocolectomy. Int J Colorectal Dis 2006; $21: 711-720$

[743] Khanna R, Wu X, Shen B. Low levels of vitamin D are common in patients with ileal pouches irrespective of pouch inflammation. J Crohns Colitis 2013; 7: 525-533

[744] Kuisma J, Nuutinen H, Luukkonen P et al. Long term metabolic consequences of ileal pouch-anal anastomosis for ulcerative colitis. Am J Gastroenterol 2001; 96: 3110-3116

[745] Wieland LS, Manheimer E, Berman BM. Development and classification of an operational definition of complementary and alternative medici- ne for the Cochrane collaboration. Altern Ther Health Med 2011; 17: $50-59$

[746] Barnes PM, Bloom B, Nahin RL. Complementary and alternative medicine use among adults and children: United States, 2007. Natl Health Stat Report 2008; 10: $1-23$

[747] Bensoussan M, Jovenin N, Garcia B et al. Complementary and alternative medicine use by patients with inflammatory bowel disease: results from a postal survey. Gastroenterol Clin Biol 2006; 30: 14-23

[748] Burgmann T, Rawsthorne P, Bernstein CN. Predictors of alternative and complementary medicine use in inflammatory bowel disease: do measures of conventional health care utilization relate to use? Am J Gastroenterol 2004; 99: 889-893

[749] Hilsden RJ, Meddings JB, Verhoef MJ. Complementary and alternative medicine use by patients with inflammatory bowel disease: An Internet survey. Can J Gastroenterol 1999; 13: 327-332

[750] Hilsden RJ, Verhoef MJ, Rasmussen $\mathrm{H}$ et al. Use of complementary and alternative medicine by patients with inflammatory bowel disease. Inflamm Bowel Dis 2011; 17: 655-662

[751] Kong SC, Hurlstone DP, Pocock CY et al. The Incidence of self-prescribed oral complementary and alternative medicine use by patients with gastrointestinal diseases. J Clin Gastroenterol 2005; 39: 138-141

[752] Langhorst ], Anthonisen IB, Steder-Neukamm U et al. Amount of systemic steroid medication is a strong predictor for the use of complementary and alternative medicine in patients with inflammatory bowel disease: results from a German national survey. Inflamm Bowel Dis 2005; 11: $287-295$

[753] Langhorst J, Wulfert $\mathrm{H}$, Lauche $\mathrm{R}$ et al. Systematic review of complementary and alternative medicine treatments in inflammatory bowel diseases. J Crohns Colitis 2015; 9: 86-106

[754] Elsenbruch S, Langhorst ], Popkirowa K et al. Effects of mind-body therapy on quality of life and neuroendocrine and cellular immune functions in patients with ulcerative colitis. Psychother Psychosom 2005; 74: 277-287

[755] Langhorst J, Mueller T, Luedtke R et al. Effects of a comprehensive lifestyle modification program on quality-of-life in patients with ulcerative colitis: a twelve-month follow-up. Scand J Gastroenterol 2007; 42: $734-745$

[756] Berrill JW, Sadlier M, Hood K et al. Mindfulness-based therapy for inflammatory bowel disease patients with functional abdominal symptoms or high perceived stress levels. J Crohns Colitis 2014; 8: 945-955

[757] Jedel S, Hoffman A, Merriman P et al. A randomized controlled trial of mindfulness-based stress reduction to prevent flare-up in patients with inactive ulcerative colitis. Digestion 2014; 89: 142-155

[758] Mizrahi MC, Reicher-Atir R, Levy S et al. Effects of guided imagery with relaxation training on anxiety and quality of life among patients with inflammatory bowel disease. Psychology and Health 2012; 27: 1463 1479

[759] Shaw L, Ehrlich A. Relaxation training as a treatment for chronic pain caused by ulcerative colitis. Pain 1987; 29: $287-293$

[760] Gerbarg PL, Jacob VE, Stevens L et al. The Effect of Breathing, Movement, and Meditation on Psychological and Physical Symptoms and Inflammatory Biomarkers in Inflammatory Bowel Disease: A Randomized Controlled Trial. Inflammatory Bowel Diseases 2015; 21: 2886 2896

[761] Klare P, Nigg J, Nold J et al. The impact of a ten-week physical exercise program on health-related quality of life in patients with inflammatory bowel disease: A prospective randomized controlled trial. Digestion 2015; 91: 239-247

[762] Packer N, Hoffman-Goetz L, Ward G. Does physical activity affect quality of life, disease symptoms and immune measures in patients with inflammatory bowel disease? A systematic review. Journal of Sports Medicine and Physical Fitness 2010; 50: 1-18 
[763] Sharma P, Poojary G, Velez DM et al. Effect of Yoga-Based Intervention in Patients with Inflammatory Bowel Disease. Int J Yoga Therap 2015; 25: 101-112

[764] Cramer H, Schafer M, Schols $M$ et al. Randomised clinical trial: yoga vs written self-care advice for ulcerative colitis. Aliment Pharmacol Ther 2017; 45: $1379-1389$

[765] Schneider A, Streitberger K, Joos S. Acupuncture treatment in gastrointestinal diseases: A systematic review. World J Gastroenterol 2007; 13: $3417-3424$

[766] Joos S, Wildau N, Kohnen R et al. Acupuncture and moxibustion in the treatment of ulcerative colitis: a randomized controlled study. Scand J Gastroenterol 2006; 41: 1056-1063

[767] Ji ], Lu Y, Liu H et al. Acupuncture and moxibustion for inflammatory bowel diseases: a systematic review and meta-analysis of randomized controlled trials. Evid Based Complement Alternat Med 2013; 2013: 158352

[768] Lee DH, Kim JI, Lee MS et al. Moxibustion for ulcerative colitis: a systematic review and meta-analysis. BMC Gastroenterol 2010; 10: 36

[769] Fernandez-Banares F, Hinojosa J, Sanchez-Lombrana JL et al. Randomized clinical trial of Plantago ovata seeds (dietary fiber) as compared with mesalamine in maintaining remission in ulcerative colitis. Spanish Group for the Study of Crohn's Disease and Ulcerative Colitis (GETECCU). Am J Gastroenterol 1999; 94: 427-433

[770] Hanai H, lida T, Takeuchi K et al. Curcumin Maintenance Therapy for Ulcerative Colitis: Randomized, Multicenter, Double-Blind, PlaceboControlled Trial. Clinical Gastroenterology and Hepatology 2006; 4: $1502-1506$

[771] Singla V, Pratap Mouli V, Garg SK et al. Induction with NCB-02 (curcumin) enema for mild-to-moderate distal ulcerative colitis - a randomized, placebo-controlled, pilot study. J Crohns Colitis 2014; 8: 208-214

[772] Lang A, Salomon N, Wu JCY et al. Curcumin in Combination With Mesalamine Induces Remission in Patients With Mild-to-Moderate Ulcerative Colitis in a Randomized Controlled Trial. Clinical Gastroenterology and Hepatology 2015; 13: 1444-1449.e1

[773] Langhorst J, Varnhagen I, Schneider SB et al. Randomised clinical trial: a herbal preparation of myrrh, chamomile and coffee charcoal compared with mesalazine in maintaining remission in ulcerative colitis a double-blind, double-dummy study. Aliment Pharmacol Ther 2013; 38: $490-500$

[774] Albrecht U, Muller V, Schneider B et al. Efficacy and safety of a herbal medicinal product containing myrrh, chamomile and coffee charcoal for the treatment of gastrointestinal disorders: a non-interventional study. BMJ Open Gastroenterol 2014; 1: e000015

[775] Langhorst ], Lauche R, Koch AK. Myrrhe, Kamille und Kaffeekohle in der Therapie von Patienten mit Colitis ulcerosa. Eine retrospektive Kohortenstudie mit 5-Jahres-Follow-up. Zeitschrift für Phytotherapie 2016; 37: $249-253$

[776] Kamali M, Tavakoli H, Khodadoost M et al. Efficacy of the Punica granatum peels aqueous extract for symptom management in ulcerative colitis patients. A randomized, placebo-controlled, clinical trial. Complement Ther Clin Pract 2015; 21: 141 - 146

[777] Gupta I, Parihar A, Malhotra P et al. Effects of gum resin of Boswellia serrata in patients with chronic colitis. Planta Med 2001; 67: 391 - 395
[778] Gupta I, Parihar A, Malhotra P et al. Effects of Boswellia serrata gum resin in patients with ulcerative colitis. Eur J Med Res 1997; 2: 37 - 43

[779] Tang T, Targan SR, Li ZS et al. Randomised clinical trial: herbal extract HMPL-004 in active ulcerative colitis - a double-blind comparison with sustained release mesalazine. Aliment Pharmacol Ther 2011; 33: 194 202

[780] Sandborn W], Targan SR, Byers VS et al. Andrographis paniculata extract (HMPL-004) for active ulcerative colitis. Am J Gastroenterol 2013 108: $90-98$

[781] Ben-Arye E, Goldin E, Wengrower D et al. Wheat grass juice in the treatment of active distal ulcerative colitis: a randomized double-blind placebo-controlled trial. Scand J Gastroenterol 2002; 37: 444-449

[782] Greenfield SM, Green AT, Teare JP et al. A randomized controlled study of evening primrose oil and fish oil in ulcerative colitis. Aliment Pharmacol Ther 1993; 7: 159-166

[783] Langmead L, Feakins RM, Goldthorpe S et al. Randomized, doubleblind, placebo-controlled trial of oral aloe vera gel for active ulcerative colitis. Aliment Pharmacol Ther 2004; 19: 739-747

[784] Rastegarpanah M, Malekzadeh R, Vahedi H et al. A randomized, double blinded, placebo-controlled clinical trial of silymarin in ulcerative colitis. Chin J Integr Med 2015; 21: $902-906$

[785] Tong ZQ, Yang B, Chen BY et al. A multi-center, randomized, singleblind, controlled clinical study on the efficacy of composite sophora colon-soluble capsules in treating ulcerative colitis. Chin J Integr Med 2010; 16: $486-492$

[786] Biedermann L, Mwinyi J, Scharl M et al. Bilberry ingestion improves disease activity in mild to moderate ulcerative colitis - an open pilot study. J Crohns Colitis 2013; 7: 271 - 279

[787] Pullan RD, Rhodes J, Ganesh S et al. Transdermal nicotine for active ulcerative colitis. N Engl J Med 1994; 330: 811 - 815

[788] Sandborn W], Tremaine WJ, Offord KP et al. Transdermal nicotine for mildly to moderately active ulcerative colitis. A randomized, doubleblind, placebo-controlled trial. Ann Intern Med 1997; 126: 364-371

[789] Thomas GA, Rhodes J, Mani V et al. Transdermal nicotine as maintenance therapy for ulcerative colitis. N Engl J Med 1995; 332: 988-992

[790] Thomas GA, Rhodes J, Ragunath K et al. Transdermal nicotine compared with oral prednisolone therapy for active ulcerative colitis. Eur ] Gastroenterol Hepatol 1996; 8: $769-776$

[791] Pagoldh M, Hultgren E, Arnell P et al. Hyperbaric oxygen therapy does not improve the effects of standardized treatment in a severe attack of ulcerative colitis: A prospective randomized study. Scand J Gastroenterol 2013; 48: 1033-1040

[792] Garg SK, Croft AM, Bager P. Helminth therapy (worms) for induction of remission in inflammatory bowel disease. Cochrane Database Syst Rev 2014; 20: CD009400

[793] Summers RW, Elliott DE, Urban JF Jr et al. Trichuris suis therapy for active ulcerative colitis: a randomized controlled trial. Gastroenterology $2005 ; 128: 825-832$

[794] Scholmerich J, Fellermann K, Seibold FW et al. A Randomised, Doubleblind, Placebo-controlled Trial of Trichuris suis ova in Active Crohn's Disease. J Crohns Colitis 2017; 11: $390-399$ 